\title{
Long-term monitoring of the outgassing and composition of comet 67P/Churyumov-Gerasimenko with the Rosetta/MIRO instrument
}

\author{
N. Biver ${ }^{1}$, D. Bockelée-Morvan ${ }^{1}$, M. Hofstadter ${ }^{2}$, E. Lellouch ${ }^{1}$, M. Choukroun ${ }^{2}$, S. Gulkis ${ }^{2, \star}$, J. Crovisier ${ }^{1}$, \\ F. P. Schloerb ${ }^{3}$, L. Rezac ${ }^{4}$, P. von Allmen ${ }^{2}$, S. Lee ${ }^{2}$, C. Leyrat ${ }^{1}$, W. H. Ip ${ }^{5}$, P. Hartogh ${ }^{4}$, P. Encrenaz ${ }^{6}$, \\ G. Beaudin ${ }^{6}$, and the MIRO team
${ }^{1}$ LESIA, Observatoire de Paris, PSL Research University, CNRS, Sorbonne Université, University Paris Diderot, Sorbonne Paris Cité, 5 place Jules Janssen, 92195 Meudon, France e-mail: nicolas.biver@obspm.fr
2 JPL/California Institute of Technology, 4800 Oak Grove Dr., Pasadena, CA 91109, USA
${ }^{3}$ University of Massachusetts, 619 Lederle Graduate Research Tower, Amherst, MA, USA
${ }^{4}$ Max Planck Institut für Sonnensystemforschung, Justus-von-Liebig-Weg 3, 37077 Göttingen, Germany
${ }^{5}$ National Central University, Jhongli, Taoyuan City 32001, Taiwan
${ }^{6}$ LERMA, Observatoire de Paris, PSL Research University, Sorbonne Université, CNRS, 61 avenue de l'Observatoire, 75014 Paris, France

Received 21 December 2018 / Accepted 1 March 2019

\begin{abstract}
We present the analysis of $\approx 100$ molecular maps of the coma of comet $67 \mathrm{P} /$ Churyumov-Gerasimenko that were obtained with the MIRO submillimeter radiotelescope on board the Rosetta spacecraft. From the spectral line mapping of $\mathrm{H}_{2}^{16} \mathrm{O}, \mathrm{H}_{2}^{18} \mathrm{O}, \mathrm{H}_{2}^{17} \mathrm{O}, \mathrm{CH}_{3} \mathrm{OH}$, $\mathrm{NH}_{3}$, and $\mathrm{CO}$ and some fixed nadir pointings, we retrieved the outgassing pattern and total production rates for these species. The analysis covers the period from July 2014, inbound to perihelion, to June 2016, outbound, and heliocentric distances $r_{\mathrm{h}}=1.24-3.65 \mathrm{AU}$. A steep evolution of the outgassing rates with heliocentric distance is observed, typically in $r_{\mathrm{h}}^{-6}$, with significant differences between molecules (e.g. steeper variation for $\mathrm{H}_{2} \mathrm{O}$ post-perihelion than for methanol). As a consequence, the abundances relative to water in the coma vary. The $\mathrm{CH}_{3} \mathrm{OH}$ and $\mathrm{CO}$ abundances increase after perihelion, while the $\mathrm{NH}_{3}$ abundance peaks around perihelion and then decreases. Outgassing patterns have been modeled as 2D Gaussian jets. The width of these jets is maximum around the equinoxes when the bulk of the outgassing is located near the equator. From July 2014 to February 2015, the outgassing is mostly restricted to a narrower jet (full width at half-maximum $\approx 80^{\circ}$ ) originating from high northern latitudes, while around perihelion, most of the gaseous production comes from the southernmost regions $\left(-80 \pm 5^{\circ}\right.$ cometocentric latitude) and forms a $100^{\circ}-130^{\circ}$ (full width at half-maximum) wide fan. We find a peak production of water of $0.8 \times 10^{28} \mathrm{molec} . \mathrm{s}^{-1}, 2.5$ times lower than measured by the ROSINA experiment, and place an upper limit to a $50 \%$ additional production that could come from the sublimation of icy grains. We estimate the total loss of ices during this perihelion passage to be $4.18 \pm 0.18 \times 10^{9} \mathrm{~kg}$. We derive a dust-to-gas ratio in the lost material of $0.7-2.3$ (including all sources of errors) based on the nucleus mass loss of $10.5 \pm 3.4 \times 10^{9} \mathrm{~kg}$ estimated by the RSI experiment. We also obtain an estimate of the $\mathrm{H}_{2}^{18} \mathrm{O} / \mathrm{H}_{2}^{17} \mathrm{O}$ ratio of $5.6 \pm 0.8$.
\end{abstract}

Key words. comets: general - comets: individual: 67P/churyumov-Gerasimenko - radio lines: planetary systems submillimeter: planetary systems

\section{Introduction}

Comets are the most pristine remnants of the formation of the solar system 4.6 billion years ago. Understanding their composition and the sublimation process of their ices as they heat up approaching the Sun was one of the main objectives of the Rosetta mission of the European Space Agency to comet 67P/Churyumov-Gerasimenko (67P). Comet 67P is a Jupiterfamily comet that reached perihelion on 13.1 August 2015 UT at 1.24 AU from the Sun. After a ten-year-long journey in the solar system, the Rosetta spacecraft reached (at a distance to the comet of $<100 \mathrm{~km}$ ) comet $67 \mathrm{P}$ on 6 August 2015, at $3.6 \mathrm{AU}$ from the Sun. It entered into orbit or escorted the nucleus of comet

\footnotetext{
${ }^{\star}$ Research Associate.
}

67P from a 5-1400 km distance until 30.5 September 2016, when Rosetta terminated its operations at the surface of the comet, at 3.83 AU from the Sun (Accomazzo et al. 2016, 2017).

The Microwave Instrument for the Rosetta Orbiter (MIRO) is a submillimeter radiometer equipped with two continuum channels at 188 and $562 \mathrm{GHz}$ and a high spectral resolution $(44 \mathrm{kHz})$ heterodyne spectrometer working in frequency-switching mode (Gulkis et al. 2007). MIRO acquired about two million spectra during its operation at the comet. Four molecules were targeted through their rotational lines in the $548-579 \mathrm{GHz}$ range: $\mathrm{H}_{2} \mathrm{O}, \mathrm{CH}_{3} \mathrm{OH}, \mathrm{CO}$, and $\mathrm{NH}_{3}$. In addition to $\mathrm{H}_{2}^{16} \mathrm{O}, \mathrm{H}_{2}^{18} \mathrm{O}$ and $\mathrm{H}_{2}^{17} \mathrm{O}$ were observed to handle the frequently occurring saturation of the main isotopic species. For methanol, three lines were observed to measure the rotational temperature. 
First results on the MIRO observations of water in early June and August 2014 were published by Gulkis et al. (2015). Biver et al. (2015) analyzed a map of the water lines obtained on 7 September 2014, at 3.41 AU from the Sun. Lee et al. (2015) presented a study of the diurnal variations of the water lines observed in August 2014 at $r_{\mathrm{h}}=3.60-3.52$ AU. Marshall et al. (2017) provided an analysis of the water production rate evolution from August 2014 until April 2016, covering an $r_{\mathrm{h}}$ range of 3.62-1.24 AU, based on MIRO nadir observations that sampled the local outgassing.

We analyze here dedicated mapping observations acquired by slewing the Rosetta spacecraft. These observations were spread over the two years of the Rosetta mission. They provide information along the three dimensions, including the $(X, Y)$ mapping coordinates and along the line of sight $(Z)$. The $Z$ axis data are provided by the spectral line resolved velocity information, obtained with the high-resolution spectrometer.

Hundreds of raster maps were acquired with sampling and scanning speed adapted to different objectives: (1) mapping the nucleus and near coma (e.g., Biver et al. 2015), (2) mapping the coma with a scanning speed adapted to the MIRO acquisition duration, or (3) faster mapping of the coma appropriate to the high-resolution channel of the Visual IR Thermal Imaging Spectrometer (VIRTIS-H). In addition, sequences sampling a few positions in the coma (coarse maps) were performed. In Table A.1 we list the MIRO maps and coarse maps considered in this paper. A full analysis has been performed for three quarters of these maps.

Section 2 describes the observations and the data reduction and calibration. Section 3 describes the model we used to interpret the data and how the expansion velocity and gas temperature were obtained. Section 4 is a step-by-step description of our map analysis. Section 5 presents a discussion of the errors that result from modeling approximations. Section 6 discusses some of the individual maps. In Sect. 7 we make use of nadir observations to derive longitudinal averages of the production rates as a function of the cometocentric latitude. In Sect. 8 we investigate the possible contribution of distributed sources of water in the coma. Section 9 presents a determination of the $\mathrm{H}_{2}^{18} \mathrm{O} / \mathrm{H}_{2}^{17} \mathrm{O}$ ratio. Section 10 presents the long-term evolution of the outgassing rates and relative production rate ratios. We also provide the cumulative mass loss of volatiles by the comet. We compare the results with measurements from other instruments. Section 11 concludes with a summary of the results. The processed data (maps and plots) are contained in the appendices.

\section{Observations}

\subsection{Mapping of submillimeter lines}

The MIRO instrument operated as a single-beam submillimeter radiotelescope mounted rigidly on the Rosetta spacecraft. The pointing axis was aligned with the $+Z$ axis of the spacecraft, as were most of the other remote-sensing instruments (Koschny et al. 2007). During the entire Rosetta observation period, MIRO operated continuously, mostly in "CTS/dual-continuum" mode (Gulkis et al. 2015). In this mode, frequency-switched spectra at wavelengths $\sim 0.53 \mathrm{~mm}$ were acquired every $30 \mathrm{~s}$, and continuum measurements at 0.53 and $1.6 \mathrm{~mm}$ wavelengths were obtained every $50 \mathrm{~ms}$. Spectra were obtained with a chirp transform spectrometer (CTS). In some periods, the data rate available to transmit data to the Earth was limited. The data volume was then reduced by averaging spectra or continuum measurements on longer timescales and eventually smoothing the spectra to reduce
Table 1. Lines observed by MIRO.

\begin{tabular}{lccr}
\hline \hline Molecule & Transition & $\begin{array}{c}\text { Frequency } \\
(\mathrm{MHz})\end{array}$ & $\begin{array}{r}\text { Upper level } \\
\text { energy (K) }\end{array}$ \\
\hline $\mathrm{H}_{2}^{16} \mathrm{O}$ & $1_{1,0}-1_{0,1}$ & 556936.002 & 60.96 \\
$\mathrm{H}_{2}^{18} \mathrm{O}$ & $1_{1,0}-1_{0,1}$ & 547676.440 & 60.46 \\
$\mathrm{H}_{2}^{17} \mathrm{O}$ & $1_{1,0}-1_{0,1}$ & 552021.305 & 60.70 \\
$\mathrm{CH}_{3} \mathrm{OH}$ & $3_{-2}-2_{-1} \mathrm{E}$ & 568566.082 & 39.83 \\
$\mathrm{CH}_{3} \mathrm{OH}$ & $8_{+1}-7_{0} \mathrm{E}$ & 553146.224 & 104.62 \\
$\mathrm{CH}_{3} \mathrm{OH}$ & $12_{-1}-11_{-1} \mathrm{E}$ & 579150.985 & 186.43 \\
$\mathrm{NH}_{3}$ & $1_{0,0}-0_{0,1}$ & 572498.371 & 27.48 \\
$\mathrm{CO}$ & $5-4$ & 576267.933 & 82.97 \\
\hline
\end{tabular}

the number of channels. The beam width at $560 \mathrm{GHz}$ is $7.5^{\prime}$. As the instrument has a single beam, it was necessary to slew the whole spacecraft over a larger field of view in order to obtain spatial information. The slew rate minimized smearing during individual integrations times. Values from 15 to $30^{\prime}$ per minute were used for MIRO spectral maps.

Table 1 lists the lines of $\mathrm{H}_{2}^{16} \mathrm{O}, \mathrm{H}_{2}^{18} \mathrm{O}, \mathrm{H}_{2}^{17} \mathrm{O}, \mathrm{CH}_{3} \mathrm{OH}, \mathrm{NH}_{3}$, and $\mathrm{CO}$ that are observed in MIRO spectra. Although carbon monoxide is a relatively abundant species, observing it with MIRO was much more difficult than were observations for the other molecules. It only showed up well in periods of high activity because the rotational lines of this molecule are weak since $\mathrm{CO}$ has a small dipole moment, and because the MIRO spectrometer could only observe the CO $J(5-4)$ line, a transition between relatively high rotational energy levels.

Table A.1 provides the list of the maps we analyze here, together with their characteristics. Their sizes range from $2^{\circ}$ to $30^{\circ}$ ( $\sim 4$ to $45 \mathrm{~km}$ considering the distance to the comet). They were typically acquired in $4 \mathrm{~h}$, which corresponds to about onethird of the rotation period of the comet of $\approx 12 \mathrm{~h}$. Figure 1 shows examples of scanning schemes with measured line intensities. The maps shown in Appendix B (Figs. B.1-B.14 for $\mathrm{H}_{2}^{18} \mathrm{O}$ and Fig. B.15 for $\mathrm{H}_{2}^{16} \mathrm{O}$ ) were interpolated for better visualization. For the analysis, however, we used the spectra at their mean locations.

In addition, discrete point-by-point spatial maps were also obtained without a continuous slewing, but still regularly sampling the coma. These were made in periods of low comet activity.

The geometric data provided with the observations were used to compute the coordinates of each observation and the pointing offset relative to the nucleus in the J2000.0 RA/Dec frame. From the RA/Dec coordinates of the spin axis of the comet (Jorda et al. 2016) and the coordinates of the Sun from 67P provided by the JPL Horizons tool (Giorgini et al. 2018), we computed the projected position angle (PA) in the plane of the sky and the aspect angles (Rosetta-comet-north pole and Rosetta-cometSun) for the north pole and the Sun directions. Because all maps were made by scanning along the Sun-comet projected vector (Fig. 1), we rotated all maps so that the Sun direction was either to the left $\left(\mathrm{PA}=90^{\circ}\right)$ or right $\left(\mathrm{PA}=270^{\circ}\right)$. All pointing offsets were further converted into kilometers relative to the center of $67 \mathrm{P}$.

\subsection{Reduction and calibration of the MIRO data}

The MIRO $f_{0}=562 \mathrm{GHz}$ submillimeter local oscillator frequency is switched by $\pm \Delta f=5 \mathrm{MHz}$ every $5 \mathrm{~s}$. The $30 \mathrm{~s}$ CTS 

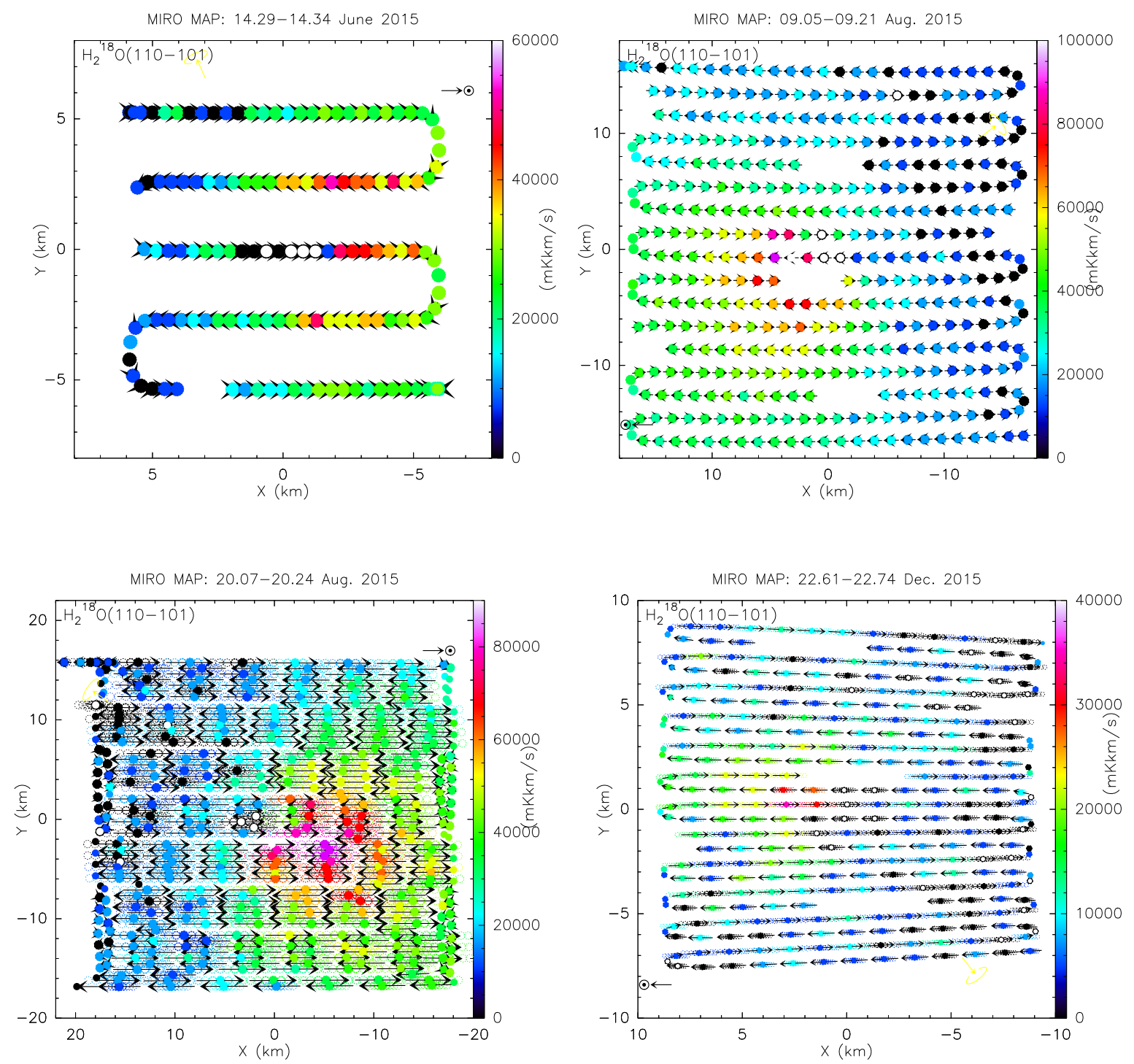

Fig. 1. Examples of schemes used to scan the coma of $67 \mathrm{P}$ with Rosetta/MIRO. Each circle has the projected size of the MIRO beam width $\left(7.5^{\prime}\right)$ at the distance of the comet and is projected at the pointed position at the center of the $30 \mathrm{~s}$ integration. The scales of the $X$-and $Y$-axes are the distance from comet center. Arrows show the scanning direction and its motion during $30 \mathrm{~s}$. The color provides the $\mathrm{H}_{2}^{18} \mathrm{O}$ integrated line intensity according to the scale given on the right. The Sun-projected direction is indicated. Blanks along the sampling correspond to data gaps during calibration (once every $30 \mathrm{~min}$ ).

spectra consist of the average of three pairs of $f^{+}\left(=f_{0}+\right.$ $\Delta f)-f^{-}\left(=f_{0}-\Delta f\right)$ frequency-switched spectra (Gulkis et al. 2015). A calibration was performed to determine the gain of the receiver every $\sim 30$ min by observing a hot load, a cold load, and the sky in sequence, each for $30 \mathrm{~s}$. A motor-driven flip mirror mounted on the optical bench provides the switching motion. These calibration data were obtained separately for each local oscillator frequency $f^{+}$and $f^{-}$. We used spline fitting over $6 \mathrm{~h}$ (continuum mode) to $36 \mathrm{~h}$ (spectral mode) to calibrate continuum measurements (separately for each frequency) and spectral data in each sub-band of the CTS. Further information on the calibration may be found in Schloerb et al. (2015). We list the additional corrections that were applied below.

1. Beam efficiency correction $\eta_{\mathrm{b}}=0.94$ to convert antenna temperatures $T_{\mathrm{A}}^{*}$ into main beam brightness temperatures $T_{\mathrm{mb}}=T_{\mathrm{A}}^{*} / \eta_{\mathrm{b}}$. This value was suggested by Schloerb et al. (2015) based on measurements obtained before flight. The beam was described as a main beam of Gaussian profile with a full width at half-maximum $F W H M\left[{ }^{\prime \prime}\right]=$ $456 \times(562.8 / v(\mathrm{GHz}))$, and an additional error beam containing $3 \%$ of the signal that was ten times broader. This is within $1 \%$ of the values determined from the MIRO flight model on ground with an assumed $1 / v$ frequency dependency. This beam efficiency correction was applied to spectral and continuum measurements.

2. Side-band gain correction $\mathrm{gc}_{1}=1.12$ for spectra obtained in the lower side band $\left(f<f_{0}\right.$, i.e., the water lines and one line of $\mathrm{CH}_{3} \mathrm{OH}$ ) and $\mathrm{gc}_{\mathrm{u}}=0.90$ for the upper side band (i.e., $\mathrm{NH}_{3}$, $\mathrm{CO}$, and two $\mathrm{CH}_{3} \mathrm{OH}$ lines). In previous publications and archived data, the side-band gain ratio was assumed to be 1.0. It was not precisely measured before flight, but deviations by more than $10 \%$ were not excluded. When the saturated part (channels around $-0.8 \mathrm{~km} \mathrm{~s}^{-1}$ ) of the $\mathrm{H}_{2}^{16} \mathrm{O}$ line is considered, the intensity $T_{\text {em }}$, measured against the blank sky, is expected to be equal to the intensity $T_{\mathrm{bg}}-T_{\mathrm{abs}}$ measured in absorption in front of the thermal radiation of the nucleus (see, e.g., Fig. 2), but this is not observed. Whereas the 

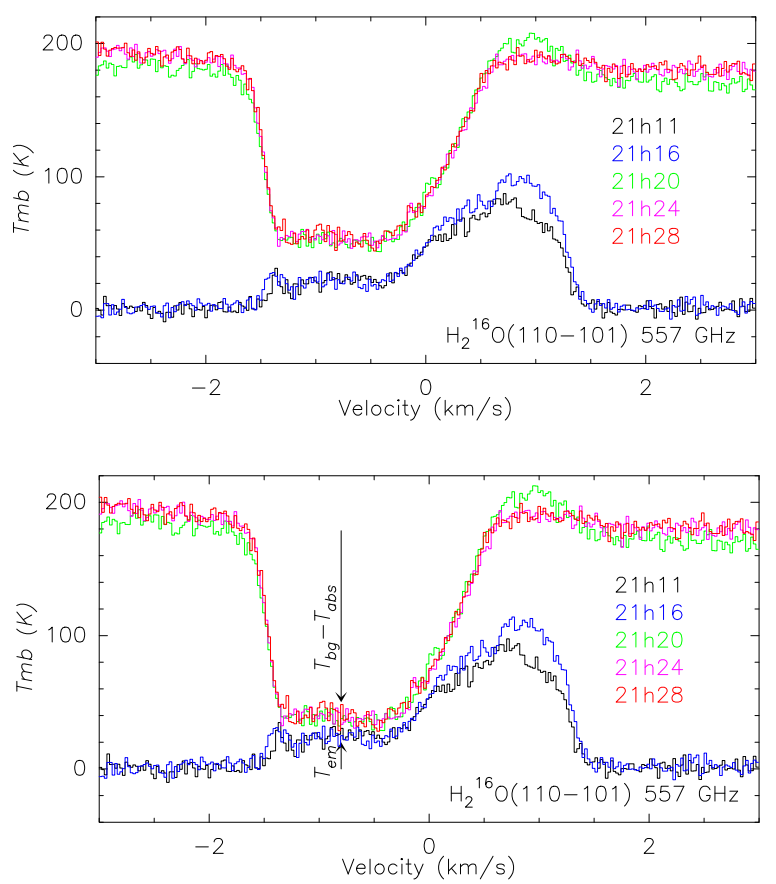

Fig. 2. Spectra of $\mathrm{H}_{2}^{16} \mathrm{O}$ obtained with MIRO on 24 September 2015. The five spectra are $4 \mathrm{~min}$ averages that were obtained during a sequence where the line of sight moved from limb sounding to nadir viewing, and the background changed from 0 to $200 \mathrm{~K}$. The line is saturated between -1.3 and $-0.4 \mathrm{~km} \mathrm{~s}^{-1}$, where its intensity corresponds to the gas temperature near the spacecraft. Top: before applying the side-band gain correction. Bottom: after applying this correction.

continuum background temperature

$T_{\mathrm{bg}}=0.5\left(g_{l} T_{\mathrm{bg}}+g_{u} T_{\mathrm{bg}}\right)$

originates from the two side bands and does not depend on the the side-band gain ratio $g_{\mathrm{u}} / g_{1}$ because $g_{\mathrm{u}}+g_{1}=2.0$, the line intensity does depend on the side-band ratio, for instance, $T_{\mathrm{em}}$ (measured) $=g_{\mathrm{l}} T_{\mathrm{em}}$ for a line in lower side band. Based on a large set of saturated $\mathrm{H}_{2}^{16} \mathrm{O}$ lines around perihelion, we determine the side-band gain $g_{1}$ so that

$T_{\mathrm{em}}$ (measured) $/ g_{1}=T_{\mathrm{bg}}-T_{\mathrm{abs}}($ measured $) / g_{1}$

is satisfied. We found $g_{1}=0.89\left(\mathrm{gc}_{1}=1 / g_{1}=1.12\right)$ and $g_{\mathrm{u}}=1.11\left(\mathrm{gc}_{\mathrm{u}}=1 / g_{\mathrm{u}}=0.90\right)$. We used the same correction for all MIRO bands. After applying the side-band correction, saturated $\mathrm{H}_{2}^{16} \mathrm{O}$ profiles in limb and nadir sounding present consistent intensities at negative velocities, as illustrated in Fig. 3. We note that the $\mathrm{CH}_{3} \mathrm{OH}\left(8_{+1}-7_{0} E\right)$ line, observed in lower side band, is always weaker than expected in comparison to the $\mathrm{CH}_{3} \mathrm{OH}\left(3_{-2}-2_{-1} E\right)$ and $\mathrm{CH}_{3} \mathrm{OH}\left(12_{-1}-11_{-1} E\right)$ lines that are observed in upper side band. After applying the side-band correction, the discrepancy is reduced, although the production rates derived from the different methanol lines suggest that a larger correction would be needed.

The $30 \mathrm{~s}$ average continuum values were added as ancillary data to each spectrum. They were used to analyze the absorption spectra that were obtained in nadir viewing (Sect. 7).

\section{Modeling approach}

Before describing the step-by-step process we employed to derive the outgassing pattern and total production for each

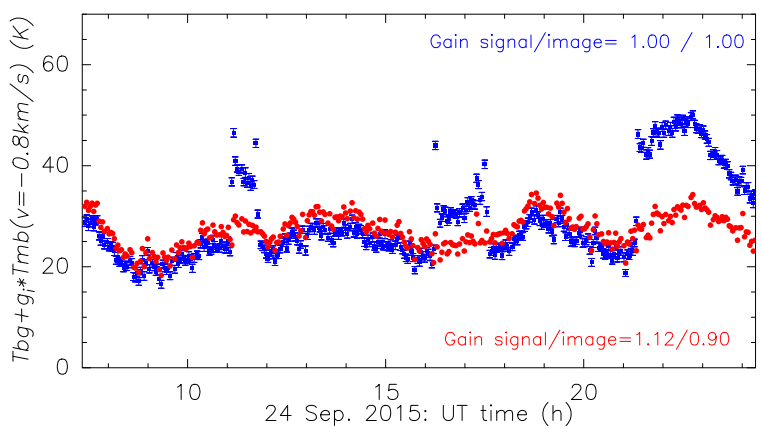

Fig. 3. Intensity measured at $-0.8 \mathrm{~km} \mathrm{~s}^{-1}$ on saturated $\mathrm{H}_{2}^{16} \mathrm{O}$ spectra obtained on 24 September 2015 between 7.5 and 24 h UT while scanning across the nucleus (see the spectrum examples in Fig. 2). The blue and red dots show the value before and after gain correction, respectively. The jumps before correction correspond to times when MIRO pointed on the nucleus. Error bars are only plotted for the uncorrected data.

molecule based on the maps, we describe below the geometrical approach of the problem, the excitation and radiative transfer codes we used to compute line profiles and intensities. We also describe how we derived two key parameters for these codes, the gas expansion velocity and temperature.

\subsection{Definition of parameters and model}

In Table 2 we list all the variables introduced in this paper with their units and definition. We have modeled the comet outgassing as the superposition of an isotropic component and a jet-like component with a Gaussian distribution,

$q(\alpha)=q_{\mathrm{I}}+q_{\mathrm{J}} \exp \left(-\frac{\alpha^{2}}{\sigma_{J}^{2}}\right)$,

with

$\sigma_{\mathrm{J}}=\frac{\theta_{\mathrm{J}}}{2 \cdot \ln 2}$

Here $q_{\mathrm{I}}$ is the isotropic production rate per solid angle, $q_{\mathrm{J}}$ is the peak production rate per solid angle in the jet, and $\theta_{\mathrm{J}}$ is the FWHM of this Gaussian jet.

Figure 4 illustrates the main geometrical parameters of the jet, represented in this figure as a simple cone.

\subsection{Excitation and outgassing pattern model}

We used the excitation and radiative transfer codes described in previous studies (Biver et al. 2015, 2012; Zakharov et al. 2007), which take into account collisions with neutrals and electrons, radiative decay, and infrared pumping of the rotational levels. The line opacity was taken into account for the excitation using the escape probability formalism. The rotational populations were derived for each distance $r$ to the nucleus with a time evolution linked to the distance through the radial expansion $\left(r(t)=\int v_{\exp }(r) \mathrm{d} t\right)$. Collisional excitation depends on the gas temperature $T(r)$ as determined hereafter. The computations started at a radius $r=2 \mathrm{~km}$, and all parameters (relative populations, gas temperature, and expansion velocity) were assumed to have the same value for $r<2 \mathrm{~km}$ as for $r=2 \mathrm{~km}$. For collisions with water molecules, we used total cross sections of $5 \times 10^{-14} \mathrm{~cm}^{2}$ for methanol and water, and $2 \times 10^{-14} \mathrm{~cm}^{2}$ for ammonia and $\mathrm{CO}$. 
Table 2. List of variables.

\begin{tabular}{|c|c|c|c|}
\hline Symbol & Variant & Unit & Description \\
\hline \multicolumn{4}{|c|}{ Coordinate systems (with origin at the center of the comet nucleus) } \\
\hline \multirow{3}{*}{\multicolumn{2}{|c|}{$\begin{array}{l}(r, \theta, \phi) \\
(\rho, \theta, z) \\
(X, Y, Z)\end{array}$}} & $\left(\mathrm{m},{ }^{\circ},{ }^{\circ}\right)$ & Spherical coordinates (radius, azimuth, colatitude) \\
\hline & & $\left(\mathrm{m},{ }^{\circ}, \mathrm{m}\right)$ & Cylindrical coordinates \\
\hline & & $(\mathrm{km}, \mathrm{km}, \mathrm{km})$ & Cartesian coordinates as defined in Koschny et al. (2007) \\
\hline \multicolumn{2}{|l|}{$r_{\mathrm{h}}$} & (AU) & Heliocentric distance \\
\hline \multicolumn{2}{|l|}{$\Delta$} & $(\mathrm{km})$ & Distance of Rosetta to the comet \\
\hline \multicolumn{2}{|l|}{$r$} & $(\mathrm{~m}$ or $\mathrm{km})$ & Distance to the center of the nucleus \\
\hline \multicolumn{2}{|l|}{$\rho$} & $(\mathrm{m}$ or $\mathrm{km})$ & Distance to the center of the nucleus in the $X, Y$ mapping plane \\
\hline$T, T_{\text {rot }}$ & $T(\mathrm{r}), T_{\text {rot }}(\rho)$ & $(\mathrm{K})$ & Gas temperature, rotational temperature; as a function of $r$ or $\rho$ \\
\hline \multirow{2}{*}{$v_{\exp }$} & $v_{\mathrm{exp}}(r)$ & $\left(\mathrm{km} \mathrm{s}^{-1}\right)$ & Mean gas expansion velocity; depending on radial distance $r$ \\
\hline & $v_{\mathrm{i}}$ & $\left(\mathrm{km} \mathrm{s}^{-1}\right)$ & Doppler velocity relative to the nucleus; of a spectral channel i \\
\hline \multicolumn{2}{|l|}{$\Delta v$} & $\left(\mathrm{~km} \mathrm{~s}^{-1}\right)$ & Mean Doppler shift of a whole line $\int\left(T_{\mathrm{mb}}(v) v \mathrm{~d} v\right) / \int\left(T_{\mathrm{mb}}(v) \mathrm{d} v\right)$ \\
\hline \multicolumn{2}{|l|}{$\alpha^{(a)}$} & ( ${ }^{\circ}$ or radian) & Angle between the jet axis and considered direction \\
\hline \multirow{2}{*}{$\begin{array}{l}\mathrm{PA}^{(a)} \\
\phi^{(a)}\end{array}$} & $\mathrm{PA}_{\text {jet }}, \mathrm{PA}_{\text {pole }}$ & $\left({ }^{\circ}\right)$ & Position angle counted from the $+Y$ axis in the $(X, Y)$ map plane \\
\hline & $\phi_{\mathrm{J}}, \phi_{i}, \phi_{\text {pole }}$ & $\left({ }^{\circ}\right)$ & Colatitude or polar angle, counted from the $-Z$ axis \\
\hline \multirow[t]{4}{*}{$\theta_{J}^{(a)}$} & $\sigma_{\mathrm{J}}=\frac{\theta_{\mathrm{J}}}{2 \sqrt{(\ln 2)}}$ & $\left({ }^{\circ}\right)$ & FWHM of the (Gaussian) jet \\
\hline & $\theta_{\text {proj }}$ & $\left({ }^{\circ}\right)$ & FWHM of the jet in the $X, Y$ plane \\
\hline & $\theta_{\phi}$ & $\left({ }^{\circ}\right)$ & FWHM of the jet in colatitude: (jet, $Z$ ) plane) \\
\hline & $\theta_{\mathrm{az}}$ & $\left({ }^{\circ}\right)$ & FWHM of the jet in the plane perpendicular to (jet, $Z)\left(\theta_{\mathrm{az}}<\theta_{\text {proj }}\right)$ \\
\hline \multirow[t]{3}{*}{$A$} & $A(\mathrm{PA}, \rho)$ & $\left(\mathrm{mK} \mathrm{km} \mathrm{s}^{-1}\right)$ & Line area (integrated intensity); at a given point $(\mathrm{PA}, \rho)$ of a map \\
\hline & $A_{0}, A_{\mathrm{p}}$ & $\left(\mathrm{mK} \mathrm{km} \mathrm{s}^{-1}\right)$ & Line area values for a fit: background $\left(A_{0}\right)$ and Peak value $\left(A_{\mathrm{p}}\right)$ \\
\hline & $A_{\mathrm{I}}, A_{\mathrm{J}}$ & $\left(\mathrm{mK} \mathrm{km} \mathrm{s}^{-1}\right)$ & Line area values for a fit: isotropic and jet contribution \\
\hline \multicolumn{2}{|l|}{$T_{\mathrm{A}}^{*}, T_{\mathrm{mb}}$} & $(\mathrm{K})$ & Line intensity scale: antenna or main beam temperature \\
\hline \multicolumn{2}{|l|}{$\eta_{\mathrm{B}}$} & - & Main beam efficiency: $T_{\mathrm{A}}^{*}=\eta_{\mathrm{B}} T_{\mathrm{mb}}$ \\
\hline \multirow[t]{2}{*}{$T_{\mathrm{p}}$} & $T_{\mathrm{em}}, T_{\mathrm{abs}}$ & $(\mathrm{K})$ & Peak intensity of a line (Rayleigh-Jeans equivalent temperature) \\
\hline & $T_{\mathrm{p}(\mathrm{v})}, T_{\mathrm{p}(\rho)}$ & $(\mathrm{K})$ & Peak intensity of a line for a given velocity channel, at a given projected distance \\
\hline \multicolumn{2}{|l|}{$T_{\mathrm{bg}}$} & $(\mathrm{K})$ & Background nucleus (or dust) (Rayleigh-Jeans) temperature (continuum) \\
\hline \multicolumn{2}{|l|}{$\Omega_{\text {jet }}$} & $(\mathrm{sr})$ & Jet solid angle $=2 \pi \int \exp \left(-\frac{\alpha^{2}}{\sigma^{2}}\right) \sin (\alpha) \mathrm{d} \alpha$ \\
\hline \multirow[t]{2}{*}{$q$} & $q(\alpha)$ & $\left(\right.$ molec. $\left.\mathrm{s}^{-1} \mathrm{sr}^{-1}\right)$ & Production rate per solid angle; as a function of angle $\alpha$ \\
\hline & $q_{\mathrm{i}}(\phi), q_{\text {nadir }}$ & (molec. $\mathrm{s}^{-1} \mathrm{sr}^{-1}$ ) & Production rate per solid angle as a function of $\phi$ (derived from channel i) or nadir value $(\phi=0)$ \\
\hline$q_{\mathrm{I}}$ & $q_{\mathrm{I}, \phi}, q_{\mathrm{I}, \mathrm{az}}$ & (molec. $\left.\mathrm{s}^{-1} \mathrm{sr}^{-1}\right)$ & Production rate per solid angle for isotropic contribution; derived along the $\phi$ angle or perpendicularly (az) \\
\hline \multirow{3}{*}{$\begin{array}{l}q_{\mathrm{J}} \\
Q, Q_{\mathrm{total}}\end{array}$} & $q_{\mathrm{J}, \phi}, q_{\mathrm{J}, \mathrm{az}}$ & (molec. $\left.\mathrm{s}^{-1} \mathrm{sr}^{-1}\right)$ & Production rate per solid angle in the jet: peak value; derived along the $\phi$ angle or perpendicularly (az) \\
\hline & $Q(\rho), Q(r)$ & $\left(\right.$ molec. $\left.\mathrm{s}^{-1}\right)$ & Total production rate (for a given molecule); locally as a function of $\rho$ or $r$ \\
\hline & $Q\left(r_{\mathrm{h}}\right)$ & $\left(\right.$ molec. $\left.\mathrm{s}^{-1}\right)$ & Heliocentric variation in total production rate of a given molecule \\
\hline \multicolumn{2}{|l|}{$Q_{\mathrm{I}}, Q_{\mathrm{J}}$} & $\left(\right.$ molec. $\left.\mathrm{s}^{-1}\right)$ & Production rate in the isotropic, jet component: $Q=Q_{\mathrm{I}}+Q_{\mathrm{J}}$ \\
\hline
\end{tabular}

Notes. ${ }^{(a)}$ See Fig. 4.

After the rotational populations were computed, radiative transfer was solved by performing a numerical integration (with adaptive steps) along the line of sight and over the main beam solid angle. Optical depth effects are thus fully considered for each transition. The pointing offset relative to the direction of the center of the nucleus was taken into account as well as the contribution of the continuum emission from the nucleus (and hidden background gas), when parts of the nucleus were in the beam. The observed continuum background was adjusted to the observed value.

In the radiative transfer model, a jet-like outgassing pattern was considered by restricting the outgassing in a region defined by a range in $\theta$ and $\phi$ angles: $q=q_{\mathrm{J}}$ if $\phi_{2}<\phi<\phi_{1}$ and $0<\theta<\theta_{m}$, where $\theta$ (azimuth) and $\phi$ (colatitude) are spherical coordinates centered on the comet. The corresponding solid angle is $\Omega=2 \theta_{m}\left(\cos \left(\phi_{1}\right)-\cos \left(\phi_{2}\right)\right)$, with $\theta_{m}<\pi$. When we fit the data, the $\theta_{m}, \phi_{1}, \phi_{2}$ parameters were adjusted so that this synthetic jet was close to the observed one: same direction, similar jet widths $\theta_{\phi}$ and $\theta_{\mathrm{az}}$ in the plane of the sky and along the line of sight, and same solid angle $\Omega_{\text {jet }}$. The populations of the rotational levels only depend on the distance to the comet center $r$.

\subsection{Determination of the gas expansion velocity}

A first estimate of the gas expansion velocity was obtained from line profiles seen in absorption against the thermal emission of the nucleus in nadir pointing. Examples of these lines are provided in Fig. E.1. The absorption peak seen in the lines is at the Doppler velocity of the bulk of the molecules moving toward the spacecraft, which is the terminal expansion velocity $v_{\exp }(r=\infty)$. Close to the surface, the gas is accelerated and $v_{\exp }(r)$ increases quickly. Rotational lines from high-energy levels $\left(\mathrm{CO}(5-4)\right.$ and $\left.\mathrm{CH}_{3} \mathrm{OH}\left(12_{-1}-11_{-1} E\right)\right)$ are formed closer to the surface (where the gas temperature is higher) and tend to show lower Doppler velocity values at the absorption peak. To simplify the calculation and limit the number of free parameters, we assumed a constant $v_{\text {exp }}$, especially as acceleration only affected the very first kilometers from the surface. A moderate variation of about $+10 \%$ between $r=3$ and $10 \mathrm{~km}$ was taken into account when necessary for the high activity phase around perihelion, however.

The expansion velocity along the line of sight can be different from the gas velocity in the plane of the sky or along the jet 


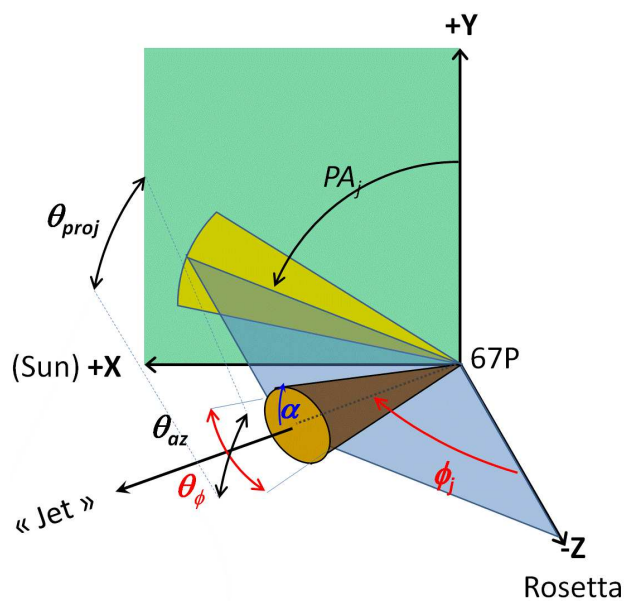

Fig. 4. Geometric parameters defining the jet and its appearance on the plane of the sky, defined by the Rosetta $X$ - and $Y$-axis. The $+Y$ direction is closest to the north pole and the Sun is either in the $+X$ or $-X$ direction $\left(\mathrm{PA}=90^{\circ}\right.$ or $\left.270^{\circ}\right)$.

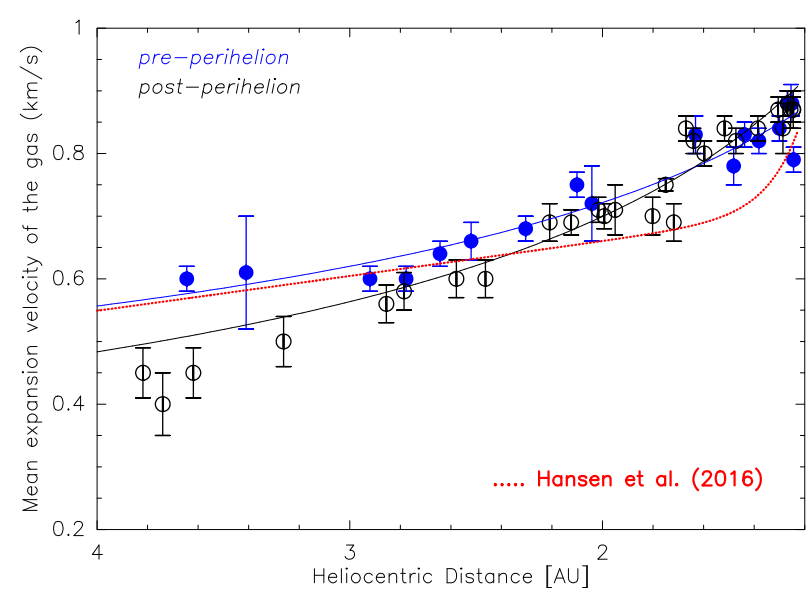

Fig. 5. Mean gas expansion velocity measured for the jet as given in Table A.2. These values were used to invert the lines profiles and compute production rates for all molecules. Filled blue dots show preperihelion data and empty black symbols represent post-perihelion observations. The fitted power laws are $v_{\exp }=0.94 r_{\mathrm{h}}^{-0.38} \mathrm{~km} \mathrm{~s}^{-1}$ preperihelion and $v_{\exp }=1.01 r_{\mathrm{h}}^{-0.53} \mathrm{~km} \mathrm{~s}^{-1}$ post-perihelion, with uncertainties of $0.01 \mathrm{~km} \mathrm{~s}^{-1}$ and 0.03 on the power-law index. For comparison we plot the adopted (water) expansion velocity profile of Hansen et al. (2016) as a red dotted line.

axis. We have little constraint on the velocity perpendicular to the line of sight. Some observations (e.g., when the line of sight in nadir pointing was close to the jet axis) suggest little variation within the jet. $v_{\exp }$ might be $10 \%$ higher in the densest part of the jet, but this does not significantly affect the retrieved production rates. When we inverted the line profiles, we iteratively adjusted $v_{\text {exp }}$ to achieve reasonable agreement between the simulated line profile and the feet of the lines, which correspond to molecules moving close to the line of sight (see, e.g., top of Figs. 10 and 11). The values we used are provided in Table A.2 and plotted in Fig. 5. For comparison we show the velocity profile adopted by Hansen et al. (2016) and Läuter et al. (2019) for water. We note that we used the same expansion velocity for all molecules as we do not see evidence of difference in velocity (e.g., due to their mass) between molecules, but the lower expansion velocity measured post-perihelion beyond $2 \mathrm{AU}$ could be due to mass load by $\mathrm{CO}_{2}$, which is more abundant in the coma (Läuter et al. 2019).

\subsection{Gas temperature profile $T(r)$}

We have estimated the kinetic temperature profile $T(r)$ as a function of the radial distance $r$ from the center of the nucleus using constraints from the rotational temperature of methanol and the peak brightness temperature of the saturated $\mathrm{H}_{2}^{16} \mathrm{O}$ line.

\subsubsection{Rotational temperature of the methanol lines}

We used the two rotational lines of methanol observed in the same side band: $\mathrm{CH}_{3} \mathrm{OH}\left(3_{-2}-2_{-1} E\right)$ and $\mathrm{CH}_{3} \mathrm{OH}\left(12_{-1}-11_{-1} E\right)$. The third line, $\mathrm{CH}_{3} \mathrm{OH}\left(8_{+1}-7_{0} E\right)$, suffers from calibration issues (see Sect. 2.2), and was not used for this study. Because the sampled columns are the same, the rotational temperature $T_{\text {rot }}\left(\mathrm{CH}_{3} \mathrm{OH}\right)$ can be readily derived from the intensity ratio of the two lines using well-known spectroscopic parameters: the energy of the levels, their statistical weights, and Einstein coefficients. In the inner coma, which is dominated by collisions, we expect methanol to be at local thermal equilibrium (LTE). For optically thin lines, the rotational temperature should then correspond to the gas temperature. Based on radiative transfer calculations, we verified that these conditions are reached for all the $T_{\text {rot }}\left(\mathrm{CH}_{3} \mathrm{OH}\right)$ values.

Even at the activity maximum of the comet, the signalto-noise ratio (S/N) of the $\mathrm{CH}_{3} \mathrm{OH}\left(12_{-1}-11_{-1} E\right)$ line was still insufficient to obtain a full map of $T_{\text {rot }}\left(\mathrm{CH}_{3} \mathrm{OH}\right)$. However, by averaging the signals over an angular sector and a range of radial offsets from the nucleus, we were able to derive the radial profile of $T_{\text {rot }}\left(\mathrm{CH}_{3} \mathrm{OH}\right)$ as a function of the offset $\rho$. For additional spatial information, we divided the lines into five velocity bins corresponding to different velocity directions and distances, providing us with measurements of $T_{\text {rot }}\left(\mathrm{CH}_{3} \mathrm{OH}\right)$ at different radii $r$ $\left(r=\rho / \cos (\psi)\right.$ with $\sin (\psi)=v / v_{\text {exp }}$, see Fig. 7$)$.

\subsubsection{Temperature from the saturated water line}

Simulation of line profiles at radial offset $\rho$ shows that when the $\mathrm{H}_{2}^{16} \mathrm{O}$ line is saturated, the main beam brightness temperature $T_{\mathrm{p}}\left(\mathrm{H}_{2}^{16} \mathrm{O}\right)$ at velocity channels $v_{i}=+0.5 \pm 0.2 \mathrm{~km} \mathrm{~s}^{-1}$ corresponds to the peak intensity of the line within $1 \mathrm{~K}$, and at first approximation probes the gas temperature at the distance $\rho$. Simulations were made for the range of production rates and radial offsets appropriate to the MIRO observations we analyze here. In addition, in nadir and limb viewing, the main beam brightness temperature measured in the velocity channel at $v_{i}=-v_{\exp }$ should correspond to the gas temperature close to the spacecraft $T(\Delta)$.

Collisions with electrons may affect the temperature retrieval when collisions between neutrals are low, that is, when the water production rate is low. For high production rates $\left(q_{\mathrm{H}_{2}^{16} \mathrm{O}}>3 \times 10^{26}\right.$ molec. $\left.\mathrm{s}^{-1} \mathrm{sr}^{-1}\right)$, this is only significant at distances $>100 \mathrm{~km}$. We found that for lower production rates, water excitation is less affected by electron collisions because of the lower electron densities, and $T\left(\mathrm{H}_{2}^{16} \mathrm{O}\right)$ can be only up to $5 \mathrm{~K}$ higher than the gas temperature. The effect of collisions with electrons could have been observed in the maps by an increase in $T_{\mathrm{p}}\left(\mathrm{H}_{2}^{16} \mathrm{O}\right)$ at some distance, and also by an increase in brightness temperature at the channels around $v_{i}=-v_{\exp }$ (sampling the spacecraft region). However, the observations show no evidence for such an enhancement, suggesting a relatively low electron density that is consistent with the electron density scaling factor $x n e=0.2$ we used (Zakharov et al. 2007). At the end of the mission (30 September 2016, $r_{\mathrm{h}}=3.8 \mathrm{AU}$ ), the Rosetta Plasma Consortium experiments were able to measure the ion density 


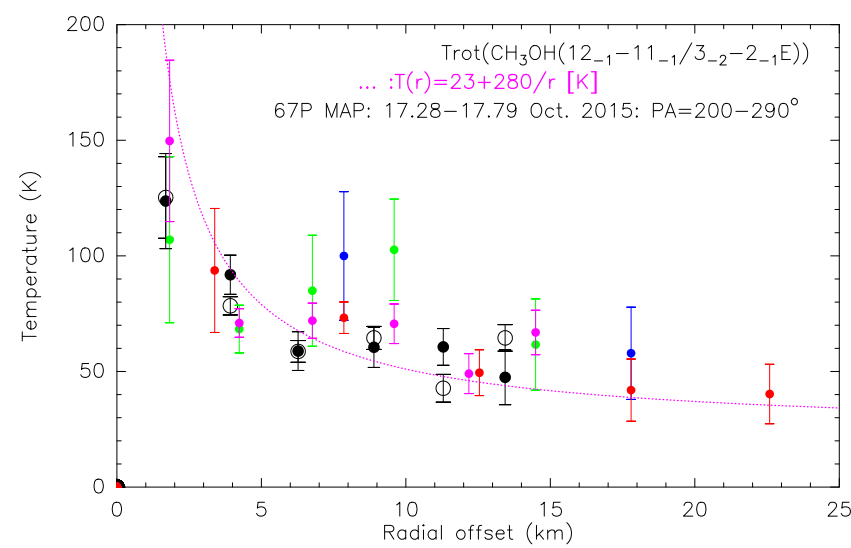

Fig. 6. Example of a radial profile of the rotational temperature $T_{\text {rot }}\left(\mathrm{CH}_{3} \mathrm{OH}\right)$ as a function of projected distance $\rho$ from the nucleus in the jet (see text). The empty circles correspond to the entire line profile, and the blue, green, black, red, and pink dots correspond to rotational temperatures measured in bins of Doppler velocity of $(-\mathrm{V} ;-0.6 \mathrm{~V})$, $(-0.6 V ;-0.2 V),(-0.2 V ;+0.2 V),(+0.2 V ;+0.6 V)$, and $(+0.6 V ;+V)$, respectively, where $V$ is either the average expansion velocity or half the FWHM of the line. Each velocity bin samples gas either closer to $\left(v_{i}<0\right)$ or farther away $\left(v_{i}>0\right)$ from the plane of the sky, i.e., at a cometocentric distance $r$ larger than the projected distance $\rho: r=\frac{\rho}{\cos (\psi)}$, where $\sin (\psi)=\frac{\langle v\rangle}{v_{\text {exp }}}$. These dots are plotted at the corresponding deprojected radii $r$ of $\pm 1.667 \rho, \pm 1.091 \rho$ or $\rho$.

between $20 \mathrm{~km}$ and the surface of the comet (Heritier et al. 2017). The density roughly follows $n=650 / r$ between $r=16$ and $5 \mathrm{~km}$ from the center of the comet. From our estimated water production rate $\left(1.2 \times 10^{24}\right.$ molec. $\mathrm{s}^{-1}$ in $\pi$ steradians $)$ and expansion velocity $\left(0.45 \mathrm{~km} \mathrm{~s}^{-1}\right)$ determined on 27 September 2016, our modeled (cold) electron density close to the nucleus Zakharov et al. (2007; where it is proportional to $1 / r$ ) yields $n_{e}(r) \approx 2400 / r$, using $x n e=0.2$. This is somewhat higher than the observed value, but low enough to affect the excitation of water in no great way; therefore it is compatible with observations. Unfortunately, there are few or no (in situ) RPC observation of ion or cold electron densities for higher activity in the regions we probe with MIRO observations.

\subsubsection{Temperature profile}

Corrections were made to convert the above retrieved temperatures into gas temperature radial profiles $T(r)$. The main-beam brightness temperature $T_{\mathrm{p}}\left(\mathrm{H}_{2}^{16} \mathrm{O}\right)$ corresponds to the RayleighJeans approximation of the blackbody radiation, which is slightly lower than the blackbody temperature. The correction required to obtain a physical temperature at the distance $r$, taking into account that part of the signal comes from the side lobes, is

$T\left(\mathrm{H}_{2}^{16} \mathrm{O}\right)(r) \approx T_{\mathrm{p}}\left(\mathrm{H}_{2}^{16} \mathrm{O}\right)(\rho)+10 \mathrm{~K}$.

We described the temperature profile $T(r)$ by the law $a+b / r$ that best fits the measurements obtained for $T_{\text {rot }}\left(\mathrm{CH}_{3} \mathrm{OH}\right)(r)$ and $T\left(\mathrm{H}_{2}^{16} \mathrm{O}\right)(r)$. Figures 6 and 7 show an example of radial profiles of $T_{\text {rot }}\left(\mathrm{CH}_{3} \mathrm{OH}\right), T_{\mathrm{p}}\left(\mathrm{H}_{2}^{16} \mathrm{O}\right)$, and $T(r)$. The values for $T(r)$ are provided in Table A.2. Maps of $T_{\mathrm{p}}\left(\mathrm{H}_{2}^{16} \mathrm{O}\right)$ (measured at the Doppler velocity $\sim+0.2-0.6 \mathrm{~km} \mathrm{~s}^{-1}$ ) are also shown in Fig. C.1.

In the higher density part of the coma we generally achieve a good fit of the data with the law $T(r)=a+b / r$, the decrease
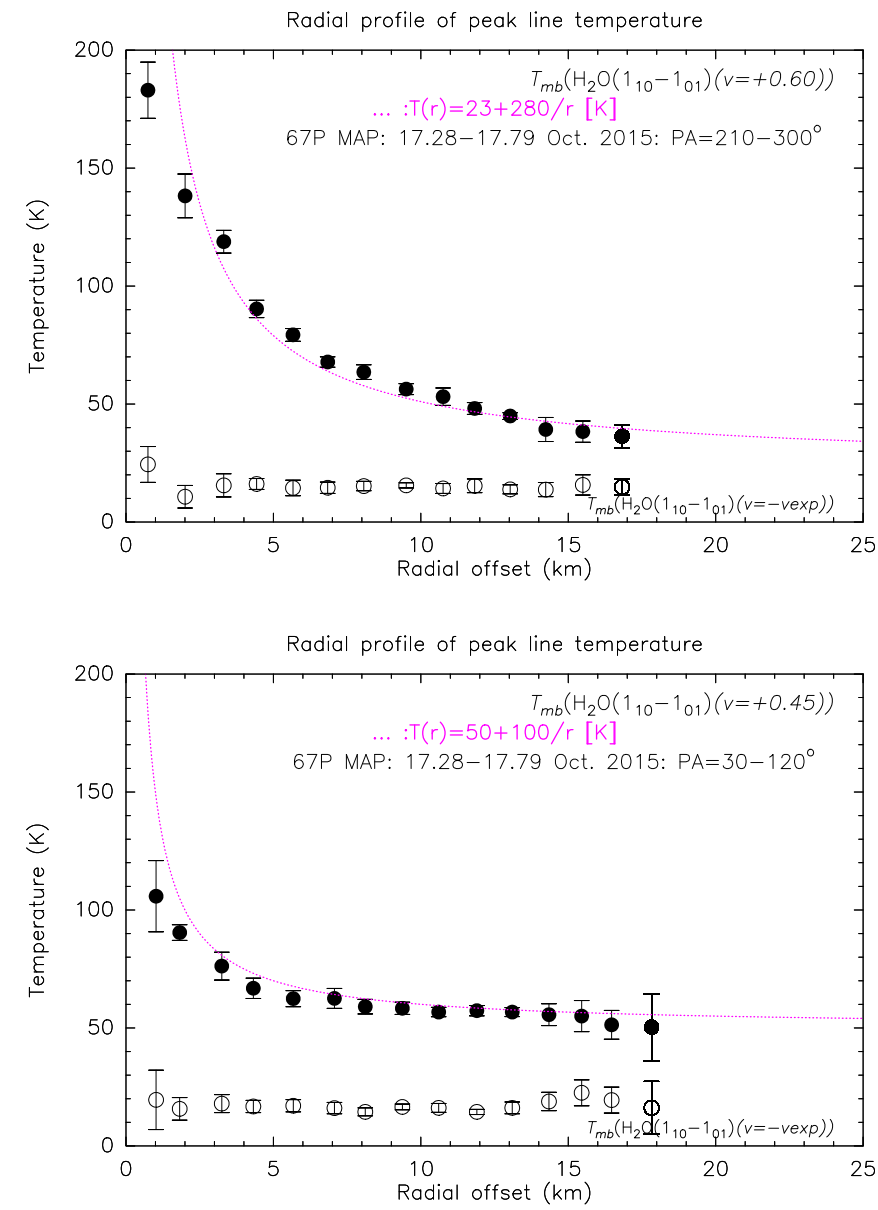

Fig. 7. Top panel: example of a radial profile of the peak intensity $T_{\mathrm{mb}}$ of the saturated $\mathrm{H}_{2}^{16} \mathrm{O}$ line at channel $v_{i} \sim+0.6 \mathrm{~km} \mathrm{~s}^{-1}$ as a function of projected distance $\rho$ from the nucleus in the jet (full circles). The empty circles correspond to the $T_{\mathrm{mb}}$ value for channels at $v_{i} \sim-v_{\mathrm{exp}}$, which saturate close to the gas temperature at the distance of the spacecraft. The dotted line shows the adopted gas temperature profile (see text). Bottom panel: same as above for the direction opposite to the jet direction (i.e., night side), using here channels at $+0.45 \mathrm{~km} \mathrm{~s}^{-1}$. Higher values a few kilometers from the nucleus can be interpreted as due to a lower efficiency of the cooling of the gas through adiabatic expansion.

as $1 / r$ being expected from adiabatic cooling. On the night side, $T\left(\mathrm{H}_{2}^{16} \mathrm{O}\right)(r)$ quickly stops to decrease with $1 / r$, likely because of inefficient adiabatic cooling (and possibly because of some warming by gas-decoupled hot electrons). We therefore obtain a warmer temperature profile for the low-density part of the coma, as is shown in several temperature maps of Appendix C, where the "warm" gas on the night side is more localized in the low-density anti-jet direction than in the anti-sunward direction.

To analyze nadir observations, we have limited constraints on the temperature profile along the line of sight. We assumed a $1 / r$ profile as above, constraining the $a$ and $b$ parameters from two measurements: (1) the temperature near the spacecraft $T(\Delta)$, which is derived from the $\mathrm{H}_{2}^{16} \mathrm{O}$ line profile at the velocity channel $v_{i}=-v_{\exp }$ (see above); for low activity cases, this value will be an upper limit to $T(\Delta)$; (2) the other constraint is from the maximum intensity at positive velocities, which is close to the background temperature $T_{\mathrm{bg}}$ of the nucleus and provides a constraint on the gas temperature near the surface. 


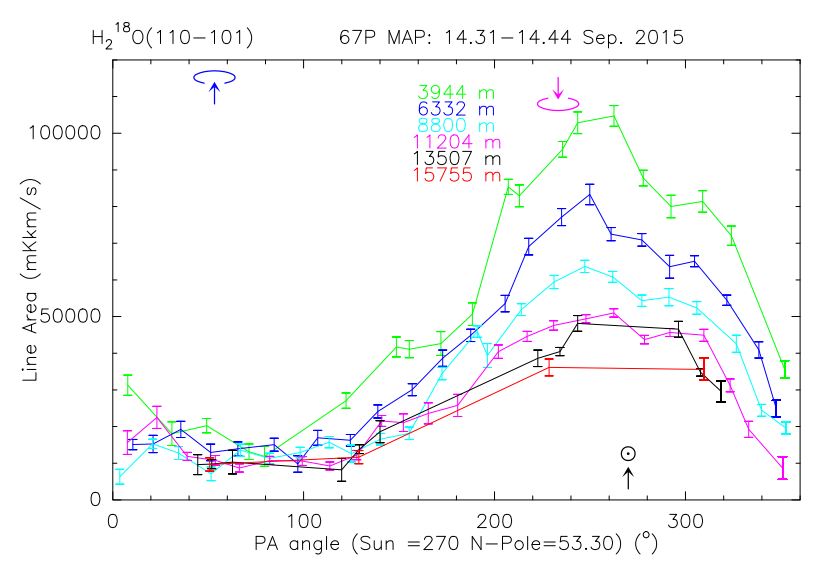

Fig. 8. Illustration of step 1 of the data analysis: azimuthal cut of the 14.31-14.44 September 2015 UT map of $\mathrm{H}_{2}^{18} \mathrm{O}$. The line-integrated intensity for lines averaged in bins of $15^{\circ}$ in azimuth (PA scale on the horizontal axis) and $2.5 \mathrm{~km}$ in projected radius is plotted for each PA and with a different color for each range of radial distance. The projected directions of the Sun, north pole (blue), and south pole (magenta) are indicated.

\section{Conversion of the 3D maps into a 2D outgassing pattern}

\subsection{Method}

The maps of the various molecular lines obtained with the MIRO spectrometer contain 3D information on the outgassing pattern: 2D spatial information in the plane of the sky (in $X$ and $Y$ directions defined by the Rosetta platform axis, with the $Z$-axis corresponding to the comet-Rosetta axis), plus Doppler velocity information. This velocity information can be converted into spatial information along the $Z$-axis because the Doppler velocity components of the line are the result of the projection on the line of sight of the velocity vector. We used spherical coordinates defined by the azimuthal angle PA (equal to $0^{\circ}$ along the vertical $+Y$-axis) and the colatitude angle $\phi$ (Table 2 and Fig. 4$)$. The various steps undertaken to derive information on the outgassing pattern and outgassing rate of each molecule are listed below.

1. Extract azimuthal cuts of the line intensity from the maps. We used steps of $2.5 \mathrm{~km} \times 15^{\circ}\left(20^{\circ}\right.$ for methanol or ammonia, depending on the $\mathrm{S} / \mathrm{N}$ ). An example is shown in Fig. 8.

2. Fit a Gaussian profile to the azimuthal variation of the line area $A(\mathrm{PA})$ for each radial distance to derive the projected main direction of the outgassing $\left(\mathrm{PA}_{\mathrm{jet}}\right)$, the projected angular width of the jet $\theta_{\text {proj }}$ from the FWHM of the Gaussian, the peak line area $A_{\mathrm{p}}$, and the background value $A_{0}$, with

$$
A(\mathrm{PA})=A_{0}+A_{\mathrm{p}} \times 2^{-\left(2^{\frac{\mathrm{PA}^{\mathrm{PPA}} J}{\mathrm{proj}}}\right)^{2}} .
$$

Then, the average values $\left\langle\mathrm{PA}_{\text {jet }}\right\rangle$ and $\left\langle\theta_{\text {proj }}\right\rangle$ over the different radial distances were computed.

3. Average all the on-nucleus (nadir) integrations for each line when available. We generally selected observations with a background continuum level higher than 130-160 K, depending on the average submillimeter continuum level around that date. When too few integrations on the nucleus were available during the mapping, we considered those obtained between two consecutive maps of the same day or just before or after. The line-integrated area measured in absorption was converted into an outgassing rate per solid angle $\left(q_{\text {nadir }}=q(\phi=0)\right)$.
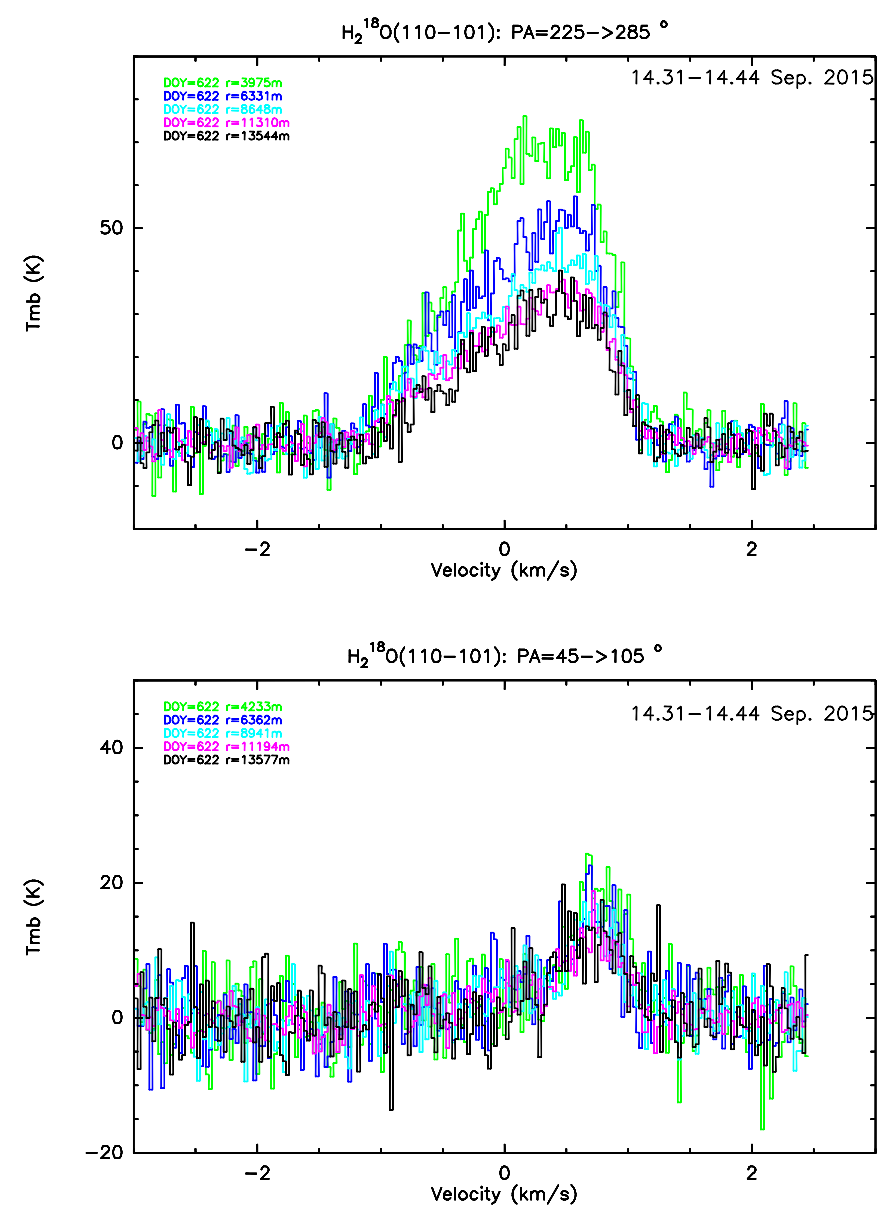

Fig. 9. Illustration of step 4 of data analysis. Top panel: jet. Extracted spectra of $\mathrm{H}_{2}^{18} \mathrm{O}$ from the 14.31-14.44 September 2015 UT map: averaged spectra in the $[225-285]^{\circ}$ range of PA and by step of $\approx 2.5 \mathrm{~km}$ in projected radius. The horizontal scale is Doppler velocity relative to the nucleus and the vertical scale line intensity in $T_{\mathrm{mb}}$ scale. Bottom panel: night side. Same as above, but for the $[45-105]^{\circ}$ range of PA.

4. Extract line profiles along the jet axis for a range of radii (step of $2.5 \mathrm{~km}, r>2.5 \mathrm{~km}$ ). We averaged spectra within $\mathrm{PA}=\mathrm{PA}_{\text {jet }} \pm 30^{\circ}$, and within $\mathrm{PA}=\left(\mathrm{PA}_{\text {jet }}+180^{\circ}\right) \pm 30^{\circ}$ (i.e., in the "night" side). This provided us with information on the distribution of the molecules within the jet as a function of the colatitude $\phi$. Examples are shown in Fig. 9.

5. In this step we performed what we call an "inversion" of the line profile to convert the intensity profile $T_{\mathrm{mb}}\left(v_{i}\right)$ into a production rate (per solid angle) profile $q(\phi)$. From simulated line profiles obtained using derived $v_{\exp }$ and $T(r)$ for both the jet and the night side (Sect. 3), the extracted line profiles were inverted into outgassing rate per solid angle $q(\phi)$ as a function of the colatitude angle $\phi$ in the plane defined by the $Z$-axis and jet main axis (with $0<\phi<180^{\circ}$ on the jet side and $180<\phi<360^{\circ}$ on the night side). For radial flow at a constant velocity $v_{\text {exp }}$, each velocity channel $v_{i}$ of the line approximately corresponds to gas that expands in the direction $\phi_{i}$ so that $v_{i}=v_{\exp } \cos \left(\phi_{i}\right)$. Simulations show that as a result of thermal broadening, the contribution for each projected velocity spreads over 0.37 to $0.43 \mathrm{~km} \mathrm{~s}^{-1}$ (FWHM), which is taken into account for the inversion. Angular production rates $q_{i}(\phi)$ derived from nearby channels are thus correlated. First-order non-linearity due to opacity is also taken into account. The estimated noise on each line channel 

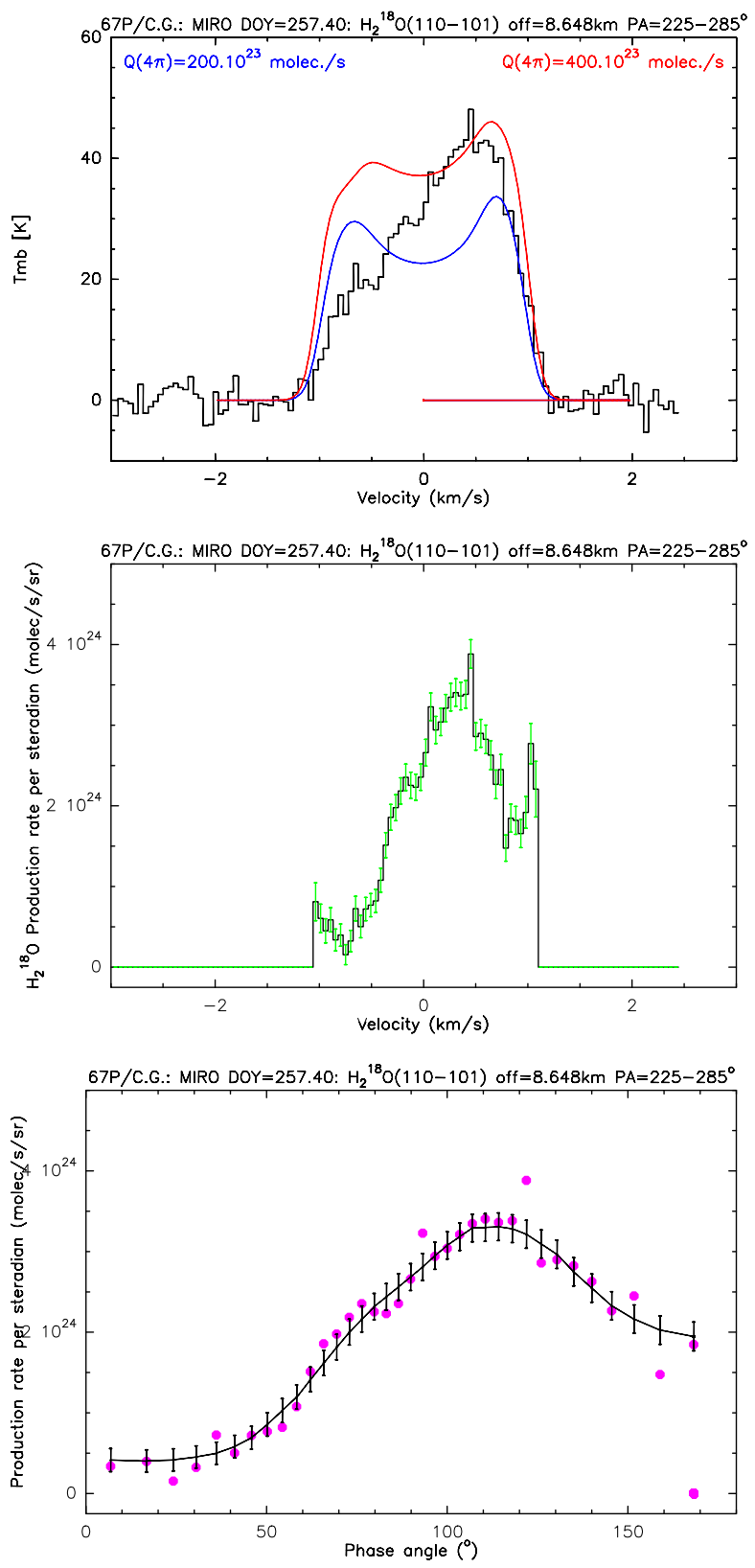

Fig. 10. Illustration of step 5 of data analysis for the jet. Top panel: extracted spectra of $\mathrm{H}_{2}^{18} \mathrm{O}$ from the 14.31-14.44 September 2015 UT map in the [225-285] $]^{\circ}$ range of PA and [7.5-10] km range of the projected radius (mean radius of $8.648 \mathrm{~km}$, middle spectrum from Fig. 9) Isotropic models with $Q_{\mathrm{H}_{2}^{18} \mathrm{O}}=2 \times 10^{25}$ molec. $\mathrm{s}^{-1}$ (blue) and $Q_{\mathrm{H}_{2}^{18} \mathrm{O}}=$ $4 \times 10^{25}$ molec. $\mathrm{s}^{-1}(\mathrm{red})$ are superimposed for channel-by-channel interpolation and determination of $q_{\mathrm{H}_{2}^{18} \mathrm{O}}$ for each channel. Central panel: line intensity in each channel or velocity bin converted into production rate per solid angle (vertical scale). There is a sharp cutoff for absolute velocities that are higher than the expansion velocity, for which the signal is close to 0, as expected. Bottom panel: horizontal scale in Doppler velocity converted into colatitude $\phi$ angle $\left(v_{i}=v_{\exp } \cos \left(\phi_{i}\right)\right)$ assuming a constant expansion velocity ( $v_{\text {exp }}=0.82 \mathrm{~km} \mathrm{~s}^{-1}$ here). Pink dots show values that were retrieved from individual channels, and connected points represent a smoothed profile that takes thermal broadening into account (see text).

is used to estimate the uncertainty on the derived values. Figures 10-12 show the step-by-step process on one example of spectrum.
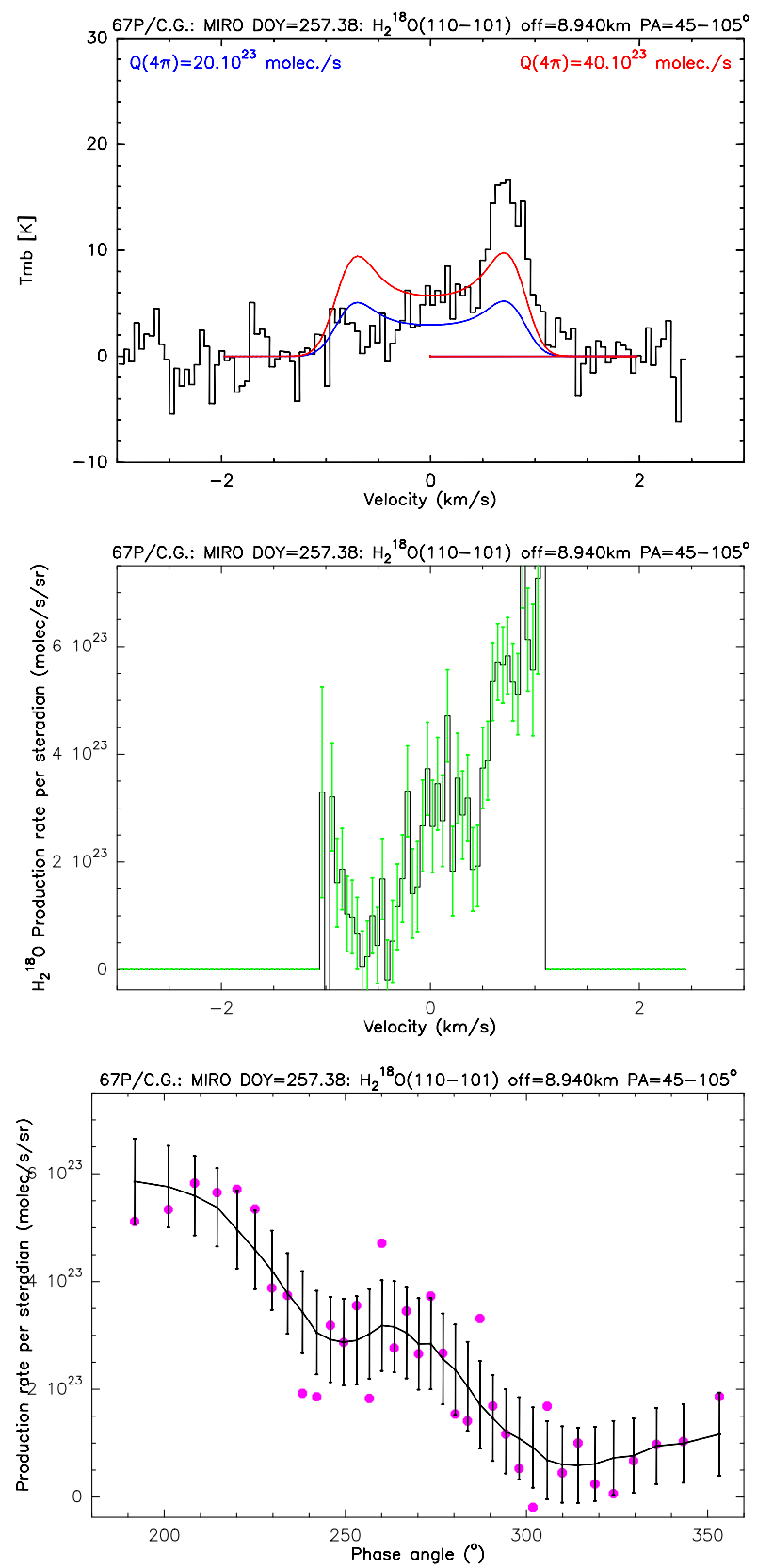

Fig. 11. Same as Fig. 10 for the night side. Extracted spectra of $\mathrm{H}_{2}^{18} \mathrm{O}$ from the 14.31-14.44 September 2015 UT map, in the [45$105]^{\circ}$ range of PA and [7.5-10] $\mathrm{km}$ range of projected radius (mean radius of $8.941 \mathrm{~km}$, middle spectrum from Fig. 9). Top panel: isotropic models correspond to $Q_{\mathrm{H}_{2}^{18} \mathrm{O}}=2 \times 10^{24}$ molec. $\mathrm{s}^{-1}$ (blue) and $Q_{\mathrm{H}_{2}^{18} \mathrm{O}}=$ $4 \times 10^{24}$ molec. $\mathrm{s}^{-1}$ (red).

6. For each range of radial distances, a Gaussian shape was fit to the $q(\phi)$ profiles to derive the following four parameters: $q_{\mathrm{I}, \phi}$ (background level of the Gaussian, corresponding to the isotropic component of the outgassing), $q_{\mathrm{p}}$ (peak value of $q(\phi)), \phi_{J}$ (direction of the maximum), and $\theta_{\phi}$ (the FWHM). An example of the results is shown in Fig. 13. Because the signal was generally averaged over $60^{\circ}$ in azimuth, the true peak outgassing rate needed to be corrected: $q_{J, \phi}=q_{\mathrm{p}} / 0.93$ for a Gaussian distribution with $F W H M \approx 105^{\circ}$ (the exact correction was computed according to the actual $\left.\theta_{\text {proj }}\right)$. Then, a weighted (according to error bars) average was computed over the various radii. In the absence of distributed sources 


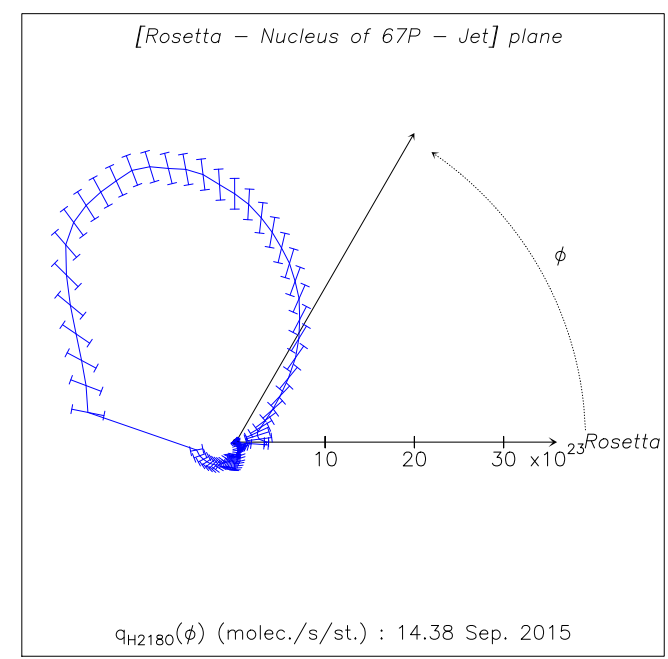

Fig. 12. Illustration of step 5 of data analysis. Polar plot representation of the derived angular production rate $q(\phi)$ for the $7.5-10 \mathrm{~km}$ projected radius range. The maximum of production $\left(q=3.5 \times 10^{24}\right.$ molec. $\mathrm{s}^{-1} \mathrm{sr}^{-1}$ ) is towards a colatitude angle of $\approx 110^{\circ}$, slightly away from the direction of Rosetta.

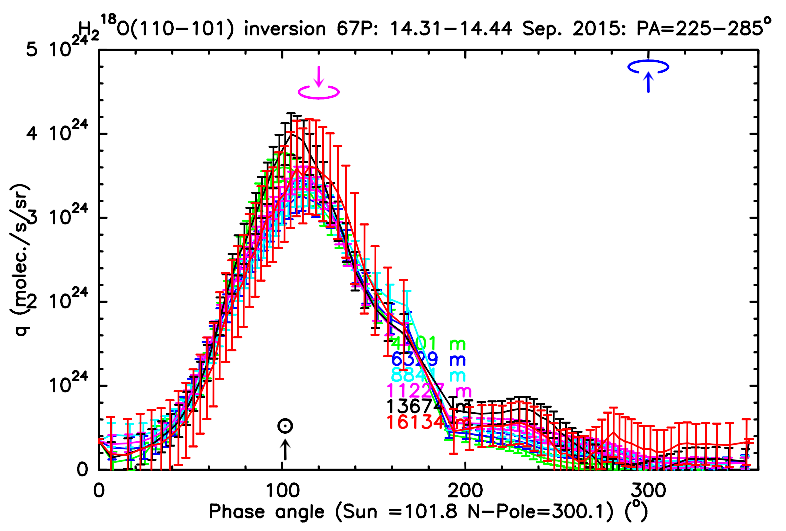

Fig. 13. Step 6: combining results of step 5 for each range of projected radius. Derived angular production rate $q(\phi)$ for the $\mathrm{H}_{2}^{18} \mathrm{O}$ map obtained on 14.31-14.44 September 2015.

of gases or significant time variability, the four parameters, $q_{\mathrm{J}, \phi}, q_{\mathrm{I}, \phi}, \phi_{\mathrm{J}}$ and $\theta_{\phi}$ should not depend on the radii.

7. We can now use $\left\langle\phi_{\mathrm{J}}\right\rangle$ to correct the effect of projecting a $2 \mathrm{D}$ Gaussian on the plane of the sky. From the derived Gaussianlike profile $A(\mathrm{PA}$; Eq. (6)), and the angle with the line of sight $\left\langle\phi_{\mathrm{J}}\right\rangle$, we determined the real width of the Gaussian $\theta_{\mathrm{az}}$ and the effective contributions $A_{\mathrm{J}}$ and $A_{\mathrm{I}}\left(A_{\mathrm{J}}+A_{\mathrm{I}}=A_{\mathrm{p}}+A_{0}\right)$ of the jet and isotropic components to the signal. We used a code that computes the projection of a 2D Gaussian and provides the correction factor $\epsilon$ needed to compute $A_{\mathrm{J}}=\frac{A_{\mathrm{p}}}{1-\epsilon}$.

8. The solid angle $\Omega_{\text {jet }}$ of the jet is then computed using its average $F W H M: \theta_{J}=\sqrt{\theta_{\phi} \times \theta_{\mathrm{az}}}$. We found values of $\Omega_{\text {jet }}$ ranging from 0.5 to $1.5 \pi$. Uncertainties on the determined $\theta$ angles were propagated to $\Omega_{\text {jet }}$ and to the total production rate in the jet $Q_{\mathrm{J}}$.

9. We used our 1D radiative transfer code to simulate this jet (Sect. 3.2) taking into account the orientation $\left\langle\phi_{\mathrm{J}}\right\rangle$ of the jet with respect to the line of sight. This allowed us first to check that the mean Doppler shift of the line was well reproduced (especially for weak lines for which the determination of $\left\langle\phi_{\mathrm{J}}\right\rangle$ was not possible, such as the $\mathrm{H}_{2}^{17} \mathrm{O}$ line). This simulation was used to convert $A_{\mathrm{J}}$ into outgassing rate $q_{J, a z} . \Omega$ was chosen as close as possible to the measured $\Omega_{\text {jet }}$ to avoid misestimating $q_{\mathrm{J}, \mathrm{az}}$. The comparison with $q_{\mathrm{J}, \phi}$ generally confirms this. The isotropic component $q_{\mathrm{I}, \mathrm{az}}$ was also derived from $A_{\mathrm{I}}$. The assumption that the total outgassing is the sum of two components (jet + isotropic) holds as long as opacity effects are not too high to depart from the linear domain. In most cases, however, the previous correction step shows that $A_{\mathrm{I}}$ is generally much closer to 0 than to $A_{0}$, hence that most of the outgassing is contained in a Gaussian-like jet. In the case of strong opacity effects (e.g., $\mathrm{H}_{2}^{16} \mathrm{O}$ on 29 July 2014), we simply considered the "isotropic" component only outside the jet (in $4 \pi-\Omega_{\text {jet }}$ steradians instead of $4 \pi$ ) and used the whole line intensity inside the jet to compute the production rate inside the jet to avoid non-linearity problems. We then averaged over the various radii to obtain $\left\langle q_{\mathrm{I}, \mathrm{az}}\right\rangle$ and $\left\langle q_{\mathrm{J}, \mathrm{az}}\right\rangle$.

10. The last step is to consider the two perpendicular components (az and $\phi$ ) to derive the average global values: the peak outgassing rate per solid angle in the jet,

$q_{\mathrm{J}}=\left(\left\langle q_{\mathrm{J}, \mathrm{az}}\right\rangle+\left\langle q_{\mathrm{J}, \phi}\right\rangle\right) / 2$,

the isotropic component,

$q_{\mathrm{I}}=\left(\left\langle q_{\mathrm{I}, \mathrm{az}}\right\rangle+\left\langle q_{\mathrm{I}, \phi}\right\rangle\right) / 2$,

and the corresponding total outgassing rate,

$Q=Q_{\mathrm{I}}+Q_{\mathrm{J}}=4 \pi q_{\mathrm{I}}+\Omega_{\mathrm{jet}} q_{\mathrm{J}}$

The process described above can be applied as long as the S/N allows for $\mathrm{H}_{2}^{18} \mathrm{O}, \mathrm{CH}_{3} \mathrm{OH}$, and $\mathrm{CO}$, but the line inversion is difficult for $\mathrm{NH}_{3}$ and $\mathrm{H}_{2}^{17} \mathrm{O}$ because of their hyperfine structure (Table 3, see examples of spectra in Fig. E.1). Details on the inversion of the $\mathrm{NH}_{3}$ line are given in Sect.4.2.

Values of the total production rates $Q$, production rates within the Gaussian jet $Q_{\mathrm{J}}$ and in the isotropic component $Q_{\mathrm{I}}$ are provided in Table A.3. The formal uncertainties are $1 \sigma$ : they are based on the weighted average of measurements of $q$ at different nucleus distances, and the (quadratic) sum of the uncertainties on each component (isotropic and jet production rate) and on the jet solid angle. The uncertainty on $Q$ might appear larger than $Q$ itself in some case although the line is clearly detected in the jet part. The decomposition of signal into two contributions from a jet and isotropic outgassing might bring a large uncertainty as a result of the undetected isotropic contribution in such cases. For detections with a low $\mathrm{S} / \mathrm{N}$, considering only the jet contribution and its uncertainty can yield a more realistic estimate of the total outgassing rate and its uncertainty.

We provide in Table A.4 the derived production rates in the jet $Q_{\text {jet only }}$ that we obtained when we did not consider any isotropic counterpart. They were obtained by fitting the line intensities along the main jet direction (extractions made in step 4), using the radiative transfer simulations that only consider a jet contribution (Sect. 3.2). Values of $Q_{\text {jet only }}$ have smaller uncertainties than $Q$ values, and their relative uncertainties are more representative of the quality of the line detections. They were used to compute relative molecular abundances in the jet (Sect. 10).

Line inversion was possible for $\mathrm{NH}_{3}$ when the line was strong enough (Sect. 4.2). When line inversion could not be performed, so that $\left\langle\phi_{\mathrm{J}}\right\rangle$ values could not be derived (Step 6), we assumed the 
Table 3. $F_{1}$ Hyperfine components of the $\mathrm{H}_{2}^{17} \mathrm{O}$ and $\mathrm{NH}_{3}$ lines observed by MIRO.

\begin{tabular}{lcclcr}
\hline \hline & $F_{\mathrm{u}}$ & $F_{1}$ & $\begin{array}{l}\text { Frequency } \\
(\mathrm{MHz})\end{array}$ & $I$ & $\begin{array}{r}\Delta V \\
\mathrm{~km} \mathrm{~s}^{-1}\end{array}$ \\
\hline $\mathrm{H}_{2}^{17} \mathrm{O} 1_{10}-1_{01}$ & $3 / 2$ & $3 / 2$ & 552018.458 & 0.0666 & +1.437 \\
& $7 / 2$ & $7 / 2$ & 552020.137 & 0.2857 & +0.525 \\
& $3 / 2$ & $5 / 2$ & 552021.168 & 0.1555 & +0.035 \\
& $5 / 2$ & $3 / 2$ & 552021.443 & 0.1555 & -0.185 \\
& $7 / 2$ & $5 / 2$ & 552021.916 & 0.1587 & -0.441 \\
& $5 / 2$ & $7 / 2$ & 552022.381 & 0.1588 & -1.021 \\
& $5 / 2$ & $5 / 2$ & 552024.159 & 0.0190 & -1.660 \\
$\mathrm{NH}_{3}(1-0)$ & 1 & 1 & 572497.130 & 0.3333 & +0.650 \\
& 2 & 1 & 572498.371 & 0.5555 & 0.000 \\
& 0 & 1 & 572500.191 & 0.1111 & -0.953 \\
\hline
\end{tabular}

Notes. $I$ is the relative intensity of the hyperfine component. $\Delta V$ is the velocity offset from the line centroid. $\mathrm{H}_{2}^{17} \mathrm{O}$ data from Puzzarini et al. (2009) and Puzzarini (priv. comm.). $\mathrm{NH}_{3}$ data from Cazzoli et al. (2009).

value of $\left\langle\phi_{\mathrm{J}}\right\rangle$ determined for $\mathrm{H}_{2}^{18} \mathrm{O}$ or $\mathrm{CH}_{3} \mathrm{OH}\left(3_{-2}-2_{-1} E\right)$. For data with low $\mathrm{S} / \mathrm{N}$, we observed that considering the isotropic component often added a significant uncertainty on the total outgassing rate because of its wide $(4 \pi)$ extent. This often resulted in an error bar for the retrieved total production rate $Q$ that was larger than the value itself. On the other hand, the uncertainties on $Q$ are conservative because the determinations of $Q_{\mathrm{I}}$ and $Q_{\mathrm{J}}$ are not independent.

\subsection{Deconvolution of the ammonia line profile}

The ammonia line consists of three hyperfine components, with statistical weights of $1 / 3,5 / 9$, and $1 / 9$, which partly overlap (Table 3). In order to retrieve the jet profile along the $\phi$ angle, the line profile needs to be deconvolved to infer the Doppler profile of $\mathrm{NH}_{3}$. This was possible for data acquired from June to October 2015, when the ammonia line was strong enough. We adopted a simplified approach because the $F(0-1)$ component at $-0.953 \mathrm{~km} \mathrm{~s}^{-1}$ and the $F(1-1)$ component at $+0.650 \mathrm{~km} \mathrm{~s}^{-1}$ partly do not overlap the main $F(2-1)$ hyperfine component. In the simple case of an optically thin line and a Doppler line width smaller than $1.6 \mathrm{~km} \mathrm{~s}^{-1}$, the blueshifted (not overlapping) part of $F(0-1)$ component and the redshifted part of the $F(1-1)$ component can be used to retrieve the Doppler profile of each single component, as illustrated in the upper plot of Fig. 14. In practice, we retrieved the profile of the strongest (most opaque) $F(2-1)$ hyperfine component by subtracting the estimated overlapping parts of the weaker components. For broad lines, we used an iterative process to correctly retrieve the contribution of each component to the full line, resulting in a retrieved $F(2-1)$ line profile with a noise increased by a factor $1.3-4.3$ depending on the channels. To cope with opacity effects, which only affect the main component, we introduced correcting factors in the iterative process. Figure 14 illustrates the deconvolution for optically thin and optically thick synthetic spectra. The major caveat of the method is that a dip appears in the central part of the line for optically thick cases. However, the line width and position are correctly retrieved and the line area is preserved, therefore we expect that the retrieved outgassing profile is not significantly affected.
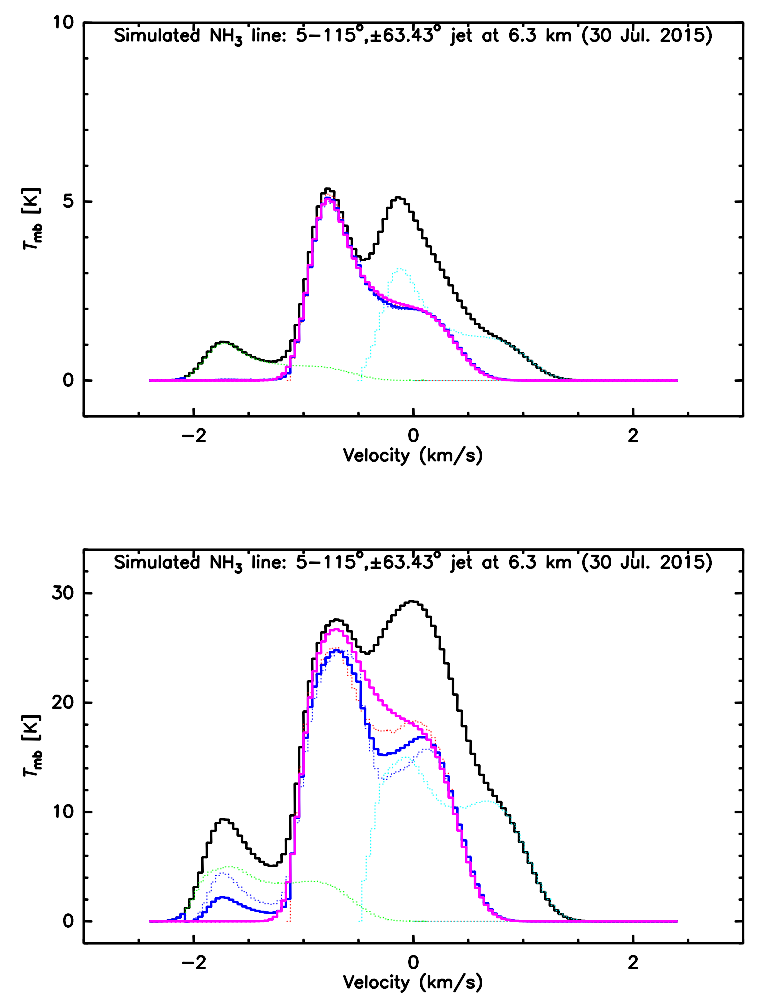

67P/C.G.: $\mathrm{NH}_{3}(1-0)$ at $572 \mathrm{GHz}: 30.31-30.65$ July 2015

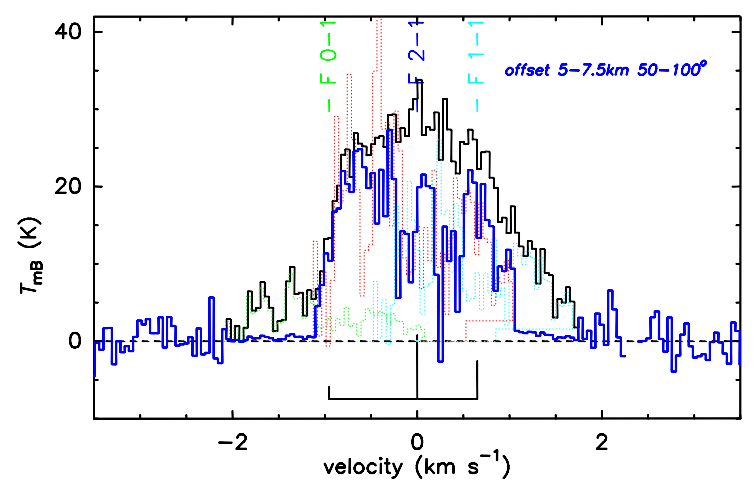

Fig. 14. Top panel: simulated optically thin $\left(Q_{\mathrm{NH}_{3}}=\right.$ $2.25 \times 10^{24}$ molec. $\mathrm{s}^{-1}$ ) $\mathrm{NH}_{3}$ line (in black); the Doppler profile of the $F(2-1)$ component is shown in pink. The dotted lines show the estimated $F(0-1)$ (green), $F(1-1)$ (light blue), and $F(2-1)$ (red) profiles by the deconvolution routine. The adopted retrieved profile for the $F(2-1)$ component alone from the deconvolution process is shown in bold dark blue. This is the average of the subtraction result of the estimated $F(0-1)$ and $F(1-1)$ profile from the full line and estimated $F(2-1)$ (red) profile. Middle panel: same as above, but for $Q_{\mathrm{NH}_{3}}=22.5 \times 10^{24}$ molec. $\mathrm{s}^{-1}$ with strong optical effects, which results in slight differences between the retrieved bold dark blue profile and the simulated pink profile. This corresponds to the highest opacity case encountered for this line. Bottom panel: $\mathrm{NH}_{3}$ line observed on 30.31-30.65 July 2015 (black), and the deconvolved Doppler profiles of the hyperfine components (same color coding as above plots). The position and relative intensities of the three hyperfine components are indicated below the line, and their identification is indicated above.

The results of this analysis are presented in Table A.3, which provides the retrieved jet characteristics and production rates for each map and molecule. In addition, the column densities measured along the jet axis are provided in Appendix D for each day. 

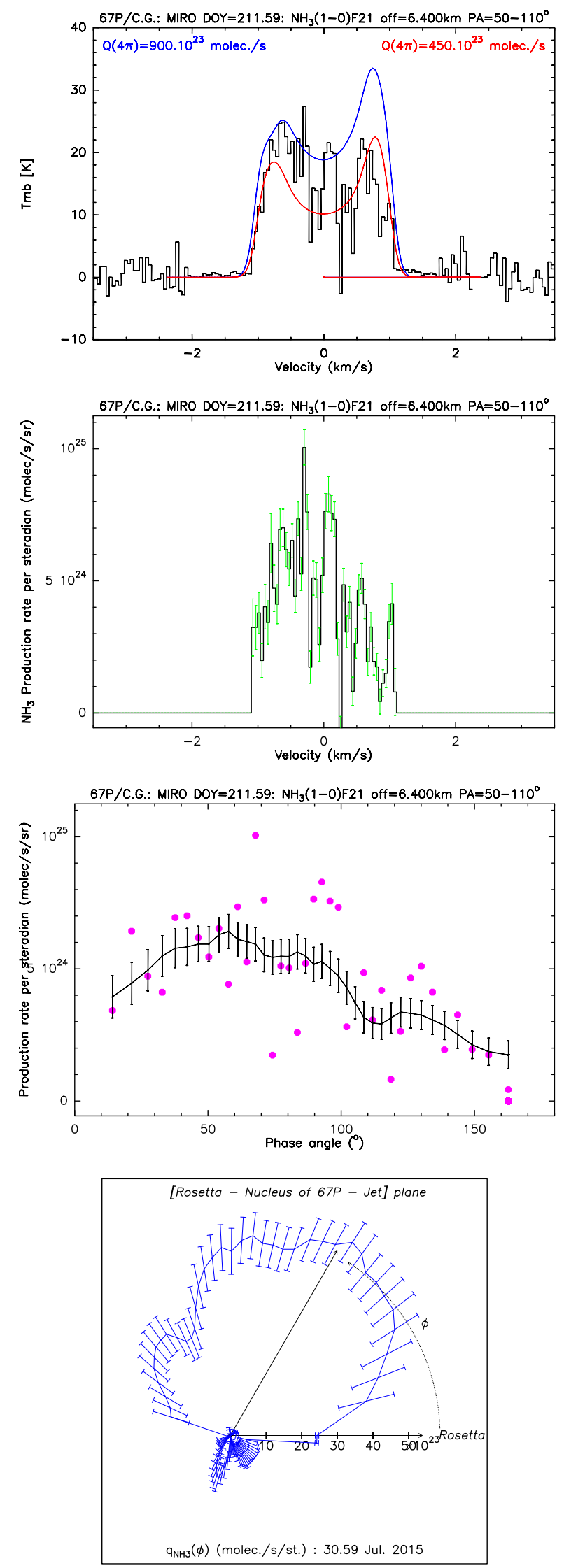

Fig. 15. Illustration of step 5 of the data analysis for a deconvolved $\mathrm{NH}_{3}$ line profile. See the caption of Fig. 10 for the three upper plots, and the caption of Fig. 12 for the bottom plot.
The column densities are more directly comparable to measurements obtained by other remote instruments such as VIRTIS and Alice.

\section{Uncertainties and biases on the retrieved outgassing rates}

\subsection{Smearing of the maps through long integrations}

Maps were acquired in typically 3-4 h (Table A.1), during which the nucleus rotated by $90^{\circ}-120^{\circ}$. This affects our determination of the gas jet properties. If it rotated by this amount, the maps and line profiles do not reflect the effective angular extent of the jet. However, in practice, our analysis is not significantly affected by this effect: (i) the actual time spent to scan the jet itself is shorter than for the total map; (ii) if the jet is rotating in the plane of the sky, it should appear broader when we scan in the direction of motion and narrower in the opposite direction: this should average out or show some systematic effects; (iii) for the jet transverse profile width deduced from line profile, we used a subsample of integrations that were acquired over an even shorter timescale; (iv) as shown in Table A.3, when several consecutive maps of $\mathrm{H}_{2}^{18} \mathrm{O}$ were reduced separately, the motion of the jet between two maps is generally small (less than $20^{\circ}$ ), which means that the smearing is not significant. Finally, we find that the outgassing pattern is not instantaneously connected to the surface topography of the nucleus, but rather to the illumination, and it depends more on the Sun and pole directions, which do not change much during the mapping.

\subsection{Effect of the uncertainty on the expansion velocity}

The terminal expansion velocity $v_{\text {exp }}$ is readily determined from the absorption peak in lines against the nucleus: this provides us with the value on the line of sight. For this measurement, the uncertainty is generally below $0.01 \mathrm{~km} \mathrm{~s}^{-1}$ and has negligible effect on final results. There is evidence that the expansion velocity varies both radially $\left(v_{\exp }(r)\right)$ and laterally, however. For the radial variation, we could have used a two-parameter law (e.g. as in Biver et al. 2011), but this would only be necessary for the $\mathrm{H}_{2}^{16} \mathrm{O}$ optically thick line for the contribution at lower velocity close to the nucleus, which is not used to determine the water production. Nevertheless, close to perihelion, the "inversion" of the line profiles does require lower velocities close to the nucleus. In this case, we used different fixed values of $v_{\exp }(\rho)$ for projected distances below 5-10 km (e.g. $0.82 \mathrm{~km} \mathrm{~s}^{-1}$ below $8 \mathrm{~km}$, then 0.85 and $0.87 \mathrm{~km} \mathrm{~s}^{-1}$ beyond $10 \mathrm{~km}$ to invert the line profiles obtained on 14 September 2015). For the lateral variation of $v_{\text {exp }}$, we also clearly see that the expansion velocity is higher in the jet closer to subsolar point than elsewhere. For instance, on 14-26 September 2015, nadir pointing at phase angles (line-ofsight-jet angles) of $103^{\circ}\left(117^{\circ}\right), 90^{\circ}\left(110^{\circ}\right)$, and $54^{\circ}\left(50^{\circ}\right)$ yield values of $0.82,0.84$, and $0.90 \mathrm{~km} \mathrm{~s}^{-1}$ for $v_{\exp }$ on the line of sight. This means that the expansion velocity close to the axis of the jet is higher than is often measured on the line of sight (away from the jet). We tried to constrain $v_{\text {exp }}$ from the line profile itself while inverting the line. The derived value (Table A.2, Col. 3) is likely lower than the true value in the center of the jet, but more representative of the average velocity $v_{\exp }$ needed for a correct line profile inversion. Nevertheless, we estimated the effect of a $10 \%$ error on $v_{\text {exp }}$ : an underestimation by $10 \%$ results in an overestimation of the jet width $\theta_{\phi}$, and in an underestimation of the 
peak outgassing rates per solid angle $q_{\mathrm{J}, \phi}$ and $q_{\mathrm{J}, \mathrm{az}}$. In the end, the retrieved total production in the jet would be underestimated by $6 \%$. Because it is unlikely that we underestimated $v_{\exp }$ by more than $10 \%$, the underestimation of $Q$ is lower than $\sim 6 \%$.

\subsection{Effect of the uncertainty on the gas temperature}

We used a temperature law consistent with the derived methanol rotational temperatures and the peak temperatures of the saturated $\mathrm{H}_{2}^{16} \mathrm{O}$ line. However, the agreement is not always perfect, possibly because their rotational temperatures differ (e.g. due to collisional excitation effects). The $\mathrm{CH}_{3} \mathrm{OH}$ rotational temperature tends to show deviations from the assumed $1 / r$ profile, with slightly higher values locally in the $r=7-10 \mathrm{~km}$ range, for example, as shown in Fig. 6. Therefore, we explored the effect of a $20 \%$ increase in temperature along the jet. The test for the data of 11.6 November 2015 shows that the effect is negligible on the derived water production rate $(-1.5 \%)$ and small for methanol when the three lines $(+13 \%)$ are considered. For ammonia, increasing the gas temperature by $20 \%$ increases the production rate by $+19 \%$. Another test, using data of 8.7 September 2015, where we instead used temperatures higher by $\sim 8 \%$, did not change any of the final production rates by more than $2 \%$. CO is still less affected. We tested the effect for the observation on 30 August (nadir data) - 1 September 2015 (map) on retrieving the CO production rate of a temperature profile that decreases from the surface temperature to a terminal value between 10 and $200 \mathrm{~K}$ (extreme cases, $43 \mathrm{~K}$ being the adopted value from Table A.2). For the mapping data, the adopted temperature profile yields the lowest $\mathrm{CO}$ production rate, but a factor two in the terminal temperature will not increase the $\mathrm{CO}$ production by more than $10 \%$. Only the extreme case of a temperature profile would yield a 20-30\% increase in $\mathrm{CO}$ production. As a conclusion, the derived water and $\mathrm{CO}$ production rates do not depend much on the gas temperature, while those of methanol and ammonia could be slightly underestimated if we underestimate the gas temperature. For methanol, the observed behavior is strongly dependent on the lower energy level $\mathrm{CH}_{3} \mathrm{OH}\left(3_{-2}-2_{-1} E\right)$ line (Table 1$)$, which is much better detected than the two other transitions and has a greater weight in the computed average production rate. On average, $T+20 \%$ therefore implies same $Q_{\mathrm{H}_{2} \mathrm{O}}$ and $Q_{\mathrm{CO}}, Q_{\mathrm{CH}_{3} \mathrm{OH}}+10 \%$ and $Q_{\mathrm{NH}_{3}}+20 \%$.

\subsection{Opacity effects}

To invert the line profiles, opacity is taken into account to first order assuming that the line intensity is proportional to $Q^{1+x}$. The coefficient $x$ was determined using the radiative transfer model by computing the line area for two different production rates $Q$ that encompass the observed value.

We had to account for high opacity effects when we analyzed the $\mathrm{H}_{2}^{16} \mathrm{O}$ line (when $\mathrm{H}_{2}^{18} \mathrm{O}$ is too faint), or for the $\mathrm{H}_{2}^{18} \mathrm{O}$ line itself, or even sometimes around perihelion for the $\mathrm{NH}_{3}^{2}$ line. During the line inversion process, we did not consider the channels where optical thickness is so strong that our simplified model fails to reproduce their intensity. This particularly concerned channels at negative velocities, that is, sampling the gas along the line of sight near the spacecraft. For high optical depths $(\tau>1.0)$, the intensity starts to depend much more on the local temperature $T(r, \theta)$ and velocity gradient than on the column density, and a full 2D accurate modeling of $T(r, \theta), v_{\exp }(r, \theta)$ and $n(r, \theta)$ would be necessary. We note that by averaging the molecular lines in time and spatially, local variations in line opacities that are due to fine, denser structures in the jet, for instance, were possibly erased.

$\mathrm{H}_{2}^{16} \mathrm{O}, \mathrm{H}_{2}^{18} \mathrm{O}$, and $\mathrm{H}_{2}^{17} \mathrm{O}$ have a very different optical thickness. To study the $\mathrm{H}_{2} \mathrm{O}$ outgassing properties, we mostly used the $\mathrm{H}_{2}^{18} \mathrm{O}$ line, which is most of the time detected and not saturated $\left({ }^{16} \mathrm{O} /{ }^{18} \mathrm{O} \sim 500\right)$. Because of its complex hyperfine structure (Table 3), the $\mathrm{H}_{2}^{17} \mathrm{O}$ line cannot be easily used to invert the line profile. On the other hand, this line is on average ten times less optically thick for an ${ }^{18} \mathrm{O} /{ }^{17} \mathrm{O}$ isotopic ratio of 5.4. As we discussed above, the outgassing rates of $\mathrm{H}_{2}^{17} \mathrm{O}$ were retrieved using information on the transverse geometry of the outgassing obtained from either $\mathrm{H}_{2}^{18} \mathrm{O}$ or other molecules. The constancy of the derived $Q\left(\mathrm{H}_{2}^{18} \mathrm{O}\right) / Q\left(\mathrm{H}_{2}^{17} \mathrm{O}\right)$ ratio (Fig. 19, Sect. 9) indicates that opacity effects were well estimated for $\mathrm{H}_{2}^{18} \mathrm{O}$.

\section{5. ${ }^{16} \mathrm{O} /{ }^{18} \mathrm{O}$ isotopic ratio and water ortho-to-para ratio}

For more than $95 \%$ of the maps, we used the observation of the ortho line $J_{\mathrm{Ka}, \mathrm{Kc}}=1_{10}-1_{01}$ of $\mathrm{H}_{2}^{18} \mathrm{O}$ to retrieve the total outgassing rate of water. We assumed an $\mathrm{H}_{2}^{16} \mathrm{O} / \mathrm{H}_{2}^{18} \mathrm{O}$ ratio of 500 . This isotopic ratio is close to the Earth value (498.7), and is compatible with values observed in other comets (Biver et al. 2007, 2016; Bockelée-Morvan et al. 2012, 2015b). The Rosetta Orbiter Spectrometer for Ion and Neutral Analysis (ROSINA) mass spectrometer instrument and the cometary secondary ion mass analyser (COSIMA) with its time of flight mass spectrometer (TOF-SIMS) on board Rosetta have measured the ${ }^{16} \mathrm{O} /{ }^{18} \mathrm{O}$ in different materials: $555 \pm 62$ in $\mathrm{H}_{2} \mathrm{O}$ with ROSINA doublefocusing magnetic mass spectrometer (DFMS) in September 2014 (Altwegg et al. 2015), $445 \pm 35$ with ROSINA DFMS in $\mathrm{H}_{2} \mathrm{O}$ and $\mathrm{OH}$ by Schroeder et al. (2019) and $500 \pm 30$ in dust grains by Paquette et al. (2018). No observation suggests that this ratio departs by significantly more than $11 \%$ from the telluric value, which means that in the worst case, we might underestimate or overestimate the water outgassing rate by $11 \%$ for this reason.

We also assumed an ortho-to-para ratio (OPR) of 3. The OPR can be derived from infrared $\mathrm{H}_{2} \mathrm{O}$ spectra where several ro-vibrational lines of ortho and para species are observed simultaneously. Values measured in cometary $\mathrm{H}_{2}^{16} \mathrm{O}$ range from $\sim 2.5$ to 3.0 (Bockelée-Morvan et al. 2004; Mumma \& Charnley 2011). The OPR of $\mathrm{H}_{2}^{18} \mathrm{O}$, which has not been yet measured, is presumably similar to that of $\mathrm{H}_{2}^{16} \mathrm{O}$. If the OPR is lower, for example, 2.5 instead of 3 , then we underestimate the total water production rate by a factor $(3.5 / 2.5) /(4 / 3)=1.05$, that is, $5 \%$. With these uncertainties in the ${ }^{16} \mathrm{O} /{ }^{18} \mathrm{O}$ ratio and OPR, in the worst case, we may have underestimated the total water outgassing rate by $15 \%$ in the worst case or overestimated it by $11 \%$ when we used the ${ }^{16} \mathrm{O} /{ }^{18} \mathrm{O}$ measurement of Schroeder et al. (2019).

\section{Comments on specific days}

\subsection{October 2014 to 18 January 2015}

No coma maps were made between 29 July 2014 and midFebruary 2015. Only partial maps of the nucleus with part of the inner coma were made, such as the map presented in Biver et al. (2015). In order to still retrieve some information on the global outgassing, we used some observations that provided good enough sampling of the coma around the nucleus to constrain both the azimuthal and transverse extent of the outgassing, in most cases at one or two projected distances from the nucleus. 
However, the azimuthal sampling can be uneven and/or have varying S/Ns, especially for 12 and 13 October 2014. As a consequence, the determined outgassing pattern can be less precise for these dates.

\subsection{August and 1 September 2015}

On these dates, during the perihelion period, the south pole and Sun directions were closest with respect to the direction of Rosetta: a Rosetta-comet-south pole angle (colatitude angle) of $\approx 46^{\circ}-41^{\circ}$ and a Rosetta-comet-Sun angle (phase angle) of $77^{\circ}-$ $71^{\circ}$. The bulk of the outgassing was also closer to the spacecraft direction (colatitude $\approx 49^{\circ}$ ) than for the other maps. The observed lines are indeed strongly blueshifted $\left(\Delta v \approx-0.2 \mathrm{~km} \mathrm{~s}^{-1}\right)$, and the parameters of the jet were adjusted to reproduce the observed blueshifts as well as possible. In this geometry, the line intensity is much more sensitive to the modeling of the jet pattern, with an opacity of the $\mathrm{H}_{2}^{18} \mathrm{O}$ line close to 1.0 on average, and 3.0 in the most strongly blueshifted part of the line, which has strong self-absorption.

The single nadir pointing observation in these maps yields a high production rate per solid angle on the line of sight of $q_{\mathrm{H}^{16} \mathrm{O}} \approx 1.1-1.7 \times 10^{27}$ molec. $\mathrm{s}^{-1} \mathrm{sr}^{-1}$, based on the $\mathrm{H}_{2}^{18} \mathrm{O}$ and $\mathrm{H}_{2}^{17} \mathrm{O}$ lines, which is comparable to the highest value reported by Marshall et al. (2017) and is consistent with the value derived from the inverting the mapping data.

\subsection{September 2015}

The two maps were obtained in a relatively fast scanning scheme (5.4 km per spectrum). As a consequence, the projected width of the jet on the plane of the sky is likely slightly overestimated, and indeed the derived width is larger than for other dates. On the other hand, the peak intensity might be underestimated, which compensates for the overestimated width in the final computation of the production rate. The jet is also found to be mostly pointing away from the spacecraft (colatitude $\approx 130^{\circ}$ ), which also adds some uncertainty in the azimuthal retrieval of the jet width. In addition to the paucity of nadir pointing data caused by fast scanning and large comet-spacecraft distances, the low outgassing rate toward the spacecraft resulted in the absence of usable absorption lines for this period.

\subsection{September 2015}

When we average the three maps obtained on this date, all lines except for $\mathrm{CO}$ are clearly detected and the $\mathrm{S} / \mathrm{N}$ warrants retrieval of outgassing pattern information. Averaging $\mathrm{CO}$ data on large angular sectors and distance bins yields some marginal detection that also enables line inversion. These maps suggest that the bulk of CO outgassing is in the same direction as other species. Example of spectra and inversion steps are shown in Figs. 8-13. Interestingly, the width of the jet derived for methanol is line dependent (Table A.3) and is lower when the energy of the upper level of the line transition is higher (Table 1). This suggests that the higher energy levels are more populated in the center of the jet, indicating that the temperature decreases laterally from the jet axis. This lateral variation is also visible in the map of the gas temperature derived from the saturated $\mathrm{H}_{2}^{16} \mathrm{O}$ line (Fig. C.1).

\subsection{October 2015}

The four maps of 2 October 2015 were obtained at one of the largest spacecraft distances $(\approx 1200 \mathrm{~km})$ and smallest angles between the line of sight and jet axis $\left(33^{\circ}-45^{\circ}\right)$. As a consequence, the $\mathrm{H}_{2}^{18} \mathrm{O}$ line observed with line-of-sight sampling near-nucleus coma regions has a significant opacity ( $\tau$ up to 2 ), and change of a a few degrees in the direction of the jet can significantly change the line intensity and azimuthal profile. While the transverse (in the Rosetta-comet-jet plane) profile of the outgassing pattern is relatively well constrained, especially for the methanol lines, which are optically thin $(\tau<0.1)$, the distribution in the plane of the sky is not very well constrained and the derivation of $\theta_{\mathrm{az}}$ has a large uncertainty or does not converge. The line inversion suggests that the isotropic component ( $q$ in the anti-jet direction) is very small $(\approx 1 / 40$ of peak $q)$. Therefore, we assumed that its contribution is negligible when we retrieved the azimuthal width of the jet. In order to model the jet (and the opacity effects) as accurately as possible, special care was given to reproducing the observed Doppler shift of the lines ( $\Delta v=-0.19$ to $-0.37 \mathrm{~km} \mathrm{~s}^{-1}$ depending on the lines and maps).

\subsection{November 2015}

On 22 November 2015, the south pole of the comet was close to the line of sight (colatitude angle less than $30^{\circ}$ ). All lines appeared blueshifted $\left(\Delta v=-0.1\right.$ to $\left.-0.4 \mathrm{~km} \mathrm{~s}^{-1}\right)$, and the column densities on the line of sight are high, consistent with a bulk of outgassing close to the line of sight for most molecules. For this geometry it is difficult to derive the projected width of the jet on the plane of the sky (and make the deprojection) and extract the residual signal outside the jet. Hence, uncertainties on the effective solid angle of the jet and on the ratio of the isotropic (night) to the jet components of the outgassing are larger. However, the calculation of the total outgassing rate (sum of isotropic + jet outgassing) should be less strongly affected.

\subsection{December 2015}

On this day, the bulk of the outgassing clearly moved away from Rosetta (all lines were redshifted). The colatitude of the $\mathrm{CH}_{3} \mathrm{OH}$ jet $\left(140^{\circ}\right)$ was larger than for $\mathrm{H}_{2} \mathrm{O}$ and $\mathrm{NH}_{3}$ jets $\left(110^{\circ}-120^{\circ}\right)$. The derived jet widths (both in azimuth and perpendicularly) were larger than usual, so that part of the outgassing (especially for methanol) also came from the night side. Because of the large jet colatitude angles, the retrieved deprojected azimuthal width of the jet has a high uncertainty.

\section{Constraints on abundance variation with latitude obtained from nadir pointings}

\subsection{Analysis of selected nadir observations}

In order to provide further constraints on the outgassing pattern, we analyzed nadir pointings by averaging the data obtained over at least one full rotation of the nucleus. We selected observations with similar sub-spacecraft latitudes (or pole colatitude angles). The mean expansion velocity was derived from the line shapes, and the temperature profile was obtained from the (most often saturated) $\mathrm{H}_{2}^{16} \mathrm{O}$ line profile, as explained in Sect. 3.4.3. We derived the column density and production rate per unit solid angle $q$ for each line. We weight-averaged the values obtained for the different methanol lines. For water, we used the weighted average of $500 \times q\left(\mathrm{H}_{2}^{18} \mathrm{O}\right)$ and $q\left(\mathrm{H}_{2}^{16} \mathrm{O}\right)$, and $2700 \times q\left(\mathrm{H}_{2}^{17} \mathrm{O}\right)$ when the $\mathrm{S} / \mathrm{N}$ was high enough. For $\mathrm{H}_{2}^{16} \mathrm{O}$ and $\mathrm{H}_{2}^{18} \mathrm{O}, q$ was estimated from the line area integrated over channels at negative velocities and the full line area by averaging the two results according to their error bars. Imperfect modeling (acceleration 
close to the nucleus is neglected, but this is not the main source of discrepancy when testing with a radially variable expansion velocity model) and the complex structure of the outgassing pattern mean that the difference between the $q$ derived from the two parts of the line can be very high when the opacity is important $(\tau>100)$. Highly opaque lines were therefore given a low weight when we computed $q$ from these lines. When production rates became very low and $\mathrm{H}_{2}^{18} \mathrm{O}$ was weakly detected, $q_{\mathrm{H}_{2} \mathrm{O}}$ was mostly based on the main $\mathrm{H}_{2}^{16} \mathrm{O}$ line.

\subsection{Results}

The computed nadir water production rates (per solid angle) and relative abundances of $\mathrm{CH}_{3} \mathrm{OH}, \mathrm{NH}_{3}$, and $\mathrm{CO}$ are listed in Table A.5. We provide the time interval (in most cases by averaging variations over nucleus rotation), heliocentric distance, latitude of the subsolar point $\left(0^{\circ}\right.$ at equinoxes $)$ and average colatitude of the north pole $\phi_{\mathrm{N}}$, the angle between the Rosetta-comet line, and the direction of north pole (sub-spacecraft latitude = $90-\phi_{\mathrm{N}}$ ). Figure 16 shows the abundances versus sub-spacecraft latitude for selected time intervals.

Especially for $\mathrm{CO}$, for which mapping data do not give precise values for the jet width and direction, we used these measurements to obtain rough constraints on the outgassing patterns. The objective was to obtain approximate average characteristics: jet width and latitude over four periods of a few months, as listed in Table 4. The jet width and latitude of $\mathrm{H}_{2}^{16} \mathrm{O}$ were derived from those of $\mathrm{H}_{2}^{18} \mathrm{O}$ from the mapping data (Figs. 20 and 21). For each of the selected periods (Table 4) we multiplied the derived abundances versus latitude (Fig. 16) by the $\mathrm{H}_{2}^{16} \mathrm{O}$ Gaussian jet profile (meridian cut) and fit a Gaussian to the result. This gave an estimate of the width and latitude of the Gaussian jet of the considered molecule. The results are provided in Table 4: values in parentheses are weakly constrained, especially when the jet is close to the pole.

In their analysis of MIRO nadir observations, Marshall et al. (2017) derived total production rates assuming uniform outgassing in a $4 \pi$ solid angle. They multiplied the derived nadir production rates per solid angle $q_{\text {nadir }}$ by $4 \pi$. These values varied strongly with the location of the line of sight relative to the direction of outgassing. To provide more realistic values, we considered the outgassing pattern (jet width and orientation) derived previously for each molecule, and the direction of the line of sight.

When we define the ratio $\gamma=q_{\mathrm{I}} / q_{\mathrm{J}}$ of the angular production rate in the isotropic component to the peak outgassing rate per solid angle in the jet, the total outgassing rate is

$Q_{\text {total }}=\left(4 \pi \gamma+\Omega_{\text {jet }}\right) q_{\mathrm{J}}$,

with

$q_{\mathrm{J}}=\frac{q_{\text {nadir }}}{\gamma+\exp \left(-\left(\theta_{\text {off }} / \sigma_{\mathrm{J}}\right)^{2}\right)}$.

$\theta_{\text {off }}$ is the angle between the line of sight and the jet. The jet is assumed to be at the local noon meridian (phase $=0^{\circ}$ ) and originating from the determined latitude (Table 4$). \sigma_{\mathrm{J}}$ is derived from the jet width,

$\sigma_{\mathrm{J}}=\theta_{\mathrm{J}} / \sqrt{4 \ln (2)}$.

$\gamma$ can be evaluated from the results in Table A.3, but is not very well constrained. We adjusted $\gamma$ in order to minimize the fluctuations of total production rates obtained with different geometries.

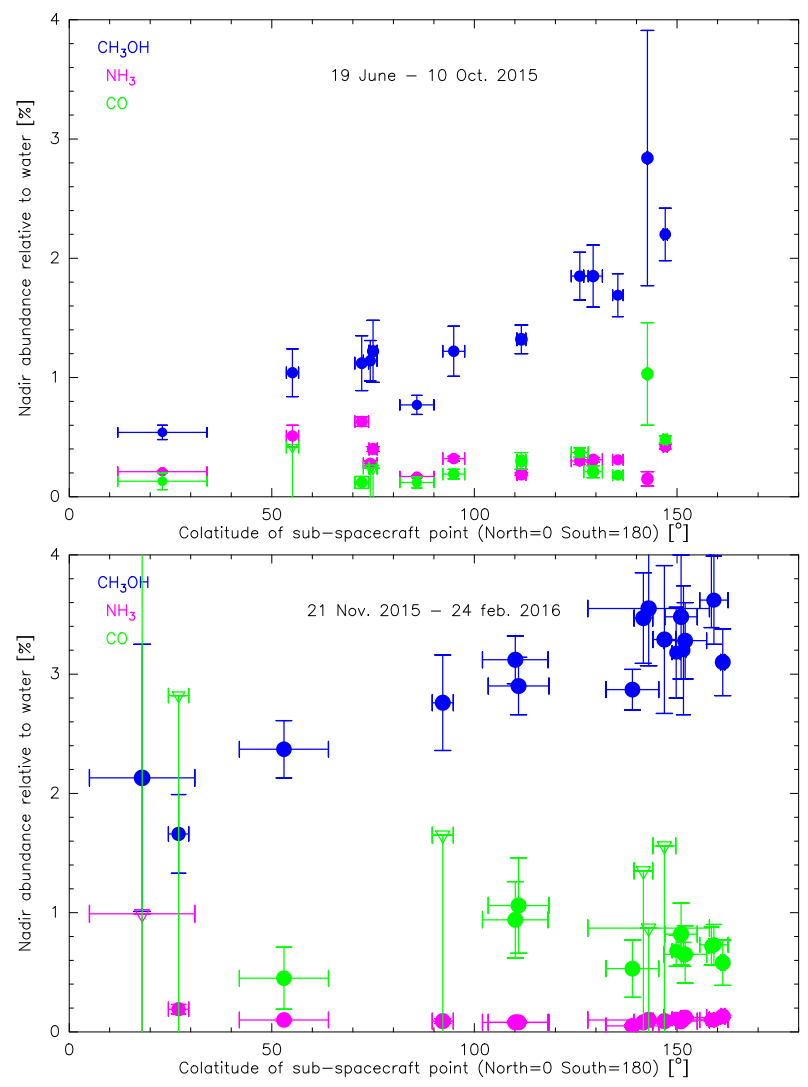

Fig. 16. Top panel: abundances of $\mathrm{CH}_{3} \mathrm{OH}, \mathrm{CO}$, and $\mathrm{NH}_{3}$ relative to water as a function of the sub-spacecraft cometocentric latitude during the nadir pointing. The selected time interval (19 June-10 October 2015) corresponds to a four-month period around perihelion. Bottom panel: same as above for the time interval of 21 November 2015-24 February 2016, four months after perihelion.

Values are in the range 0-20\%, more often 5-10\%, with $\gamma=0.07$ yielding the most satisfactory results.

The resulting ratio of $Q_{\mathrm{I}} / Q_{\text {total }}$ is then $\gamma /\left(\gamma+\Omega_{\text {jet }} / 4 \pi\right)$. However, the value of $Q_{\mathrm{I}}$ that can be deduced from this equation does not necessarily have a physical meaning, and does not imply that there is water production from the night side. This isotropic contribution is rather a mathematical way to fully describe the gas distribution around the nucleus and derive correct production rates. When the $\mathrm{S} / \mathrm{N}$ allows (e.g. in Biver et al. 2015), we always detect gas on the night side, but this may be gas released from the illuminated regions that is diffused behind. The heliocentric evolution of the total production rates derived from nadir observations is shown in Fig. 17.

\section{Distributed water sources}

The presence of a distributed water source has been proposed to explain the discrepancy of a factor of 4 between water production rates derived near perihelion from VIRTIS near-nucleus data and from ROSINA in situ data obtained at cometocentric distances $>200 \mathrm{~km}$ (Fougere et al. 2016). A discrepancy of a factor of 2.5 between the maximum water production rates is also measured by MIRO $(\sim 0.8 \times$ $10^{28}$ molec. $\left.\mathrm{s}^{-1}\right)$ and ROSINA $\left(2.04 \times 10^{28}\right.$ molec. ${ }^{-1}$; Läuter et al. 2019). The MIRO water maps, as well as the comparison of water production rates derived with different field of views, provide constraints on the existence of distributed water sources, 
Table 4. Molecular jet characteristics retrieved from nadir pointing measurements.

\begin{tabular}{|c|c|c|c|c|c|c|c|c|c|c|}
\hline \multirow{2}{*}{$\begin{array}{l}\text { Date }(\mathrm{UT}) \\
\text { yyyy/mm/dd-mm/dd }\end{array}$} & \multirow{2}{*}{$\begin{array}{c}r_{\mathrm{h}} \\
(\mathrm{AU}) \\
\end{array}$} & \multirow{2}{*}{$\begin{array}{c}\text { Subsolar latitude } \\
\left(^{\circ}\right)\end{array}$} & \multicolumn{2}{|c|}{$\mathrm{H}_{2} \mathrm{O}$ "jet" (a) } & \multicolumn{2}{|c|}{$\mathrm{CH}_{3} \mathrm{OH}$ “jet" } & \multicolumn{2}{|c|}{$\mathrm{NH}_{3}$ "jet" } & \multicolumn{2}{|c|}{ CO "jet" } \\
\hline & & & $F W H M$ & Latitude & $F W H M$ & Latitude & $F W H M$ & Latitude & $F W H M$ & Latitude \\
\hline 2015/05/16 & 1.63 & -2.2 & $130^{\circ}$ & $-9^{\circ}$ & & & & & & \\
\hline $2015 / 04 / 22-05 / 31$ & 1.66 & $-1.3 \pm 8.0$ & & & $136 \pm 9^{\circ}$ & $-30 \pm 3^{\circ}$ & $163 \pm 15^{\circ}$ & $-45 \pm 12^{\circ}$ & $\sim 63^{\circ}$ & $\sim-30^{\circ}$ \\
\hline $2015 / 07 / 13-09 / 15$ & 1.27 & $-42 \pm 10$ & $122^{\circ}$ & $-74^{\circ}$ & & & & & & \\
\hline $2015 / 06 / 19-10 / 10$ & 1.34 & $-40 \pm 16$ & & & $111 \pm 17^{\circ}$ & $(-85)^{\circ}$ & $116 \pm 12^{\circ}$ & $-73 \pm 18^{\circ}$ & $101 \pm 10^{\circ}$ & $(-85)^{\circ}$ \\
\hline $2015 / 11 / 26-13 / 14$ & 1.94 & $-20 \pm 7$ & $123^{\circ}$ & $-52^{\circ}$ & & & & & & \\
\hline $2015 / 11 / 21-14 / 24$ & 2.07 & $-16 \pm 12$ & & & $114 \pm 5^{\circ}$ & $-55 \pm 4^{\circ}$ & $\sim 123^{\circ}$ & $(-50)^{\circ}$ & $89 \pm 18^{\circ}$ & $-38 \pm 9^{\circ}$ \\
\hline $2016 / 04 / 22-05 / 16$ & 2.93 & $+5.4 \pm 1.5$ & $122^{\circ}$ & $+0^{\circ}$ & & & & & & \\
\hline $2016 / 05 / 02-06 / 19$ & 3.13 & $+9 \pm 3$ & & & $120 \pm 30^{\circ}$ & $+9 \pm 11^{\circ}$ & & & & \\
\hline
\end{tabular}

Notes. ${ }^{(a)}$ Average from maps (Table A.3) used to infer values for other molecules.

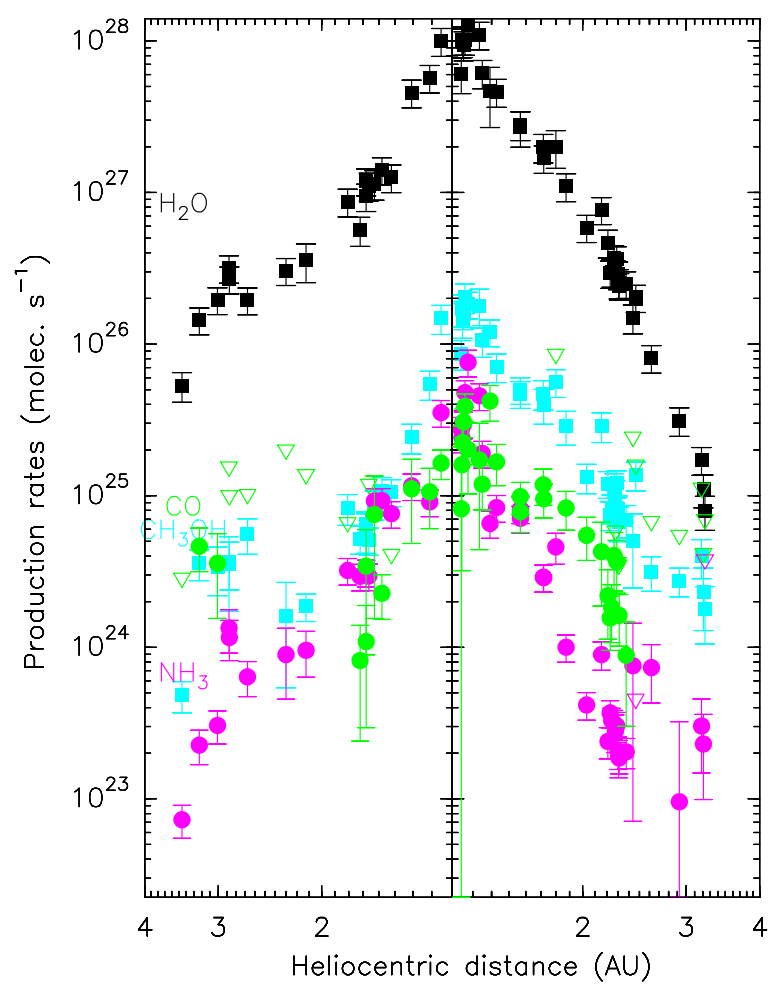

Fig. 17. Total production rates of water (black, assumed to be $500 \times Q_{\mathrm{H}_{2}^{18} \mathrm{O}}$ ) methanol (cyan), carbon monoxide (green), and ammonia (magenta) as a function of heliocentric distance, based on nadir integrations. Empty down-pointing triangles correspond to $3 \sigma$ upper limits. The horizontal scale is the heliocentric distance (pre-perihelion on the left, and post-perihelion on the right).

such as the sublimation from icy grains, or the decomposition of water dimers and clusters in cometary atmospheres (Crifo \& Slanina 1991).

When the maps are sufficiently extended, we can investigate the evolution of the total water production rate as a function of the projected distance $(\rho)$ from the nucleus. If water were partly released by some distributed source, we would expect the production rate to increase with increasing $\rho$.

We have computed production rates for a set of circular annuli $(n)$ covering each $\rho=2.5 n$ to $2.5(n+1) \mathrm{km}$ using the same method as for the global map (i.e. deriving angular production rates and the jet FWHM in each annulus). This method presents some biases that are due to (i) the scanning scheme, which introduces significant smearing for the innermost annuli, and (ii) variations of the temperature and gas velocity with distance to the nucleus, which were taken into account as well as possible, however.

Figure 18 shows two of the main significant results. Within error bars, no variation is observed for the derived production rates over the first $20 \mathrm{~km}$ from the nucleus: the variation is $+6 \pm 5 \%$ per $10 \mathrm{~km}$ on 20 August and $+7 \pm 9 \%$ per $10 \mathrm{~km}$ on 8 September 2015. A simulation gives an increase of $+17 \%$ per $10 \mathrm{~km}$ (in projected distance) in apparent production for a real increase in production of $+10 \%$ per $10 \mathrm{~km}$ (in radial distance). Based on this, we can conclude that the contribution of icy grains to the total water content at $20 \mathrm{~km}$ from the nucleus is less than $15 \%$ ( $3 \sigma$ upper limit).

Odin, a submillimeter satellite in Earth orbit (Nordh et al. 2003; Frisk et al. 2003), also observed the $\mathrm{H}_{2}^{16} \mathrm{O}$ line at $556.936 \mathrm{GHz}$ in comet $67 \mathrm{P}$ on $9.47-12.10$ November 2015 at a geocentric distance of $1.79 \mathrm{AU}$. Owing to the 2.1' Odin beam width, the field of view was $165000 \mathrm{~km}$. The line was not detected, with a $3 \sigma$ upper limit to the line area of $0.057 \mathrm{~K} \mathrm{~km} \mathrm{~s}^{-1}$. Adopting excitation parameters similar to those used for analyzing the MIRO observations, we derive an upper limit for the water production rate $Q_{\mathrm{H}_{2} \mathrm{O}}<3.3 \times 10^{27}$ molec. $\mathrm{s}^{-1}$ (Snodgrass et al. 2016). The total water production rate estimated from MIRO measurements at the same time (11.5 November) is $2.2 \pm$ $0.1 \times 10^{27}$ molec. $\mathrm{s}^{-1}$. Because the average cometocentric distance sampled by MIRO is on the order of $15 \mathrm{~km}$, the comparison between the two measurements indicates that if there was significant water production from a distributed source beyond $20 \mathrm{~km}$, then its production rate did not exceed $50 \%$ of the value measured by MIRO. Altogether, our investigation of the contribution of sublimating icy grains to the total water content at $20 \mathrm{~km}$ from the nucleus and our comparison with the Odin data suggests that the discrepancy of a factor 2.5 between the production rates derived from MIRO and ROSINA measurements cannot be explained by sublimating icy grains.

\section{9. $\mathrm{H}_{2}^{17} \mathrm{O} / \mathrm{H}_{2}^{18} \mathrm{O}$ ratio}

$\mathrm{H}_{2}^{17} \mathrm{O}$ is detected in MIRO spectra (e.g. Fig. E.1). This is the first spectroscopic detection of cometary $\mathrm{H}_{2}^{17} \mathrm{O}$. Deriving the oxygen isotopic ratios from the MIRO data is a complex task. The opacities of the $\mathrm{H}_{2}^{16} \mathrm{O}, \mathrm{H}_{2}^{18} \mathrm{O}$ and $\mathrm{H}_{2}^{17} \mathrm{O}$ lines are very different and the dynamical range of the MIRO spectra is limited, therefore 

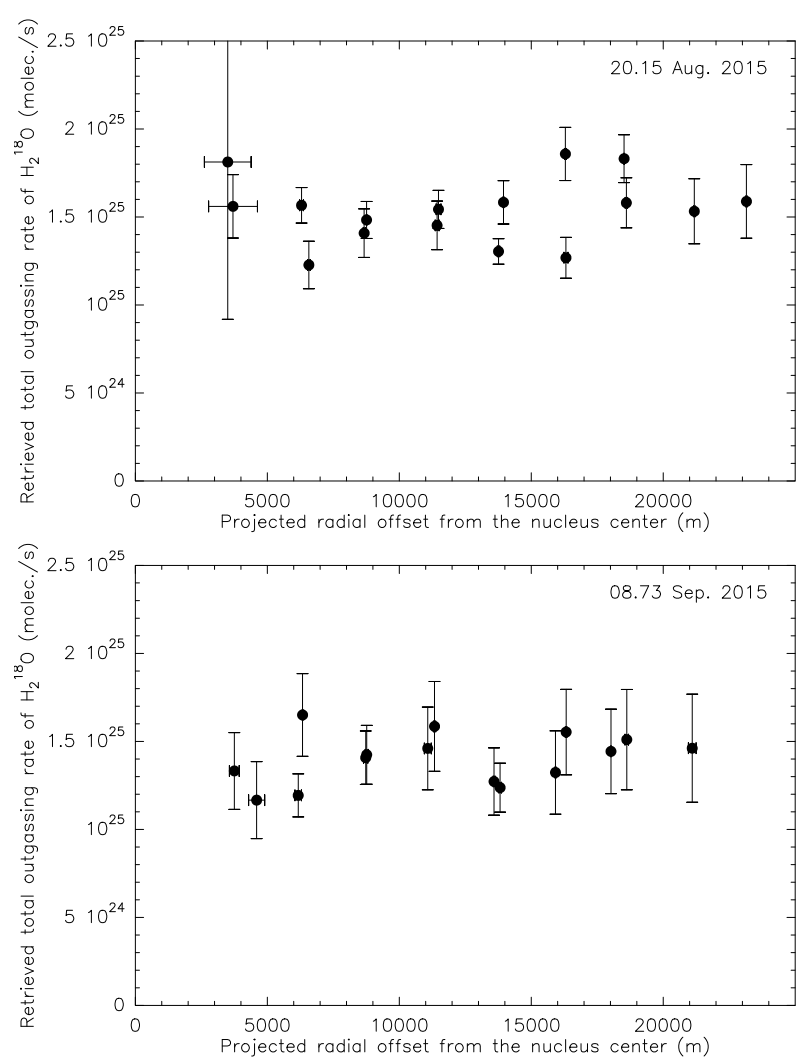

Fig. 18. Top panel: retrieved total water production rate as a function of the projected distance $\rho$ from the center of the nucleus, based on selected rings in the two maps of $\mathrm{H}_{2}^{18} \mathrm{O}$ on 20 August 2015. Bottom panel: same for the mapping data of 8 September 2015.

we cannot compare two lines in a linear regime. A modeling of the line profiles using very accurate velocity, temperature, and density profiles is necessary to derive precise isotopic ratios. In addition, the $\mathrm{H}_{2}^{17} \mathrm{O}$ line occupies most of the spectral window, which makes an accurate determination of the baseline difficult.

Here we computed the $\mathrm{H}_{2}^{17} \mathrm{O} / \mathrm{H}_{2}^{18} \mathrm{O}$ ratio by forming a ratio of the production rates (or production rates per solid angle for nadir observations) derived for $\mathrm{H}_{2}^{18} \mathrm{O}$ and $\mathrm{H}_{2}^{17} \mathrm{O}$ (Fig. 19). There is significant scatter in the derived $\mathrm{H}_{2}^{18} \mathrm{O} / \mathrm{H}_{2}^{17} \mathrm{O}$ in ratios, but no significant trend with time (hence with production rate or opacity) is observed. Some higher values are measured in nadir pointing alone in February 2016, but the detection of $\mathrm{H}_{2}^{17} \mathrm{O}$ is marginal for these observations, and retrievals are very sensitive to the baseline removal. The average value using all measurements is $\mathrm{H}_{2}^{18} \mathrm{O} / \mathrm{H}_{2}^{17} \mathrm{O}=5.45 \pm 1.3$. A value of $5.6 \pm 0.8$ is derived when we only consider the jet production rates, which have lower uncertainties (squares in Fig. 19, with values listed in Table A.4). This is compatible with the telluric value (Vienna Standard Mean Ocean Water VSMOW = 5.28) and the ROSINA measurement performed at the beginning of the mission $(4.9 \pm 1.2$ from Altwegg et al. 2015).

\section{Long-term evolution of the outgassing}

The analysis of the maps presented here provides us with information on the long-term evolution of the outgassing pattern and production rate of the various molecules observed with MIRO. Because the maps were acquired over a few hours, rotational variations are to some extent averaged out. Comet 67P exhibited numerous dusty outbursts, especially during the perihelion

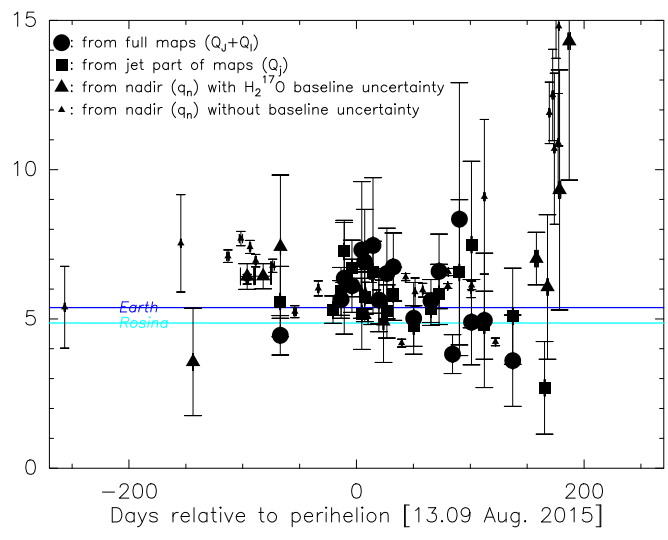

Fig. 19. Production rates ratio of $\mathrm{H}_{2}^{18} \mathrm{O}$ to $\mathrm{H}_{2}^{17} \mathrm{O}$ as a function of time. The dots refer to total production rates based on maps. Squares refer only to the ratio of production rates in the jet, which have smaller error bars (data from Tables A.3 and A.4). Triangles refer to nadir measurements of the production rate per unit solid angle, including uncertainties due to the $\mathrm{H}_{2}^{17} \mathrm{O}$ baseline when specified (larger symbols). The Earth (VSMOW) value and the value derived from the ROSINA experiment (Altwegg et al. 2015) are shown for comparison.

period (Vincent et al. 2016). However, there is little evidence for significant gas enhancement during outbursts in MIRO data (Marshall et al. 2017; Grün et al. 2016).

\subsection{Outgassing pattern}

Figures 20 and 21 show the time evolution of the source region and width of the jet for the different molecules. The seasonal evolution is clearly visible in these two plots: before the first equinox (10 May 2015, 95 days before perihelion), the outgassing originates from the northern hemisphere $\left(50^{\circ} \mathrm{N}\right)$ and is concentrated in a narrow jet $\left(F W H M \sim 70^{\circ}-90^{\circ}\right.$, Fig. 21$)$. The neck region of $67 \mathrm{P}$ is the main source of outgassing during this period, as also observed by other Rosetta instruments (e.g. Migliorini et al. 2016, for VIRTIS). After the first equinox, the activity of 67P moved rapidly to the southern hemisphere. The outgassing also spread over a wider part of the nucleus. The width of the jet reached its maximum at equinox, on the order of $140^{\circ}$; in some maps, two jet components were required to best fit the spatial distribution (e.g. for methanol on 15 May and 24 June 2015, Table A.3).

During the perihelion period, when the gaseous activity was high, the outgassing originated essentially from the southernmost regions, even farther south than the subsolar point, and the jet width was about $110^{\circ}$. After the second equinox (21 March 2016, 220 days after perihelion), the water outgassing returned to the northern hemisphere (Fig. 20), while methanol and ammonia continued to be mainly released from southern regions. In the last MIRO maps, obtained later than 250 days after perihelion, all species originated from the northern hemisphere. Compared with water and methanol jets, the latitude of the ammonia jet is in general closer to the subsolar latitude. On the other hand, methanol always comes from farther south than $\mathrm{H}_{2} \mathrm{O}$ and $\mathrm{NH}_{3}$. Compared with ROSINA results, the seasonal evolution of the outgassing of $\mathrm{CH}_{3} \mathrm{OH}$ follows that of $\mathrm{CO}_{2}$ (Läuter et al. 2019). In Fig. 20, using a degraded version of the nucleus shape model SPG SHAP7 (Preusker et al. 2017) with 125000 facets, we have also plotted the expected average latitude of the surfaces that receive at least $95 \%$ of the maximum solar flux over a 3 days period. As can be anticipated this gives a closer match than 


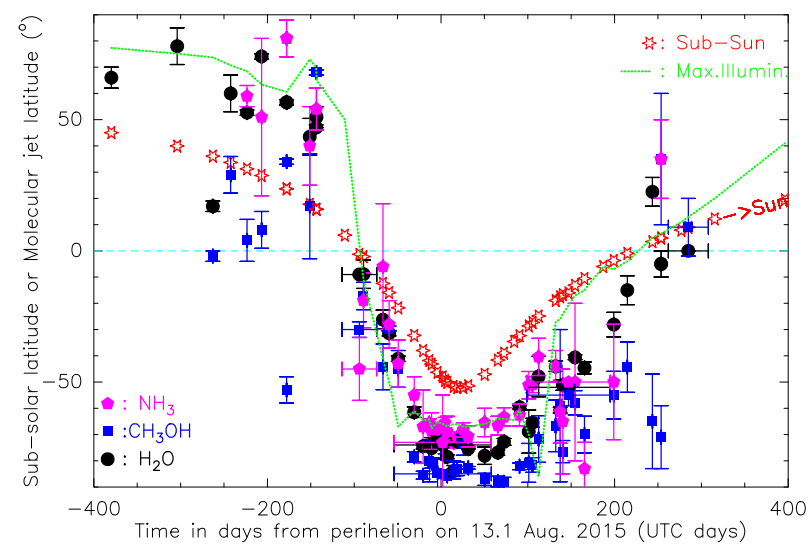

Fig. 20. Evolution of the mean latitude of the bulk of outgassing (jet) water, methanol, and ammonia from July 2014 to September 2016. The red stars show the latitude of the subsolar point, which crosses the equator at the comet equinoxes, 95 days before perihelion and 220 days after. The green dotted line corresponds to the average latitude of the facets that receive the maximum solar flux.

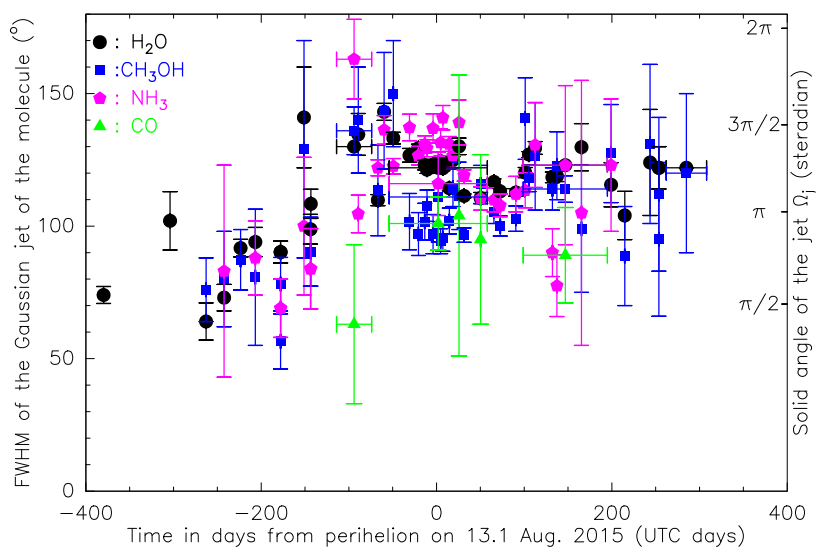

Fig. 21. Evolution of the mean angular extent of the bulk of outgassing (jet FWHM) water, methanol, and ammonia from July 2014 to September 2016. Representative values of the extent of the solid angle of the jet (non-linear scale) are given on the right.

the sub-solar latitude to the evolution of the latitude of peak of outgassing, possibly with some delay post-perihelion caused by thermal inertia.

\subsection{Evolution of outgassing and relative abundances in the coma}

Figure 22 shows the production rates as a function of heliocentric distance and time, derived from the maps. Results from nadir measurements are presented in Fig. 17. CO production rates derived from nadir measurements have a better $\mathrm{S} / \mathrm{N}$ ratio, but are marginally lower than those deduced from mapping data. The test on the 30 August 2015 shows that the nadir-derived CO production rate is more sensitive to the temperature profile than the rate derived from the maps: if we increased the terminal $\mathrm{CO}$ temperature by a factor 2 (compared to the adopted profile for other molecules), the retrieved $\mathrm{CO}$ production rate would increase by the same factor and the $\mathrm{CO}$ abundances derived from maps and nadir observations would be reconciled. On the other hand, nadir-derived production rates are about $30 \%$ higher for the other molecules. The nadir measurements are more uncertain because they rely on our Gaussian jet modeling, which is

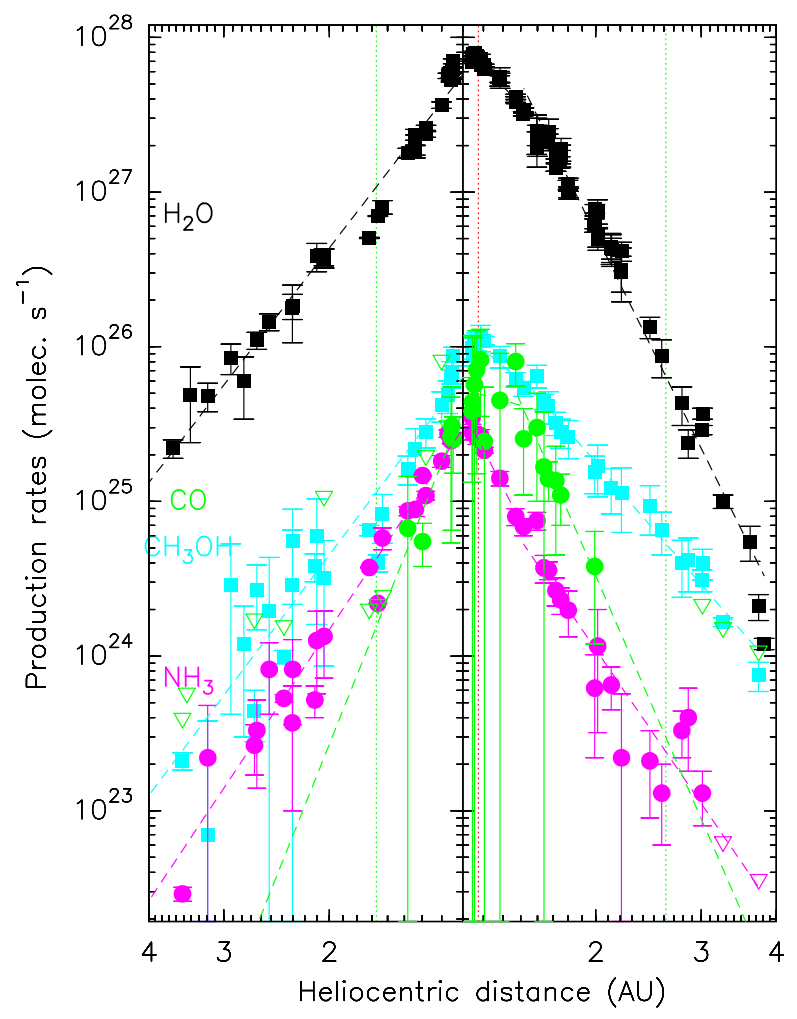

Fig. 22. Heliocentric evolution of the total production rates of water (black, assumed to be $500 \times Q_{\mathrm{H}_{2}^{18} \mathrm{O}}$ ), methanol (cyan), carbon monoxide (green), and ammonia (magenta) from July 2014 to September 2016, based on mapping data. The horizontal scale is the heliocentric distance (pre-perihelion on the left, and post-perihelion on the right). Empty down-pointing triangles correspond to $3 \sigma$ upper limits. Dotted lines are power-law fits to the heliocentric evolution (see Table 5).

too simplistic to describe the density distribution along specific line of sights.

We derived power-law fits $\left(Q\left(r_{\mathrm{h}}\right)=Q_{0} r_{\mathrm{h}}^{\beta}\right)$ for the heliocentric evolution of the production rates, which are shown in Fig. 22. Three periods were considered: pre-perihelion, post-perihelion below 1.52 AU, and post-perihelion beyond 1.52 AU. The fit parameters are provided in Table 5. The heliocentric variation in production rates is much steeper $\left(\propto r_{\mathrm{h}}^{-5}\right.$ to $\left.r_{\mathrm{h}}^{-8}\right)$ than observed for long-period comets such as C/1995 O1 (Hale-Bopp; Biver et al. 2002). This steep variation is due to the strong activity of the southern hemisphere during summer and points to strong seasonal effects. The heliocentric evolution of the outgassing rates of $\mathrm{H}_{2} \mathrm{O}, \mathrm{CO}_{2}, \mathrm{CO}$, and other species has been studied by Hansen et al. (2016), Gasc et al. (2017), Läuter et al. (2019), using ROSINA data. MIRO results are in line with the results of Läuter et al. (2019), who also found steep variations for CO and $\mathrm{CO}_{2}$ (to which $\mathrm{CH}_{3} \mathrm{OH}$ seems to be linked because they have similar outgassing patterns), but with a lower total abundance of $\mathrm{CO}(\approx 0.6 \%$ versus $2 \%$ post-perihelion before equinox $)$ for MIRO. The maximum outgassing rates are measured at the end of August and beginning of September 2015, that is, about three weeks after perihelion. This is consistent with ROSINA measurements (Hansen et al. 2016).

Figure 23 shows the evolution of the production rate ratios of $\mathrm{CH}_{3} \mathrm{OH}, \mathrm{NH}_{3}$, and $\mathrm{CO}$ relative to the water production rate (hereafter called abundances because they refer to abundances in the coma). Mapping and nadir measurements are both plotted and show consistent results. The abundances display clear 
N. Biver et al.: Monitoring of comet 67P with Rosetta/MIRO

Table 5. Power-law fit $\beta$ to the production rates $\left(Q_{0} r_{\mathrm{h}}^{\beta}\right)$ based on mapping data.

\begin{tabular}{lcccc}
\hline \hline Molecule & $\mathrm{H}_{2} \mathrm{O}$ & $\mathrm{CH}_{3} \mathrm{OH}$ & $\mathrm{NH}_{3}$ & $\mathrm{CO}$ \\
\hline \multicolumn{5}{c}{ Data based on analysis of mapping data } \\
\hline Pre-perihelion & $-5.0 \pm 0.1$ & $-5.2 \pm 0.5$ & $-5.8 \pm 0.3$ & $-9.7 \pm 2.6$ \\
Post-perihelion $r_{\mathrm{h}}<1.52 \mathrm{AU}$ & $-4.1 \pm 0.2$ & $-3.2 \pm 0.7$ & $-7.6 \pm 0.4$ & $-1.1 \pm 2.6$ \\
Post-perihelion $r_{\mathrm{h}}>1.52 \mathrm{AU}$ & $-7.9 \pm 0.2$ & $-4.5 \pm 0.2$ & $-5.7 \pm 0.7$ & $-8.9 \pm 1.3$ \\
\hline \multicolumn{5}{c}{ Data based on analysis of nadir pointings } \\
\hline Pre-perihelion $r_{\mathrm{h}}<2.8 \mathrm{AU}$ & $-5.0 \pm 0.5$ & $-6.8 \pm 0.8$ & $-5.0 \pm 0.6$ & $-7.6 \pm 2.3$ \\
Post-perihelion $r_{\mathrm{h}}<1.7 \mathrm{AU}$ & $-5.3 \pm 0.7$ & $-4.5 \pm 0.9$ & $-7.8 \pm 1.5$ & $-2.4 \pm 1.5$ \\
Post-perihelion $r_{\mathrm{h}}>1.7 \mathrm{AU}$ & $-8.4 \pm 0.4$ & $-4.9 \pm 0.5$ & $-4.2 \pm 1.0$ & $-6.3 \pm 1.2$ \\
\hline
\end{tabular}

time variations. In particular, after perihelion, the methanol and $\mathrm{CO}$ abundances increase with increasing heliocentric distance, while ammonia becomes less abundant in the coma. As discussed in Sect. 10.1, after perihelion, $\mathrm{CH}_{3} \mathrm{OH}$ mainly outgassed from the southernmost regions, while water production progressively moved from southern to northern regions. The increase in methanol abundance in the coma after perihelion can then be explained by the sustained activity of the southern hemisphere, whereas the $\mathrm{H}_{2} \mathrm{O}$ activity decreased more rapidly. The similar behavior that is observed for $\mathrm{CO}$ suggests that the southern hemisphere was also overproductive in carbon monoxide. This is consistent with ROSINA measurements, which showed that whereas the source locations of $\mathrm{H}_{2} \mathrm{O}$ followed the subsolar latitude, $\mathrm{CO}_{2}$ sources were mainly located in southern areas during the whole mission, with $\mathrm{CO}$ correlating with $\mathrm{CO}_{2}$ after perihelion (Läuter et al. 2019). Overall, $\mathrm{NH}_{3}$ correlates with $\mathrm{H}_{2} \mathrm{O}$ in the source region (Fig. 20). This confirms results obtained from ROSINA (Gasc et al. 2017) and suggests that $\mathrm{NH}_{3}$ may be trapped into water ice (e.g. as a trapped species into water amorphous ice, or in the form of a hydrate). The continuous decrease in $\mathrm{NH}_{3}$ abundance is surprising and may suggest that the relative abundance of $\mathrm{NH}_{3}$ embedded in water ice is higher in the southern than in the northern hemisphere. The northern hemisphere is covered by smooth layers of back-fall ice-rich material originating from the southern hemisphere (Keller et al. 2017). This would mean that the chunks that fall back lost some of their volatiles during their flight in the coma, as suggested by Fulle et al. (2019).

Comparing the outgassing patterns and heliocentric trends followed by several species (not including $\mathrm{CH}_{3} \mathrm{OH}$ ), Gasc et al. (2017) did not find any correlation between outgassing properties and the sublimation temperature of the corresponding pure ices. This result is confirmed by the MIRO results. $\mathrm{NH}_{3}$ and $\mathrm{CH}_{3} \mathrm{OH}$ ices have about the same sublimation temperature ( 78 and $99 \mathrm{~K}$, respectively; Yamamoto 1985) but behave differently. Gasc et al. (2017) proposed that minor species could be partly embedded in $\mathrm{CO}_{2}$ and partly in $\mathrm{H}_{2} \mathrm{O}$ ices. Their different power laws with heliocentric distance would therefore be indicative for the ratio between how much is embedded in $\mathrm{CO}_{2}$ and how much is embedded in water.

It is interesting to compare MIRO-derived molecular abundances to those deduced from other instruments. Le Roy et al. (2015) derived from ROSINA data $\mathrm{CH}_{3} \mathrm{OH} / \mathrm{H}_{2} \mathrm{O}$ abundance ratios in the range $0.31-0.55 \%$ at $3.2 \mathrm{AU}$ inbound, where the lower and higher values correspond to the summer (north) and winter (south) hemispheres, respectively. This is a factor of 2 lower than the mean MIRO value for 2.9-3.46 AU inbound. Their derived $\mathrm{NH}_{3} / \mathrm{H}_{2} \mathrm{O}$ ratio of $0.06-0.15 \%$ at $3.2 \mathrm{AU}$ pre-perihelion is also somewhat lower than our value $(0.2 \%)$. The discrepancy is still higher post-perihelion. Gasc et al. (2017) reported a $\mathrm{NH}_{3} / \mathrm{H}_{2} \mathrm{O}$ value as low as $0.04 \%$ for $2.0-2.7 \mathrm{AU}$ postperihelion, whereas the MIRO average value for this period is $0.1 \%$. The $\mathrm{CO} / \mathrm{H}_{2} \mathrm{O}$ ratio derived from ROSINA instruments ranges from $\sim 1$ to $\sim 50 \%$, with strong local and temporal variations (Le Roy et al. 2015; Fougere et al. 2016; Hoang et al. 2017; Gasc et al. 2017). A steep decrease from 3.6 to 2.6 AU inbound was observed before it stabilized at a value of about $5 \%$ (Fougere et al. 2016). Values measured at heliocentric distances larger than 3.1 AU, inbound and outbound, reach 50\%. Läuter et al. (2019) provided the integrated production of water and $\mathrm{CO}$ deduced from ROSINA data, and found a bulk $\mathrm{CO} / \mathrm{H}_{2} \mathrm{O}$ abundance of $2.2 \pm 0.9 \%$. As shown in Fig. 23, the $\mathrm{CO} / \mathrm{H}_{2} \mathrm{O}$ ratios measured by MIRO $\left(r_{\mathrm{h}}<2.4 \mathrm{AU}\right)$ are all below $1 \%$. Measurements with the Alice instrument shortly after perihelion lead to $\mathrm{CO} / \mathrm{H}_{2} \mathrm{O} \sim 0.5 \%$ (Feldman et al. 2018), in agreement with MIRO determinations. A low $(<1 \%) \mathrm{CO} / \mathrm{H}_{2} \mathrm{O}$ abundance near perihelion is also consistent with the upper limit measured from infrared spectra obtained by VIRTIS (Bockelée-Morvan et al. 2016). In summary, there are significant discrepancies between in situ mass-spectrometer data and remote-sensing spectroscopic data for the abundance ratios.

It is also interesting to compare the abundances measured in comet 67P to those measured in other comets. This was investigated by Le Roy et al. (2015) using the ROSINA data acquired at 3.2 AU inbound. We here used MIRO data acquired near perihelion because most comets were observed near their perihelion. Bockelée-Morvan \& Biver (2017) presented histograms of abundances based on radio measurements of 46 comets. 67P appears to have an ordinary methanol abundance, and does not belong to the methanol-rich group of comets that exhibit abundances relative to water in the range $3-6 \%$. The $\mathrm{NH}_{3}$ abundance of $67 \mathrm{P}$, typically $0.4 \%$ at perihelion, is in the range of values observed for other comets: $0.2-0.7 \%$ from radio measurements (BockeléeMorvan \& Biver 2017) and 0.1-1.7\% from infrared data (Dello Russo et al. 2016). Comets observed near $1 \mathrm{AU}$ from the Sun display $\mathrm{CO} / \mathrm{H}_{2} \mathrm{O}$ production rate ratios in the range $0.2-23 \%$ (Bockelée-Morvan \& Biver 2017). Comet 67P value is at the low end of this range, as are other Jupiter-family comets. This possibly results from a stratification in the ice composition of the subsurface layers of 67P caused by its multiple passages close to the Sun, with the most volatile species only residing in very deep layers.

\subsection{Bulk abundances and mass losses}

We have computed the cumulative mass loss of volatile species during the 2015 apparition of 67P. The results for water and 

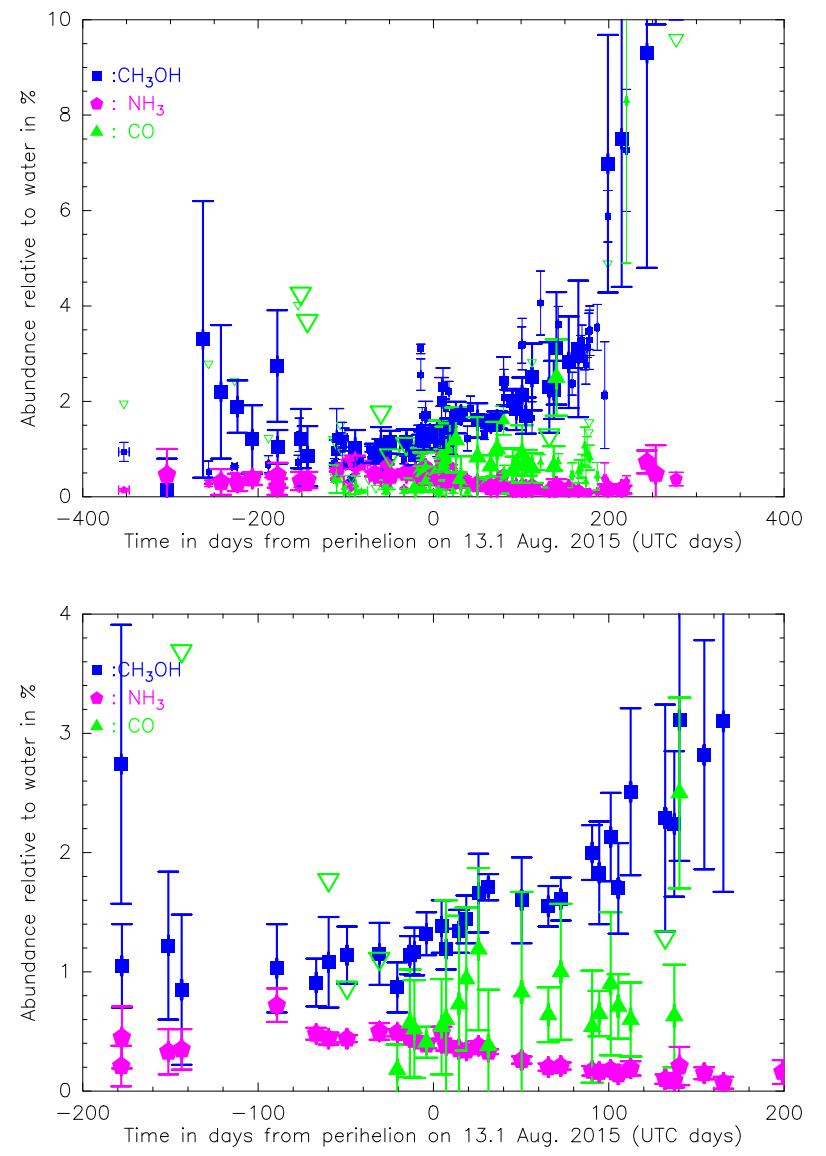

Fig. 23. Evolution of the abundances relative to water of methanol, ammonia, and CO in the coma from July 2014 to September 2016. The larger symbols are based on the global outgassing rates derived from mapping data. Additional more local measurements from nadir or single lines of sight are shown by smaller symbols in the upper panel. Empty down-pointing triangles correspond to $3 \sigma$ upper limits. Bottom panel: zoom over \pm 200 days around perihelion.

the other molecules observed by MIRO, obtained using linear interpolation between each point, are shown in Figs. 24 and 25, respectively. The total mass losses are listed in Table 6 . Because CO data are lacking beyond 140 days post-perihelion, we assumed that the $\mathrm{CO}$ production followed the trend of $\mathrm{CH}_{3} \mathrm{OH}$ in that period. Results obtained using the derived power laws for the heliocentric dependence of the production rates provide similar results (Table 6). Overall, 95\% of the volatile mass was lost between the equinoxes, when the southern hemisphere was exposed to the Sun. Ratioing the mass-loss rates to that of water, the relative mass-loss rates are $1.5 \%, 0.34 \%$, and $0.6 \%$ for $\mathrm{CH}_{3} \mathrm{OH}, \mathrm{NH}_{3}$, and $\mathrm{CO}$, respectively, with a large uncertainty for $\mathrm{CO}$ (Table 6). For $\mathrm{CO}$, we adopt a mean abundance of $0.6 \pm 0.2 \%$ as a compromise between mapping and nadir measurements.

The derived total water loss derived from the mapping data is $2.4 \pm 0.1 \times 10^{9} \mathrm{~kg}$ (Table 6), which is a factor of 2-3 lower than the values derived from ROSINA data: $4.8 \pm 1.5 \times 10^{9} \mathrm{~kg}$ (Läuter et al. 2019) and $6.9 \times 10^{9} \mathrm{~kg}$ (Hansen et al. 2016). The values derived from the MIRO nadir data are $17-35 \%$ higher than those obtained from mapping data and consistent with the mappingderived value for the power-law fits. The nadir-integrated mass loss is probably slightly biased upward by the value of 5 September, but the retrieved abundances are fully consistent within the error bars. We note that Marshall et al. (2017) derived

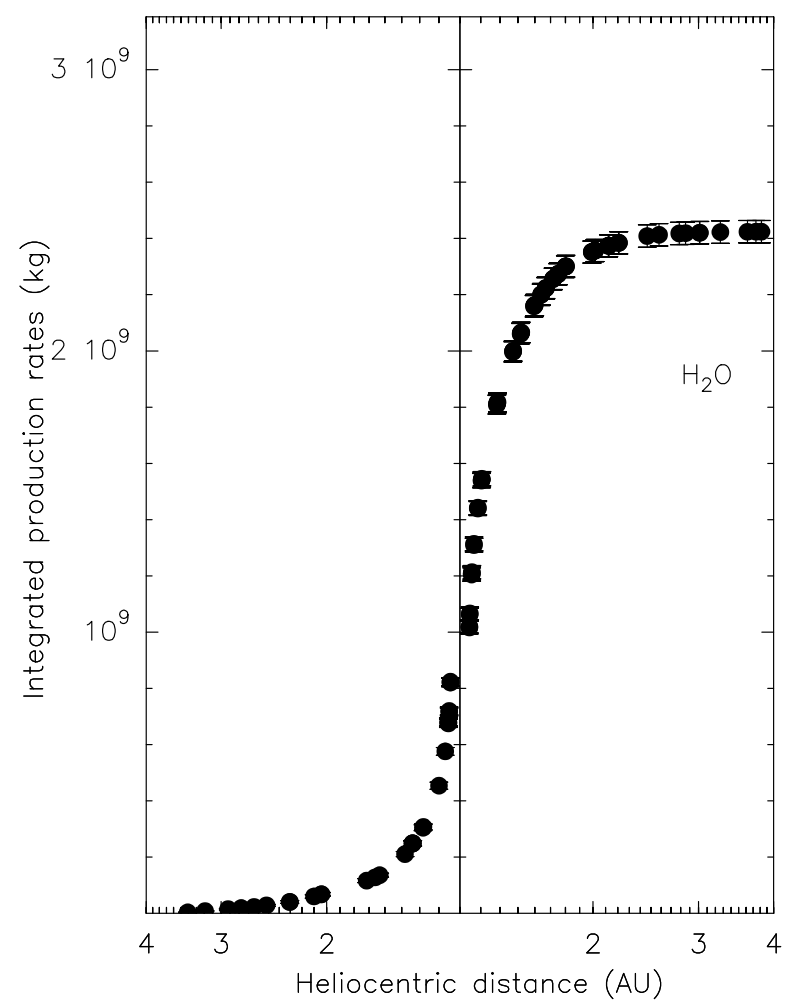

Fig. 24. Cumulative water mass-loss rate of comet 67P from July 2014 to September 2016. About $40 \%$ more mass is lost post-perihelion than pre-perihelion ( $\sim 1.4$ vs. 1 million tons), but mid-point (50\% cumulative loss) is close to the solstice (vertical red dotted line). Ninety-eight percent of the mass is cumulatively lost within \pm 200 days from perihelion, and $95 \%$ between the two equinoxes (vertical blue dotted lines). The total water loss is $2.42 \pm 0.04 \times 10^{9} \mathrm{~kg}$.

a value of $2.4 \times 10^{9} \mathrm{~kg}$ from his analysis of the nadir MIRO data, which is consistent with ours.

In order to evaluate the total mass loss from ice sublimation, we have to consider other molecules, which were not observed by MIRO but are significant contributors to the gas activity: $\mathrm{CO}_{2}, \mathrm{O}_{2}$, sulfur compounds and hydrocarbons. In VIRTIS data (Bockelée-Morvan et al. 2015a, 2016) and ROSINA measurements (Gasc et al. 2017), the $\mathrm{CO}_{2}$ abundance showed a somewhat similar behavior to methanol, but the abundance was about 16 times higher around perihelion. Calmonte et al. (2016) have estimated that the elemental $\mathrm{S} / \mathrm{O}$ ratio (in numbers) in the gas phase is on the order of 0.015 . For $\mathrm{O}_{2}$, we used the average abundance relative to water of 0.02 (in numbers) provided by Läuter et al. (2019). Hydrocarbons and other molecules do not significantly contribute to the mass budget (i.e. by less than a few percent) because of their low abundances (and low molecular masses for the light hydrocarbons). Summing contributions, we find a total ice loss of $4.18 \pm 0.18 \times 10^{9} \mathrm{~kg}$ (the uncertainty related to uncertainties in the OPR and ${ }^{16} \mathrm{O} /{ }^{18} \mathrm{O}$ is $11 \%$ at most and is not considered here). A $28 \%$ higher value of $5.36 \pm$ $0.35 \times 10^{9} \mathrm{~kg}$ is derived using nadir data and assuming an OPR of $2.5-3.0$ and ${ }^{16} \mathrm{O} /{ }^{18} \mathrm{O}=445 \pm 35$, which is slightly more consistent with ROSINA in situ measurements (Hansen et al. 2016; Läuter et al. 2019). However, as discussed above, we are less confident with this result because the modeling approach is limited.

The net mass loss of the nucleus of comet 67P between September 2014 and June 2016 based on Radio Science Investigations (RSI) of the gravity field (Pätzold et al. 2019) is $10.5 \pm$ $3.4 \times 10^{9} \mathrm{~kg}$, that is, $0.1 \%$ of the nucleus mass, and about one 


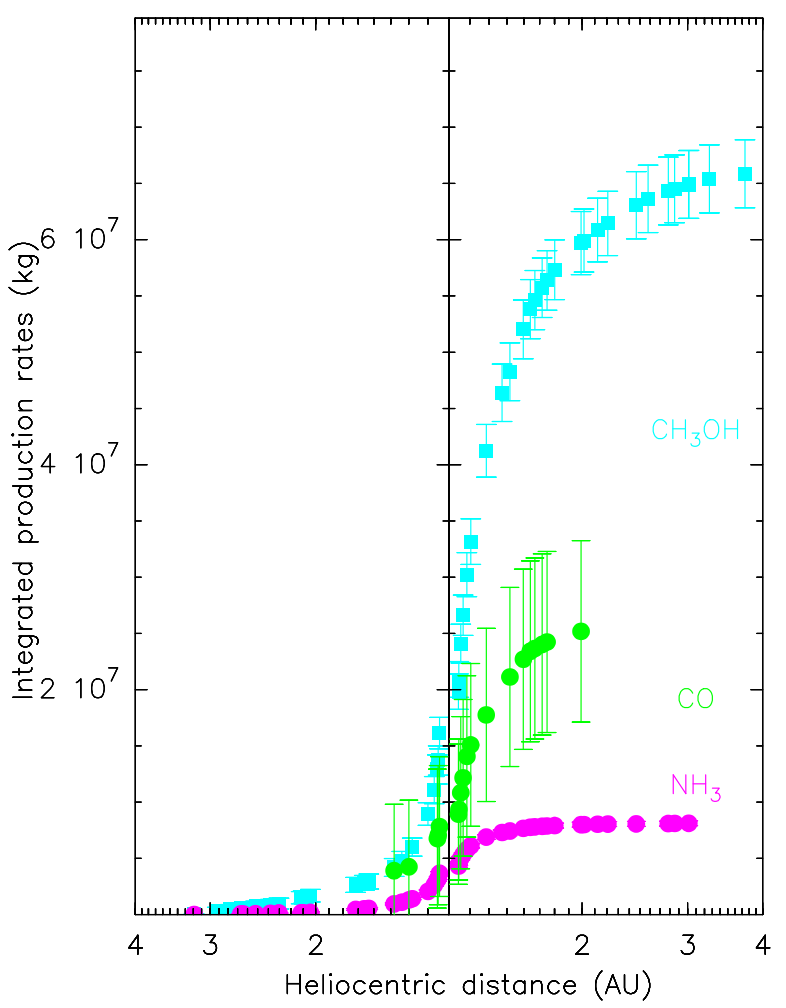

Fig. 25. Same as Fig. 24 for methanol, carbon monoxide, and ammonia. The vertical dotted lines indicate the times of the equinoxes (blue) and solstice (red). Only $25 \%$ of the mass of methanol and CO has been cumulatively lost at perihelion time. The emission of methanol does not decrease as quickly as for ammonia. The orbit-integrated mass losses at the last points are $66 \pm 3,25 \pm 8$, and $8.1 \pm 0.2 \times 10^{6} \mathrm{~kg}$ for $\mathrm{CH}_{3} \mathrm{OH}$, $\mathrm{CO}$, and $\mathrm{NH}_{3}$, respectively.

meter of surface erosion (Keller et al. 2017). As a consequence, the dust-to-gas ratio, equal to or an upper limit of the dust-toice ratio, in the material lost by the comet is $1.5 \pm 0.8$, including all errors. There is evidence for re-deposition, especially in the northern plains, of dusty material (larger particles; Keller et al. 2017), but the question is how much this represents relative to the mass loss. If the average dust-to-ice ratio of the nucleus significantly differs from what is ejected, it also means that it may no longer be representative to the original value because the nucleus is enriching in refractory or dusty material at each perihelion. For example, if the pristine dust-to-ice ratio is $8: 1$, and $2: 1$ in the lost material, this implies that $0.3 \%$ of the nucleus mass in pure dust falls back, budgetary speaking, increasing the global to dust-to-ice ratio (by $\sim 0.018$ ) at every perihelion passage. This means that it was about 7.5:1 only in 1963 when $67 \mathrm{P}$ reached its present orbit. This throws doubt on the original dust-to-ice ratio of the comet, especially if it has been active for several centuries as a Jupiter-family comet, after it left the Kuiper Belt.

\section{Conclusion}

We have presented the analysis of a large data set obtained with the MIRO instrument on board the Rosetta spacecraft. Our study focused on the eight molecular transitions in the submillimeter spectra. From mapping data of the inner atmosphere of comet $67 \mathrm{P}$, complemented by nadir data, we monitored the geometry of the outgassing and the production rates of $\mathrm{H}_{2} \mathrm{O}, \mathrm{CH}_{3} \mathrm{OH}, \mathrm{NH}_{3}$, and CO for a period covering June 2014 to June $2016\left(r_{\mathrm{h}}=1.24\right.$ 3.65 AU). The 3D spatial distribution of these molecules was deduced by fitting the Doppler velocity profiles and their variation across the coma. We used a simplified modeling that only included measurable parameters. The main results of this study are listed below.

- The latitudes of the source regions show that water and ammonia outgassing approximately followed the subsolar point, while methanol was released from the southernmost regions during an extended time period after perihelion. At the two solstices, the emission of all molecules originates farther poleward of the subsolar latitude.

- The spatial distribution of the molecules around the nucleus is anisotropic, and more than $90 \%$ of the molecules are concentrated in a Gaussian-like jet, with a FWHM ranging from $70^{\circ}-140^{\circ}$. The jet width evolves with season, is broadest around the pre-perihelion equinox and narrower before, when the main outgassing source was near the north pole, that is, the neck areas.

- The production rates displayed steep heliocentric variations $\left(\propto r_{\mathrm{h}}^{-4}\right.$ to $r_{\mathrm{h}}^{-8}$ ), which we explained by the strong activity of the southern hemisphere when it became illuminated. These strong seasonal effects are probably related to significant erosion in the southern hemisphere, along with accumulation of volatile-poor dust particles that fall back in the northern hemisphere.

- The mean abundances in the coma exhibited significant changes near perihelion and during the following months. Whereas the production rate ratio of ammonia to water slightly decreased as the comet receded from the Sun, the $\mathrm{CO} / \mathrm{H}_{2} \mathrm{O}$ and $\mathrm{CH}_{3} \mathrm{OH} / \mathrm{H}_{2} \mathrm{O}$ production rate ratios increased. In the range 1.24-3.2 AU post-perihelion, the abundance of methanol increased from 2 to $20 \%$.

- The $\mathrm{CO}$ and $\mathrm{CH}_{3} \mathrm{OH}$ sources are mainly located in southern areas and seem to correlate with the source regions of $\mathrm{CO}_{2}$. The sustained production of these molecules from the southernmost regions after perihelion can be explained by sublimation fronts closer to the surface. Indeed, southern regions were strongly eroded. We found that $95 \%$ of the mass lost by the nucleus was released between the equinoxes.

- There is no correlation between the behavior of $\mathrm{NH}_{3}$, $\mathrm{CH}_{3} \mathrm{OH}$, and $\mathrm{CO}$ and the sublimation temperature of the pure ices, in line with the conclusions of Gasc et al. (2017).

- The total loss rates over two years were measured individually for each molecules, giving $\mathrm{CH}_{3} \mathrm{OH} / \mathrm{H}_{2} \mathrm{O}=1.5 \pm 0.1 \%$, $\mathrm{NH}_{3} / \mathrm{H}_{2} \mathrm{O}=0.34 \pm 0.01 \%$, and $\mathrm{CO} / \mathrm{H}_{2} \mathrm{O}=0.6 \pm 0.2 \%$, in numbers.

- The total ice loss for the 2015 perihelion passage of comet $67 \mathrm{P}$, including molecules not observed with MIRO, is estimated to be $4.2 \pm 0.2 \times 10^{9} \mathrm{~kg}$, up to $5.4 \pm 0.4 \times 10^{9} \mathrm{~kg}$ in the maximum case when considering less confident nadir data analyses. From the total mass loss of the comet measured by the RSI experiment (Pätzold et al. 2019), we derive a dust/ice mass-loss of $1.5 \pm 0.8$.

- The abundances of $\mathrm{CH}_{3} \mathrm{OH}$ and $\mathrm{NH}_{3}$ measured around perihelion are in the mean of values measured for other comets. However, the $\mathrm{CO} / \mathrm{H}_{2} \mathrm{O}$ abundance is in the low end of measured values.

- The $\mathrm{H}_{2}^{18} \mathrm{O} / \mathrm{H}_{2}^{17} \mathrm{O}$ is not tightly constrained. The derived value of $5.5 \pm 0.8$ is consistent with VSMOW.

- There is no significant evidence for a distributed source of water, such as sublimating icy grains, within $20 \mathrm{~km}$ from nucleus center. By comparing MIRO measurements to remote observations performed with the Odin telescope, we conclude that the discrepancy of a factor of 2.5 between the water production rates of MIRO and ROSINA cannot 
Table 6. Integrated mass losses and relative abundances.

\begin{tabular}{lcccc}
\hline \hline & $\begin{array}{c}\mathrm{H}_{2} \mathrm{O} \\
\left(10^{9} \mathrm{~kg}\right)\end{array}$ & $\begin{array}{c}\mathrm{CH}_{3} \mathrm{OH} \\
\left(10^{6} \mathrm{~kg}\right)\end{array}$ & $\begin{array}{c}\mathrm{NH}_{3} \\
\left(10^{6} \mathrm{~kg}\right)\end{array}$ & $\begin{array}{c}\mathrm{CO} \\
\left(10^{6} \mathrm{~kg}\right)\end{array}$ \\
\hline \multicolumn{5}{c}{ Data based on analysis of mapping data } \\
\hline Interpolated & $2.42 \pm 0.04$ & $66 \pm 3$ & $8.1 \pm 0.2$ & $30 \pm 10^{(a)}$ \\
Power-law fits & $2.42 \pm 0.14$ & $64 \pm 12$ & $7.8 \pm 1.0$ & $24 \pm 12$ \\
& $100 \%$ & $1.53 \pm 0.08 \%$ & $0.35 \pm 0.01 \%$ & $0.75 \pm 0.24 \%$ \\
\hline \multicolumn{5}{c}{ Data based on analysis of nadir pointings } \\
\hline Interpolated & $3.30 \pm 0.19$ & $90 \pm 5$ & $10.5 \pm 0.7$ & $16 \pm 1.7^{(a)}$ \\
Power-law fits & $2.82 \pm 0.67$ & $76 \pm 21$ & $8.3 \pm 3.4$ & $13 \pm 7.6$ \\
& $100 \%$ & $1.53 \pm 0.13 \%$ & $0.34 \pm 0.03 \%$ & $0.31 \pm 0.04 \%$ \\
\hline
\end{tabular}

Notes. Relative abundances are given by numbers below the mass losses. ${ }^{(a)}$ Includes extrapolation to 3.8 AU pre- and post-perihelion following the behavior of $\mathrm{CH}_{3} \mathrm{OH}$ (i.e. adds $18.7 \pm 2.7 \%$ mass loss beyond $2 \mathrm{AU}$ from the Sun for mapping data and $+2.9 \%$ for nadir data).

be explained by this process. The contribution of a distributed source of water is at most $50 \%$ of the production rate measured by MIRO.

One central question in cometary science is the degree to which molecular abundances in cometary atmospheres are representative of the internal composition of the nucleus. The MIRO results on the activity of $67 \mathrm{P}$, combined with those obtained with other Rosetta instruments and thermophysical models, should help answering this question.

Acknowledgements. The authors acknowledge support from their institutions and funding sources. A part of the research was carried out at the Jet Propulsion Laboratory, California Institute of Technology, under a contract with the National Aeronautics and Space Administration. A part of the research was carried out at the Max-Planck-Institut für Sonnensystemforschung with financial support from Deutsches Zentrum für Luft- und Raumfarht and Max-Planck-Gesellschaft. Parts of the research were carried out by LESIA and LERMA, Observatoire de Paris, with financial support from CNES and CNRS/Institut des sciences de l'univers. A part of the research was carried out at the National Central University with funding from the Taiwanese National Science Counsel grant NSC 101-2111-M-008-016. A part of the research was carried out at the University of Massachusetts, Amherst, USA. We thank Y. Anderson, T. Koch, R. Nowicki, L. Pan, P. Springer and Lucas Kamp for their efforts in scheduling, operations, and support of the MIRO instrument. We specially want to thank all the MIRO engineers, researchers, CoI who participated to the development of the MIRO instrument and passed away since the beginning of the project/mission : we have our best thoughts for Sonia Khatri, Lucas Kamp and Mark Allen from JPL and Maurice Gheudin at Paris Observatory. We thank the Odin Team (A. Sandqvist, S. Lundin, U. Frisk, et al.) for scheduling special observations of comet 67P in support of the Rosetta mission in November 2015. Odin is a Swedish-led satellite project funded jointly by the Swedish National Space Board (SNSB), the Canadian Space Agency (CSA), the National Technology Agency of Finland (Tekes) and the Centre National d'Études Spatiales (CNES, France). The Swedish Space Corporation is the prime contractor, also responsible for Odin operations. The data were reduced and analyzed thanks to the use of the GILDAS, class software (http: //www . iram. fr/IRAMFR/GILDAS).

\section{References}

Accomazzo, A., Ferri, P., Lodiot, S., et al. 2016, Acta Astronaut., 126, 190 Accomazzo, A., Ferri, P., Lodiot, S., et al. 2017, Acta Astronaut., 136, 354 Altwegg, K., Balsiger, H., Bar-Nun, A., et al. 2015, Science, 347, 1261952 Bieler, A., Altwegg, K., Balsiger, H., et al., 2015, Nature, 526, 678

Biver, N., Bockelée-Morvan, D., Colom, P., et al. 2002, Earth Moon Planets, 90, 5

Biver, N., Bockelée-Morvan, D., Crovisier, J., et al. 2006, A\&A, 449, 1255

Biver, N., Bockelée-Morvan, D., Crovisier, J., et al. 2007, Planet. Space Sci., 55, 1058

Biver, N., Bockelée-Morvan, D., Colom, P., et al. 2011, A\&A, 528, A142

Biver, N., Crovisier, J., Bockelée-Morvan, D., et al. 2012, A\&A, 539, A68

Biver, N., Hofstadter, M., Gulkis, S., et al. 2015, A\&A, 583, A3

Biver, N., Moreno, R., Bockelée-Morvan, D., et al. 2016, A\&A, 589, A78
Bockelée-Morvan, D., \& Biver, N. 2017, Phil. Trans. R. Soc. A, 375, 20160252

Bockelée-Morvan, D., Crovisier, J., Mumma, M. J., \& Weaver, H. A. 2004, in Comets II, eds. M. C. Festou, H. U. Keller, \& H. A. Weaver (Tucson, AZ: University of Arizona Press), 391

Bockelée-Morvan, D., Biver, N., Swinyard, B., et al. 2012, A\&A, 544, L15

Bockelée-Morvan, D., Debout, V., Erard, S., et al. 2015a, A\&A, 583, A6

Bockelée-Morvan, D., Calmonte, U., Charnley, S., et al. 2015b, Space Sci. Rev., 197, 47

Bockelée-Morvan, D., Crovisier, J., Erard, S., et al. 2016, MNRAS, 462, S170

Calmonte, U., Altwegg, K., Balsiger, H., et al. 2016, MNRAS, 462, S253

Cazzoli, G., Dore, L., \& Puzzarini, C. 2009, A\&A, 507, 1707

Choukroun, M., Kheim, S., Schloerb, F. P., et al. 2015, A\&A, 583, A28

Crifo, J.-F., \& Slanina, Z. 1991, ApJ, 383, 351

Dello Russo, N., Kawakita, H., Vervack, R. J., \& Weaver, H. A. 2016, Icarus, 278, 301

Feldman, P. D., A’Hearn, M. F., Bertaux, J.-L., et al. 2018, AJ, 155, 9

Fougere, N., Altwegg, K., Berthelier, J.-J., et al. 2016, MNRAS, 462, S156

Frisk, U., Hagström, M., Ala-Laurinaho, J., et al. 2003, A\&A, 402, L27

Fulle, M., Blum, J., Green, S. F., et al. 2019, MNRAS, 482, 3326

Gasc, S., Altwegg, K., Balsiger, H., et al. 2017, MNRAS, 469, S108

Giorgini, J. D., Chamberlin, A. B., \& Park, S. B. JPL Solar System Dynamics Group, Horizons On-Line Ephemeris System (https://ssd. jpl .nasa. gov/horizons.cgi)

Grün, E., Agarwal, J., Altobelli, N., et al. 2016, MNRAS, 462, S220

Gulkis, S., Frerking, M., Crovisier, J., et al. 2007, Space Sci. Rev., 128, 56

Gulkis, S., Allen, M., von Allmen, P., et al. 2015, Science, 347, aaa0709

Hansen, K. C., Altwegg, K., Berthelier, J.-J., et al. 2016, MNRAS, 462, S491

Heritier, K. L., Henri, P., Vallières, X., et al. 2017, MNRAS, 469, S118

Hoang, M., Altwegg, K., Balsiger, H., et al. 2017, A\&A, 600, A77

Jorda, L., Gaskell, R., Capanna, C., et al. 2016, Icarus, 277, 257

Keller, H. U., Mottola, S., Hviid, S. F., et al. 2017, MNRAS, 469, S357

Koschny, D., Dhiri, V., Wirth, K., et al. 2007, Space Sci. Rev., 128, 167

Läuter, M., Kramer, T., Rubin, M., \& Altwegg, K. 2019, MNRAS, 483, 852

Le Roy, L., Altwegg, K., Balsiger, H., et al. 2015, A\&A, 583, A1

Lee, S., von Allmen, P., Allen, M., et al. 2015, A\&A, 583, A5

Marshall, D. W., Hartogh, P., Rezac, L., et al. 2017, A\&A, 603, A87

Migliorini, A., Piccioni, G., Capaccioni, F., et al. 2016, A\&A, 589, A45

Mumma, M. J., \& Charnley, S. B. 2011, ARA\&A, 49, 471

Nordh, H. L., von Schéele, F., Frisk, U., et al. 2003, A\&A, 402, L21

Paquette, J. A., Engrand, C., Hilchenbach, M., et al. 2018, MNRAS, 477, 3836

Pätzold, M., Andert, T. P., Hahn, M., et al. 2019, MNRAS, 483, 2337

Peusker, F., Scholten, F., Matz, K.-D., et al. 2017, A\&A, 607, L1

Puzzarini, C., Cazzoli, G., Harding, M. E., Vázquez, J., \& Gauss, J. 2009, J. Chem. Phys., 131, 234304

Schloerb, P., Spilker, T., Lellouch, E., et al. 2015, A\&A, 583, A29

Schroeder, I. R. H. G., Altwegg, K., Balsiger, H., et al. 2019, A\&A, 630, A29 (Rosetta 2 SI)

Snodgrass, C., A'Hearn, M. F., Aceituno, F., et al. 2016, Phil. Trans. R. Soc. London, Ser. A, 375, 2097

Vincent, J.-B., A'Hearn, M. F., Lin, Z.-Y., et al. 2016, MNRAS, 462, 184

Yamamoto, T. 1985, A\&A, 142, 31

Zakharov, V., Bockelée-Morvan, D., Biver, N., Crovisier, J., \& Lecacheux, A 2007, A\&A, 473, 303 


\section{Appendix A: Additional tables}

Table A.1. Inner coma maps of comet 67P/Churyumov-Gerasimenko.

\begin{tabular}{|c|c|c|c|c|c|c|c|c|c|}
\hline \multirow{2}{*}{$\begin{array}{c}\text { Date } \\
\text { yyyy/mm/dd.dd-dd.dd }\end{array}$} & \multirow{2}{*}{$\begin{array}{c}\text { UT range } \\
\text { hh.hh-hh.hh }\end{array}$} & \multirow{2}{*}{$\begin{array}{c}r_{\mathrm{h}} \\
(\mathrm{AU}) \\
\end{array}$} & \multirow{2}{*}{$\begin{array}{l}\Delta^{(a)} \\
(\mathrm{km})\end{array}$} & \multicolumn{2}{|c|}{ Sun } & \multicolumn{2}{|c|}{ North pole } & \multicolumn{2}{|c|}{ Map coverage } \\
\hline & & & & $\mathrm{PA}$ & Phase & PA & Phase & in $X(\mathrm{~km})$ & in $Y(\mathrm{~km})$ \\
\hline $2014 / 07 / 29.19-29.34$ & $4.58-8.12$ & 3.645 & 1818.5 & $90^{\circ}$ & $1.5^{\circ}$ & $4.2^{\circ}$ & $45.1^{\circ}$ & 30 & 31 \\
\hline $2014 / 09 / 07.44-07.60$ & $10.50-14.40$ & 3.410 & 58.3 & $90^{\circ}$ & $90.4^{\circ}$ & $42.8^{\circ}$ & $95.6^{\circ}$ & 5 & 5 \\
\hline $2014 / 10 / 12.77-13.41$ & $18.62-9.55$ & 3.190 & 18.4 & $90^{\circ}$ & $91.7^{\circ}$ & $40.7^{\circ}$ & $99.0^{\circ}$ & 5 & $5^{(c)}$ \\
\hline $2014 / 11 / 23.03-23.33$ & $0.80-7.98$ & 2.920 & 30.8 & $90^{\circ}$ & $93.8^{\circ}$ & $112.4^{\circ}$ & $144.5^{\circ}$ & 12 & $11^{(b)}$ \\
\hline 2014/12/13.55-13.95 & $13.15-22.76$ & 2.777 & 19.3 & $90^{\circ}$ & $89.9^{\circ}$ & $86.2^{\circ}$ & $145.5^{\circ}$ & 4.5 & $8.5^{(b)}$ \\
\hline 2015/01/01.03-02.01 & $0.68-24.21$ & 2.643 & 28.5 & $90^{\circ}$ & $96.2^{\circ}$ & $32.0^{\circ}$ & $115.4^{\circ}$ & 16.8 & $14.6^{(b)}$ \\
\hline $2015 / 01 / 18.05-18.65$ & $1.23-15.64$ & 2.520 & 28.5 & $270^{\circ}$ & $93.8^{\circ}$ & $321.2^{\circ}$ & $135.3^{\circ}$ & 18 & $16^{(b)}$ \\
\hline $2015 / 02 / 15.87-16.07$ & $20.94-1.76$ & 2.306 & 151.5 & $90^{\circ}$ & $90.0^{\circ}$ & $23.7^{\circ}$ & $91.6^{\circ}$ & 12.2 & 11.2 \\
\hline $2015 / 02 / 16.13-16.50$ & $3.17-11.90$ & 2.303 & 184.0 & $90^{\circ}$ & $90.4^{\circ}$ & $23.6^{\circ}$ & $91.8^{\circ}$ & 15.0 & 14.6 \\
\hline $2015 / 03 / 14.63-14.80$ & $15.14-19.25$ & 2.101 & 79.2 & $270^{\circ}$ & $50.0^{\circ}$ & $19.7^{\circ}$ & $42.1^{\circ}$ & 17.6 & 11.0 \\
\hline 2015/03/14.86-15.01 & $20.72-24.29$ & 2.100 & 76.3 & $270^{\circ}$ & $47.9^{\circ}$ & $27.2^{\circ}$ & $39.4^{\circ}$ & 21.3 & 10.6 \\
\hline $2015 / 03 / 22.05-22.44$ & $1.12-10.46$ & 2.043 & 79.1 & $90^{\circ}$ & $54.9^{\circ}$ & $60.1^{\circ}$ & $122.2^{\circ}$ & 10.6 & 9.8 \\
\hline $2015 / 03 / 22.54-22.79$ & $12.90-18.92$ & 2.040 & 77.8 & $90^{\circ}$ & $56.2^{\circ}$ & $71.6^{\circ}$ & $127.8^{\circ}$ & 8.0 & 7.0 \\
\hline $2015 / 05 / 15.55-15.95$ & $13.89-22.89$ & 1.632 & 129.5 & $270^{\circ}$ & $65.5^{\circ}$ & $75.7^{\circ}$ & $27.5^{\circ}$ & 11.3 & $11.3^{(b)}$ \\
\hline 2015/06/06.89-07.38 & $21.28-9.19$ & 1.481 & 202.0 & $270^{\circ}$ & $86.8^{\circ}$ & $12.0^{\circ}$ & $109.6^{\circ}$ & $3.9^{(c)}$ & $3.9^{(c)}$ \\
\hline $2015 / 06 / 14.05-14.12$ & $1.22-2.81$ & 1.439 & 201.4 & $270^{\circ}$ & $87.9^{\circ}$ & $23.3^{\circ}$ & $49.1^{\circ}$ & 10.8 & 9.9 \\
\hline $2015 / 06 / 14.16-14.22$ & $3.74-5.31$ & 1.439 & 201.8 & $270^{\circ}$ & $87.9^{\circ}$ & $23.8^{\circ}$ & $48.1^{\circ}$ & 10.8 & 9.9 \\
\hline 2015/06/14.29-14.34 & $6.92-8.20$ & 1.438 & 202.3 & $270^{\circ}$ & $88.0^{\circ}$ & $24.4^{\circ}$ & $46.9^{\circ}$ & 11.6 & 10.6 \\
\hline 2015/06/14.38-14.44 & $9.10-10.49$ & 1.438 & 202.8 & $270^{\circ}$ & $88.0^{\circ}$ & $24.9^{\circ}$ & $46.1^{\circ}$ & 11.7 & 10.6 \\
\hline $2015 / 06 / 24.45-24.60$ & $10.88-14.48$ & 1.382 & 187.0 & $270^{\circ}$ & $89.8^{\circ}$ & $34.1^{\circ}$ & $41.4^{\circ}$ & 14.2 & 13.2 \\
\hline $2015 / 06 / 24.88-25.03$ & $21.15-24.68$ & 1.380 & 173.6 & $270^{\circ}$ & $89.8^{\circ}$ & $29.3^{\circ}$ & $50.1^{\circ}$ & 13.2 & 12.2 \\
\hline $2015 / 07 / 13.12-13.27$ & $2.95-6.50$ & 1.300 & 153.8 & $270^{\circ}$ & $88.8^{\circ}$ & $34.3^{\circ}$ & $73.2^{\circ}$ & 14.3 & 12.7 \\
\hline $2015 / 07 / 23.31-23.46$ & $7.36-10.96$ & 1.269 & 173.8 & $270^{\circ}$ & $88.9^{\circ}$ & $40.2^{\circ}$ & $74.4^{\circ}$ & 22.5 & 20.3 \\
\hline $2015 / 07 / 30.31-30.46$ & $7.33-10.95$ & 1.254 & 179.1 & $90^{\circ}$ & $90.0^{\circ}$ & $264.5^{\circ}$ & $138.0^{\circ}$ & 13.5 & 12.4 \\
\hline $2015 / 07 / 30.49-30.65$ & $11.78-15.69$ & 1.254 & 180.2 & $90^{\circ}$ & $90.0^{\circ}$ & $269.6^{\circ}$ & $138.1^{\circ}$ & 27.2 & 25.1 \\
\hline $2015 / 07 / 30.74-30.88$ & $17.67-21.22$ & 1.254 & 183.0 & $90^{\circ}$ & $90.0^{\circ}$ & $275.7^{\circ}$ & $137.8^{\circ}$ & 18.4 & 16.1 \\
\hline $2015 / 08 / 02.05-02.21$ & $1.24-5.03$ & 1.250 & 207.7 & $90^{\circ}$ & $89.4^{\circ}$ & $308.0^{\circ}$ & $120.7^{\circ}$ & 15.7 & 14.4 \\
\hline 2015/08/02.29-02.43 & $6.93-10.37$ & 1.250 & 206.9 & $90^{\circ}$ & $89.3^{\circ}$ & $309.6^{\circ}$ & $118.3^{\circ}$ & 21.0 & 18.3 \\
\hline 2015/08/08.87-09.01 & $20.83-24.22$ & 1.244 & 304.3 & $90^{\circ}$ & $89.3^{\circ}$ & $307.1^{\circ}$ & $65.8^{\circ}$ & 28.3 & 26.4 \\
\hline 2015/08/09.05-09.21 & $1.24-5.09$ & 1.244 & 305.3 & $90^{\circ}$ & $89.2^{\circ}$ & $306.4^{\circ}$ & $64.9^{\circ}$ & 34.0 & 32.0 \\
\hline $2015 / 08 / 17.54-17.69$ & $13.03-16.55$ & 1.245 & 328.2 & $270^{\circ}$ & $89.2^{\circ}$ & $66.2^{\circ}$ & $56.9^{\circ}$ & 30.4 & 28.6 \\
\hline 2015/08/17.72-17.86 & $17.34-20.71$ & 1.245 & 328.7 & $270^{\circ}$ & $89.3^{\circ}$ & $65.3^{\circ}$ & $57.5^{\circ}$ & 30.6 & 28.9 \\
\hline $2015 / 08 / 20.07-20.24$ & $1.64-5.76$ & 1.246 & 326.3 & $270^{\circ}$ & $89.6^{\circ}$ & $55.4^{\circ}$ & $69.1^{\circ}$ & 35.6 & 32.2 \\
\hline $2015 / 08 / 20.31-20.43$ & $7.42-10.38$ & 1.247 & 325.5 & $270^{\circ}$ & $89.6^{\circ}$ & $54.8^{\circ}$ & $70.6^{\circ}$ & 32.6 & 25.0 \\
\hline $2015 / 08 / 27.31-27.46$ & $7.42-10.96$ & 1.256 & 400.8 & $270^{\circ}$ & $78.4^{\circ}$ & $63.2^{\circ}$ & $132.0^{\circ}$ & 41.2 & 36.6 \\
\hline 2015/08/27.49-27.67 & $11.86-16.02$ & 1.256 & 400.5 & $270^{\circ}$ & $77.5^{\circ}$ & $64.5^{\circ}$ & $134.0^{\circ}$ & 44.4 & 39.8 \\
\hline 2015/08/27.73-27.88 & $17.62-21.18$ & 1.257 & 401.2 & $270^{\circ}$ & $76.4^{\circ}$ & $66.1^{\circ}$ & $136.3^{\circ}$ & 40.8 & 37.0 \\
\hline $2015 / 09 / 01.56-01.73$ & $13.49-17.60$ & 1.267 & 422.0 & $90^{\circ}$ & $70.6^{\circ}$ & $299.0^{\circ}$ & $139.3^{\circ}$ & 38.0 & 36.5 \\
\hline 2015/09/01.80-01.94 & $19.19-22.57$ & 1.267 & 427.0 & $90^{\circ}$ & $70.8^{\circ}$ & $300.3^{\circ}$ & $138.0^{\circ}$ & 39.0 & 37.0 \\
\hline $2015 / 09 / 08.58-08.74$ & $13.85-17.64$ & 1.286 & 328.6 & $90^{\circ}$ & $117.3^{\circ}$ & $298.8^{\circ}$ & $30.2^{\circ}$ & 35.0 & 30.0 \\
\hline 2015/09/08.80-08.89 & $19.25-21.43$ & 1.286 & 333.6 & $90^{\circ}$ & $118.0^{\circ}$ & $295.5^{\circ}$ & $28.0^{\circ}$ & 26.6 & 22.3 \\
\hline $2015 / 09 / 14.12-14.27$ & $2.98-6.38$ & 1.304 & 312.3 & $270^{\circ}$ & $103.2^{\circ}$ & $53.3^{\circ}$ & $57.9^{\circ}$ & 23.6 & 21.2 \\
\hline $2015 / 09 / 14.31-14.44$ & $7.34-10.50$ & 1.305 & 313.0 & $270^{\circ}$ & $101.8^{\circ}$ & $53.3^{\circ}$ & $59.9^{\circ}$ & 22.8 & 20.1 \\
\hline 2015/09/14.54-14.69 & $12.98-16.47$ & 1.306 & 314.5 & $270^{\circ}$ & $100.0^{\circ}$ & $53.3^{\circ}$ & $62.7^{\circ}$ & 17.4 & 16.3 \\
\hline
\end{tabular}

Notes. ${ }^{(a)}$ Distance Rosetta-comet. ${ }^{(b)}$ Cross-scan across the coma along several radii (at least four every $90^{\circ}$ in PA). ${ }^{(c)}$ Circular coma scan around the nucleus (at 3.5-4.5 km projected distance). 
Table A.1. continued.

\begin{tabular}{|c|c|c|c|c|c|c|c|c|c|}
\hline \multirow{2}{*}{$\begin{array}{c}\text { Date } \\
\text { yyyy/mm/dd.dd-dd.dd }\end{array}$} & \multirow{2}{*}{$\begin{array}{c}\text { UT range } \\
\text { hh.hh-hh.hh }\end{array}$} & \multirow{2}{*}{$\begin{array}{c}r_{\mathrm{h}} \\
(\mathrm{AU}) \\
\end{array}$} & \multirow{2}{*}{$\begin{array}{l}\Delta^{(a)} \\
(\mathrm{km})\end{array}$} & \multicolumn{2}{|c|}{ Sun } & \multicolumn{2}{|c|}{ North pole } & \multicolumn{2}{|c|}{ Map coverage } \\
\hline & & & & PA & Phase & PA & Phase & in $X(\mathrm{~km})$ & in $Y(\mathrm{~km})$ \\
\hline 2015/10/01.92-02.09 & $21.97-2.29$ & 1.383 & 1254 & $270^{\circ}$ & $51.1^{\circ}$ & $29.1^{\circ}$ & $147.3^{\circ}$ & 44.6 & 43.8 \\
\hline $2015 / 10 / 02.16-02.31$ & $3.74-7.50$ & 1.384 & 1224 & $270^{\circ}$ & $51.3^{\circ}$ & $29.2^{\circ}$ & $146.8^{\circ}$ & 43.5 & 42.8 \\
\hline $2015 / 10 / 02.35-02.52$ & $8.28-12.58$ & 1.386 & 1197 & $270^{\circ}$ & $51.5^{\circ}$ & $29.3^{\circ}$ & $146.3^{\circ}$ & 38.3 & 37.6 \\
\hline $2015 / 10 / 02.59-02.74$ & $14.06-17.70$ & 1.387 & 1167 & $270^{\circ}$ & $51.6^{\circ}$ & $29.4^{\circ}$ & $145.6^{\circ}$ & 33.3 & 32.5 \\
\hline $2015 / 10 / 07.29-07.39$ & $7.01-9.37$ & 1.412 & 648 & $270^{\circ}$ & $59.9^{\circ}$ & $37.4^{\circ}$ & $121.9^{\circ}$ & 27.9 & 26.0 \\
\hline $2015 / 10 / 07.46-07.60$ & $10.98-14.47$ & 1.413 & 641 & $270^{\circ}$ & $59.9^{\circ}$ & $37.4^{\circ}$ & $121.3^{\circ}$ & 35.5 & 32.5 \\
\hline $2015 / 10 / 07.88-07.93$ & $21.20-22.35$ & 1.415 & 628 & $270^{\circ}$ & $59.8^{\circ}$ & $37.6^{\circ}$ & $120.2^{\circ}$ & 18.2 & 16.5 \\
\hline 2015/10/07.98-08.03 & $23.60-24.73$ & 1.416 & 625 & $270^{\circ}$ & $59.8^{\circ}$ & $37.5^{\circ}$ & $120.0^{\circ}$ & 18.2 & 16.4 \\
\hline $2015 / 10 / 17.28-17.39$ & $6.60-9.25$ & 1.471 & 443.1 & $270^{\circ}$ & $66.2^{\circ}$ & $61.5^{\circ}$ & $74.3^{\circ}$ & 20.6 & 19.3 \\
\hline $2015 / 10 / 17.45-17.59$ & $10.82-14.07$ & 1.472 & 441.6 & $270^{\circ}$ & $66.0^{\circ}$ & $62.0^{\circ}$ & $74.1^{\circ}$ & 22.6 & 21.7 \\
\hline 2015/10/17.63-17.79 & $15.18-19.04$ & 1.473 & 440.1 & $270^{\circ}$ & $65.9^{\circ}$ & $62.5^{\circ}$ & $73.8^{\circ}$ & 25.0 & 23.8 \\
\hline $2015 / 10 / 24.28-24.39$ & $6.60-9.38$ & 1.515 & 385.6 & $90^{\circ}$ & $63.3^{\circ}$ & $302.9^{\circ}$ & $77.1^{\circ}$ & 18.1 & 16.8 \\
\hline $2015 / 10 / 24.45-24.58$ & $10.82-14.02$ & 1.516 & 376.7 & $90^{\circ}$ & $62.8^{\circ}$ & $304.2^{\circ}$ & $78.4^{\circ}$ & 19.2 & 18.4 \\
\hline $2015 / 10 / 24.63-24.80$ & $15.13-19.28$ & 1.518 & 367.4 & $90^{\circ}$ & $62.3^{\circ}$ & $305.6^{\circ}$ & $79.9^{\circ}$ & 21.5 & 20.4 \\
\hline $2015 / 10 / 24.87-25.00$ & $20.82-24.07$ & 1.519 & 357.9 & $90^{\circ}$ & $61.8^{\circ}$ & $307.1^{\circ}$ & $81.7^{\circ}$ & 18.4 & 17.4 \\
\hline $2015 / 11 / 05.07-05.22$ & $1.58-5.38$ & 1.595 & 237.1 & $270^{\circ}$ & $60.8^{\circ}$ & $22.5^{\circ}$ & $129.7^{\circ}$ & 13.9 & 13.3 \\
\hline $2015 / 11 / 05.31-05.45$ & $7.41-10.83$ & 1.596 & 233.3 & $270^{\circ}$ & $60.6^{\circ}$ & $23.4^{\circ}$ & $125.9^{\circ}$ & 12.4 & 11.7 \\
\hline 2015/11/05.49-05.66 & $11.80-15.93$ & 1.598 & 231.2 & $270^{\circ}$ & $60.5^{\circ}$ & $24.3^{\circ}$ & $122.5^{\circ}$ & 16.8 & 16.1 \\
\hline 2015/11/05.73-05.88 & $17.57-21.10$ & 1.600 & 230.1 & $270^{\circ}$ & $60.5^{\circ}$ & $25.6^{\circ}$ & $118.4^{\circ}$ & 16.5 & 16.1 \\
\hline $2015 / 11 / 11.29-11.40$ & $7.02-9.55$ & 1.639 & 199.8 & $270^{\circ}$ & $62.9^{\circ}$ & $74.5^{\circ}$ & $61.5^{\circ}$ & 11.1 & 10.5 \\
\hline $2015 / 11 / 11.45-11.61$ & $10.90-14.65$ & 1.640 & 194.2 & $270^{\circ}$ & $62.3^{\circ}$ & $77.4^{\circ}$ & $61.3^{\circ}$ & 14.1 & 13.5 \\
\hline 2015/11/11.64-11.82 & $15.33-19.57$ & 1.641 & 189.4 & $270^{\circ}$ & $61.7^{\circ}$ & $80.7^{\circ}$ & $61.2^{\circ}$ & 11.0 & 10.6 \\
\hline $2015 / 11 / 15.29-15.44$ & $6.93-10.45$ & 1.668 & 155.1 & $90^{\circ}$ & $60.7^{\circ}$ & $316.0^{\circ}$ & $80.3^{\circ}$ & 11.2 & 10.7 \\
\hline $2015 / 11 / 15.49-15.64$ & $11.79-15.30$ & 1.669 & 152.2 & $90^{\circ}$ & $60.4^{\circ}$ & $317.8^{\circ}$ & $82.3^{\circ}$ & 11.0 & 10.5 \\
\hline 2015/11/15.71-15.85 & $16.98-20.50$ & 1.671 & 149.4 & $90^{\circ}$ & $60.1^{\circ}$ & $319.6^{\circ}$ & $84.6^{\circ}$ & 10.8 & 10.4 \\
\hline $2015 / 11 / 21.87-22.02$ & $20.80-24.38$ & 1.716 & 136.3 & $270^{\circ}$ & $89.4^{\circ}$ & $106.2^{\circ}$ & $150.6^{\circ}$ & 10.0 & 9.5 \\
\hline $2015 / 11 / 22.05-22.22$ & $1.24-5.30$ & 1.717 & 133.8 & $270^{\circ}$ & $89.3^{\circ}$ & $101.5^{\circ}$ & $151.4^{\circ}$ & 7.9 & 7.5 \\
\hline $2015 / 11 / 22.29-22.43$ & $6.93-10.38$ & 1.719 & 131.5 & $270^{\circ}$ & $89.3^{\circ}$ & $95.3^{\circ}$ & $152.1^{\circ}$ & 9.5 & 9.1 \\
\hline $2015 / 11 / 26.07-26.24$ & $1.64-5.85$ & 1.747 & 123.6 & $270^{\circ}$ & $90.1^{\circ}$ & $30.8^{\circ}$ & $116.6^{\circ}$ & 11.1 & 7.1 \\
\hline $2015 / 11 / 26.31-26.46$ & $7.41-11.08$ & 1.749 & 122.2 & $270^{\circ}$ & $90.1^{\circ}$ & $29.6^{\circ}$ & $112.3^{\circ}$ & 13.8 & 12.9 \\
\hline $2015 / 11 / 26.49-26.67$ & $11.85-16.05$ & 1.750 & 121.7 & $270^{\circ}$ & $90.1^{\circ}$ & $28.9^{\circ}$ & $108.7^{\circ}$ & 11.0 & 7.1 \\
\hline $2015 / 11 / 26.73-26.88$ & $17.56-21.15$ & 1.752 & 122.1 & $270^{\circ}$ & $90.0^{\circ}$ & $28.0^{\circ}$ & $104.1^{\circ}$ & 13.7 & 12.9 \\
\hline $2015 / 12 / 03.07-03.24$ & $1.57-5.82$ & 1.799 & 103.1 & $90^{\circ}$ & $89.9^{\circ}$ & $267.1^{\circ}$ & $25.1^{\circ}$ & 15.2 & 9.6 \\
\hline $2015 / 12 / 03.31-03.46$ & $7.34-10.94$ & 1.801 & 101.3 & $90^{\circ}$ & $89.9^{\circ}$ & $277.5^{\circ}$ & $25.3^{\circ}$ & 11.3 & 10.6 \\
\hline $2015 / 12 / 03.49-03.67$ & $11.74-16.09$ & 1.802 & 100.2 & $90^{\circ}$ & $89.8^{\circ}$ & $285.7^{\circ}$ & $26.1^{\circ}$ & 11.3 & 10.0 \\
\hline $2015 / 12 / 03.73-03.88$ & $17.63-21.17$ & 1.804 & 99.7 & $90^{\circ}$ & $89.8^{\circ}$ & $295.6^{\circ}$ & $27.9^{\circ}$ & 11.2 & 10.6 \\
\hline $2015 / 12 / 22.61-22.74$ & $14.69-17.67$ & 1.948 & 89.5 & $90^{\circ}$ & $89.9^{\circ}$ & $218.0^{\circ}$ & $32.3^{\circ}$ & 17.5 & 15.0 \\
\hline $2015 / 12 / 22.80-22.94$ & $19.21-22.60$ & 1.950 & 91.0 & $90^{\circ}$ & $89.9^{\circ}$ & $220.9^{\circ}$ & $30.0^{\circ}$ & 13.0 & 8.2 \\
\hline $2015 / 12 / 23.30-23.40$ & 7.10-9.48 & 1.953 & 88.3 & $90^{\circ}$ & $89.9^{\circ}$ & $230.6^{\circ}$ & $24.9^{\circ}$ & 16.0 & 13.5 \\
\hline 2015/12/23.64-23.81 & $15.42-19.54$ & 1.956 & 83.1 & $90^{\circ}$ & $89.8^{\circ}$ & $243.7^{\circ}$ & $21.3^{\circ}$ & 12.5 & 9.0 \\
\hline $2015 / 12 / 28.12-28.27$ & $2.93-6.59$ & 1.991 & 76.6 & $90^{\circ}$ & $88.9^{\circ}$ & $341.5^{\circ}$ & $74.9^{\circ}$ & 8.2 & 6.0 \\
\hline $2015 / 12 / 28.34-28.48$ & $8.05-11.63$ & 1.993 & 77.5 & $90^{\circ}$ & $88.9^{\circ}$ & $341.9^{\circ}$ & $78.0^{\circ}$ & 8.6 & 7.5 \\
\hline $2015 / 12 / 28.54-28.69$ & $13.04-16.65$ & 1.994 & 78.6 & $90^{\circ}$ & $89.0^{\circ}$ & $342.2^{\circ}$ & $81.0^{\circ}$ & 8.5 & 6.2 \\
\hline $2015 / 12 / 31.07-31.24$ & $1.64-5.76$ & 2.014 & 80.4 & $90^{\circ}$ & $89.2^{\circ}$ & $341.3^{\circ}$ & $119.3^{\circ}$ & 15.7 & 13.8 \\
\hline $2015 / 12 / 31.31-31.46$ & $7.34-10.92$ & 2.016 & 79.5 & $90^{\circ}$ & $89.1^{\circ}$ & $342.8^{\circ}$ & $124.0^{\circ}$ & 12.0 & 6.9 \\
\hline 2015/12/31.49-31.67 & $11.80-16.10$ & 2.018 & 79.2 & $90^{\circ}$ & $89.1^{\circ}$ & $339.4^{\circ}$ & $128.1^{\circ}$ & 11.5 & 7.3 \\
\hline $2016 / 01 / 11.55-11.69$ & $13.09-16.57$ & 2.102 & 81.5 & $270^{\circ}$ & $89.6^{\circ}$ & $18.1^{\circ}$ & $51.5^{\circ}$ & 12.3 & 7.2 \\
\hline 2016/01/12.80-12.94 & $19.25-22.68$ & 2.112 & 88.1 & $270^{\circ}$ & $89.9^{\circ}$ & $21.9^{\circ}$ & $39.1^{\circ}$ & 18.0 & 15.5 \\
\hline $2016 / 01 / 14.07-14.24$ & $1.65-5.74$ & 2.122 & 84.9 & $90^{\circ}$ & $80.5^{\circ}$ & $224.1^{\circ}$ & $32.4^{\circ}$ & 16.4 & 14.7 \\
\hline 2016/01/14.31-14.46 & $7.32-10.95$ & 2.123 & 84.6 & $90^{\circ}$ & $78.4^{\circ}$ & $229.8^{\circ}$ & $32.1^{\circ}$ & 16.3 & 14.7 \\
\hline $2016 / 01 / 14.49-14.66$ & $11.85-15.89$ & 2.125 & 84.6 & $90^{\circ}$ & $76.7^{\circ}$ & $234.7^{\circ}$ & $32.0^{\circ}$ & 12.7 & 8.0 \\
\hline 2016/01/14.73-14.88 & $17.56-21.14$ & 2.127 & 84.8 & $90^{\circ}$ & $74.8^{\circ}$ & $240.6^{\circ}$ & $32.2^{\circ}$ & 11.3 & 6.2 \\
\hline
\end{tabular}


Table A.1. continued.

\begin{tabular}{|c|c|c|c|c|c|c|c|c|c|}
\hline \multirow{2}{*}{$\begin{array}{c}\text { Date } \\
\text { yyyy/mm/dd.dd-dd.dd }\end{array}$} & \multirow{2}{*}{$\begin{array}{c}\text { UT range } \\
\text { hh.hh-hh.hh }\end{array}$} & \multirow{2}{*}{$\begin{array}{c}r_{\mathrm{h}} \\
(\mathrm{AU}) \\
\end{array}$} & \multirow{2}{*}{$\begin{array}{l}\Delta^{(a)} \\
(\mathrm{km})\end{array}$} & \multicolumn{2}{|c|}{ Sun } & \multicolumn{2}{|c|}{ North pole } & \multicolumn{2}{|c|}{ Map coverage } \\
\hline & & & & PA & Phase & PA & Phase & in $X(\mathrm{~km})$ & in $\mathrm{Y}(\mathrm{km})$ \\
\hline $2016 / 01 / 14.92-15.09$ & $21.97-2.23$ & 2.128 & 85.2 & $90^{\circ}$ & $72.9^{\circ}$ & $246.0^{\circ}$ & $32.6^{\circ}$ & 10.5 & 9.4 \\
\hline 2016/01/15.16-15.31 & $3.85-7.47$ & 2.130 & 85.8 & $90^{\circ}$ & $71.2^{\circ}$ & $251.4^{\circ}$ & $33.3^{\circ}$ & 9.8 & 8.8 \\
\hline $2016 / 01 / 15.35-15.52$ & $8.29-12.49$ & 2.132 & 86.5 & $90^{\circ}$ & $69.7^{\circ}$ & $255.9^{\circ}$ & $34.1^{\circ}$ & 10.6 & 9.7 \\
\hline $2016 / 01 / 25.13-25.27$ & $3.03-6.52$ & 2.207 & 71.2 & $270^{\circ}$ & $59.8^{\circ}$ & $343.7^{\circ}$ & $134.9^{\circ}$ & 13.8 & 12.3 \\
\hline $2016 / 01 / 25.30-25.47$ & $7.30-11.38$ & 2.208 & 71.0 & $270^{\circ}$ & $59.8^{\circ}$ & $341.3^{\circ}$ & $137.5^{\circ}$ & 10.7 & 6.8 \\
\hline $2016 / 01 / 25.54-25.69$ & $13.03-16.64$ & 2.210 & 71.0 & $270^{\circ}$ & $60.0^{\circ}$ & $338.2^{\circ}$ & $140.6^{\circ}$ & 9.5 & 5.3 \\
\hline $2016 / 02 / 06.27-06.39$ & $6.50-9.33$ & 2.299 & 51.6 & $90^{\circ}$ & $64.3^{\circ}$ & $21.3^{\circ}$ & $141.8^{\circ}$ & 14.2 & 3.6 \\
\hline $2016 / 02 / 06.45-06.60$ & $10.78-14.35$ & 2.301 & 50.7 & $90^{\circ}$ & $63.2^{\circ}$ & $25.8^{\circ}$ & $144.0^{\circ}$ & 14.2 & 4.8 \\
\hline $2016 / 02 / 06.63-06.80$ & $15.18-19.19$ & 2.302 & 49.8 & $90^{\circ}$ & $62.3^{\circ}$ & $30.8^{\circ}$ & $146.4^{\circ}$ & 7.5 & 4.9 \\
\hline 2016/02/06.87-07.01 & $20.82-24.22$ & 2.304 & 48.8 & $90^{\circ}$ & $61.2^{\circ}$ & $37.5^{\circ}$ & $148.9^{\circ}$ & 7.3 & 5.2 \\
\hline $2016 / 02 / 15.54-15.69$ & $13.03-16.62$ & 2.369 & 38.7 & $270^{\circ}$ & $60.6^{\circ}$ & $337.2^{\circ}$ & $134.7^{\circ}$ & 10.8 & 3.3 \\
\hline $2016 / 02 / 15.72-15.90$ & $17.40-21.58$ & 2.371 & 38.6 & $270^{\circ}$ & $61.0^{\circ}$ & $333.1^{\circ}$ & $138.9^{\circ}$ & 5.9 & 3.5 \\
\hline $2016 / 02 / 15.96-16.11$ & $23.05-2.66$ & 2.373 & 38.6 & $270^{\circ}$ & $61.8^{\circ}$ & $328.2^{\circ}$ & $143.5^{\circ}$ & 5.8 & 2.9 \\
\hline $2016 / 02 / 16.38-16.53$ & $9.19-12.75$ & 2.376 & 38.9 & $270^{\circ}$ & $63.8^{\circ}$ & $317.3^{\circ}$ & $152.0^{\circ}$ & 5.8 & 3.0 \\
\hline $2016 / 02 / 27.87-28.01$ & $20.87-24.31$ & 2.462 & 29.4 & $90^{\circ}$ & $94.1^{\circ}$ & $357.2^{\circ}$ & $76.1^{\circ}$ & 6.7 & 5.9 \\
\hline $2016 / 02 / 28.05-28.22$ & $1.23-5.41$ & 2.463 & 29.3 & $90^{\circ}$ & $93.9^{\circ}$ & $356.9^{\circ}$ & $80.9^{\circ}$ & 9.8 & 3.6 \\
\hline $2016 / 02 / 28.29-28.44$ & $6.90-10.44$ & 2.465 & 29.2 & $90^{\circ}$ & $93.7^{\circ}$ & $356.6^{\circ}$ & $86.4^{\circ}$ & 16.1 & 12.1 \\
\hline $2016 / 03 / 14.54-14.69$ & $12.98-16.65$ & 2.577 & 15.0 & $90^{\circ}$ & $91.7^{\circ}$ & $359.7^{\circ}$ & $64.8^{\circ}$ & 8.0 & 2.5 \\
\hline $2016 / 03 / 14.72-14.90$ & $17.41-21.62$ & 2.579 & 14.5 & $90^{\circ}$ & $91.3^{\circ}$ & $359.3^{\circ}$ & $79.0^{\circ}$ & 8.0 & 6.0 \\
\hline $2016 / 04 / 12.39-12.53$ & $9.34-12.72$ & 2.784 & 124.4 & $270^{\circ}$ & $72.9^{\circ}$ & $359.0^{\circ}$ & $81.5^{\circ}$ & 15.0 & 14.0 \\
\hline $2016 / 04 / 12.56-12.73$ & $12.55-17.55$ & 2.785 & 122.2 & $270^{\circ}$ & $73.3^{\circ}$ & $360.1^{\circ}$ & $77.1^{\circ}$ & 15.9 & 15.0 \\
\hline 2016/04/12.80-12.95 & $19.19-22.76$ & 2.787 & 120.7 & $270^{\circ}$ & $74.1^{\circ}$ & $361.5^{\circ}$ & $71.8^{\circ}$ & 32.7 & 21.1 \\
\hline $2016 / 04 / 22.34-22.52$ & $8.20-12.53$ & 2.853 & 30.8 & $90^{\circ}$ & $107.1^{\circ}$ & $354.5^{\circ}$ & $123.1^{\circ}$ & 8.3 & 7.5 \\
\hline $2016 / 04 / 22.58-22.74$ & $14.00-17.65$ & 2.855 & 30.6 & $90^{\circ}$ & $109.1^{\circ}$ & $351.3^{\circ}$ & $127.5^{\circ}$ & 16.2 & 5.0 \\
\hline $2016 / 04 / 22.77-22.95$ & $18.48-22.79$ & 2.856 & 30.4 & $90^{\circ}$ & $110.7^{\circ}$ & $348.0^{\circ}$ & $131.2^{\circ}$ & 16.6 & 13.2 \\
\hline
\end{tabular}


Table A.2. Parameters derived from observations for the outgassing pattern $\left(\mathrm{H}_{2}^{18} \mathrm{O}\right)$ and temperature.

\begin{tabular}{|c|c|c|c|c|c|c|c|c|}
\hline \multirow{2}{*}{$\begin{array}{l}\text { Date } \\
\text { yyyy/mm/dd.d }\end{array}$} & \multirow{2}{*}{$\begin{array}{l}r_{\mathrm{h}}^{(a)} \\
(\mathrm{AU})\end{array}$} & \multirow{2}{*}{$\begin{array}{l}\Delta^{(b)} \\
(\mathrm{km})\end{array}$} & \multirow{2}{*}{$\begin{array}{c}v_{\exp }(c) \\
\left(\mathrm{km} \mathrm{s}^{-1}\right)\end{array}$} & \multirow{2}{*}{$\begin{array}{c}T_{\mathrm{jet}}(r)^{(d)} \\
(\mathrm{K} ; r \text { in } \mathrm{km})\end{array}$} & \multirow{2}{*}{$\begin{array}{r}T_{\text {night }}(r)^{(d)} \\
(\mathrm{K} ; r \text { in } \mathrm{km})\end{array}$} & \multirow{2}{*}{$\begin{array}{r}T(\Delta)^{(e)} \\
(\mathrm{K}) \\
\end{array}$} & \multicolumn{2}{|c|}{ Mean jet position } \\
\hline & & & & & & & PA & $\phi^{(f)}$ \\
\hline $2014 / 07 / 29.3$ & 3.645 & 1818.5 & $0.60 \pm 0.02$ & $10+260 / r$ & $20+220 / r$ & - & $331 \pm 4^{\circ}$ & $24 \pm 1^{\circ}$ \\
\hline 2014/10/13.1 & 3.190 & 18.4 & $0.60 \pm 0.04$ & $60+120 / r$ & $40+80 / r$ & - & $50 \pm 20^{\circ}$ & $90 \pm 5^{\circ}$ \\
\hline $2014 / 11 / 23.2$ & 2.920 & 30.8 & $0.60 \pm 0.02$ & $30+260 / r$ & $30+90 / r$ & $30 \pm 10$ & $70 \pm 5^{\circ}$ & $80 \pm 3^{\circ}$ \\
\hline 2014/12/13.8 & 2.777 & 19.3 & $0.60 \pm 0.02$ & $35+240 / r$ & - & $57 \pm 11$ & $75 \pm 5^{\circ}$ & $115 \pm 10^{\circ}$ \\
\hline 2015/01/01.5 & 2.643 & 28.5 & $0.64 \pm 0.02$ & $32+230 / r$ & $32+100 / r$ & $40 \pm 9$ & $65 \pm 5^{\circ}$ & $100 \pm 10^{\circ}$ \\
\hline $2015 / 02 / 16.2$ & 2.305 & 168. & $0.68 \pm 0.02$ & $19+260 / r$ & $20+160 / r$ & $19 \pm 2$ & $60 \pm 10^{\circ}$ & $88 \pm 4^{\circ}$ \\
\hline 2015/03/14.8 & 2.100 & 77.8 & $0.75 \pm 0.02$ & $25+220 / r$ & $25+190 / r$ & $24 \pm 3$ & $320 \pm 15^{\circ}$ & $37 \pm 5^{\circ}$ \\
\hline $2015 / 03 / 22.4$ & 2.042 & 78.5 & $0.72 \pm 0.06$ & $21+295 / r$ & $28+196 / r$ & $26 \pm 2$ & $69 \pm 5^{\circ}$ & $90 \pm 10^{\circ}$ \\
\hline $2015 / 05 / 15.8$ & 1.632 & 129.5 & $0.83 \pm 0.03$ & $25+280 / r$ & $21+230 / r$ & $21 \pm 6$ & $275 \pm 15^{\circ}$ & $73 \pm 5^{\circ}$ \\
\hline 2015/06/07.1 & 1.481 & 202.0 & $0.78 \pm 0.01$ & $30+290 / r$ & $25+160 / r$ & $19 \pm 2$ & $250 \pm 10^{\circ}$ & $105 \pm 15^{\circ}$ \\
\hline $2015 / 06 / 14.2$ & 1.439 & 202.0 & $0.83 \pm 0.02$ & $21+290 / r$ & $21+190 / r$ & $21 \pm 2$ & $260 \pm 20^{\circ}$ & $110 \pm 15^{\circ}$ \\
\hline $2015 / 06 / 24.7$ & 1.381 & 180.3 & $0.82 \pm 0.02$ & $30+280 / r$ & $25+200 / r$ & $23 \pm 3$ & $260 \pm 20^{\circ}$ & $112 \pm 8^{\circ}$ \\
\hline $2015 / 07 / 13.2$ & 1.300 & 153.8 & $0.84 \pm 0.02$ & $28+300 / r$ & $30+170 / r$ & $25 \pm 4$ & $246 \pm 12^{\circ}$ & $113 \pm 5^{\circ}$ \\
\hline $2015 / 07 / 23.4$ & 1.269 & 173.8 & $0.88 \pm 0.02$ & $24+366 / r / \log (9+r / 2)$ & $40+140 / r$ & $18 \pm 4$ & $237 \pm 13^{\circ}$ & $115 \pm 14^{\circ}$ \\
\hline $2015 / 07 / 30.5$ & 1.254 & 179.7 & $0.87 \pm 0.03$ & $30+350 / r$ & $50+150 / r$ & $26 \pm 2$ & $77 \pm 6^{\circ}$ & $55 \pm 13^{\circ}$ \\
\hline 2015/08/02.2 & 1.250 & 207.3 & $0.88 \pm 0.02$ & $41+221 / r$ & $48+120 / r$ & $39 \pm 2$ & $105 \pm 10^{\circ}$ & $58 \pm 4^{\circ}$ \\
\hline $2015 / 08 / 08.9$ & 1.244 & 304.8 & $0.88 \pm 0.02$ & $50+250 / r$ & $60+116 / r$ & $40 \pm 2$ & $119 \pm 3^{\circ}$ & $102 \pm 3^{\circ}$ \\
\hline 2015/08/17.7 & 1.245 & 328.5 & $0.88 \pm 0.02$ & $44+290 / r$ & $50+98 / r$ & $35 \pm 1$ & $260 \pm 9^{\circ}$ & $108 \pm 3^{\circ}$ \\
\hline 2015/08/20.3 & 1.246 & 325.9 & $0.87 \pm 0.02$ & $43+300 / r$ & $52+180 / r$ & $40 \pm 5$ & $251 \pm 11^{\circ}$ & $111 \pm 5^{\circ}$ \\
\hline $2015 / 08 / 27.6$ & 1.256 & 400.8 & $0.85 \pm 0.02$ & $43+275 / r$ & $60+116 / r$ & $42 \pm 2$ & $250 \pm 5^{\circ}$ & $54 \pm 5^{\circ}$ \\
\hline $2015 / 09 / 01.7$ & 1.267 & 424.8 & $0.86 \pm 0.02$ & $43+280 / r$ & $58+114 / r$ & $42 \pm 2$ & $95 \pm 5^{\circ}$ & $49 \pm 4^{\circ}$ \\
\hline $2015 / 09 / 08.7$ & 1.286 & 330.8 & $0.84 \pm 0.04$ & $50+280 / r$ & $60+120 / r$ & $48 \pm 2$ & $109 \pm 5^{\circ}$ & $131 \pm 2^{\circ}$ \\
\hline 2015/09/14.4 & 1.305 & 313.3 & $0.85 \pm 0.03$ & $44+275 / r$ & $51+100 / r$ & $40 \pm 1$ & $249 \pm 11^{\circ}$ & $116 \pm 6^{\circ}$ \\
\hline $2015 / 10 / 02.3$ & 1.385 & 1211. & $0.84 \pm 0.02$ & $32+270 / r$ & $48+145 / r$ & $18 \pm 2$ & $224 \pm 16^{\circ}$ & $45 \pm 11^{\circ}$ \\
\hline $2015 / 10 / 17.4$ & 1.472 & 441.6 & $0.82 \pm 0.02$ & $23+280 / r$ & $50+99 / r$ & $16 \pm 2$ & $256 \pm 5^{\circ}$ & $100 \pm 4^{\circ}$ \\
\hline $2015 / 10 / 24.6$ & 1.517 & 372.0 & $0.84 \pm 0.03$ & $25+240 / r$ & $44+100 / r$ & $19 \pm 2$ & $121 \pm 6^{\circ}$ & $90 \pm 10^{\circ}$ \\
\hline 2015/11/11.5 & 1.640 & 194.4 & $0.81 \pm 0.03$ & $16+280 / r$ & $43+140 / r$ & $17 \pm 2$ & $265 \pm 12^{\circ}$ & $100 \pm 12^{\circ}$ \\
\hline $2015 / 11 / 15.6$ & 1.669 & 152.2 & $0.84 \pm 0.02$ & $30+200 / r$ & $19+185 / r$ & $26 \pm 2$ & $131 \pm 7^{\circ}$ & $87 \pm 7^{\circ}$ \\
\hline $2015 / 11 / 22.2$ & 1.717 & 133.9 & $0.69 \pm 0.02$ & $20+300 / r$ & $30+170 / r$ & $16 \pm 2$ & $265 \pm 15^{\circ}$ & $45 \pm 15^{\circ}$ \\
\hline $2015 / 11 / 26.5$ & 1.750 & 122.4 & $0.75 \pm 0.01$ & $18+230 / r$ & $32+150 / r$ & $13 \pm 2$ & $238 \pm 6^{\circ}$ & $70 \pm 5^{\circ}$ \\
\hline $2015 / 12 / 03.4$ & 1.801 & 101.1 & $0.70 \pm 0.03$ & $30+255 / r$ & $45+110 / r$ & $24 \pm 2$ & $98 \pm 10^{\circ}$ & $123 \pm 14^{\circ}$ \\
\hline $2015 / 12 / 23.2$ & 1.952 & 88.0 & $0.70 \pm 0.02$ & $19+280 / r$ & $21+200 / r$ & $26 \pm 2$ & $81 \pm 4^{\circ}$ & $114 \pm 6^{\circ}$ \\
\hline $2015 / 12 / 28.4$ & 1.993 & 77.6 & $0.70 \pm 0.02$ & $20+230 / r$ & $49+111 / r$ & $20 \pm 2$ & $130 \pm 10^{\circ}$ & $101 \pm 3^{\circ}$ \\
\hline 2015/12/31.4 & 2.016 & 79.6 & $0.71 \pm 0.02$ & $19+210 / r$ & $38+145 / r$ & $15 \pm 2$ & $122 \pm 4^{\circ}$ & $74 \pm 13^{\circ}$ \\
\hline $2016 / 01 / 14.5$ & 2.124 & 84.7 & $0.69 \pm 0.02$ & $18+250 / r$ & $26+180 / r$ & $25 \pm 2$ & $87 \pm 10^{\circ}$ & $108 \pm 9^{\circ}$ \\
\hline $2016 / 01 / 25.4$ & 2.208 & 71.1 & $0.69 \pm 0.03$ & $17+270 / r$ & $38+140 / r$ & $14 \pm 3$ & $220 \pm 30^{\circ}$ & $34 \pm 19^{\circ}$ \\
\hline $2016 / 02 / 28.2$ & 2.463 & 29.3 & $0.60 \pm 0.03$ & $30+230 / r$ & $45+140 / r$ & $42 \pm 4$ & $127 \pm 15^{\circ}$ & $85 \pm 5^{\circ}$ \\
\hline $2016 / 03 / 14.7$ & 2.578 & 14.7 & $0.60 \pm 0.03$ & $33+240 / r$ & $40+140 / r$ & $23 \pm 6$ & $115 \pm 17^{\circ}$ & $104 \pm 3^{\circ}$ \\
\hline $2016 / 04 / 12.7$ & 2.785 & 122.4 & $0.58 \pm 0.03$ & $30+220 / r$ & $30+130 / r$ & $30 \pm 3$ & $245 \pm 45^{\circ}$ & $77 \pm 8^{\circ}$ \\
\hline $2016 / 04 / 22.7$ & 2.855 & 30.6 & $0.56 \pm 0.03$ & $30+270 / r$ & $24+160 / r$ & $24 \pm 3$ & $85 \pm 20^{\circ}$ & $95 \pm 10^{\circ}$ \\
\hline
\end{tabular}

Notes. ${ }^{(a)}$ Heliocentric distance. ${ }^{(b)}$ Distance Rosetta-comet. ${ }^{(c)}$ Average based on fit to nadir observations for the $\mathrm{H}_{2}^{18} \mathrm{O}$, $\mathrm{H}_{2}^{17} \mathrm{O}$, $\mathrm{CH}_{3} \mathrm{OH}\left(3_{-2}-2_{-1} E\right), \quad \mathrm{CH}_{3} \mathrm{OH}\left(12_{-1}-11_{-1} E\right)$, and $\mathrm{NH}_{3}$ lines. ${ }^{(d)}$ Temperature profile constrained by the $\mathrm{H}_{2}^{16} \mathrm{O}$ peak temperature and $T_{\text {rot }}\left(\mathrm{CH}_{3} \mathrm{OH}\left(3_{-2}-2_{-1} E\right), \mathrm{CH}_{3} \mathrm{OH}\left(12_{-1}-11_{-1} E\right)\right.$ ) (see text). ${ }^{(e)}$ Value of $T_{\mathrm{mb}}\left(\mathrm{H}_{2}^{16} \mathrm{O}\right)\left(-v_{\text {exp }}\right)$ in emission and absorption. ${ }^{(f)}$ Mean jet colatitude (angle between line of sight and direction of peak outgassing). 
Table A.3. 2D Gaussian fit to the jet ${ }^{(a)}$ outgassing pattern and derived production rate.

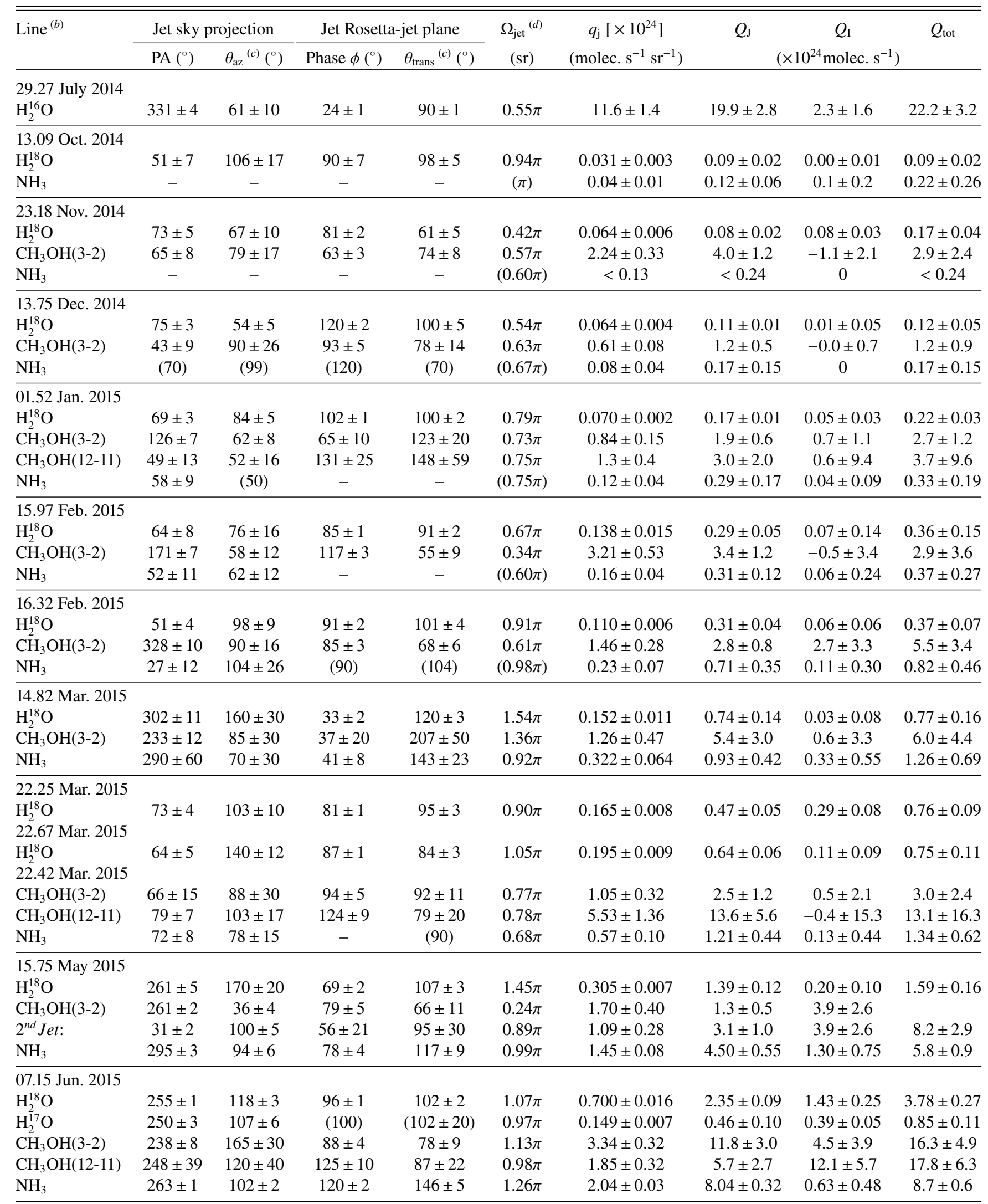

Notes. ${ }^{(a)}$ The geometrical characteristics of the jet are provided in Cols. 2-5: values in parentheses are assumed (inferred from other molecular lines). ${ }^{(b)} \mathrm{CH}_{3} \mathrm{OH}(3-2)=\mathrm{CH}_{3} \mathrm{OH}\left(3_{-2}-2_{-1} E\right) ; \mathrm{CH}_{3} \mathrm{OH}(8-7)=\mathrm{CH}_{3} \mathrm{OH}\left(8_{+1}-7_{0} E\right) ; \mathrm{CH}_{3} \mathrm{OH}(12-11)=\mathrm{CH}_{3} \mathrm{OH}\left(12_{-1}-11_{-1} E\right)$. ${ }^{(c)} \mathrm{FWHM}$ of the Gaussian fit in degrees. ${ }^{(d)}$ Solid angle of the jet (integral over $4 \pi$ of the 2 D Gaussian profile with the mean FWHM $\theta_{\mathrm{j}}$ taken as geometric average of Cols. 3 and 5 value). The uncertainty on $\Omega_{\text {jet }}$ due to uncertainties on the FWHMs of the jet is taken into account to compute uncertainties on the gas production in the jet (Cols. 8 and 10). 
Table A.3. continued.

\begin{tabular}{|c|c|c|c|c|c|c|c|c|c|}
\hline \multirow[t]{2}{*}{ Line $^{(b)}$} & \multicolumn{2}{|c|}{ Jet sky projection } & \multicolumn{2}{|c|}{ Jet Rosetta-jet plane } & \multirow{2}{*}{$\begin{array}{c}\Omega_{\mathrm{jet}}(d) \\
(\mathrm{sr}) \\
\end{array}$} & \multirow{2}{*}{$\begin{array}{c}q_{\mathrm{j}}\left[\times 10^{24}\right] \\
\left(\text { molec. } \mathrm{s}^{-1} \mathrm{sr}^{-1}\right)\end{array}$} & \multirow[t]{2}{*}{$Q_{\mathrm{J}}$} & \multirow{2}{*}{$\begin{array}{c}Q_{\mathrm{I}} \\
\left(\times 10^{24} \text { molec. } \mathrm{s}^{-1}\right)\end{array}$} & \multirow[t]{2}{*}{$Q_{\text {tot }}$} \\
\hline & $\mathrm{PA}\left({ }^{\circ}\right)$ & $\theta_{\mathrm{az}}{ }^{(c)}\left(^{\circ}\right)$ & Phase $\phi\left(^{\circ}\right)$ & $\theta_{\text {trans }}{ }^{(c)}\left({ }^{\circ}\right)$ & & & & & \\
\hline \multicolumn{10}{|l|}{ 14.09 Jun. 2015} \\
\hline $\begin{array}{l}\mathrm{H}_{2}^{18} \mathrm{O} \\
14.19 \text { Jun. } 2015\end{array}$ & $240 \pm 4$ & $209 \pm 30$ & $103 \pm 2$ & $120 \pm 3$ & $1.79 \pi$ & $0.765 \pm 0.028$ & $4.30 \pm 0.35$ & $0.39 \pm 0.16$ & $4.69 \pm 0.38$ \\
\hline $\begin{array}{l}\mathrm{H}_{2}^{18} \mathrm{O} \\
14.32 \text { Jun. } 2015\end{array}$ & $273 \pm 3$ & $138 \pm 8$ & $96 \pm 1$ & $134 \pm 3$ & $1.46 \pi$ & $0.686 \pm 0.010$ & $3.15 \pm 0.17$ & $0.58 \pm 0.23$ & $3.73 \pm 0.29$ \\
\hline $\begin{array}{l}\mathrm{H}_{2}^{18} \mathrm{O} \\
14.41 \text { Jun. } 2015\end{array}$ & $273 \pm 3$ & $149 \pm 8$ & $114 \pm 2$ & $141 \pm 4$ & $1.60 \pi$ & $0.794 \pm 0.028$ & $3.98 \pm 0.25$ & $0.36 \pm 0.23$ & $4.34 \pm 0.34$ \\
\hline $\begin{array}{l}\mathrm{H}_{2}^{18} \mathrm{O} \\
14.24 \text { Jun. } 2015\end{array}$ & $265 \pm 3$ & $161 \pm 18$ & $127 \pm 3$ & $147 \pm 4$ & $1.72 \pi$ & $0.538 \pm 0.023$ & $2.92 \pm 0.25$ & $0.73 \pm 0.24$ & $3.65 \pm 0.35$ \\
\hline $\mathrm{CH}_{3} \mathrm{OH}(3-2)$ & $240 \pm 35$ & $190 \pm 60$ & $102 \pm 4$ & $105 \pm 10$ & $1.54 \pi$ & $4.13 \pm 0.63$ & $20.0 \pm 5.7$ & $1.3 \pm 5.9$ & $21.3 \pm 8.2$ \\
\hline $\mathrm{CH}_{3} \mathrm{OH}(12-11)$ & $296 \pm 35$ & $124 \pm 90$ & $190 \pm 40$ & $188 \pm 24$ & $1.70 \pi$ & $6.46 \pm 1$ & $34.5 \pm 15.7$ & $-8.8 \pm 13.1$ & $25.7 \pm 20.4$ \\
\hline $\mathrm{NH}_{3}$ & $283 \pm 3$ & $143 \pm 8$ & $106 \pm 2$ & $130 \pm 4$ & $1.47 \pi$ & $1.97 \pm 0.07$ & $9.08 \pm 0.58$ & $-0.21 \pm 0.66$ & $8.9 \pm 0.9$ \\
\hline $\mathrm{CO}$ & $200 \pm 30$ & $80 \pm 30$ & $87 \pm 16$ & $163 \pm 32$ & $1.14 \pi$ & $7.8 \pm 1.4$ & $28 \pm 14$ & - & $28 \pm 14$ \\
\hline \multicolumn{10}{|l|}{24.52 Jun. 2015} \\
\hline $\begin{array}{l}\mathrm{H}_{2}^{18} \mathrm{O} \\
24.95 \text { Jun. } 2015\end{array}$ & $268 \pm 2$ & $132 \pm 4$ & $107 \pm 1$ & $142 \pm 2$ & $1.48 \pi$ & $0.788 \pm 0.015$ & $3.67 \pm 0.13$ & $1.09 \pm 0.19$ & $4.76 \pm 0.23$ \\
\hline $\begin{array}{l}\mathrm{H}_{2}^{18} \mathrm{O} \\
24.74 \text { Jun. } 2015\end{array}$ & $247 \pm 2$ & $129 \pm 5$ & $104 \pm 1$ & $128 \pm 2$ & $1.35 \pi$ & $0.890 \pm 0.022$ & $3.78 \pm 0.17$ & $1.37 \pm 0.16$ & $5.15 \pm 0.23$ \\
\hline $\mathrm{CH}_{3} \mathrm{OH}$ & $282 \pm 4$ & $58 \pm 7$ & $120 \pm 4$ & $121 \pm 8$ & $0.68 \pi$ & $5.95 \pm 0.58$ & $12.7 \pm 2.4$ & & \\
\hline $2^{\text {nd }} \mathrm{Jet}:$ & $140 \pm 6$ & $83 \pm 12$ & $102 \pm 4$ & $105 \pm 10$ & $0.60 \pi$ & $6.73 \pm$ & $12.7 \pm 3.0$ & $2.1 \pm 4.9$ & $27.5 \pm 6.2$ \\
\hline $\mathrm{CH}_{3} \mathrm{OH}(12-11)$ & $224 \pm 28$ & $70 \pm 9$ & $117 \pm 20$ & $128 \pm 38$ & $0.84 \pi$ & $7.60 \pm 1.70$ & $20.1 \pm 7.6$ & & \\
\hline $2^{\text {nd }} \mathrm{Jet}:$ & (150) & (60) & & (60) & $0.37 \pi$ & $2.0 \pm 2.0$ & $2.4 \pm 2.4$ & $16.3 \pm 22.6$ & $39 . \pm 24$ \\
\hline $\mathrm{NH}_{3}$ & $281 \pm 1$ & $118 \pm 3$ & $118 \pm 2$ & $127 \pm 3$ & $1.26 \pi$ & $2.27 \pm 0.05$ & $9.0 \pm 0.4$ & $1.9 \pm 0.6$ & $10.9 \pm 0.7$ \\
\hline $\mathrm{CO}$ & $(150-270)$ & (122) & & (122) & $(1.25 \pi)$ & $-0.92 \pm 1.67$ & $-3.6 \pm 6.6$ & - & $<19.7$ \\
\hline \multicolumn{10}{|l|}{13.20 July 2015} \\
\hline $\mathrm{H}_{2}^{18} \mathrm{O}$ & $246 \pm 2$ & $128 \pm 4$ & $109 \pm 1$ & $126 \pm 2$ & $1.33 \pi$ & $1.410 \pm 0.026$ & $5.88 \pm 0.21$ & $1.41 \pm 0.29$ & $7.29 \pm 0.36$ \\
\hline $\mathrm{CH}_{3} \mathrm{OH}(3-2)$ & $34 \pm 6$ & \pm 15 & $110 \pm 6$ & $119 \pm 13$ & $1.09 \pi$ & 9.72 & $33.2 \pm$ & $8.3 \pm$ & $41.6 \pm 9.6$ \\
\hline $\mathrm{CH}_{3} \mathrm{OH}(12-11)$ & $10 \pm 9$ & $82 \pm 15$ & $119 \pm 9$ & $98 \pm 17$ & $0.76 \pi$ & $15.5 \pm 2.7$ & $37.0 \pm 12.9$ & $8.2 \pm 32.8$ & $45.2 \pm 35.2$ \\
\hline $\mathrm{NH}_{3}$ & $258 \pm 2$ & $137 \pm 6$ & $115 \pm 2$ & $137 \pm 4$ & $1.48 \pi$ & $3.94 \pm 0.12$ & $18.4 \pm 1.1$ & $-0.2 \pm 1.2$ & $18.2 \pm 1.7$ \\
\hline \multicolumn{10}{|l|}{23.38 July 2015} \\
\hline $\mathrm{H}_{2}^{18} \mathrm{O}$ & $237 \pm 1$ & $127 \pm 2$ & $107 \pm 1$ & $132 \pm 1$ & $1.37 \pi$ & $2.400 \pm 0.032$ & $10.31 \pm 0.22$ & $0.97 \pm 0.38$ & $11.27 \pm 0.44$ \\
\hline $\mathrm{CH}_{3} \mathrm{OH}(3-2)$ & $222 \pm 4$ & $113 \pm 11$ & $98 \pm 3$ & \pm 9 & $0.85 \pi$ & & $42.3 \pm 7.4$ & $5.0 \pm 9.3$ & $47.3 \pm 11.9$ \\
\hline $\mathrm{CH}_{3} \mathrm{OH}(12-11)$ & $229 \pm 10$ & $134 \pm 28$ & $121 \pm 7$ & $80 \pm 13$ & $0.97 \pi$ & $25.64 \pm 4.33$ & $78.1 \pm 26.3$ & $-7.0 \pm 41.8$ & $71.1 \pm 49.4$ \\
\hline $\mathrm{NH}_{3}$ & $252 \pm 1$ & $145 \pm 4$ & $129 \pm 2$ & & $1.33 \pi$ & $5.97 \pm 0.12$ & $24.8 \pm 1.1$ & & $27.5 \pm 1.5$ \\
\hline \multicolumn{10}{|l|}{30.38 July 2015} \\
\hline $\begin{array}{l}\mathrm{H}_{2}^{18} \mathrm{O} \\
30.57 \text { July } 2015\end{array}$ & $75 \pm 1$ & $142 \pm 4$ & $60 \pm 1$ & $100 \pm 2$ & $1.21 \pi$ & $2.569 \pm 0.044$ & $9.78 \pm 0.35$ & $0.88 \pm 0.09$ & $10.66 \pm 0.36$ \\
\hline $\begin{array}{l}\mathrm{H}_{2}^{18} \mathrm{O} \\
30.81 \text { July } 2015\end{array}$ & $75 \pm 1$ & $119 \pm 4$ & $54 \pm 2$ & $128 \pm 2$ & $1.28 \pi$ & $2.558 \pm 0.065$ & $10.25 \pm 0.44$ & $1.16 \pm 0.13$ & $11.41 \pm 0.46$ \\
\hline $\begin{array}{l}\mathrm{H}_{2}^{18} \mathrm{O} \\
30.59 \text { July } 2015\end{array}$ & $85 \pm 2$ & $157 \pm 10$ & $55 \pm 3$ & $124 \pm 2$ & $1.52 \pi$ & $2.168 \pm 0.041$ & $10.34 \pm 0.58$ & $0.68 \pm 0.11$ & $11.02 \pm 0.59$ \\
\hline $\mathrm{H}_{2}^{17} \mathrm{O}$ & $74 \pm 2$ & $120 \pm 11$ & & $(120)$ & $1.23 \pi$ & & $1.32 \pm 0.19$ & $0.62 \pm 0.08$ & $1.94 \pm 0.21$ \\
\hline $\mathrm{CH}_{3} \mathrm{OH}(3-2)$ & $76 \pm 4$ & $118 \pm 15$ & $34 \pm 1$ & & $1.20 \pi$ & $16.87 \pm 0$ & $63.5 \pm 9.1$ & & $61.8 \pm 9.9$ \\
\hline $\mathrm{CH}_{3} \mathrm{OH}(8-7)$ & - & - & $75 \pm 8$ & $128 \pm 19$ & $1.1 \pi$ & $20.38 \pm 2.66$ & $70.4 \pm 11.2$ & $6.3 \pm 37.0$ & $76.7 \pm 38.7$ \\
\hline $\mathrm{CH}_{3} \mathrm{OH}(12-11)$ & $65 \pm 7$ & $75 \pm 11$ & $46 \pm 3$ & $105 \pm 7$ & $0.76 \pi$ & $32.60 \pm 2.46$ & $77.7 \pm 15.0$ & $-13.7 \pm 8.2$ & $64.0 \pm 27.2$ \\
\hline $\mathrm{NH}_{3}$ & $80 \pm 1$ & $134 \pm 4$ & $69 \pm 1$ & $127 \pm 3$ & $1.39 \pi$ & $5.64 \pm 0.11$ & $24.6 \pm 1.0$ & $0.1 \pm 0.4$ & $24.7 \pm 1.1$ \\
\hline $\mathrm{CO}$ & $81 \pm 6$ & $68 \pm 47$ & $67 \pm 4$ & $123 \pm 41$ & $0.85 \pi$ & $11.59 \pm 5.07$ & $30.9 \pm 27.3$ & $-11.3 \pm$ & $19.6 \pm 31.1$ \\
\hline \multicolumn{10}{|l|}{ 02.13 Aug. 2015} \\
\hline $\begin{array}{l}\mathrm{H}_{2}^{18} \mathrm{O} \\
02.36 \text { Aug. } 2015\end{array}$ & $107 \pm 1$ & $135 \pm 4$ & $54 \pm 1$ & $102 \pm 1$ & $1.19 \pi$ & $3.02 \pm 0.08$ & $11.3 \pm 0.4$ & $0.9 \pm 0.3$ & $12.2 \pm 0.5$ \\
\hline $\begin{array}{l}\mathrm{H}_{2}^{18} \mathrm{O} \\
02.24 \text { Aug. } 2015\end{array}$ & $108 \pm 2$ & $134 \pm 5$ & $62 \pm 1$ & $119 \pm 1$ & $1.32 \pi$ & $2.58 \pm 0.06$ & $10.7 \pm 0.4$ & $0.6 \pm 0.4$ & $11.3 \pm 0.5$ \\
\hline $\mathrm{H}_{2}^{17} \mathrm{O}$ & $121 \pm 5$ & $136 \pm 14$ & & - & & & $1.77 \pm 0.28$ & $0.08 \pm 0.46$ & $1.85 \pm 0.54$ \\
\hline $\mathrm{CH}_{3} \mathrm{OH}(3-2)$ & $118 \pm 6$ & $137 \pm 19$ & $59 \pm 2$ & $105 \pm 3$ & $1.22 \pi$ & $18.09 \pm 1.05$ & $69.4 \pm 7.9$ & $-0.6 \pm 9.7$ & $68.8 \pm 12.5$ \\
\hline $\mathrm{CH}_{3} \mathrm{OH}(8-7)$ & $134 \pm 10$ & $107 \pm 21$ & 60 & - & $1.03 \pi$ & $5.17 \pm 2.47$ & $16.7 \pm 9.4$ & $35.8 \pm 46.5$ & $52.5 \pm 47.4$ \\
\hline $\mathrm{CH}_{3} \mathrm{OH}(12-11)$ & $100 \pm 6$ & $84 \pm 14$ & $56 \pm 4$ & $92 \pm 8$ & $0.74 \pi$ & $26.00 \pm 2.48$ & $60.4 \pm 13.4$ & $16.7 \pm 30.8$ & $77.1 \pm 33.6$ \\
\hline
\end{tabular}


Table A.3. continued.

\begin{tabular}{|c|c|c|c|c|c|c|c|c|c|}
\hline \multirow[t]{2}{*}{ Line $^{(b)}$} & \multicolumn{2}{|c|}{ Jet sky projection } & \multicolumn{2}{|c|}{ Jet Rosetta-jet plane } & \multirow{2}{*}{$\begin{array}{c}\Omega_{\mathrm{jet}}(d) \\
(\mathrm{sr}) \\
\end{array}$} & \multirow{2}{*}{$\begin{array}{c}q_{\mathrm{j}}\left[\times 10^{24}\right] \\
\left(\text { molec. } \mathrm{s}^{-1} \mathrm{sr}^{-1}\right)\end{array}$} & \multirow[t]{2}{*}{$Q_{\mathrm{J}}$} & \multirow{2}{*}{$\begin{array}{c}Q_{\mathrm{I}} \\
\left.\times 10^{24} \text { molec. } \mathrm{s}^{-1}\right) \\
\end{array}$} & \multirow[t]{2}{*}{$Q_{\text {tot }}$} \\
\hline & $\mathrm{PA}\left({ }^{\circ}\right)$ & $\theta_{\mathrm{az}}{ }^{(c)}\left(^{\circ}\right)$ & Phase $\phi\left(^{\circ}\right)$ & $\theta_{\text {trans }}(c)\left({ }^{\circ}\right)$ & & & & & \\
\hline $\mathrm{NH}_{3}$ & $95 \pm 2$ & $129 \pm 4$ & $57 \pm 2$ & $130 \pm 2$ & $1.37 \pi$ & $5.60 \pm 0.12$ & $24.07 \pm 0.99$ & $1.13 \pm 1.11$ & $25.2 \pm 1.5$ \\
\hline $\mathrm{CO}$ & $197 \pm 20$ & $177 \pm 24$ & - & $(100)$ & $1.38 \pi$ & $8.11 \pm 1.19$ & $35.2 \pm 11.2$ & $-4.5 \pm 21.4$ & $30.7 \pm 24.2$ \\
\hline \multicolumn{10}{|c|}{08.94 Aug. 2015} \\
\hline $\begin{array}{l}\mathrm{H}_{2}^{18} \mathrm{O} \\
09.13 \text { Aug. } 2015\end{array}$ & $115 \pm 1$ & $150 \pm 4$ & $100 \pm 2$ & $90 \pm 2$ & $1.17 \pi$ & $3.16 \pm 0.06$ & $11.6 \pm 0.5$ & $0.8 \pm 0.1$ & $12.4 \pm 0.5$ \\
\hline $\begin{array}{l}\mathrm{H}_{2}^{18} \mathrm{O} \\
09.04 \text { Aug. } 2015\end{array}$ & $120 \pm 2$ & $134 \pm 3$ & $94 \pm 2$ & $127 \pm 3$ & $1.39 \pi$ & $2.98 \pm 0.04$ & $13.0 \pm 0.5$ & $1.0 \pm 0.1$ & $14.0 \pm 0.5$ \\
\hline $\mathrm{H}_{2}^{17} \mathrm{O}$ & $130 \pm 7$ & $125 \pm 10$ & $(100)$ & $(105)$ & $1.14 \pi$ & $0.52 \pm 0.04$ & $1.87 \pm 0.29$ & $0.29 \pm 0.10$ & $2.16 \pm 0.31$ \\
\hline $\mathrm{CH}_{3} \mathrm{OH}(3-2)$ & $124 \pm 8$ & $105 \pm 10$ & $111 \pm 3$ & $101 \pm 8$ & $0.96 \pi$ & $27.8 \pm 1.8$ & $83.6 \pm 13.2$ & $0.9 \pm 5.2$ & $84.5 \pm 14.2$ \\
\hline $\mathrm{CH}_{3} \mathrm{OH}(8-7)$ & $124 \pm 30$ & $95 \pm 29$ & $104 \pm 11$ & $90 \pm 20$ & $0.80 \pi$ & $31.3 \pm 8.0$ & $78.8 \pm 39.9$ & $37.4 \pm 18.0$ & $116.2 \pm 43.8$ \\
\hline $\mathrm{CH}_{3} \mathrm{OH}(12-11)$ & $132 \pm 22$ & $89 \pm 15$ & $109 \pm 9$ & $79 \pm 15$ & $0.68 \pi$ & $29.2 \pm 5.4$ & $62.2 \pm 22.5$ & $23.6 \pm 14.4$ & $85.8 \pm 26.7$ \\
\hline $\mathrm{NH}_{3}$ & $112 \pm 2$ & $146 \pm 5$ & $98 \pm 3$ & $129 \pm 6$ & $1.48 \pi$ & $5.76 \pm 0.18$ & $26.8 \pm 1.7$ & $-0.9 \pm 0.5$ & $25.8 \pm 1.8$ \\
\hline $\mathrm{CO}$ & - & - & - & - & $1.1 \pi$ & $7.3 \pm 2.3$ & $25 \pm 8$ & - & - \\
\hline \multicolumn{10}{|l|}{17.61 Aug. 2015} \\
\hline $\begin{array}{l}\mathrm{H}_{2}^{18} \mathrm{O} \\
17.79 \text { Aug. } 2015\end{array}$ & $256 \pm 1$ & $139 \pm 3$ & $108 \pm 1$ & $109 \pm 1$ & $1.27 \pi$ & $3.51 \pm 0.05$ & $14.0 \pm 0.4$ & $0.5 \pm 0.3$ & $14.6 \pm 0.5$ \\
\hline $\begin{array}{l}\mathrm{H}_{2}^{18} \mathrm{O} \\
17.70 \text { Aug. } 2015\end{array}$ & $267 \pm 1$ & $139 \pm 3$ & $111 \pm 1$ & $114 \pm 1$ & $1.31 \pi$ & $3.39 \pm 0.05$ & $14.0 \pm 0.4$ & $0.1 \pm 0.3$ & $14.1 \pm 0.5$ \\
\hline $\mathrm{H}_{2}^{17} \mathrm{O}$ & $249 \pm 6$ & $115 \pm 18$ & $(110)$ & $(114)$ & $1.15 \pi$ & $0.46 \pm 0.06$ & $1.66 \pm 0.43$ & $0.30 \pm 0.43$ & $1.96 \pm 0.61$ \\
\hline $\mathrm{CH}_{3} \mathrm{OH}(3-2)$ & $251 \pm 4$ & $22 \pm 10$ & $113 \pm 2$ & $93 \pm 4$ & $1.03 \pi$ & $30.80 \pm 1.34$ & $99.5 \pm 10.4$ & $-4.0 \pm$ & $95.5 \pm 17.3$ \\
\hline $\mathrm{CH}_{3} \mathrm{OH}(8-7)$ & $276 \pm 16$ & $87 \pm 40$ & - & $(87)$ & $0.78 \pi$ & $27.91 \pm 10.37$ & $68.4 \pm 54.5$ & $-1.1 \pm 1$ & $67.3 \pm 143.8$ \\
\hline $\mathrm{CH}_{3} \mathrm{OH}(12-11)$ & $258 \pm 3$ & $83 \pm 8$ & $116 \pm 3$ & $74 \pm 6$ & $0.61 \pi$ & $42.15 \pm 2.83$ & $80.8 \pm 14.3$ & $31.4 \pm 3$ & $112.2 \pm 35.5$ \\
\hline $\mathrm{NH}_{3}$ & $269 \pm 2$ & $138 \pm 4$ & $108 \pm 3$ & $125 \pm 6$ & $1.40 \pi$ & 0.25 & $33.6 \pm 2.2$ & $1.3 \pm$ & $34.9 \pm 3.4$ \\
\hline $\mathrm{CO}$ & $261 \pm 6$ & $91 \pm 22$ & - & $(91)$ & $0.79 \pi$ & 8.9 & $31.4 \pm 25.3$ & $6.9 \pm$ & $38.3 \pm 87.5$ \\
\hline \multicolumn{10}{|l|}{20.15 Aug. 2015} \\
\hline $\begin{array}{l}\mathrm{H}_{2}^{18} \mathrm{O} \\
20.37 \text { Aug. } 2015\end{array}$ & $246 \pm 1$ & $126 \pm 2$ & $107 \pm 1$ & $109 \pm 1$ & $1.18 \pi$ & $3.19 \pm 0.03$ & $11.8 \pm 0.2$ & $2.1 \pm 0.3$ & $13.9 \pm 0.3$ \\
\hline $\begin{array}{l}\mathrm{H}_{2}^{18} \mathrm{O} \\
20.25 \text { Aug. } 2015\end{array}$ & $251 \pm 1$ & $151 \pm 3$ & $111 \pm 1$ & $110 \pm 1$ & $1.37 \pi$ & $3.44 \pm 0.04$ & $14.8 \pm 0.3$ & $0.8 \pm 0.3$ & $15.6 \pm 0.4$ \\
\hline $\mathrm{H}_{2}^{17} \mathrm{O}$ & $245 \pm 5$ & $126 \pm 13$ & $(110)$ & $(110)$ & $1.19 \pi$ & $0.57 \pm 0.05$ & $2.15 \pm 0.35$ & $-0.03 \pm 0.41$ & $2.12 \pm 0.54$ \\
\hline $\mathrm{CH}_{3} \mathrm{OH}(3-2)$ & $240 \pm 4$ & $112 \pm 10$ & $106 \pm 1$ & $94 \pm 4$ & $0.96 \pi$ & 1.33 & $80.7 \pm 9.0$ & 4.4 & $85.1 \pm 13.2$ \\
\hline $\mathrm{CH}_{3} \mathrm{OH}(8-7)$ & $232 \pm 9$ & $100 \pm 24$ & $115 \pm 6$ & $77 \pm 12$ & $0.74 \pi$ & $33.54 \pm 4.33$ & $78.0 \pm 27.2$ & $27.1 \pm 44.0$ & $105.1 \pm 51.7$ \\
\hline $\mathrm{CH}_{3} \mathrm{OH}(12-11)$ & $241 \pm 7$ & $82 \pm 14$ & $118 \pm 3$ & $82 \pm 7$ & $0.65 \pi$ & $36.86 \pm 3.80$ & $75.6 \pm 17.5$ & $27.1 \pm 44.3$ & $102.7 \pm 47.6$ \\
\hline $\mathrm{NH}_{3}$ & $264 \pm 2$ & $152 \pm 5$ & $115 \pm 2$ & $130 \pm 4$ & $1.54 \pi$ & $5.47 \pm 0.14$ & $26.5 \pm 1.3$ & $1.2 \pm 2.3$ & $27.7 \pm 2.7$ \\
\hline $\mathrm{CO}$ & $296 \pm 10$ & $60 \pm 25$ & $160 \pm 16$ & $132 \pm 26$ & $0.76 \pi$ & $18.3 \pm 4.1$ & $43.7 \pm 22.9$ & $0.4 \pm 70.0$ & $44.1 \pm 73.6$ \\
\hline \multicolumn{10}{|l|}{27.39 Aug. 2015} \\
\hline $\begin{array}{l}\mathrm{H}_{2}^{18} \mathrm{O} \\
27.58 \text { Aug. } 2015\end{array}$ & $255 \pm 1$ & $171 \pm 10$ & $49 \pm 1$ & $110 \pm 1$ & $1.48 \pi$ & $3.01 \pm 0.05$ & $14.0 \pm 0.7$ & $1.0 \pm 0.3$ & $15.0 \pm 0.8$ \\
\hline $\begin{array}{l}\mathrm{H}_{2}^{18} \mathrm{O} \\
27.80 \text { Aug. } 2015\end{array}$ & $246 \pm 1$ & $114 \pm 2$ & $51 \pm 1$ & $115 \pm 1$ & $1.14 \pi$ & $3.84 \pm 0.06$ & $13.7 \pm 0.3$ & $1.8 \pm 0.4$ & $15.5 \pm 0.5$ \\
\hline $\begin{array}{l}\mathrm{H}_{2}^{18} \mathrm{O} \\
27.60 \text { Aug. } 2015\end{array}$ & $251 \pm 1$ & $92 \pm 2$ & $57 \pm 1$ & $131 \pm 1$ & $1.07 \pi$ & $4.12 \pm 0.06$ & $13.8 \pm 0.4$ & $1.9 \pm 0.4$ & $15.8 \pm 0.5$ \\
\hline $\mathrm{H}_{2}^{17} \mathrm{O}$ & $248 \pm 6$ & $142 \pm 20$ & $(52)$ & $(115 \pm 10)$ & $1.35 \pi$ & $0.51 \pm 0.04$ & $2.14 \pm 0.38$ & $-0.06 \pm 0.50$ & $2.08 \pm 0.63$ \\
\hline $\mathrm{CH}_{3} \mathrm{OH}(3-2)$ & $248 \pm 3$ & $92 \pm 10$ & $45 \pm 1$ & $118 \pm 3$ & $0.98 \pi$ & $32.3 \pm 1.1$ & $99.5 \pm 9.0$ & $4.9 \pm 12.7$ & $104.4 \pm 15.5$ \\
\hline $\mathrm{CH}_{3} \mathrm{OH}(12-11)$ & $254 \pm 7$ & $83 \pm 18$ & $55 \pm 4$ & $99 \pm 8$ & $0.79 \pi$ & $43.7 \pm 4.4$ & $108.5 \pm 27.0$ & $-5.0 \pm 22.9$ & $103.5 \pm 35.4$ \\
\hline $\mathrm{NH}_{3}$ & $250 \pm 1$ & $121 \pm 3$ & $63 \pm 1$ & $142 \pm 3$ & $1.39 \pi$ & $5.50 \pm 0.10$ & $24.1 \pm 0.9$ & $3.3 \pm 1.9$ & $27.3 \pm 2.1$ \\
\hline $\mathrm{CO}$ & - & $(116)$ & $38 \pm 17$ & $116 \pm 39$ & $1.16 \pi$ & $16.4 \pm 4.3$ & $59.8 \pm 33.7$ & $-3.0 \pm 47.0$ & $56.8 \pm 57.8$ \\
\hline 01.64 Sept. 2015 & & & & & & & & & \\
\hline $\begin{array}{l}\mathrm{H}_{2}^{18} \mathrm{O} \\
01.87 \text { Sept. } 2015\end{array}$ & $90 \pm 1$ & $135 \pm 4$ & $49 \pm 1$ & $121 \pm 1$ & $1.34 \pi$ & $3.29 \pm 0.07$ & $13.8 \pm 0.4$ & $1.2 \pm 0.3$ & $15.0 \pm 0.6$ \\
\hline $\begin{array}{l}\mathrm{H}_{2}^{18} \mathrm{O} \\
01.75 \text { Sept. } 2015\end{array}$ & $99 \pm 1$ & $126 \pm 3$ & $50 \pm 0$ & $121 \pm 1$ & $1.28 \pi$ & $3.47 \pm 0.05$ & $13.9 \pm 0.4$ & $1.2 \pm 0.2$ & $15.1 \pm 0.4$ \\
\hline $\mathrm{H}_{2}^{17} \mathrm{O}$ & $93 \pm 4$ & $122 \pm 12$ & $(50)$ & $(120 \pm 10)$ & $1.24 \pi$ & & $2.48 \pm 0.37$ & $0.19 \pm 0.33$ & $2.67 \pm 0.50$ \\
\hline $\mathrm{CH}_{3} \mathrm{OH}(3-2)$ & $114 \pm 4$ & $114 \pm 12$ & $43 \pm 2$ & $115 \pm 4$ & $1.14 \pi$ & $27.31 \pm 1.13$ & $97.9 \pm 11.3$ & $12.1 \pm 11.4$ & $110.0 \pm 16.1$ \\
\hline $\mathrm{CH}_{3} \mathrm{OH}(8-7)$ & $113 \pm 10$ & $95 \pm 21$ & $62 \pm 8$ & $122 \pm 15$ & $1.04 \pi$ & $32.88 \pm 5.07$ & $106.9 \pm 33.6$ & $-15.4 \pm 44.3$ & $91.5 \pm 55.6$ \\
\hline $\mathrm{CH}_{3} \mathrm{OH}(12-11)$ & $82 \pm 8$ & $133 \pm 43$ & $46 \pm 4$ & $121 \pm 10$ & $1.32 \pi$ & $31.48 \pm 2.74$ & $130.5 \pm 40.2$ & $-23.2 \pm 34.6$ & $107.3 \pm 53.0$ \\
\hline
\end{tabular}


Table A.3. continued.

\begin{tabular}{|c|c|c|c|c|c|c|c|c|c|}
\hline \multirow[t]{2}{*}{$\operatorname{Line}^{(b)}$} & \multicolumn{2}{|c|}{ Jet sky projection } & \multicolumn{2}{|c|}{ Jet Rosetta-jet plane } & \multirow{2}{*}{$\begin{array}{c}\Omega_{\text {jet }}{ }^{(d)} \\
(\mathrm{sr}) \\
\end{array}$} & \multirow{2}{*}{$\begin{array}{c}q_{\mathrm{j}}\left[\times 10^{24}\right] \\
\left(\text { molec. } \mathrm{s}^{-1} \mathrm{sr}^{-1}\right)\end{array}$} & \multirow[t]{2}{*}{$Q_{\mathrm{J}}$} & \multirow{2}{*}{$\begin{array}{c}Q_{\mathrm{I}} \\
\left.\times 10^{24} \text { molec. } \mathrm{s}^{-1}\right) \\
\end{array}$} & \multirow[t]{2}{*}{$Q_{\text {tot }}$} \\
\hline & $\mathrm{PA}\left({ }^{\circ}\right)$ & $\theta_{\mathrm{az}}{ }^{(c)}\left(^{\circ}\right)$ & Phase $\phi\left(^{\circ}\right)$ & $\theta_{\text {trans }}(c)\left({ }^{\circ}\right)$ & & & & & \\
\hline $\begin{array}{l}\mathrm{NH}_{3} \\
\mathrm{CO}\end{array}$ & $\begin{array}{l}99 \pm 2 \\
67 \pm 8\end{array}$ & $\begin{array}{c}121 \pm 5 \\
136 \pm 60\end{array}$ & $\begin{array}{l}54 \pm 2 \\
74 \pm 9\end{array}$ & $\begin{array}{l}132 \pm 4 \\
96 \pm 21\end{array}$ & $\begin{array}{l}1.33 \pi \\
1.15 \pi\end{array}$ & $\begin{array}{c}5.87 \pm 0.16 \\
20.6 \pm 4.9\end{array}$ & $\begin{array}{c}24.4 \pm 1.4 \\
74.4 \pm 40.3\end{array}$ & $\begin{array}{c}1.5 \pm 2.0 \\
-3.5 \pm 38.6\end{array}$ & $\begin{array}{c}25.9 \pm 2.5 \\
70.9 \pm 55.8\end{array}$ \\
\hline $\begin{array}{l}08.66 \text { Sept. } 2015 \\
\mathrm{H}_{2}^{18} \mathrm{O} \\
08.84 \text { Sept. } 2015\end{array}$ & $107 \pm 2$ & $142 \pm 5$ & $132 \pm 2$ & $116 \pm 2$ & $1.35 \pi$ & $3.18 \pm 0.06$ & $13.5 \pm 0.6$ & $0.1 \pm 0.2$ & $13.6 \pm 0.6$ \\
\hline $\begin{array}{l}\mathrm{H}_{2}^{18} \mathrm{O} \\
08.73 \text { Sept. } 2015\end{array}$ & $114 \pm 2$ & $150 \pm 11$ & $131 \pm 2$ & $122 \pm 3$ & $1.46 \pi$ & $3.14 \pm 0.08$ & $14.4 \pm 1.0$ & $0.2 \pm 0.2$ & $14.6 \pm 1.0$ \\
\hline $\mathrm{H}_{2}^{17} \mathrm{O}$ & $105 \pm 6$ & $158 \pm 23$ & $(132)$ & $(115 \pm 10)$ & $1.44 \pi$ & $0.54 \pm 0.05$ & $2.43 \pm 0.44$ & $-0.30 \pm 0.21$ & $2.13 \pm 0.49$ \\
\hline $\mathrm{CH}_{3} \mathrm{OH}(3-2)$ & $114 \pm 7$ & $144 \pm 29$ & $133 \pm 3$ & $114 \pm 7$ & $1.35 \pi$ & $3 \pm 2.0$ & $132.5 \pm 26.1$ & $-1.9 \pm 8.4$ & $130.6 \pm 27.4$ \\
\hline $\mathrm{CH}_{3} \mathrm{OH}(12-11)$ & $127 \pm 20$ & $90 \pm 24$ & $128 \pm 5$ & $87 \pm 15$ & $0.8 \pi$ & $35.8 \pm 5.9$ & $85.5 \pm 34.5$ & $-1.9 \pm 18.3$ & $83.6 \pm 39.1$ \\
\hline $\mathrm{NH}_{3}$ & $106 \pm 3$ & $151 \pm 13$ & $129 \pm 3$ & $128 \pm 4$ & $1.51 \pi$ & $5.70 \pm 0.19$ & $27.1 \pm 2.4$ & $-0.5 \pm 0.7$ & $26.6 \pm 2.5$ \\
\hline $\mathrm{CO}$ & $130 \pm 90$ & $150 \pm 70$ & $122 \pm 31$ & $73 \pm 40$ & $1.0 \pi$ & $20.0 \pm 7.3$ & $62.8 \pm 39.2$ & $19.7 \pm 26.2$ & $82.5 \pm 47.1$ \\
\hline $\begin{array}{l}14.20 \text { Sept. } 2015 \\
\mathrm{H}_{2}^{18} \mathrm{O} \\
14.37 \text { Sept. } 2015\end{array}$ & $243 \pm 1$ & $127 \pm 2$ & $112 \pm 0$ & $105 \pm 1$ & $1.15 \pi$ & $3.42 \pm 0.03$ & $12.4 \pm 0.2$ & $0.8 \pm 0.1$ & $13.2 \pm 0.3$ \\
\hline $\begin{array}{l}\mathrm{H}_{2}^{18} \mathrm{O} \\
14.61 \text { Sept. } 2015\end{array}$ & $255 \pm 1$ & $124 \pm 2$ & $113 \pm 0$ & $95 \pm 1$ & $1.04 \pi$ & $3.49 \pm 0.05$ & $11.4 \pm 0.3$ & $1.0 \pm 0.2$ & $12.4 \pm 0.3$ \\
\hline $\begin{array}{l}\mathrm{H}_{2}^{18} \mathrm{O} \\
14.40 \text { Sept. } 2015\end{array}$ & $247 \pm 1$ & $126 \pm 2$ & $114 \pm 0$ & $96 \pm 1$ & $1.07 \pi$ & $3.42 \pm 0.04$ & $11.5 \pm 0.3$ & $1.1 \pm 0.2$ & $12.6 \pm 0.3$ \\
\hline $\mathrm{H}_{2}^{17} \mathrm{O}$ & $240 \pm 4$ & $145 \pm 15$ & $(114)$ & $(100)$ & $1.23 \pi$ & $0.48 \pm 0.02$ & $1.84 \pm 0.29$ & $0.06 \pm 0.13$ & $1.90 \pm 0.32$ \\
\hline $\mathrm{CH}_{3} \mathrm{OH}(3-2)$ & $238 \pm 2$ & $109 \pm 6$ & $113 \pm 1$ & $95 \pm 2$ & $0.94 \pi$ & $32.43 \pm 0.78$ & $96.0 \pm 5.9$ & $15.3 \pm$ & $111.3 \pm 7.4$ \\
\hline $\mathrm{CH}_{3} \mathrm{OH}(8-7)$ & $240 \pm 10$ & $102 \pm 16$ & $114 \pm 3$ & $90 \pm 7$ & $0.86 \pi$ & $29.36 \pm 2.50$ & $79.0 \pm 16.0$ & $10.4 \pm$ & $89.4 \pm 28.6$ \\
\hline $\mathrm{CH}_{3} \mathrm{OH}(12-11)$ & $243 \pm 3$ & $84 \pm 7$ & $118 \pm 1$ & $92 \pm 3$ & $0.74 \pi$ & \pm 1.98 & $98.1 \pm 9.9$ & $7.9 \pm$ & $106.0 \pm 22.4$ \\
\hline $\mathrm{NH}_{3}$ & $261 \pm 1$ & $122 \pm 3$ & $123 \pm 1$ & $116 \pm 2$ & $1.21 \pi$ & \pm 0.08 & $20.0 \pm 0.6$ & $1.5 \pm$ & $21.4 \pm 0.9$ \\
\hline $\mathrm{CO}$ & $265 \pm 13$ & $76 \pm 28$ & $102 \pm 13$ & $67 \pm 25$ & $0.51 \pi$ & $15.3 \pm 3.7$ & $26.0 \pm 17.1$ & & $24.4 \pm 30.4$ \\
\hline $\begin{array}{l}02.00 \text { Oct. } 2015 \\
\mathrm{H}_{2}^{18} \mathrm{O} \\
02.23 \text { Oct. } 2015\end{array}$ & $224 \pm 2$ & $115 \pm 15$ & $41 \pm 1$ & $119 \pm 1$ & $1.17 \pi$ & $2.65 \pm 0.09$ & $9.77 \pm 1.10$ & $1.1 \pm 0.4$ & $10.9 \pm 1.2$ \\
\hline $\begin{array}{l}\mathrm{H}_{2}^{18} \mathrm{O} \\
02.44 \text { Oct. } 2015\end{array}$ & $221 \pm 2$ & $139 \pm 6$ & $46 \pm 1$ & $113 \pm 1$ & $1.31 \pi$ & $2.60 \pm 0.06$ & $10.7 \pm 0.5$ & $0.1 \pm 0.2$ & $10.8 \pm 0.5$ \\
\hline $\begin{array}{l}\mathrm{H}_{2}^{18} \mathrm{O} \\
02.67 \text { Oct. } 2015\end{array}$ & $225 \pm 2$ & $150 \pm 30$ & $42 \pm 1$ & $101 \pm 1$ & $1.27 \pi$ & $2.59 \pm 0.07$ & $10.3 \pm 1.6$ & $0.7 \pm 0.3$ & $11.1 \pm 1.7$ \\
\hline $\begin{array}{l}\mathrm{H}_{2}^{18} \mathrm{O} \\
02.33 \text { Oct. } 2015\end{array}$ & $230 \pm 2$ & $145 \pm 12$ & $54 \pm 1$ & $117 \pm 1$ & $1.38 \pi$ & $2.32 \pm 0.06$ & $10.1 \pm 0.7$ & $0.9 \pm 0.3$ & $10.9 \pm 0.8$ \\
\hline $\mathrm{H}_{2}^{17} \mathrm{O}$ & $220 \pm 6$ & $150 \pm 15$ & $(46)$ & $(115)$ & $1.40 \pi$ & $0.510 \pm 0.042$ & $2.24 \pm 0.36$ & $-0.08 \pm 0.35$ & $2.16 \pm 0.50$ \\
\hline $\mathrm{CH}_{3} \mathrm{OH}(3-2)$ & $209 \pm 4$ & $0 \pm 12$ & $33 \pm 2$ & $3 \pm 3$ & $17 \pi$ & 0.68 & $67.4 \pm 7.3$ & $0.0 \pm$ & $67.4 \pm$ \\
\hline $\mathrm{CH}_{3} \mathrm{OH}$ & $223 \pm 9$ & $107 \pm 40$ & $62 \pm 8$ & $5 \pm 17$ & $1.08 \pi$ & $=4.90$ & $100.0 \pm 43.2$ & $50.0 \pm 46.5$ & $150.0 \pm 63.5$ \\
\hline $\mathrm{CH}_{3} \mathrm{OH}($ & $243 \pm 13$ & $127 \pm 40$ & $47 \pm 3$ & $100 \pm 10$ & $1.12 \pi$ & 3.73 & $105.3 \pm 35.5$ & $11.7 \pm$ & $117.0 \pm$ \\
\hline $\mathrm{NH}_{3}$ & $241 \pm 2$ & $112 \pm 5$ & $54 \pm 2$ & $109 \pm 4$ & $1.08 \pi$ & $3.53 \pm 0.10$ & $11.93 \pm 0.82$ & $2.27 \pm 1.29$ & $14.10 \pm 1.53$ \\
\hline $\mathrm{CO}$ & $244 \pm 32$ & $123 \pm 62$ & $37 \pm 11$ & $73 \pm 13$ & $0.87 \pi$ & $18.8 \pm 5.0$ & $51.4 \pm 30.4$ & $-1.6 \pm 8.6$ & $45.0 \pm 45.7$ \\
\hline $\begin{array}{l}17.34 \text { Oct. } 2015 \\
\mathrm{H}_{2}^{18} \mathrm{O} \\
17.52 \text { Oct. } 2015\end{array}$ & $257 \pm 1$ & $119 \pm 2$ & $98 \pm 1$ & $111 \pm 1$ & $1.15 \pi$ & $1.99 \pm 0.05$ & $7.15 \pm 0.25$ & $0.78 \pm 0.19$ & $7.93 \pm 0.31$ \\
\hline $\begin{array}{l}\mathrm{H}_{2}^{18} \mathrm{O} \\
17.71 \text { Oct. } 2015\end{array}$ & $252 \pm 1$ & $136 \pm 4$ & $96 \pm 1$ & $104 \pm 1$ & $1.21 \pi$ & $1.96 \pm 0.04$ & $7.41 \pm 0.27$ & $0.83 \pm 0.21$ & $8.24 \pm 0.34$ \\
\hline $\begin{array}{l}\mathrm{H}_{2}^{18} \mathrm{O} \\
17.43 \text { Oct. } 2015\end{array}$ & $254 \pm 1$ & $128 \pm 2$ & $103 \pm 1$ & $107 \pm 1$ & $1.18 \pi$ & $1.97 \pm 0.03$ & $7.29 \pm 0.19$ & $0.40 \pm 0.16$ & $7.69 \pm 0.25$ \\
\hline $\mathrm{H}_{2}^{17} \mathrm{O}$ & $256 \pm 3$ & $115 \pm 7$ & $(100)$ & $(115)$ & $1.15 \pi$ & $0.352 \pm 0.017$ & $1.27 \pm 0.14$ & $0.14 \pm 0.12$ & $1.41 \pm 0.18$ \\
\hline $\mathrm{CH}_{3} \mathrm{OH}(3-2)$ & $41 \pm 3$ & $128 \pm 8$ & $104 \pm 1$ & $89 \pm 3$ & $1.02 \pi$ & $17.85 \pm 0.65$ & $57.3 \pm 4.6$ & $2.5 \pm 5.4$ & $59.8 \pm 7.1$ \\
\hline $\mathrm{CH}_{3} \mathrm{OH}(8-7)$ & $259 \pm 14$ & $123 \pm 30$ & & $108 \pm 11$ & $1.15 \pi$ & $18.56 \pm 2.52$ & $67.1 \pm 19.7$ & $-4.0 \pm 22.0$ & $63.1 \pm 29.5$ \\
\hline $\mathrm{CH}_{3} \mathrm{OH}(12-11)$ & $246 \pm 9$ & $107 \pm 22$ & $99 \pm 2$ & $82 \pm 8$ & $0.84 \pi$ & $22.70 \pm 2.99$ & $59.9 \pm 16.3$ & $21.4 \pm 20.1$ & $81.3 \pm 25.9$ \\
\hline $\mathrm{NH}_{3}$ & $267 \pm 2$ & $126 \pm 4$ & $95 \pm 2$ & $96 \pm 5$ & $1.07 \pi$ & $2.05 \pm 0.06$ & $6.87 \pm 0.49$ & $1.11 \pm 0.88$ & $7.98 \pm 1.01$ \\
\hline $\begin{array}{l}24.33 \text { Oct. } 2015 \\
\mathrm{H}_{2}^{18} \mathrm{O} \\
24.51 \text { Oct. } 2015\end{array}$ & $118 \pm 1$ & $137 \pm 3$ & $89 \pm 1$ & $89 \pm 1$ & $1.08 \pi$ & $1.79 \pm 0.03$ & $6.06 \pm 0.17$ & $0.37 \pm 0.13$ & $6.43 \pm 0.21$ \\
\hline $\mathrm{H}_{2}^{18} \mathrm{O}$ & $124 \pm 2$ & $136 \pm 4$ & $88 \pm 1$ & $99 \pm 1$ & $1.17 \pi$ & $1.56 \pm 0.03$ & $5.70 \pm 0.22$ & $1.06 \pm 0.15$ & $6.76 \pm 0.27$ \\
\hline
\end{tabular}


Table A.3. continued.

\begin{tabular}{|c|c|c|c|c|c|c|c|c|c|}
\hline \multirow[t]{2}{*}{$\operatorname{Line}^{(b)}$} & \multicolumn{2}{|c|}{ Jet sky projection } & \multicolumn{2}{|c|}{ Jet Rosetta-jet plane } & \multirow{2}{*}{$\begin{array}{c}\Omega_{\mathrm{jet}}(d) \\
(\mathrm{sr}) \\
\end{array}$} & \multirow{2}{*}{$\begin{array}{c}q_{\mathrm{j}}\left[\times 10^{24}\right] \\
\left(\text { molec. } \mathrm{s}^{-1} \mathrm{sr}^{-1}\right)\end{array}$} & \multirow[t]{2}{*}{$Q_{\mathrm{J}}$} & \multirow{2}{*}{$\begin{array}{c}Q_{\mathrm{I}} \\
\left(\times 10^{24} \text { molec. } \mathrm{s}^{-1}\right)\end{array}$} & \multirow[t]{2}{*}{$Q_{\text {tot }}$} \\
\hline & $\mathrm{PA}\left({ }^{\circ}\right)$ & $\theta_{\mathrm{az}}{ }^{(c)}\left(^{\circ}\right)$ & Phase $\phi\left(^{\circ}\right)$ & $\theta_{\text {trans }}(c)\left({ }^{\circ}\right)$ & & & & & \\
\hline $\begin{array}{l}24.71 \text { Oct. } 2015 \\
\mathrm{H}_{2}^{18} \mathrm{O} \\
24.94 \text { Oct. } 2015\end{array}$ & $122 \pm 1$ & $131 \pm 3$ & $81 \pm 1$ & $102 \pm 1$ & $1.16 \pi$ & $1.72 \pm 0.03$ & $6.26 \pm 0.21$ & $0.47 \pm 0.12$ & $6.73 \pm 0.24$ \\
\hline $\begin{array}{l}\mathrm{H}_{2}^{18} \mathrm{O} \\
24.54 \text { Oct. } 2015\end{array}$ & $119 \pm 1$ & $121 \pm 3$ & $78 \pm 1$ & $105 \pm 1$ & $1.11 \pi$ & $1.81 \pm 0.03$ & $6.32 \pm 0.18$ & $0.43 \pm 0.20$ & $6.75 \pm 0.27$ \\
\hline $\mathrm{H}_{2}^{17} \mathrm{O}$ & $124 \pm 6$ & $133 \pm 17$ & $(90)$ & $(100)$ & $1.15 \pi$ & $0.21 \pm 0.02$ & $0.77 \pm 0.15$ & $0.24 \pm 0.12$ & $1.01 \pm 0.19$ \\
\hline $\mathrm{CH}_{3} \mathrm{OH}(3-2)$ & $132 \pm 3$ & $120 \pm 7$ & $97 \pm 1$ & $90 \pm 3$ & $0.98 \pi$ & $15.51 \pm 0.51$ & $47.7 \pm 3.7$ & $-0.1 \pm 5.7$ & $47.6 \pm 6.8$ \\
\hline $\mathrm{CH}_{3} \mathrm{OH}(8-7)$ & $117 \pm 9$ & $125 \pm 36$ & $111 \pm 6$ & $101 \pm 16$ & $1.11 \pi$ & $14.23 \pm 2.67$ & $49.6 \pm 19.2$ & $13.4 \pm 29.6$ & $63.0 \pm 35.3$ \\
\hline $\mathrm{CH}_{3} \mathrm{OH}(12-11)$ & $118 \pm 4$ & $77 \pm 7$ & $104 \pm 3$ & $102 \pm 7$ & $0.75 \pi$ & $25.90 \pm 1.92$ & $61.2 \pm 9.6$ & $16.8 \pm 10.2$ & $78.0 \pm 14.0$ \\
\hline $\mathrm{NH}_{3}$ & $117 \pm 2$ & $104 \pm 4$ & $72 \pm 2$ & $112 \pm 4$ & $1.04 \pi$ & $1.70 \pm 0.06$ & $5.54 \pm 0.39$ & $1.32 \pm 0.77$ & $6.86 \pm 0.06$ \\
\hline $\mathrm{CO}$ & $106 \pm 10$ & $80 \pm 19$ & $113 \pm 16$ & $92 \pm 31$ & $0.72 \pi$ & $9.5 \pm 3.1$ & $21.5 \pm 12.5$ & $-7.5 \pm 34.4$ & $14.0 \pm 36.6$ \\
\hline $\begin{array}{l}\text { 11.34 Nov. } 2015 \\
\mathrm{H}_{2}^{18} \mathrm{O} \\
11.53 \text { Nov. } 2015\end{array}$ & $259 \pm 1$ & $114 \pm 2$ & $88 \pm 1$ & $104 \pm 3$ & $1.05 \pi$ & $1.06 \pm 0.03$ & $3.51 \pm 0.15$ & $1.04 \pm 0.18$ & $4.55 \pm 0.23$ \\
\hline $\begin{array}{l}\mathrm{H}_{2}^{18} \mathrm{O} \\
11.73 \text { Nov. } 2015\end{array}$ & $262 \pm 1$ & $123 \pm 2$ & $88 \pm 1$ & $106 \pm 2$ & $1.13 \pi$ & $1.10 \pm 0.02$ & $3.92 \pm 0.13$ & $0.43 \pm 0.15$ & $4.35 \pm 0.20$ \\
\hline $\begin{array}{l}\mathrm{H}_{2}^{18} \mathrm{O} \\
11.56 \text { Nov. } 2015\end{array}$ & $270 \pm 3$ & $175 \pm 13$ & $95 \pm 3$ & $87 \pm 1$ & $1.27 \pi$ & $1.05 \pm 0.03$ & $4.21 \pm 0.28$ & $0.18 \pm 0.15$ & $4.39 \pm 0.32$ \\
\hline $\mathrm{H}_{2}^{17} \mathrm{O}$ & $258 \pm 5$ & $155 \pm 48$ & $(90)$ & $(90)$ & $1.20 \pi$ & $0.20 \pm 0.01$ & $0.74 \pm 0.15$ & $-0.21 \pm 0.08$ & $0.53 \pm 0.29$ \\
\hline $\mathrm{CH}_{3} \mathrm{OH}(3-2)$ & $253 \pm 3$ & $116 \pm 7$ & $112 \pm 2$ & $92 \pm 3$ & $0.97 \pi$ & $11.24 \pm$ & $34.2 \pm$ & $9.4 \pm$ & $43.6 \pm$ \\
\hline $\mathrm{CH}_{3} \mathrm{OH}(8-7)$ & $257 \pm 12$ & $79 \pm 15$ & $101 \pm 6$ & $87 \pm 20$ & $0.68 \pi$ & $9.32 \pm 2.24$ & $19.9 \pm 8.5$ & $24.9 \pm 17.4$ & $43.8 \pm 19.6$ \\
\hline $\mathrm{CH}_{3} \mathrm{OH}(12-11)$ & $261 \pm 10$ & $130 \pm 26$ & $108 \pm 2$ & $98 \pm 5$ & $1.12 \pi$ & $15.53 \pm$ & $54.4 \pm 11.6$ & $-4.4 \pm$ & $50.0 \pm 17.5$ \\
\hline $\mathrm{NH}_{3}$ & $278 \pm 2$ & $3 \pm 5$ & $95 \pm 2$ & $94 \pm 8$ & $1.10 \pi$ & $7 \pm 0.04$ & $71 \pm 0.38$ & $0.01 \pm$ & $3.72 \pm 0.75$ \\
\hline $\mathrm{CO}$ & $251 \pm 47$ & $105 \pm 47$ & $83 \pm 14$ & $42 \pm 24$ & $0.51 \pi$ & $8.4 \pm 2.4$ & $13.4 \pm 11.2$ & $3.3 \pm$ & $16.7 \pm 21.2$ \\
\hline $\begin{array}{l}21.95 \text { Nov. } 2015 \\
\mathrm{H}_{2}^{18} \mathrm{O} \\
22.13 \text { Nov. } 2015\end{array}$ & $280 \pm 2$ & $108 \pm 5$ & $43 \pm 1$ & $132 \pm 1$ & $1.22 \pi$ & $0.67 \pm 0.01$ & $2.56 \pm 0.11$ & $0.33 \pm 0.15$ & $2.89 \pm 0.19$ \\
\hline $\begin{array}{l}\mathrm{H}_{2}^{18} \mathrm{O} \\
22.36 \text { Nov. } 2015\end{array}$ & $287 \pm 3$ & $130 \pm 7$ & $62 \pm 1$ & $127 \pm 1$ & $1.36 \pi$ & $0.76 \pm 0.02$ & $3.24 \pm 0.17$ & $0.25 \pm 0.14$ & $3.49 \pm 0.22$ \\
\hline $\begin{array}{l}\mathrm{H}_{2}^{18} \mathrm{O} \\
22.15 \text { Nov. } 2015\end{array}$ & $273 \pm 2$ & $116 \pm 7$ & $42 \pm 1$ & $117 \pm 1$ & $1.17 \pi$ & $0.70 \pm 0.02$ & $2.55 \pm 0.12$ & $0.33 \pm 0.11$ & $2.88 \pm 0.16$ \\
\hline $\mathrm{H}_{2}^{17} \mathrm{O}$ & $277 \pm 11$ & $85 \pm 25$ & $(45)$ & $(125)$ & $0.97 \pi$ & $0.08 \pm 0.02$ & $0.24 \pm 0.10$ & $0.38 \pm 0.15$ & $0.62 \pm 0.18$ \\
\hline $\mathrm{CH}_{3} \mathrm{OH}(3-2)$ & $4 \pm 12$ & $144 \pm 35$ & $27 \pm 2$ & $127 \pm 4$ & $1.45 \pi$ & $5.76 \pm$ & $26.2 \pm$ & $5.4=$ & $31.6=$ \\
\hline $\mathrm{CH}_{3} \mathrm{OH}(8-7)$ & $6 \pm 11$ & $136 \pm 74$ & $42 \pm 10$ & $163 \pm 16$ & $1.65 \pi$ & $7.45 \pm$ & $38.6 \pm$ & $-3.5 \pm$ & $35.1 \pm$ \\
\hline $\mathrm{CH}_{3} \mathrm{OH}(12-11)$ & $2 \pm 7$ & $166 \pm 63$ & $29 \pm 2$ & $3 \pm 8$ & $1.70 \pi$ & $10.14 \pm 0.65$ & $4.2 \pm 15.0$ & -1 & $37.0 \pm$ \\
\hline $\mathrm{NH}_{3}$ & $56 \pm 2$ & $106 \pm 6$ & $67 \pm 4$ & $122 \pm 7$ & $1.12 \pi$ & $0.65 \pm 0.03$ & $2.30 \pm 0.23$ & $0.35 \pm$ & $2.65 \pm 0.54$ \\
\hline $\mathrm{CO}$ & $180 \pm 47$ & $120 \pm 50$ & $(45)$ & $(95)$ & $(1.0 \pi)$ & $2.0 \pm 1.0$ & $6.3 \pm 3.6$ & $7.3 \pm 8.4$ & $13.6 \pm 9.1$ \\
\hline $\begin{array}{l}03.16 \text { Dec. } 2015 \\
\mathrm{H}_{2}^{18} \mathrm{O} \\
03.38 \text { Dec. } 2015\end{array}$ & $89 \pm 2$ & $140 \pm 8$ & $122 \pm 1$ & $130 \pm 2$ & $1.46 \pi$ & $0.48 \pm 0.01$ & $2.21 \pm 0.10$ & $0.01 \pm 0.13$ & $2.22 \pm 0.16$ \\
\hline $\begin{array}{l}\mathrm{H}_{2}^{18} \mathrm{O} \\
03.58 \text { Dec. } 2015\end{array}$ & $104 \pm 3$ & $145 \pm 16$ & $125 \pm 2$ & $122 \pm 2$ & $1.42 \pi$ & $0.48 \pm 0.02$ & $2.15 \pm 0.19$ & $0.09 \pm 0.11$ & $2.24 \pm 0.22$ \\
\hline $\begin{array}{l}\mathrm{H}_{2}^{18} \mathrm{O} \\
03.80 \text { Dec. } 2015\end{array}$ & $93 \pm 2$ & $150 \pm 13$ & $125 \pm 1$ & $116 \pm 3$ & $1.41 \pi$ & $0.44 \pm 0.01$ & $1.94 \pm 0.15$ & $0.04 \pm 0.09$ & $1.98 \pm 0.17$ \\
\hline $\begin{array}{l}\mathrm{H}_{2}^{18} \mathrm{O} \\
03.48 \text { Dec. } 2015\end{array}$ & $95 \pm 2$ & $127 \pm 5$ & $111 \pm 1$ & $116 \pm 3$ & $1.24 \pi$ & $0.44 \pm 0.01$ & $1.72 \pm 0.09$ & $0.26 \pm 0.10$ & $1.98 \pm 0.13$ \\
\hline $\mathrm{H}_{2}^{17} \mathrm{O}$ & $81 \pm 8$ & $146 \pm 39$ & (120) & $(120)$ & $1.41 \pi$ & $0.099 \pm 0.009$ & $0.44 \pm 0.11$ & $-0.02 \pm 0.15$ & $0.42 \pm 0.19$ \\
\hline $\mathrm{CH}_{3} \mathrm{OH}(3-2)$ & $86 \pm 9$ & $110 \pm 40$ & $155 \pm 3$ & $176 \pm 5$ & $1.50 \pi$ & $5.39 \pm 0.39$ & $25.4 \pm 7.0$ & $-0.4 \pm 4.4$ & $25.0 \pm 8.3$ \\
\hline $\mathrm{CH}_{3} \mathrm{OH}(12-11)$ & $117 \pm 9$ & $105 \pm 46$ & $118 \pm 4$ & $120 \pm 10$ & $1.11 \pi$ & $5.86 \pm 0.97$ & $20.4 \pm 8.8$ & $9.1 \pm 11.7$ & $29.5 \pm 14.6$ \\
\hline $\mathrm{NH}_{3}$ & $103 \pm 6$ & $117 \pm 16$ & $108 \pm 9$ & $147 \pm 16$ & $1.39 \pi$ & $0.38 \pm 0.04$ & $1.66 \pm 0.33$ & $0.31 \pm 0.56$ & $1.98 \pm 0.65$ \\
\hline $\begin{array}{l}28.20 \text { Dec. } 2015 \\
\mathrm{H}_{2}^{18} \mathrm{O} \\
28.41 \text { Dec. } 2015\end{array}$ & $124 \pm 3$ & $109 \pm 8$ & $103 \pm 1$ & $109 \pm 2$ & $1.06 \pi$ & $0.31 \pm 0.01$ & $1.02 \pm 0.07$ & $0.20 \pm 0.10$ & $1.22 \pm 0.12$ \\
\hline $\begin{array}{l}\mathrm{H}_{2}^{18} \mathrm{O} \\
28.61 \text { Dec. } 2015\end{array}$ & $121 \pm 3$ & $114 \pm 6$ & $101 \pm 1$ & $121 \pm 2$ & $1.18 \pi$ & $0.32 \pm 0.01$ & $1.17 \pm 0.07$ & $0.10 \pm 0.11$ & $1.28 \pm 0.13$ \\
\hline $\mathrm{H}_{2}^{18} \mathrm{O}$ & $146 \pm 2$ & $140 \pm 7$ & $98 \pm 1$ & $116 \pm 2$ & $1.34 \pi$ & $0.35 \pm 0.01$ & $1.48 \pm 0.06$ & $0.08 \pm 0.07$ & $1.56 \pm 0.10$ \\
\hline
\end{tabular}


Table A.3. continued.

\begin{tabular}{|c|c|c|c|c|c|c|c|c|c|}
\hline \multirow[t]{2}{*}{$\operatorname{Line}^{(b)}$} & \multicolumn{2}{|c|}{ Jet sky projection } & \multicolumn{2}{|c|}{ Jet Rosetta-jet plane } & \multirow{2}{*}{$\begin{array}{c}\Omega_{\mathrm{jet}}(d) \\
(\mathrm{sr}) \\
\end{array}$} & \multirow{2}{*}{$\begin{array}{c}q_{\mathrm{j}}\left[\times 10^{24}\right] \\
\left(\text { molec. } \mathrm{s}^{-1} \mathrm{sr}^{-1}\right)\end{array}$} & \multirow[t]{2}{*}{$Q_{\mathrm{J}}$} & \multirow{2}{*}{$\begin{array}{c}Q_{\mathrm{I}} \\
\left.\times 10^{24} \text { molec. }^{-1}\right) \\
\end{array}$} & \multirow[t]{2}{*}{$Q_{\text {tot }}$} \\
\hline & $\mathrm{PA}\left({ }^{\circ}\right)$ & $\theta_{\mathrm{az}}^{(c)}\left(^{\circ}\right)$ & Phase $\phi\left(^{\circ}\right)$ & $\theta_{\text {trans }}{ }^{(c)}\left({ }^{\circ}\right)$ & & & & & \\
\hline \multicolumn{10}{|l|}{28.40 Dec. 2015} \\
\hline $\mathrm{H}_{2}^{17} \mathrm{O}$ & $127 \pm 15$ & $108 \pm 22$ & $(100)$ & $(115)$ & $1.09 \pi$ & $0.06 \pm 0.01$ & $0.22 \pm 0.08$ & $0.16 \pm 0.14$ & $0.38 \pm 0.16$ \\
\hline $\mathrm{CH}_{3} \mathrm{OH}(3-2)$ & $165 \pm 9$ & $144 \pm 28$ & $95 \pm 3$ & $109 \pm 9$ & $1.30 \pi$ & $3.12 \pm 0.32$ & $12.8 \pm 2.7$ & $2.7 \pm 3.5$ & $15.4 \pm 4.4$ \\
\hline $\mathrm{CH}_{3} \mathrm{OH}(12-11)$ & $131 \pm 35$ & $140 \pm 40$ & $117 \pm 5$ & $102 \pm 12$ & $1.21 \pi$ & $4.17 \pm 0.69$ & $15.9 \pm 4.9$ & $-0.4 \pm 11.1$ & $15.5 \pm 12.1$ \\
\hline $\mathrm{NH}_{3}$ & $119 \pm 4$ & $70 \pm 8$ & $103 \pm 7$ & $85 \pm 17$ & $0.60 \pi$ & $0.30 \pm 0.04$ & $0.56 \pm 0.17$ & $0.06 \pm 0.36$ & $0.62 \pm 0.40$ \\
\hline \multicolumn{10}{|l|}{ 25.20 Jan. 2016} \\
\hline $\begin{array}{l}\mathrm{H}_{2}^{18} \mathrm{O} \\
25.38 \text { Jan. } 2016\end{array}$ & $218 \pm 7$ & $110 \pm 21$ & $31 \pm 2$ & $133 \pm 5$ & $1.24 \pi$ & $0.17 \pm 0.01$ & $0.65 \pm 0.11$ & $-0.04 \pm 0.12$ & $0.61 \pm 0.16$ \\
\hline $\begin{array}{l}\mathrm{H}_{2}^{18} \mathrm{O} \\
\text { 25.61 Jan. } 2016\end{array}$ & $247 \pm 10$ & $127 \pm 61$ & $23 \pm 2$ & $122 \pm 4$ & $1.25 \pi$ & $0.13 \pm 0.01$ & $0.53 \pm 0.21$ & $0.10 \pm 0.11$ & $0.63 \pm 0.24$ \\
\hline $\begin{array}{l}\mathrm{H}_{2}^{18} \mathrm{O} \\
25.41 \text { Jan. } 2016\end{array}$ & $251 \pm 6$ & $160 \pm 20$ & $53 \pm 3$ & $122 \pm 4$ & $1.52 \pi$ & $0.15 \pm 0.01$ & $0.73 \pm 0.09$ & $0.10 \pm 0.08$ & $0.83 \pm 0.12$ \\
\hline $\mathrm{CH}_{3} \mathrm{OH}(3-2)$ & $178 \pm 7$ & $96 \pm 44$ & $15 \pm 11$ & $167 \pm 19$ & $1.32 \pi$ & $2.38 \pm 0.32$ & $9.9 \pm 4.2$ & $0.3 \pm 5.3$ & $10.1 \pm 6.7$ \\
\hline $\mathrm{CH}_{3} \mathrm{OH}(12-11)$ & $228 \pm 7$ & $71 \pm 13$ & $41 \pm 6$ & $72 \pm 12$ & $0.52 \pi$ & $5.11 \pm 1.15$ & $8.4 \pm 3.2$ & $4.9 \pm 7.2$ & $13.2 \pm 7.8$ \\
\hline $\mathrm{NH}_{3}$ & $146 \pm 12$ & $105 \pm 50$ & $(40)$ & $(105)$ & $1.02 \pi$ & $0.09 \pm 0.02$ & $0.28 \pm 0.20$ & $-0.06 \pm 0.29$ & $0.22 \pm 0.35$ \\
\hline \multicolumn{10}{|l|}{28.15 Feb. 2016} \\
\hline $\mathrm{H}_{2}^{18} \mathrm{O}$ & $115 \pm 5$ & $118 \pm 13$ & $84 \pm 2$ & $114 \pm 4$ & $1.16 \pi$ & $0.059 \pm 0.003$ & $0.22 \pm 0.03$ & $0.05 \pm 0.03$ & $0.27 \pm 0.04$ \\
\hline $\mathrm{CH}_{3} \mathrm{OH}(3-2)$ & $142 \pm 9$ & $146 \pm 30$ & $90 \pm 4$ & $111 \pm 9$ & $1.33 \pi$ & $2.11 \pm 0.20$ & $8.9 \pm 2.0$ & $0.5 \pm 2.6$ & $9.4 \pm 3.3$ \\
\hline $\mathrm{NH}_{3}$ & $137 \pm 23$ & $138 \pm 50$ & $(90)$ & $(110)$ & $(1.25 \pi)$ & $0.04 \pm 0.01$ & $0.16 \pm 0.06$ & $0.05 \pm 0.11$ & $0.21 \pm 0.12$ \\
\hline \multicolumn{10}{|l|}{14.72 Mar. 2016} \\
\hline $\mathrm{H}_{2}^{18} \mathrm{O}$ & $98 \pm 5$ & $86 \pm 12$ & $106 \pm 3$ & $125 \pm 5$ & $0.98 \pi$ & $0.043 \pm 0.003$ & $0.13 \pm 0.02$ & $0.04 \pm 0.04$ & $0.17 \pm 0.05$ \\
\hline $\mathrm{CH}_{3} \mathrm{OH}(3-2)$ & $133 \pm 9$ & $62 \pm 17$ & $101 \pm 8$ & $126 \pm 19$ & $0.76 \pi$ & $1.75 \pm 0.31$ & $4.2 \pm 1.7$ & $1.9 \pm 1.5$ & $6.1 \pm 2.2$ \\
\hline $\mathrm{NH}_{3}$ & & $(90)$ & & (125) & $(1.0 \pi)$ & $0.04 \pm 0.02$ & $0.13 \pm 0.07$ & $(0.0)$ & $0.13 \pm 0.07$ \\
\hline \multicolumn{10}{|l|}{22.65 Apr. 2016} \\
\hline $\mathrm{H}_{2}^{16} \mathrm{O}$ & $64 \pm 1$ & $145 \pm 1$ & $106 \pm 1$ & $131 \pm 1$ & $1.49 \pi$ & $3.8 \pm 1.2$ & $17.8 \pm 5.6$ & $4.5 \pm 0.7$ & $22.3 \pm 5.6$ \\
\hline $\mathrm{H}_{2}^{18} \mathrm{O}$ & $106 \pm 7$ & $115 \pm 17$ & $84 \pm 7$ & $100 \pm 13$ & $1.03 \pi$ & $0.015 \pm 0.002$ & $0.05 \pm 0.01$ & $0.00 \pm 0.03$ & $0.05 \pm 0.03$ \\
\hline $\mathrm{CH}_{3} \mathrm{OH}(3-2)$ & $161 \pm 14$ & $86 \pm 24$ & $162 \pm 20$ & $106 \pm 35$ & $0.85 \pi$ & $0.62 \pm 0.09$ & $1.66 \pm 0.88$ & $(0.0)$ & \\
\hline 2nd jet: & $161 \pm 14$ & $86 \pm 24$ & $28 \pm 13$ & $146 \pm 35$ & $1.10 \pi$ & $0.74 \pm 0.27$ & $2.57 \pm 1.38$ & $(0.0)$ & $4.2 \pm 1.6$ \\
\hline $\mathrm{NH}_{3}$ & $40 \pm 30$ & $115 \pm 41$ & $(90)$ & (115) & $1.15 \pi$ & $0.04 \pm 0.01$ & $0.14 \pm 0.09$ & $0.26 \pm 0.20$ & $0.40 \pm 0.22$ \\
\hline
\end{tabular}


Table A.4. Production rates $Q_{\mathrm{j}}$ for the jet alone based on derived or assumed jet width and angular peak production rate derived from line intensities along the jet $\left( \pm 30^{\circ}\right)$.

\begin{tabular}{|c|c|c|c|c|c|c|}
\hline Date & $\begin{array}{l}\text { Average } Q_{\mathrm{j}}\left[\mathrm{H}_{2}^{16} \mathrm{O}\right] \\
\left(\times 10^{27} \text { molec. } \mathrm{s}^{-1}\right)\end{array}$ & $Q_{\mathrm{j}}\left[\mathrm{H}_{2}^{18} \mathrm{O}\right]$ & $Q_{\mathrm{j}}\left[\mathrm{H}_{2}^{17} \mathrm{O}\right]$ & $\begin{array}{l}Q_{\mathrm{j}}\left[\mathrm{CH}_{3} \mathrm{OH}\right] \\
\left.0^{24} \text { molec. } \mathrm{s}^{-1}\right)\end{array}$ & $Q_{\mathrm{j}}\left[\mathrm{NH}_{3}\right]$ & $Q_{\mathrm{j}}[\mathrm{CO}]$ \\
\hline 2014/10/13.1 & $0.05 \pm 0.01$ & $0.09 \pm 0.02$ & - & $0.07 \pm 0.31$ & $0.13 \pm 0.07$ & - \\
\hline 2014/11/23.2 & $0.05 \pm 0.01$ & $0.09 \pm 0.02$ & - & & $<0.37$ & - \\
\hline $2014 / 12 / 13.8$ & $0.06 \pm 0.01$ & $0.12 \pm 0.02$ & - & $1.2 \pm 0.6$ & $0.12 \pm 0.09$ & - \\
\hline 2015/01/01.5 & $0.11 \pm 0.01$ & $0.21 \pm 0.01$ & - & $2.0 \pm 0.5$ & $0.22 \pm 0.12$ & - \\
\hline 2015/01/18.4 & $0.13 \pm 0.01$ & $0.26 \pm 0.03$ & - & $1.6 \pm 0.9$ & $0.50 \pm 0.16$ & - \\
\hline $2015 / 02 / 16.1$ & $0.15 \pm 0.01$ & $0.30 \pm 0.02$ & - & $1.9 \pm 0.5$ & $0.27 \pm 0.14$ & - \\
\hline 2015/03/14.8 & $0.39 \pm 0.08$ & $0.77 \pm 0.15$ & - & $4.7 \pm 2.2$ & $1.05 \pm 0.45$ & - \\
\hline $2015 / 03 / 22.4$ & $0.29 \pm 0.02$ & $0.59 \pm 0.04$ & - & $3.4 \pm 1.1$ & $1.56 \pm 0.51$ & - \\
\hline $2015 / 05 / 15.7$ & $0.65 \pm 0.06$ & $1.31 \pm 0.11$ & - & $8.9 \pm 3.0$ & $5.2 \pm 0.6$ & - \\
\hline 2015/06/07.1 & $1.6 \pm 0.2$ & $3.18 \pm 0.10$ & $0.57 \pm 0.12$ & $11.4 \pm 2.4$ & $9.0 \pm 0.4$ & $4.9 \pm 2.7$ \\
\hline 2015/06/14.2 & $1.8 \pm 0.1$ & $3.68 \pm 0.11$ & - & $22.2 \pm 5.1$ & $7.9 \pm 0.5$ & $28.1 \pm 14.2$ \\
\hline $2015 / 06 / 24.7$ & $2.3 \pm 0.1$ & $4.60 \pm 0.11$ & - & $24.7 \pm 4.3$ & $10.2 \pm 0.4$ & $<19.7$ \\
\hline 2015/07/13.2 & $3.2 \pm 0.1$ & $6.30 \pm 0.20$ & - & $37.2 \pm 6.6$ & $18.2 \pm 0.9$ & - \\
\hline 2015/07/23.4 & $5.0 \pm 0.1$ & $9.96 \pm 0.19$ & $1.88 \pm 0.16$ & $36.6 \pm 5.8$ & $22.6 \pm 1.0$ & $8.9 \pm 10.3$ \\
\hline $2015 / 07 / 30.6$ & $4.7 \pm 0.1$ & $9.29 \pm 0.21$ & $1.57 \pm 0.21$ & $54.5 \pm 6.1$ & $25.3 \pm 0.9$ & $26.3 \pm 20.9$ \\
\hline 2015/08/02.2 & $5.8 \pm 0.1$ & $11.65 \pm 0.22$ & $1.61 \pm 0.23$ & $48.8 \pm 4.4$ & $25.8 \pm 1.0$ & $19.9 \pm 9.5$ \\
\hline 2015/08/09.1 & $6.6 \pm 0.2$ & $13.20 \pm 0.41$ & $1.78 \pm 0.25$ & $75.7 \pm 9.8$ & $24.2 \pm 1.4$ & $25.4 \pm 8.1$ \\
\hline $2015 / 08 / 27.6$ & $7.2 \pm 0.1$ & $14.36 \pm 0.22$ & $2.19 \pm 0.35$ & $97.0 \pm 8.0$ & $24.5 \pm 0.8$ & $66.0 \pm 36.3$ \\
\hline 2015/09/01.7 & $6.4 \pm 0.1$ & $12.88 \pm 0.25$ & $2.29 \pm 0.32$ & $93.1 \pm 9.4$ & $25.2 \pm 1.4$ & $69.8 \pm 37.1$ \\
\hline 2015/09/08.7 & $5.5 \pm 0.2$ & $11.07 \pm 0.39$ & $2.11 \pm 0.35$ & $95.1 \pm 16.3$ & $22.5 \pm 1.9$ & $59.8 \pm 43.4$ \\
\hline 2015/09/14.4 & $5.6 \pm 0.1$ & $11.16 \pm 0.13$ & $1.91 \pm 0.29$ & $93.6 \pm 4.5$ & $19.5 \pm 0.6$ & $10.7 \pm 7.2$ \\
\hline 2015/10/02.4 & $4.8 \pm 0.2$ & $9.56 \pm 0.34$ & $2.01 \pm 0.28$ & $68.1 \pm 8.0$ & $12.4 \pm 0.8$ & $27.0 \pm 16.4$ \\
\hline 2015/10/17.5 & $3.9 \pm 0.1$ & $6.78 \pm 0.12$ & $1.28 \pm 0.13$ & $55.3 \pm 4.1$ & $8.0 \pm 0.5$ & $24.8 \pm 8.8$ \\
\hline $2015 / 10 / 24.6$ & $2.9 \pm 0.1$ & $5.70 \pm 0.08$ & $0.98 \pm 0.17$ & $47.2 \pm 3.3$ & $6.9 \pm 0.5$ & $25.4 \pm 14.4$ \\
\hline 2015/11/11.6 & $1.77 \pm 0.04$ & $3.54 \pm 0.07$ & $0.54 \pm 0.20$ & $34.6 \pm 2.8$ & $4.07 \pm 0.40$ & $9.5 \pm 8.3$ \\
\hline 2015/11/22.2 & $1.31 \pm 0.04$ & $2.62 \pm 0.07$ & $0.35 \pm 0.13$ & $32.2 \pm 7.4$ & $2.65 \pm 0.72$ & $4.9 \pm 3.1$ \\
\hline 2015/12/03.5 & $0.91 \pm 0.03$ & $1.82 \pm 0.05$ & $0.38 \pm 0.09$ & $21.5 \pm 4.9$ & $2.21 \pm 0.39$ & $5.4 \pm 2.8$ \\
\hline $2015 / 12 / 28.4$ & $0.61 \pm 0.02$ & $1.19 \pm 0.03$ & $0.23 \pm 0.07$ & $11.6 \pm 1.9$ & $0.54 \pm 0.15$ & $3.8 \pm 2.6$ \\
\hline $2016 / 01 / 25.4$ & $0.28 \pm 0.03$ & $0.56 \pm 0.05$ & $0.21 \pm 0.02$ & $8.7 \pm 2.4$ & $0.20 \pm 0.15$ & $5.4 \pm 2.8$ \\
\hline $2016 / 02 / 28.2$ & $0.12 \pm 0.02$ & $0.24 \pm 0.03$ & - & $7.9 \pm 1.9$ & $<0.18$ & - \\
\hline 2016/03/14.7 & $0.08 \pm 0.02$ & $0.15 \pm 0.02$ & - & $5.2 \pm 2.0$ & $0.08 \pm 0.06$ & - \\
\hline 2016/04/12.7 & $0.03 \pm 0.01$ & $0.08 \pm 0.02$ & - & $4.9 \pm 1.9$ & $0.28 \pm 0.15$ & - \\
\hline $2016 / 04 / 22.6$ & $0.02 \pm 0.01$ & $0.03 \pm 0.01$ & - & $5.3 \pm 2.6$ & $0.08 \pm 0.10$ & - \\
\hline
\end{tabular}


Table A.5. Line-of-sight abundances relative to water derived from nadir pointing.

\begin{tabular}{|c|c|c|c|c|c|c|c|}
\hline yyyy/mm/dd.dd-dd.dd & $(\mathrm{AU})$ & $\begin{array}{r}\text { Subsolar }{ }^{(a)} \\
\text { latitude } \\
\left({ }^{\circ}\right)\end{array}$ & $\begin{array}{c}\text { Sub S/C }{ }^{(b)} \\
\text { colatitude } \\
\phi\left({ }^{\circ}\right)\end{array}$ & (molec. $\mathrm{s}^{-1} \mathrm{st}^{-1}$ ) & $\mathrm{CH}_{3} \mathrm{OH}$ & Abundance in & $\mathrm{CO}$ \\
\hline 2014/08/19.1 -31.9 & 3.457 & +43.46 & $39 . \pm 45$ & $113 \pm 11 \times 10^{23}$ & $0.87 \pm 0.13$ & $0.13 \pm 0.02$ & $0.00 \pm 0.38$ \\
\hline $2014 / 09 / 21.52-23.02$ & 3.320 & +41.61 & $44.6 \pm 6.2$ & $149 \pm 3 \times 10^{23}$ & $1.23 \pm 0.14$ & $0.17 \pm 0.03$ & $1.90 \pm 0.47$ \\
\hline 2014/11/09.20-10.86 & 3.008 & +37.45 & & $211 \pm 3 \times 10^{23}$ & $0.64 \pm 0.19$ & $0.17 \pm 0.02$ & $0.88 \pm 0.46$ \\
\hline 2014/11/29.24-29.99 & 2.875 & +35.39 & $39.8 \pm 1.3$ & $187 \pm 8 \times 10^{23}$ & $0.51 \pm 0.24$ & $0.28 \pm 0.07$ & $0.00 \pm 0.93$ \\
\hline $2014 / 12 / 26.54-29.58$ & 2.675 & +31.86 & $49.0 \pm 9.5$ & $147 \pm 2 \times 10^{23}$ & $1.25 \pm 0.21$ & $0.32 \pm 0.05$ & $0.00 \pm 0.75$ \\
\hline $2015 / 02 / 16.55-17.00$ & 2.300 & +23.54 & $92.0 \pm 1.0$ & $116 \pm 2 \times 10^{23}$ & $0.65 \pm 0.42$ & $0.25 \pm 0.11$ & $0.00 \pm 1.94$ \\
\hline 2015/03/11.24-12.02 & 2.126 & +18.60 & $100.0 \pm 8.0$ & $304 \pm 62 \times 10^{23}$ & $0.72 \pm 0.15$ & $0.26 \pm 0.09$ & $0.00 \pm 1.34$ \\
\hline 2015/04/21.13-23.01 & 1.807 & +6.73 & $12.0 \pm 4.0$ & $914 \pm 49 \times 10^{23}$ & $0.71 \pm 0.09$ & $0.37 \pm 0.02$ & $0.00 \pm 0.143$ \\
\hline 2015/05/02.02-04.96 & 1.721 & +2.58 & $144.0 \pm 3.5$ & $717 \pm 63 \times 10^{23}$ & $1.07 \pm 0.11$ & $0.64 \pm 0.06$ & $0.19 \pm 0.12$ \\
\hline 2015/05/08.17-09.84 & 1.681 & +0.56 & $94.5 \pm 14$ & $1210 \pm 34 \times 10^{23}$ & $0.48 \pm 0.10$ & $0.26 \pm 0.08$ & $0.18 \pm 0.13$ \\
\hline 2015/05/11.01-12.02 & 1.661 & -0.43 & $56.8 \pm 6.6$ & $1035 \pm 59 \times 10^{23}$ & $0.38 \pm 0.03$ & $0.20 \pm 0.01$ & $0.00 \pm 0.133$ \\
\hline $2015 / 05 / 16.01-16.92$ & 1.627 & -2.46 & $31.8 \pm 3.7$ & $1139 \pm 106 \times 10^{23}$ & $0.64 \pm 0.08$ & $0.53 \pm 0.05$ & $0.24 \pm 0.187$ \\
\hline $2015 / 05 / 30.01-32.96$ & 1.522 & -9.38 & $167.0 \pm 7.5$ & $1373 \pm 80 \times 10^{23}$ & $1.00 \pm 0.06$ & $0.72 \pm 0.04$ & $0.00 \pm 0.117$ \\
\hline 2015/06/19.00-20.86 & 1.406 & -19.10 & $23.0 \pm 11$ & $2153 \pm 120 \times 10^{23}$ & $0.54 \pm 0.06$ & & $0.13 \pm 0.07$ \\
\hline 2015/07/10.01-11.01 & 1.310 & -30.70 & $85.8 \pm 4.2$ & $3449 \pm 186 \times 10^{23}$ & $0.77 \pm 0.08$ & $0.17 \pm 0.01$ & $0.12 \pm 0.047$ \\
\hline $2015 / 07 / 31.07-31.50$ & 1.253 & -42.16 & $135.4 \pm 1.3$ & $12288 \pm 736 \times 10^{23}$ & $1.69 \pm 0.18$ & $0.31 \pm 0.02$ & $0.18 \pm 0.02$ \\
\hline 2015/08/16.65 & 1.244 & -49.21 & $.1 \pm 1.5$ & $2105 \pm 360 \times 10^{23}$ & $1.04 \pm 0.20$ & $0.51 \pm 0.09$ & $0.09 \pm 0.138$ \\
\hline-21.15 & 1.247 & -50.30 & 1.7 & $4743 \pm 334 \times 10^{23}$ & $1.14 \pm 0.17$ & $0.28 \pm 0.02$ & $0.11 \pm 0.08$ \\
\hline $2015 / 08 / 23.01-23.51$ & 1.250 & -50.85 & $94.9 \pm 2.7$ & $6590 \pm 480 \times 10^{23}$ & $1.22 \pm 0.21$ & $0.32 \pm 0.02$ & $0.19 \pm 0.04$ \\
\hline /08/26.61-27.02 & 1.25 & -51.50 & $126.0 \pm 2.1$ & $10490 \pm 883 \times 10^{23}$ & $1.85 \pm 0.20$ & $0.30 \pm 0.03$ & $0.37 \pm 0.04$ \\
\hline & 1.2 & -51.97 & $147.1 \pm 0.5$ & $16031 \pm 602 \times 10^{23}$ & \pm 0.22 & $0.42 \pm$ & $0.48 \pm$ \\
\hline 2015/09/05.51-06.01 & 1.27 & -52.17 & & $5100 \pm 320 \times 10^{23}$ & $1.12 \pm 0.23$ & $0.63 \pm c$ & $0.12 \pm 0.05$ \\
\hline 2015/09/21.55-21.96 & 1.335 & -49.98 & $75.0 \pm 1.0$ & $5141 \pm 289 \times 10^{23}$ & $1.22 \pm 0.26$ & $0.40 \pm 0.02$ & $0.11 \pm 0.08$ \\
\hline & & -49.08 & & $8769 \pm 605 \times 10^{23}$ & $1.85 \pm 0.26$ & $0.31 \pm 0$ & $0.21 \pm 0.05$ \\
\hline & & -46.55 & \pm 0.1 & $8200 \pm 3100 \times 10^{23}$ & $=1.07$ & 0.1 & $1.03 \pm$ \\
\hline $2015 / 10 / 10.03-10.44$ & 1.428 & -44.28 & $111.6 \pm 1.1$ & $5120 \pm 290 \times 10^{23}$ & $1.32 \pm 0.12$ & $0.19 \pm 0.01$ & $0.30 \pm 0.07$ \\
\hline 2015/11/01.02-01.46 & 1.568 & -36.10 & $171.2 \pm 1.8$ & $5555 \pm 446 \times 10^{23}$ & $2.07 \pm 0.17$ & $0.29 \pm 0.02$ & $0.41 \pm 0.08$ \\
\hline & & 5.90 & & $60 \pm 920 \times 10^{23}$ & \pm 0.39 & $0.26 \pm$ & $0.54 \pm 0.12$ \\
\hline & & -28.71 & & $00 \times 10^{23}$ & 0.38 & 0.1 & $0.68 \pm$ \\
\hline $2015 / 11 / 22.02-22.51$ & 1.718 & -28.55 & & $2280 \pm 100 \times 10^{23}$ & $3.20 \pm 0.54$ & $0.12 \pm 0.01$ & $0.65 \pm 0.10$ \\
\hline 2015/12/03.04-03.98 & 1.801 & -24.83 & $27.0 \pm 2.5$ & $674 \pm 131 \times 10^{23}$ & $1.66 \pm 0.33$ & $0.19 \pm 0.04$ & $0.0 \pm 0.94$ \\
\hline & & -21.82 & & $4 \times 10^{23}$ & $=0.67$ & 01 & $0.72 \pm 0.16$ \\
\hline 2016/01/02.24-02.83 & 2.03 & -16.10 & & $30 \times 10^{23}$ & $3.62 \pm 0.37$ & $0.10 \pm 0.01$ & $0.73 \pm 0.17$ \\
\hline $2016 / 01 / 27.52-28.49$ & 2.228 & -9.96 & $161.3 \pm 0.5$ & $567 \pm 27 \times 10^{23}$ & $3.10 \pm 0.28$ & $0.13 \pm 0.01$ & $0.58 \pm 0.19$ \\
\hline 2016/01/29.02-29.99 & 2.241 & -9.62 & $152.0 \pm 5.3$ & $555 \pm 28 \times 10^{23}$ & $3.28 \pm 0.32$ & $0.12 \pm 0.01$ & $0.65 \pm 0.24$ \\
\hline & & & & $490 \pm 22 \times 10^{23}$ & & $08 \pm 0.01$ & $1.06 \pm 0.40$ \\
\hline $2016 / 02 / 02.54-03.45$ & & -8.67 & & $16 \times 10^{23}$ & $2.76 \pm 0.40$ & $0.09 \pm 0.02$ & $0.31 \pm 0$ \\
\hline 2016/02/04.03-04.99 & 2.286 & -8.33 & $110.1 \pm 8.1$ & $455 \pm 23 \times 10^{23}$ & $3.12 \pm 0.20$ & $0.08 \pm 0.01$ & $0.94 \pm 0.32$ \\
\hline 2016/02/06.12-06.52 & 2.298 & -7.95 & $141.7 \pm 2.3$ & $458 \pm 26 \times 10^{23}$ & $3.47 \pm 0.38$ & $0.08 \pm 0.01$ & $0.0 \pm 0.45$ \\
\hline 2016/02/06.52-07.01 & 2.30 & -7.86 & $146.9 \pm 2.8$ & $434 \pm 38 \times 10^{23}$ & & $0.09 \pm 0.02$ & \\
\hline $06.52-08.49$ & & -7.40 & & $431 \pm 9 \times 10^{23}$ & $3.48 \pm$ & $0.09 \pm 0.01$ & $0.82 \pm 0.26$ \\
\hline 2016/02/15.00-17.01 & 2.37 & -5.98 & $143 \pm 15$ & $392 \pm 2 \times 10^{23}$ & $3.55 \pm 0.48$ & $0.10 \pm 0.01$ & $0.43 \pm 0.29$ \\
\hline $2016 / 02 / 23.03-25.99$ & 2.436 & -4.35 & $18 \pm 13$ & $49 \pm 4 \times 10^{23}$ & $2.13 \pm 1.12$ & $0.38 \pm 0.33$ & $0.0 \pm 4.0$ \\
\hline & & -0.03 & & $69 \pm 3.5 \times 10^{23}$ & $7.26 \pm 1.28$ & $0.32 \pm 0.12$ & $8.3 \pm 3.4$ \\
\hline & & & & $22 \pm 1.6 \times 10^{23}$ & $12.1 \pm 1.3$ & $0.0 \pm 0.33$ & $0.0 \pm 6.5$ \\
\hline 2016/06/11.11-12.99 & 3.187 & +11.03 & $159 \pm 13$ & $12.7 \pm 0.7 \times 10^{23}$ & $22.8 \pm 4.9$ & $0.0 \pm 0.83$ & $0.0 \pm 14.6$ \\
\hline $2016 / 06 / 14.05-16.01$ & 3.211 & +11.35 & $97 \pm 25$ & $13.5 \pm 1.9 \times 10^{23}$ & $24.4 \pm 10.2$ & $0.0 \pm 1.01$ & $0.0 \pm 11.1$ \\
\hline 2016/06/18.01-19.99 & 3.231 & +11.77 & $15 \pm 8$ & $7.0 \pm 1.1 \times 10^{23}$ & $20.0 \pm 7.8$ & $0.0 \pm 23.3$ & $0.0 \pm 37.3$ \\
\hline \multicolumn{8}{|l|}{ Average over a Rosett } \\
\hline & & +0 & & $956 \pm 43 \times 10^{23}$ & & & \\
\hline 2015/05/16.01-30.00 & 1.580 & -5.4 & $29-151$ & $1568 \pm 60 \times 10^{23}$ & $0.75 \pm 0.07$ & $0.62 \pm 0.02$ & $0.11 \pm 0.03$ \\
\hline
\end{tabular}

Notes. ${ }^{(a)}$ Latitude of the subsolar point. ${ }^{(b)}$ Angle between the north pole and the direction of Rosetta. 
Appendix B: Maps of $\mathrm{H}_{2}^{18} \mathrm{O}$ emission (and $\mathrm{H}_{2}^{16} \mathrm{O}$ on 29 July 2014)
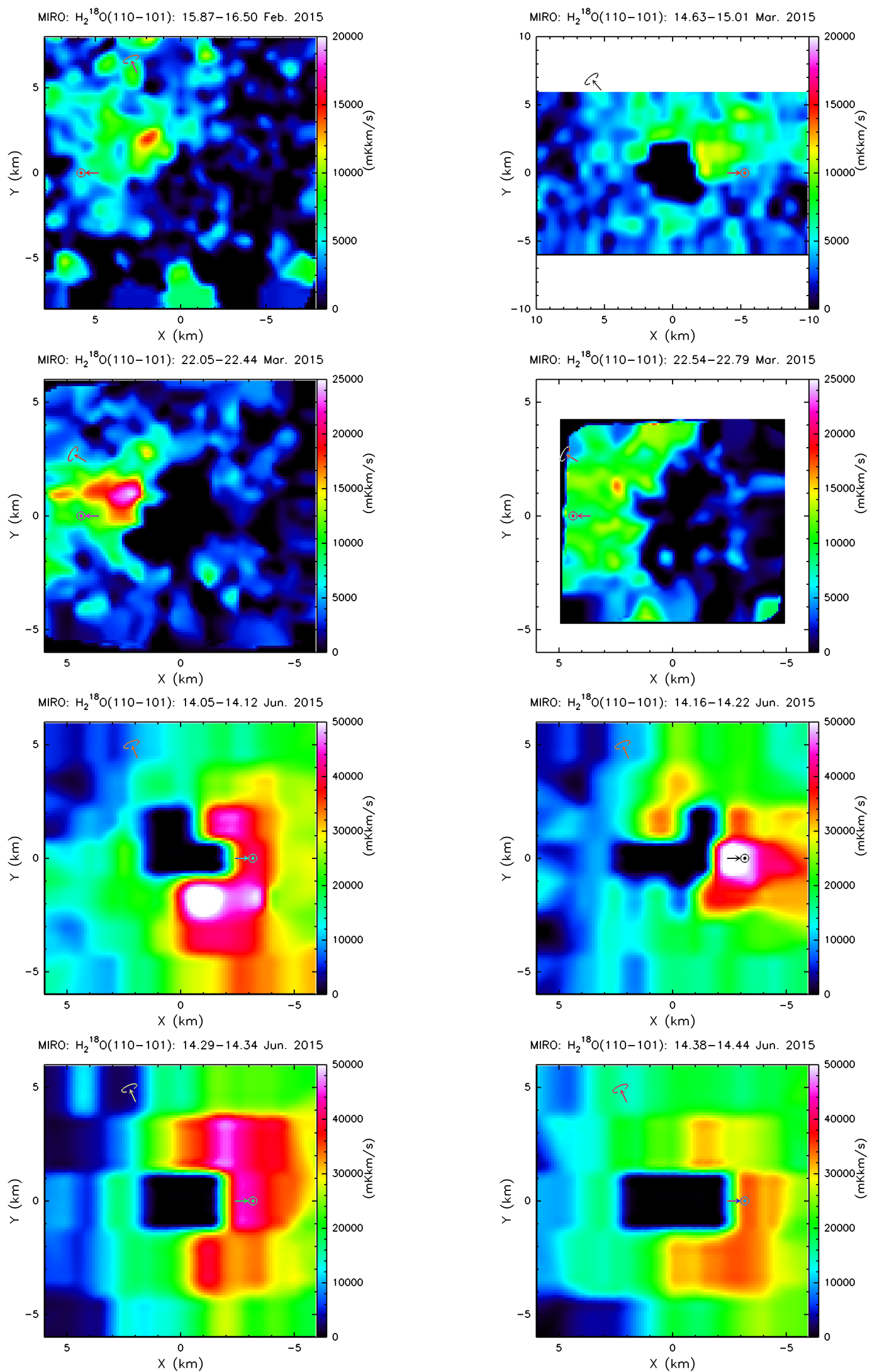

Fig. B.1. Maps of interpolated $\mathrm{H}_{2}^{18} \mathrm{O}$ line intensity on 16 February, 14 and $22 \mathrm{March}$, and 14 June 2015. 

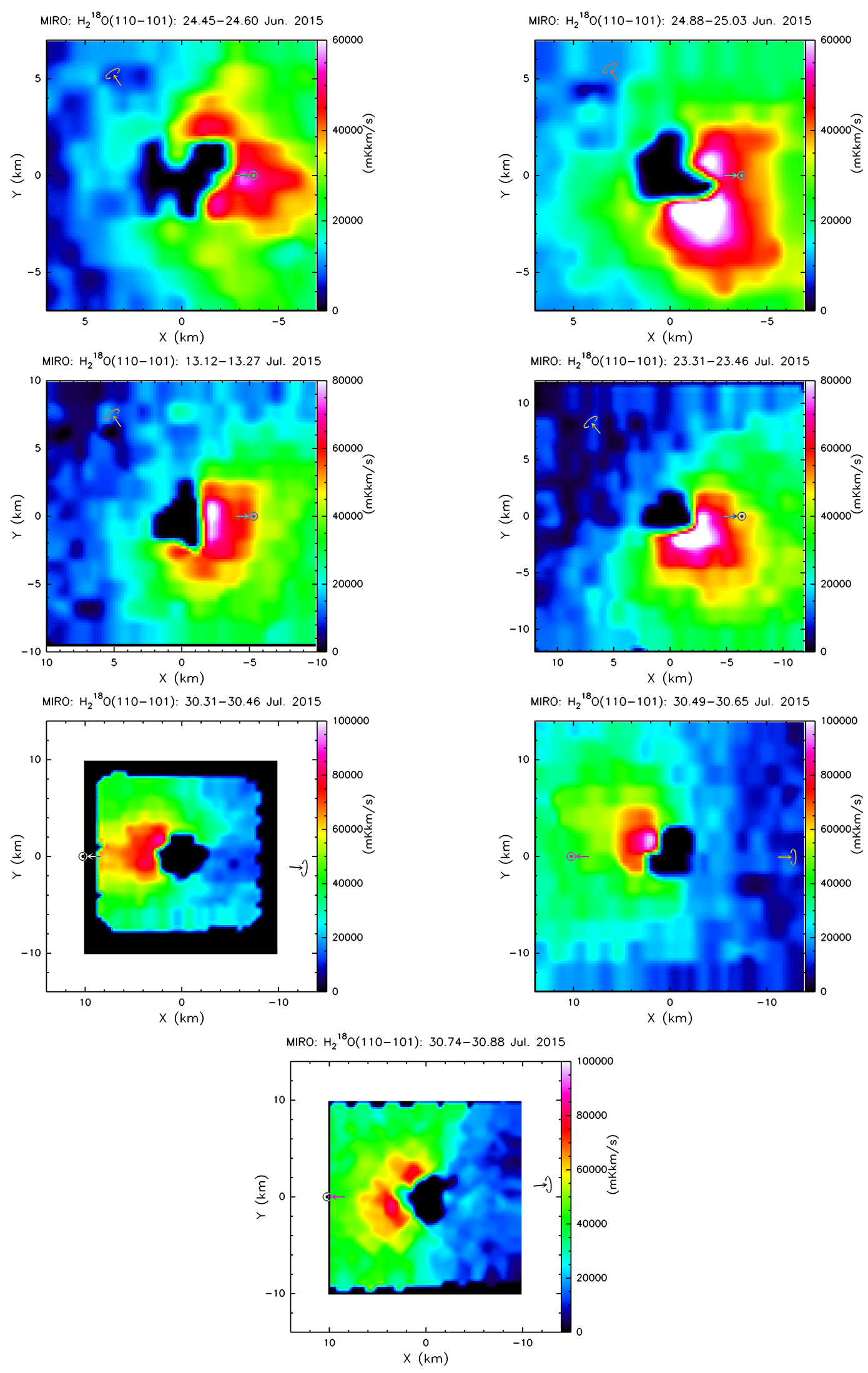

Fig. B.2. Maps of interpolated $\mathrm{H}_{2}^{18} \mathrm{O}$ line intensity on 24 June and 13, 23, and 30 July 2015. 

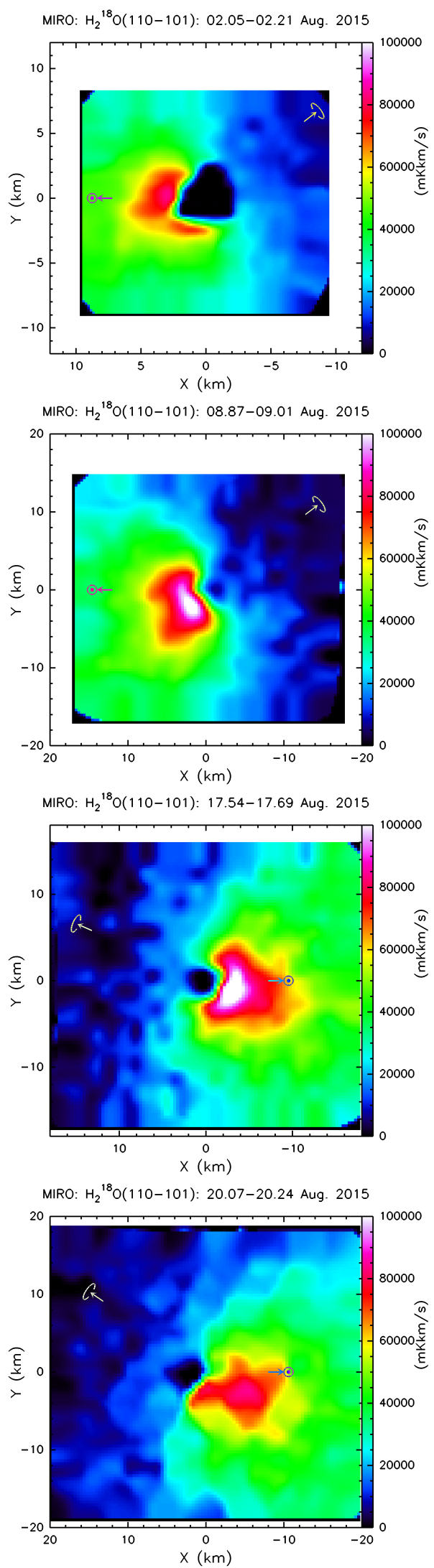

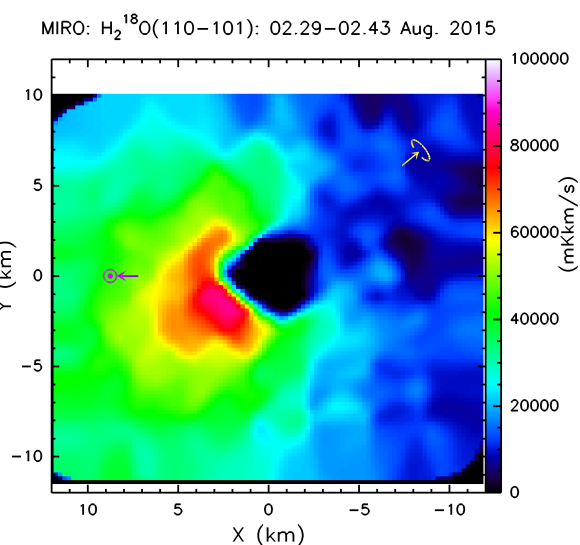

MIRO: $\mathrm{H}_{2}{ }^{18} \mathrm{O}(110-101)$ : 09.05-09.21 Aug. 2015

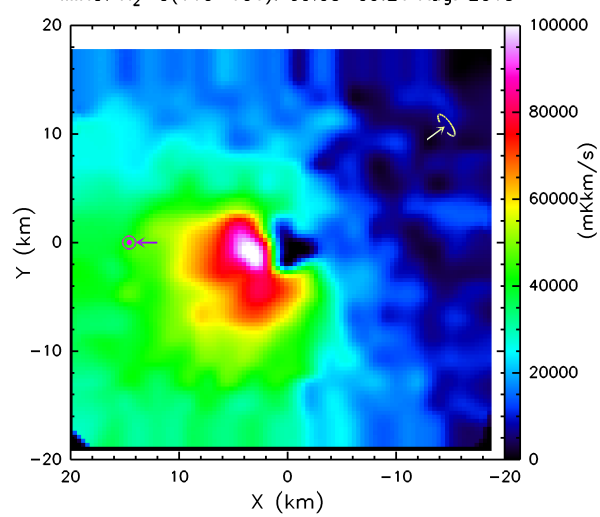

MIRO: $\mathrm{H}_{2}{ }^{18} \mathrm{O}(110-101): 17.72-17.86$ Aug. 2015

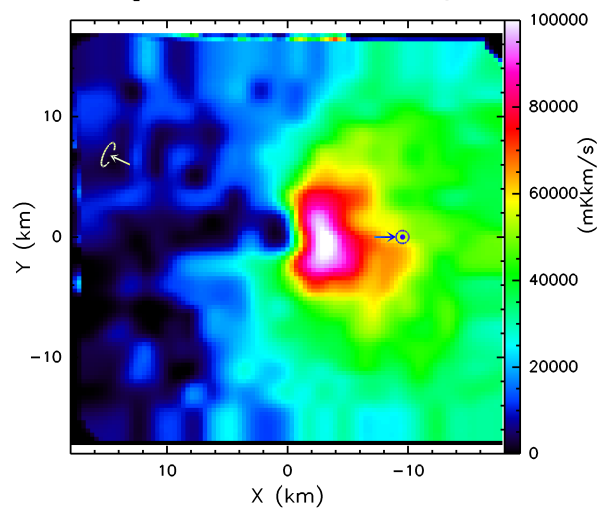

MIRO: $\mathrm{H}_{2}{ }^{18} \mathrm{O}(110-101): 20.31-20.43$ Aug. 2015

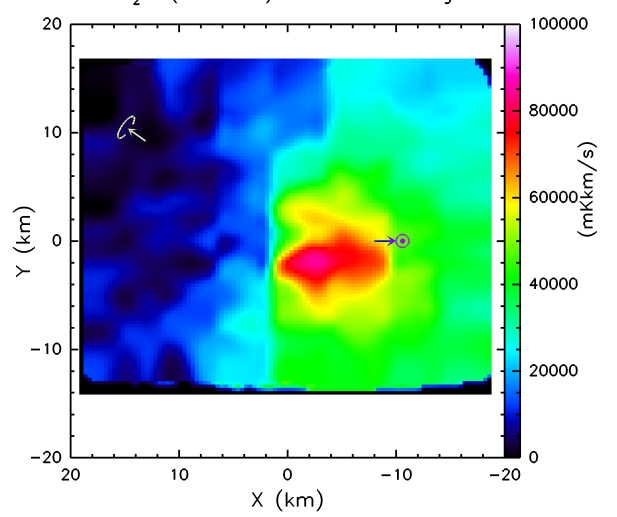

Fig. B.3. Maps of interpolated $\mathrm{H}_{2}^{18} \mathrm{O}$ line intensity on 2, 8, 9, 17, and 20 August 2015 . 

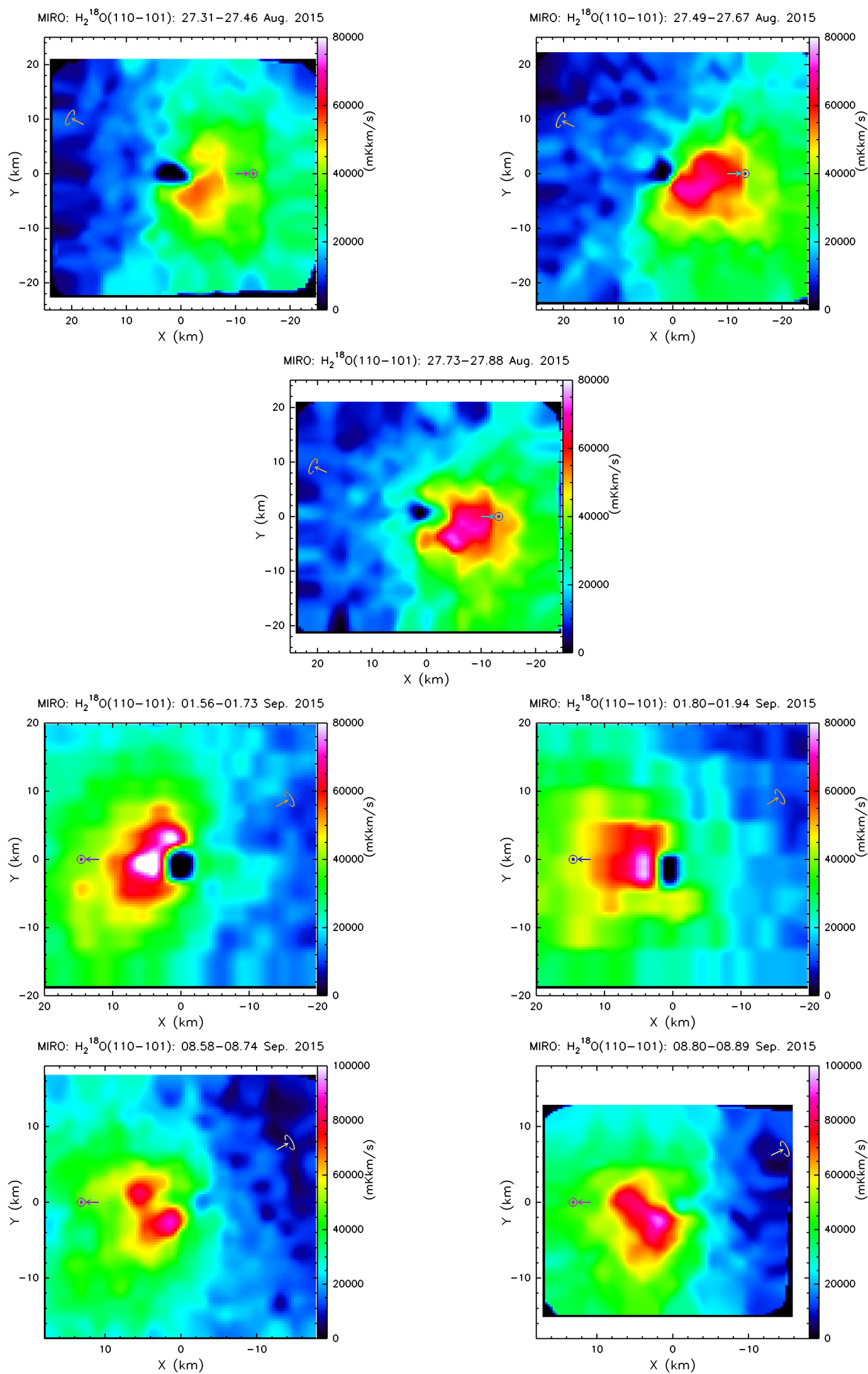

Fig. B.4. Maps of interpolated $\mathrm{H}_{2}^{18} \mathrm{O}$ line intensity on 27 August and 1 and 8 September 2015. 
N. Biver et al.: Monitoring of comet 67P with Rosetta/MIRO
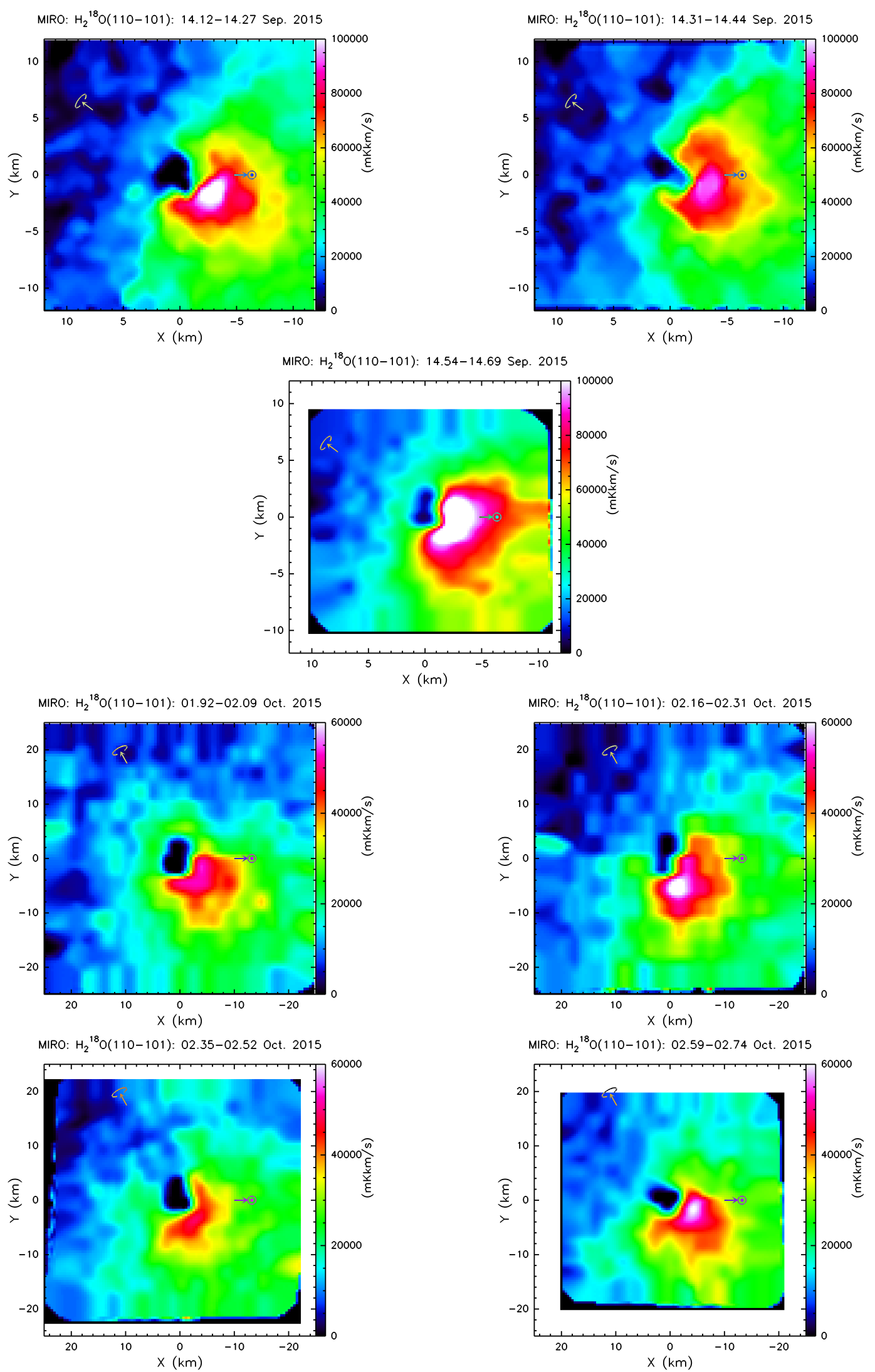

Fig. B.5. Maps of interpolated $\mathrm{H}_{2}^{18} \mathrm{O}$ line intensity on 14 September and 2 October 2015. 


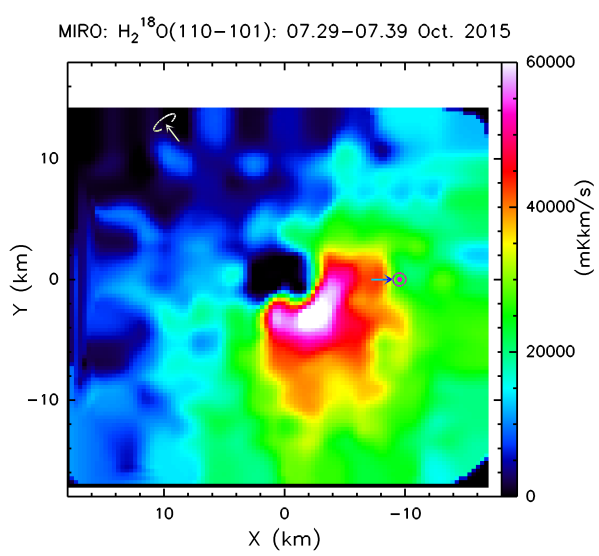

MIRO: $\mathrm{H}_{2}{ }^{18} \mathrm{O}(110-101): 07.88-07.93$ Oct. 2015

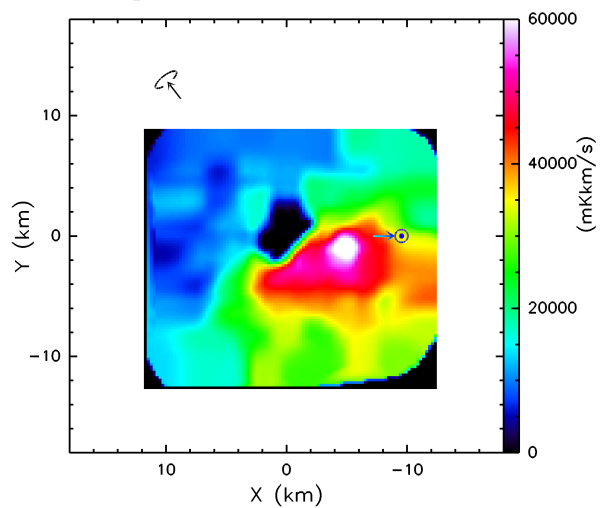

MIRO: $H_{2}{ }^{18}$ O(110-101): $17.28-17.39$ Oct. 2015

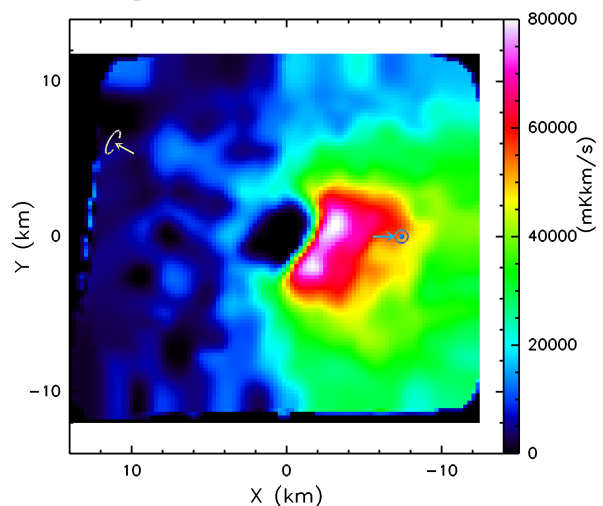

MIRO: $\mathrm{H}_{2}{ }^{18} \mathrm{O}(110-101): 07.46-07.60$ Oct. 2015

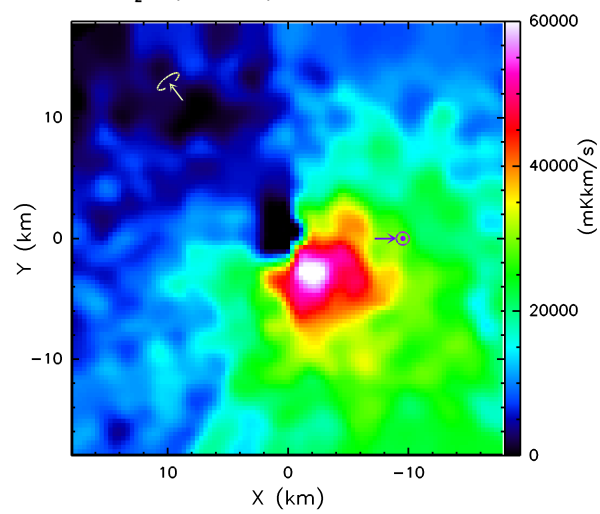

MIRO: $H_{2}{ }^{18} \mathrm{O}(110-101): 07.98-08.03$ Oct. 2015

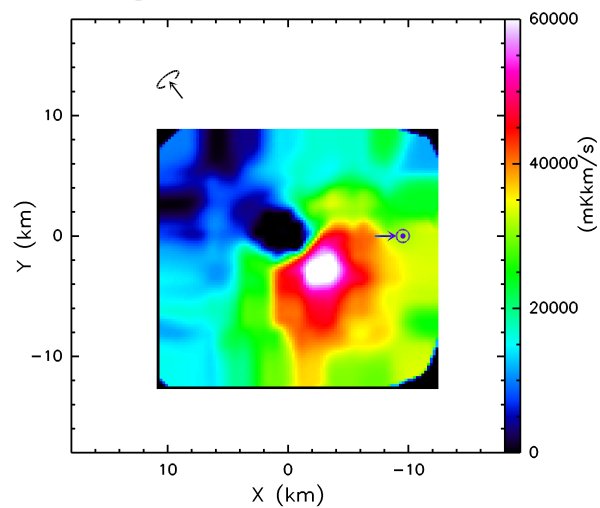

MIRO: $\mathrm{H}_{2}{ }^{18}$ O(110-101): $17.45-17.59$ Oct. 2015

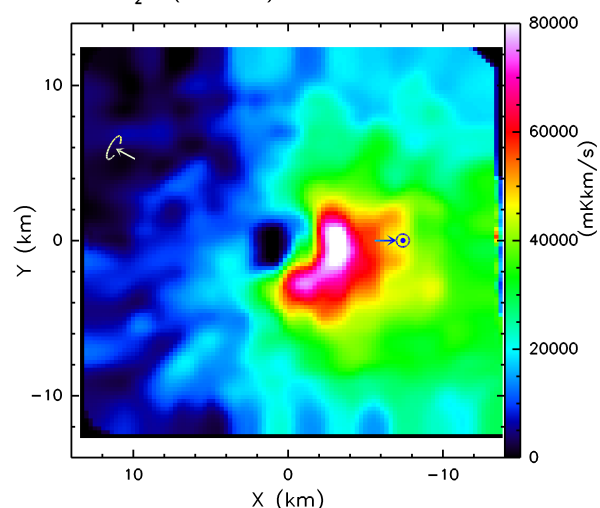

MIRO: $\mathrm{H}_{2}{ }^{18} \mathrm{O}(110-101): 17.63-17.79$ Oct. 2015

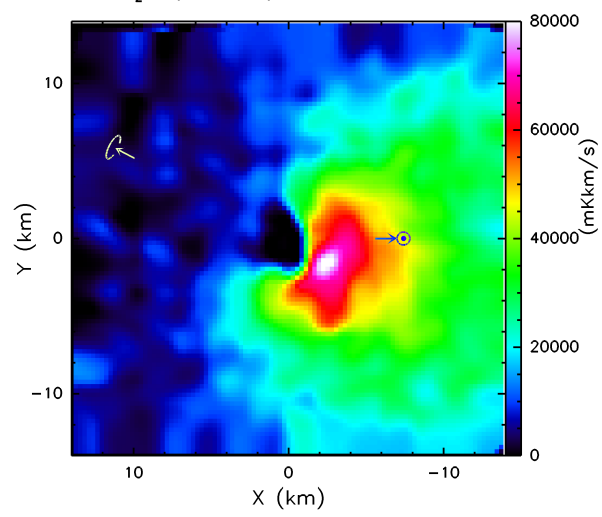

Fig. B.6. Maps of interpolated $\mathrm{H}_{2}^{18} \mathrm{O}$ line intensity on 14 September and 2 October 2015. 
N. Biver et al.: Monitoring of comet $67 \mathrm{P}$ with Rosetta/MIRO
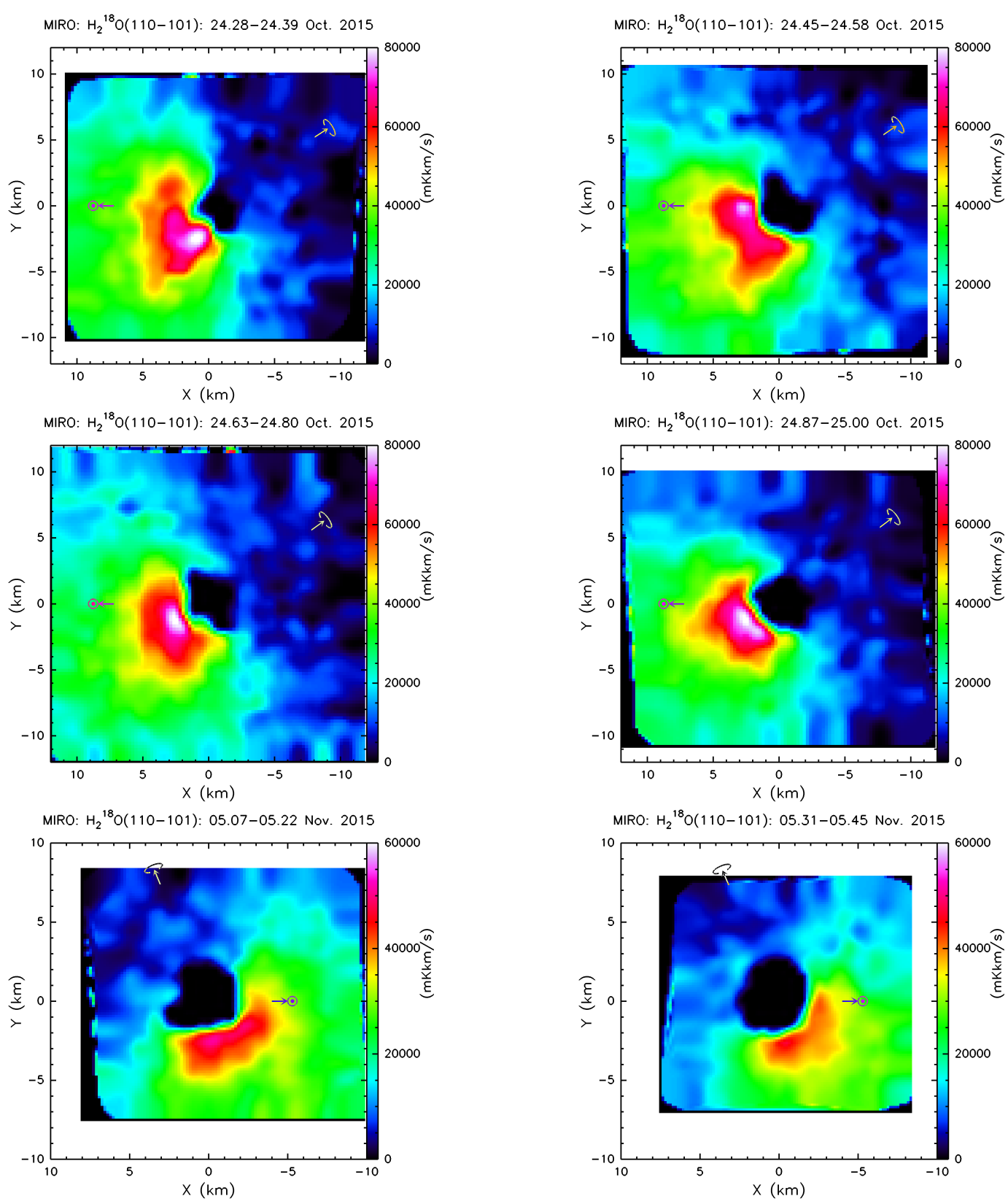

MIRO: $\mathrm{H}_{2}{ }^{18} \mathrm{O}(110-101)$ : 05.49-05.66 Nov. 2015
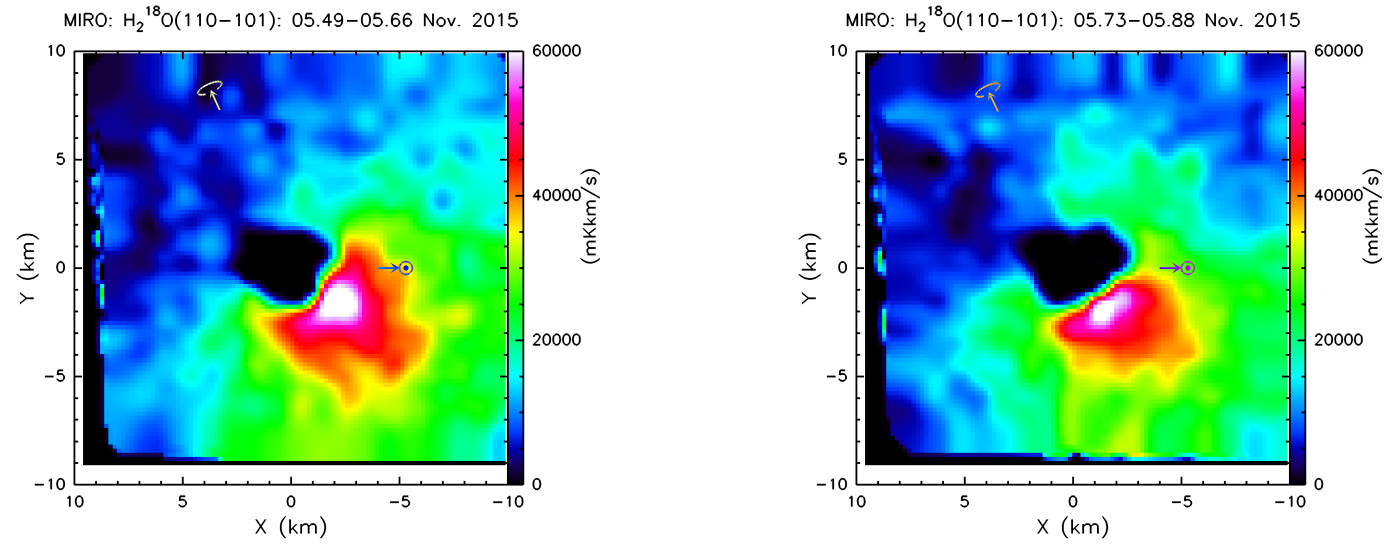

Fig. B.7. Maps of interpolated $\mathrm{H}_{2}^{18} \mathrm{O}$ line intensity on 24 October and 5 November 2015. 
A\&A 630, A19 (2019)

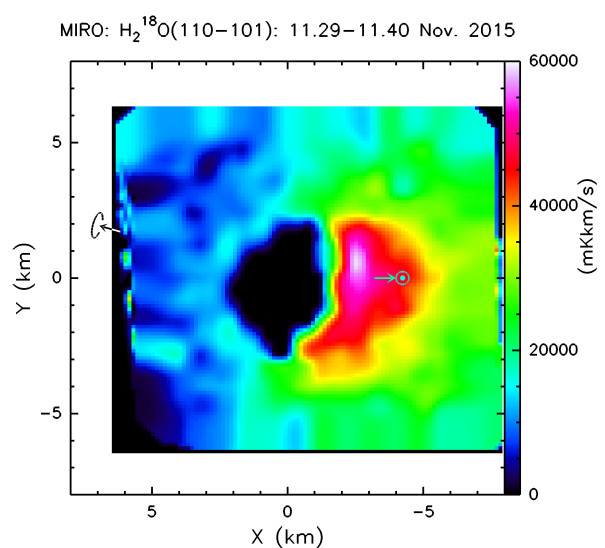

MIRO: $\mathrm{H}_{2}{ }^{18} \mathrm{O}(110-101): 11.64-11.82$ Nov. 2015

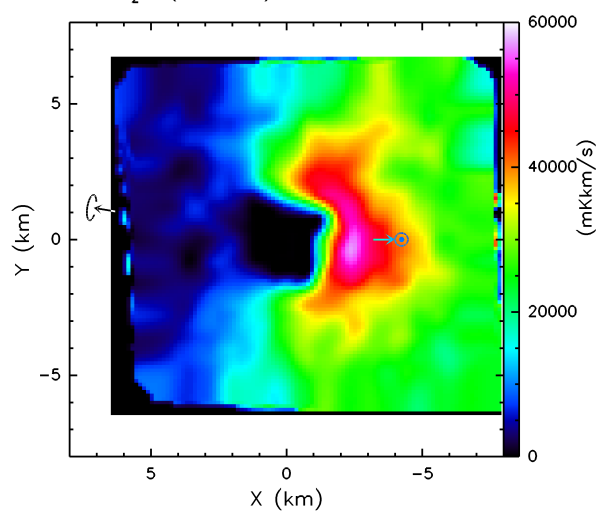

MIRO: $\mathrm{H}_{2}{ }^{18} \mathrm{O}(110-101): 15.49-15.64$ Nov. 2015

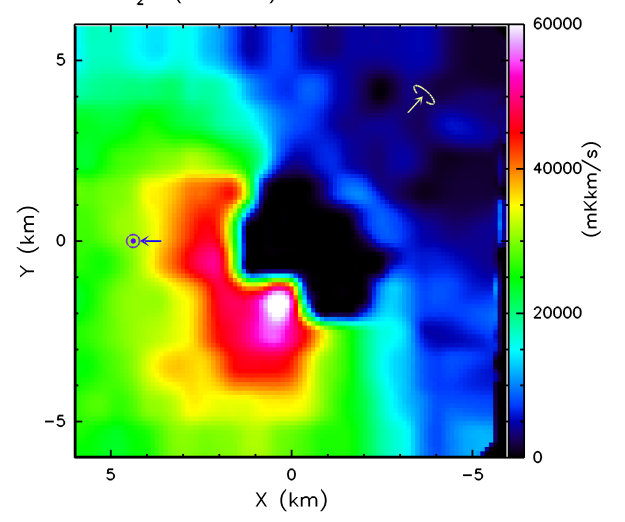

MIRO: $\mathrm{H}_{2}{ }^{18} \mathrm{O}(110-101): 11.45-11.61$ Nov. 2015

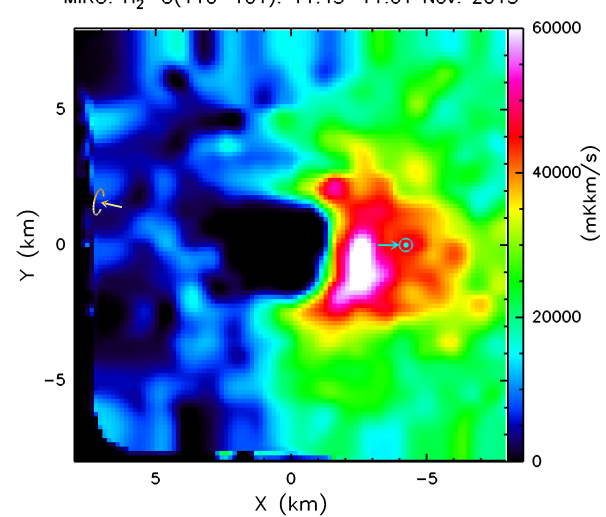

MIRO: $\mathrm{H}_{2}{ }^{18} \mathrm{O}(110-101): 15.29-15.44$ Nov. 2015

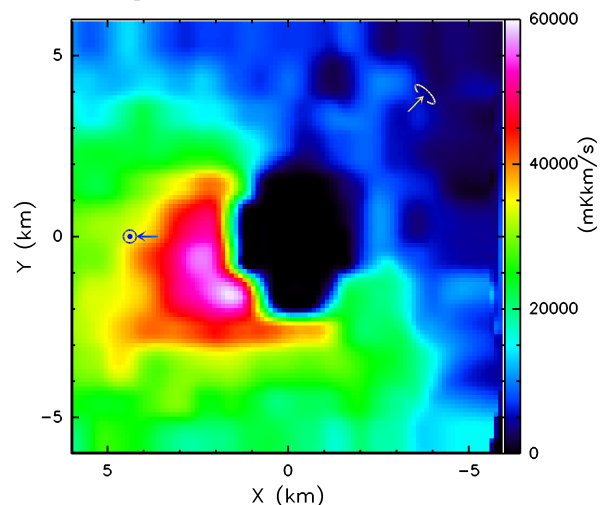

MIRO: $\mathrm{H}_{2}{ }^{18} \mathrm{O}(110-101)$ : $15.71-15.85$ Nov. 2015

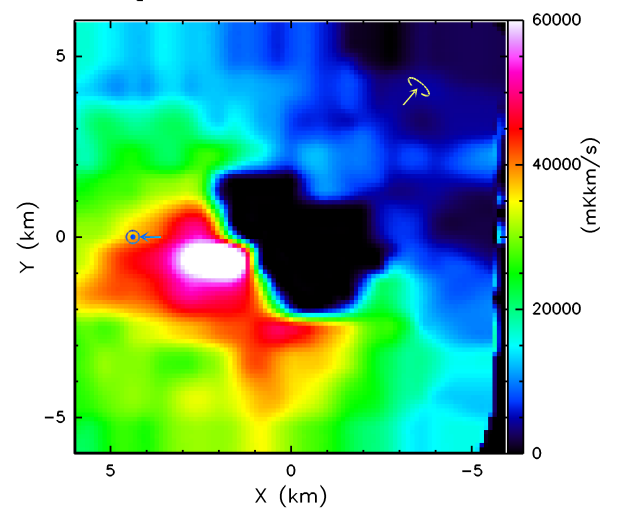

Fig. B.8. Maps of interpolated $\mathrm{H}_{2}^{18} \mathrm{O}$ line intensity on 11 and 15 November 2015 . 

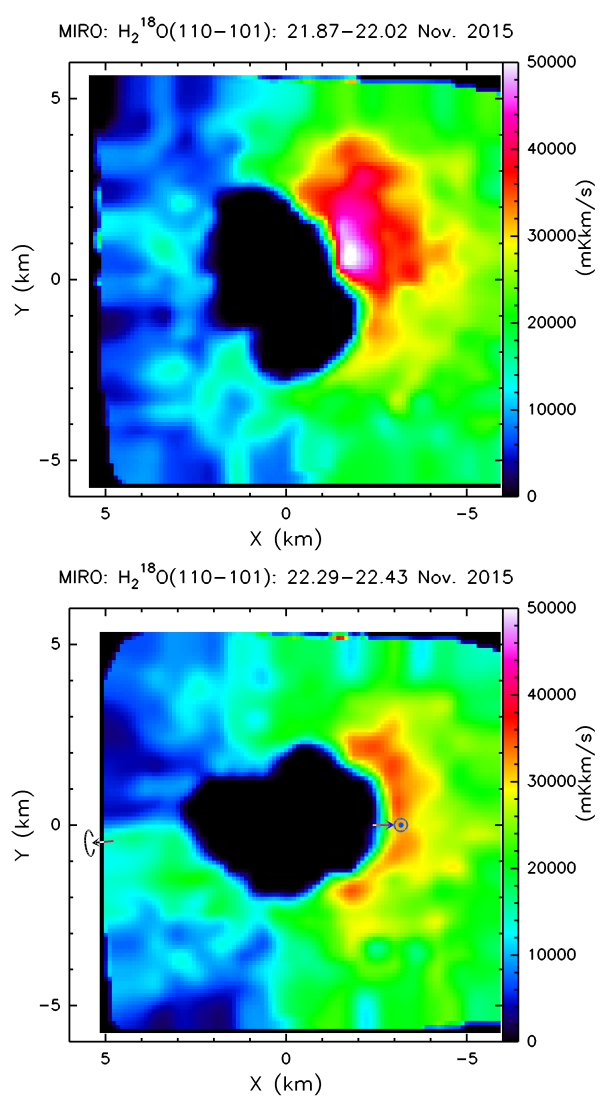

MIRO: $\mathrm{H}_{2}{ }^{18} \mathrm{O}(110-101): 26.31-26.46$ Nov. 2015

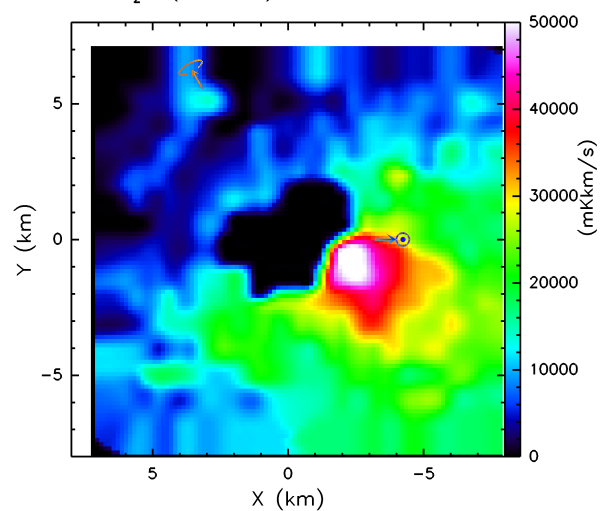

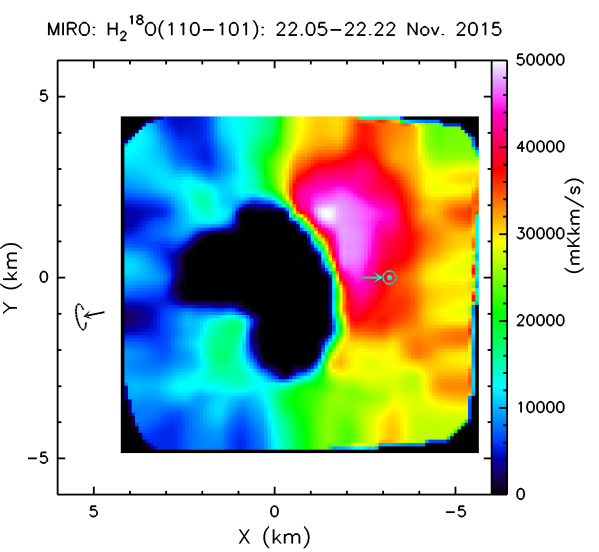

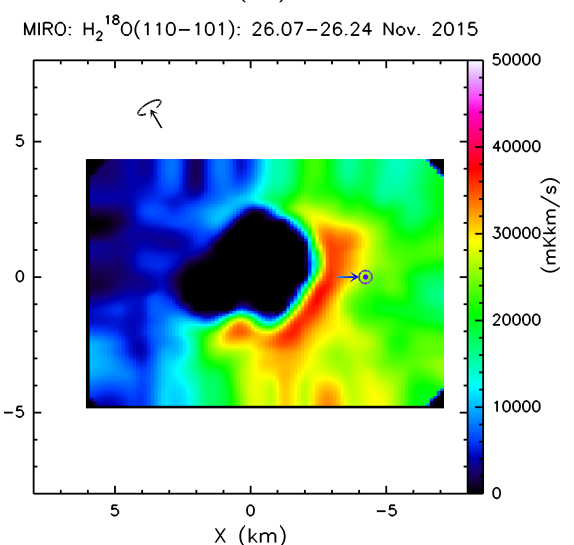

MIRO: $\mathrm{H}_{2}{ }^{18} \mathrm{O}(110-101): 26.49-26.67$ Nov. 2015
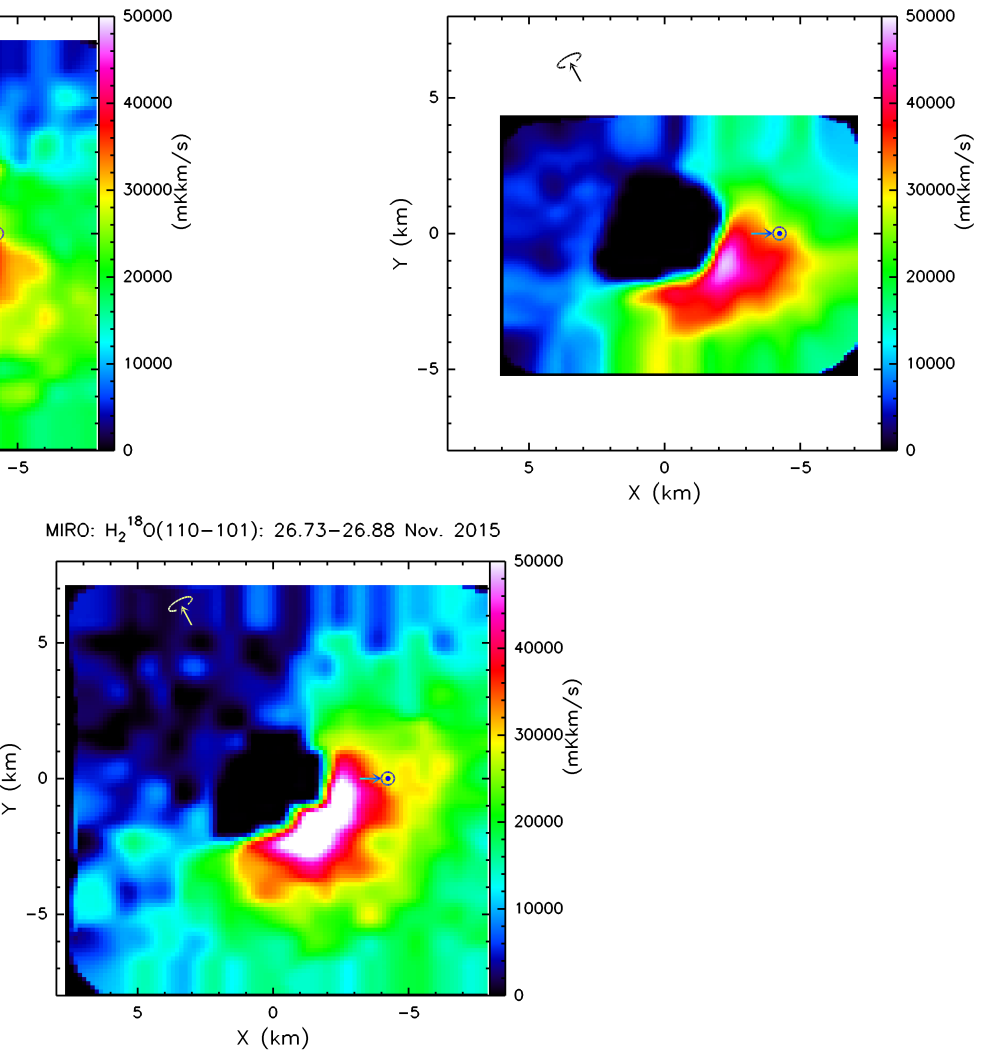

Fig. B.9. Maps of interpolated $\mathrm{H}_{2}^{18} \mathrm{O}$ line intensity on 22 and 26 November 2015. 


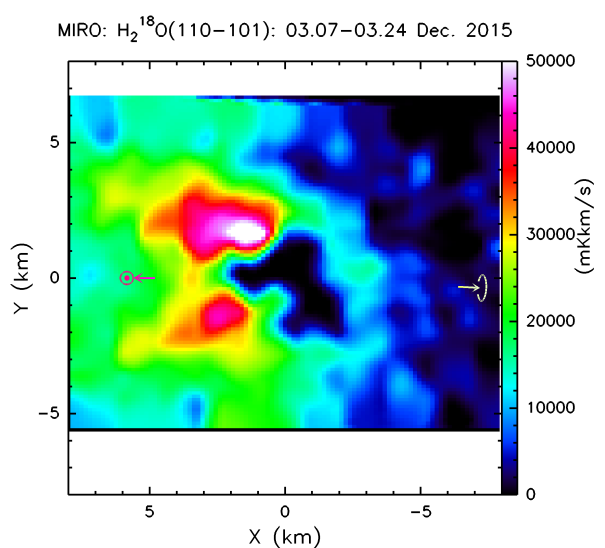

MIRO: $\mathrm{H}_{2}{ }^{18} \mathrm{O}(110-101): 03.49-03.67$ Dec. 2015

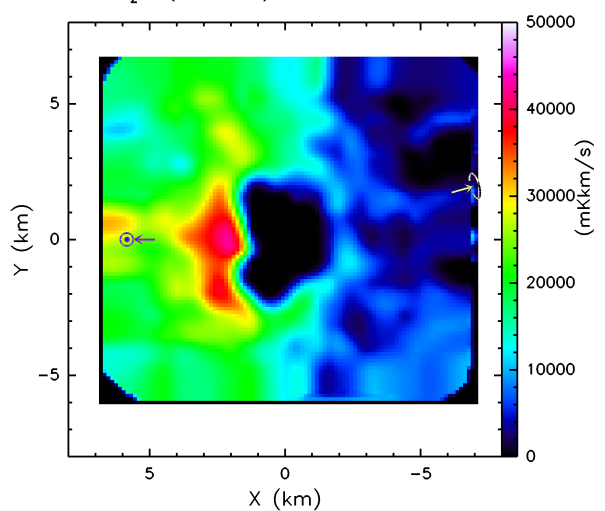

MIRO: $\mathrm{H}_{2}{ }^{18} \mathrm{O}(110-101): 22.61-22.74$ Dec. 2015
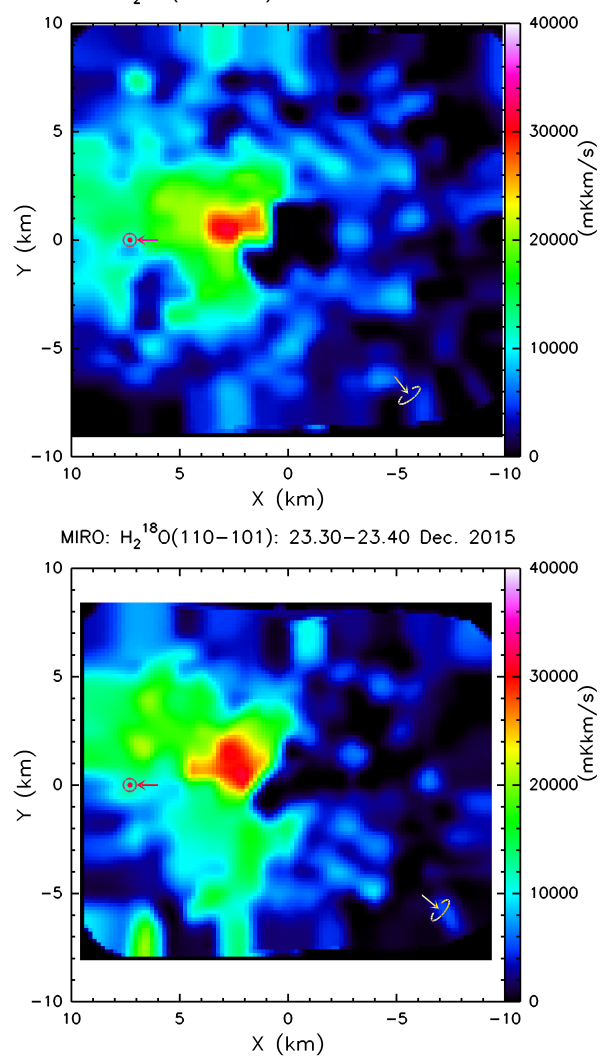

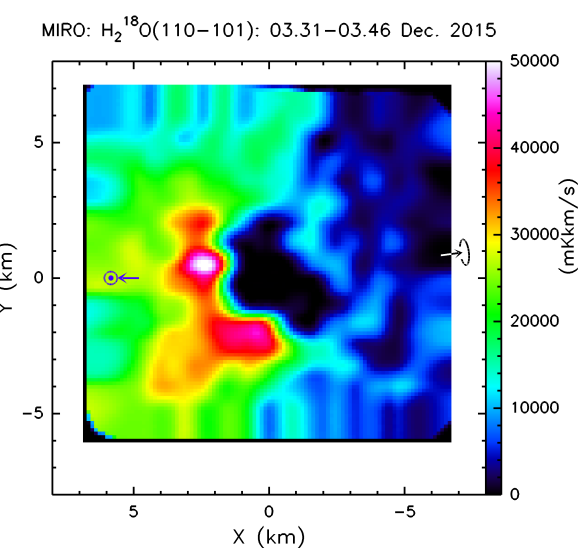

MIRO: $H_{2}{ }^{18} \mathrm{O}(110-101): 03.73-03.88$ Dec. 2015

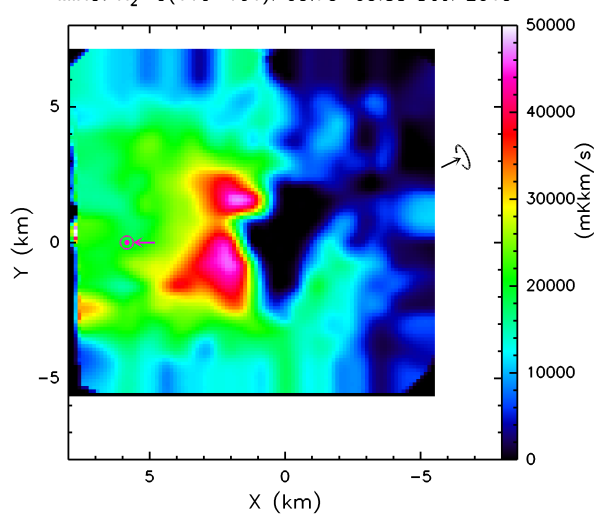

MIRO: $\mathrm{H}_{2}{ }^{18} \mathrm{O}(110-101): 22.80-22.94$ Dec. 2015

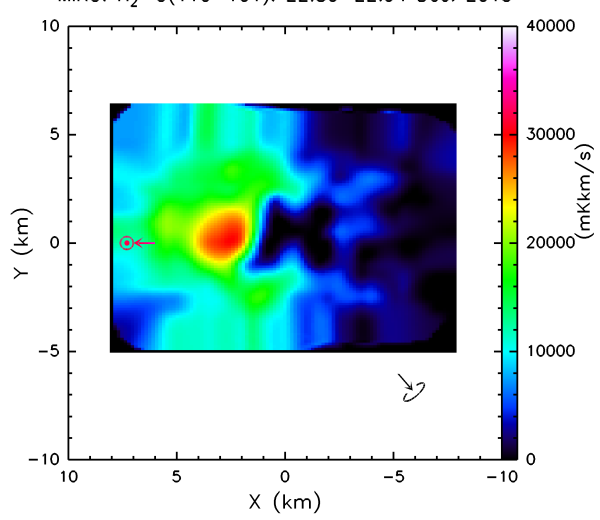

MIRO: $H_{2}{ }^{18} \mathrm{O}(110-101): 23.64-23.81$ Dec. 2015

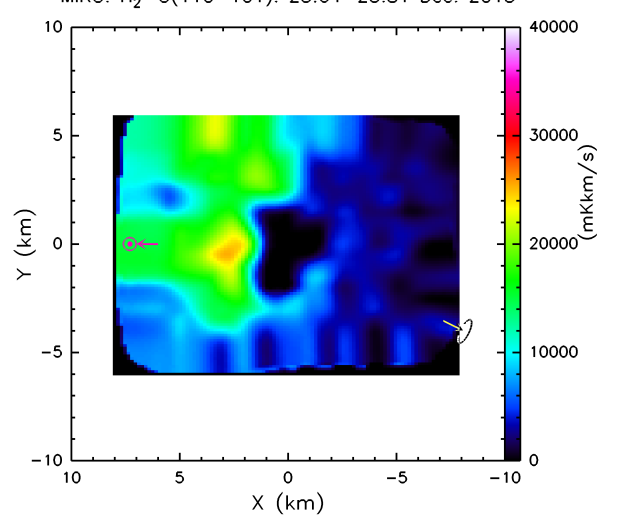

Fig. B.10. Maps of interpolated $\mathrm{H}_{2}^{18} \mathrm{O}$ line intensity on 3, 22, and 23 December 2015. 


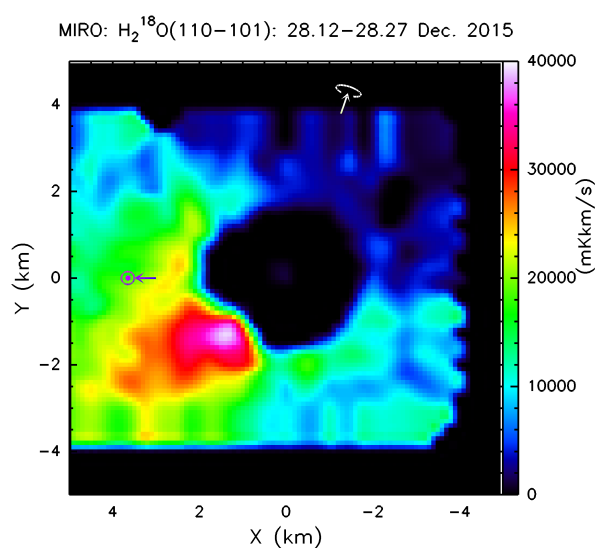

MIRO: $H_{2}{ }^{18} \mathrm{O}(110-101): 28.54-28.69$ Dec. 2015

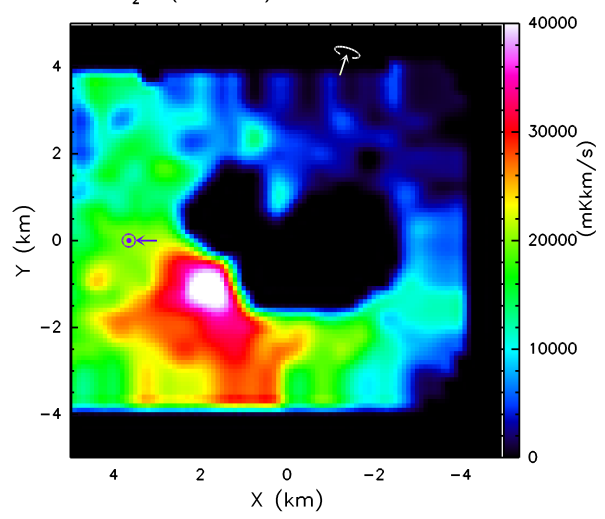

MIRO: $\mathrm{H}_{2}{ }^{18} \mathrm{O}(110-101): 31.31-31.46$ Dec. 2015
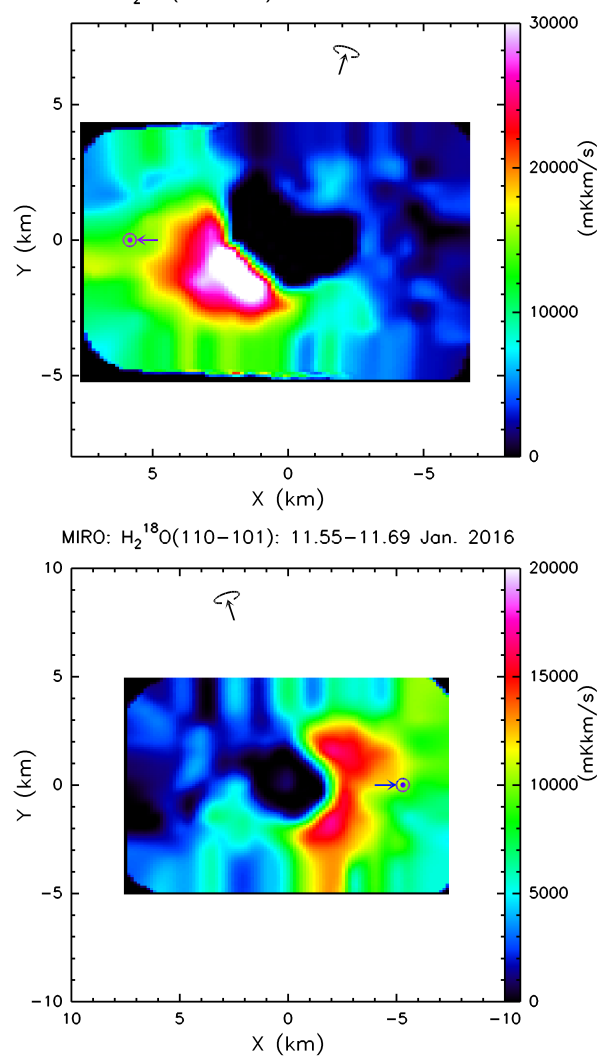

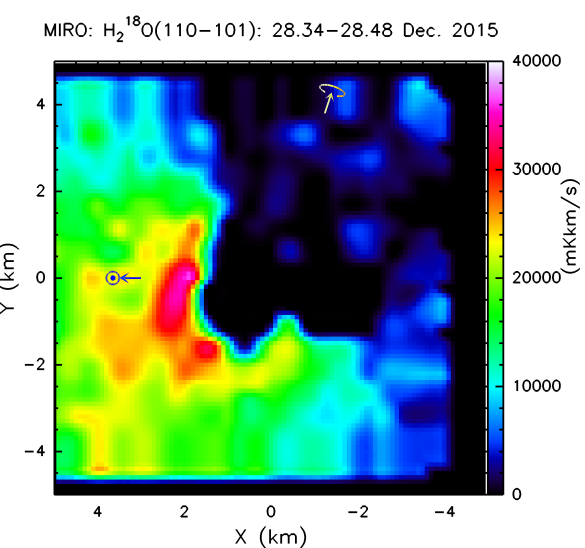

MIRO: $\mathrm{H}_{2}{ }^{18} \mathrm{O}(110-101)$ : 31.07-31.24 Dec. 2015

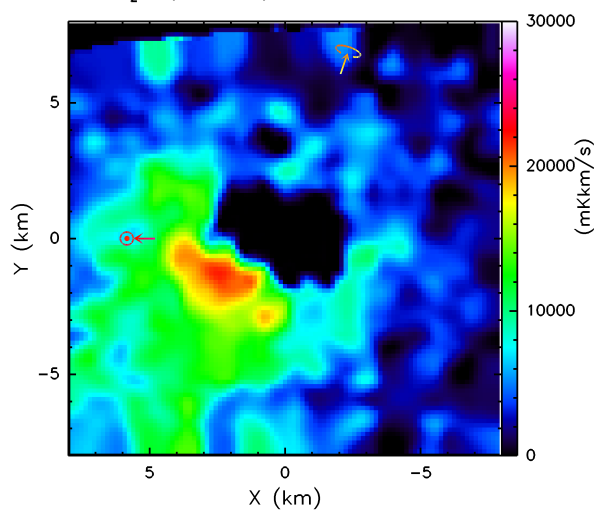

MIRO: $H_{2}^{18} O(110-101): 31.49-31.67$ Dec. 2015

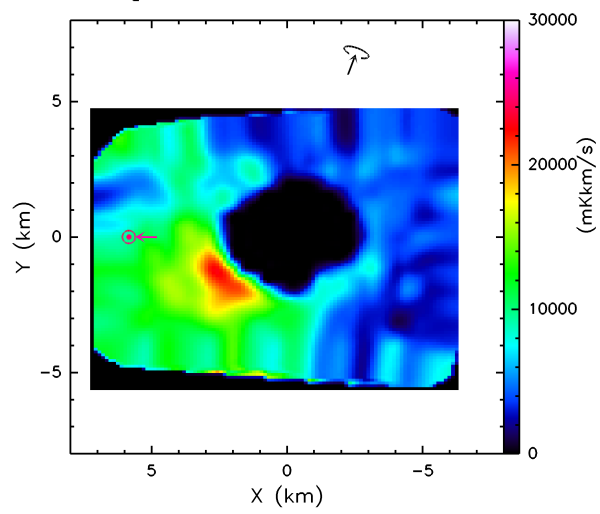

MIRO: $\mathrm{H}_{2}{ }^{18} \mathrm{O}(110-101): 12.80-12.94$ Jan. 2016

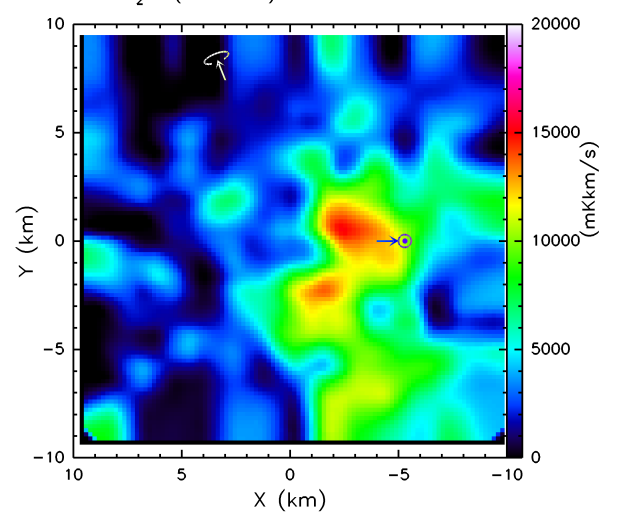

Fig. B.11. Maps of interpolated $\mathrm{H}_{2}^{18} \mathrm{O}$ line intensity on 28 and 31 December 2015 and 11 and 12 January 2016. 

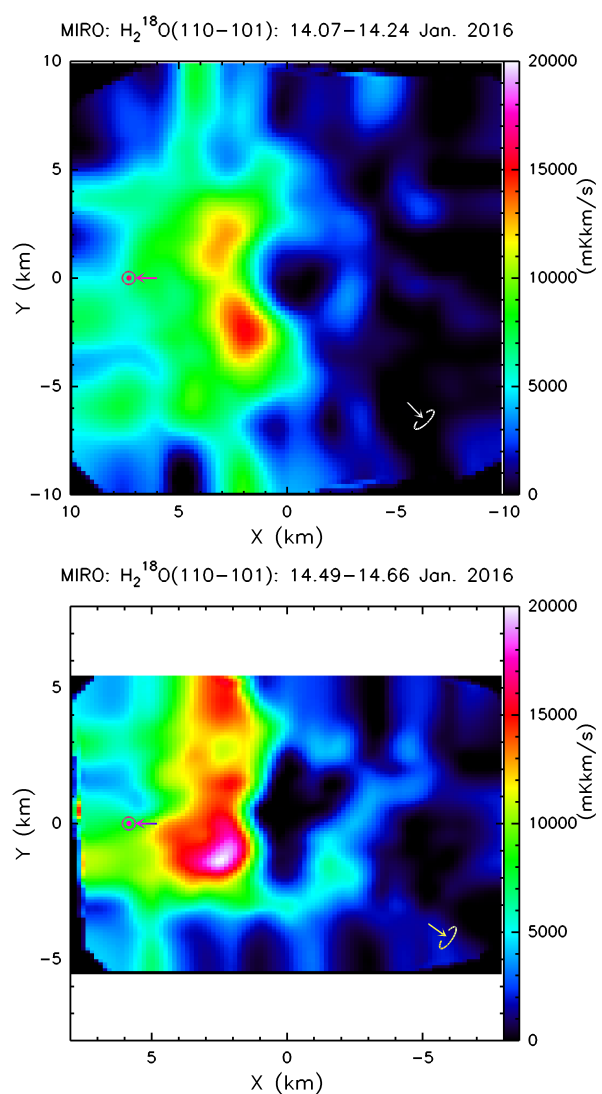

MIRO: $\mathrm{H}_{2}{ }^{18} \mathrm{O}(110-101):$ 14.92-15.09 Jan. 2016

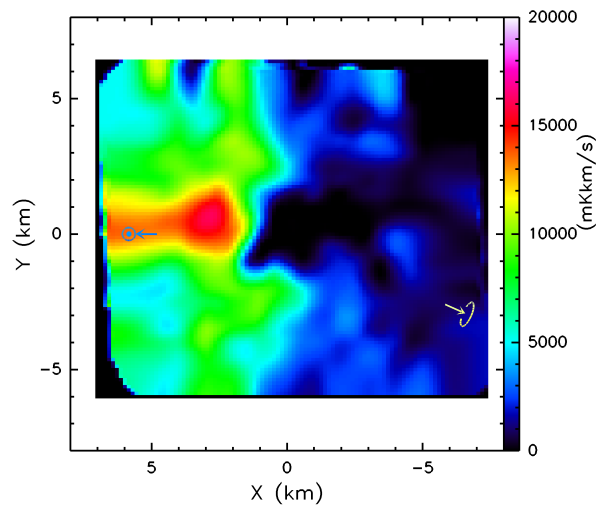

MIRO: $\mathrm{H}_{2}{ }^{18} \mathrm{O}(110-101): 14.31-14.46$ Jan. 2016

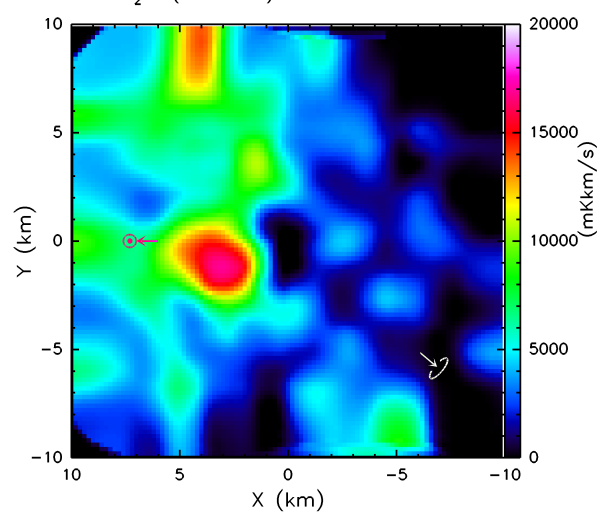

MIRO: $\mathrm{H}_{2}{ }^{18} \mathrm{O}(110-101): 14.73-14.88$ Jan. 2016

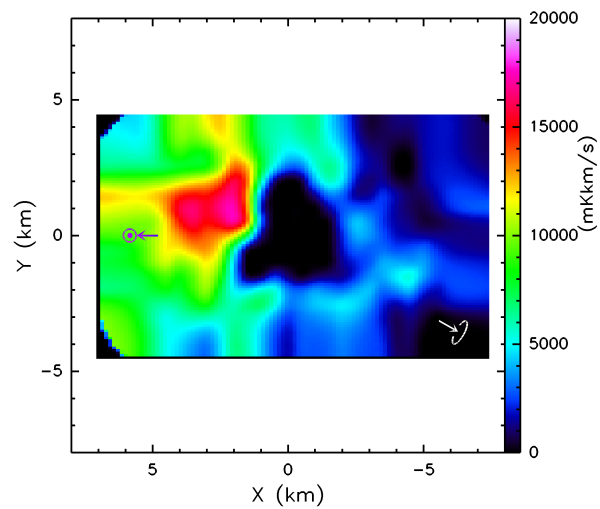

MIRO: $\mathrm{H}_{2}{ }^{18} \mathrm{O}(110-101): 15.16-15.31$ Jan. 2016

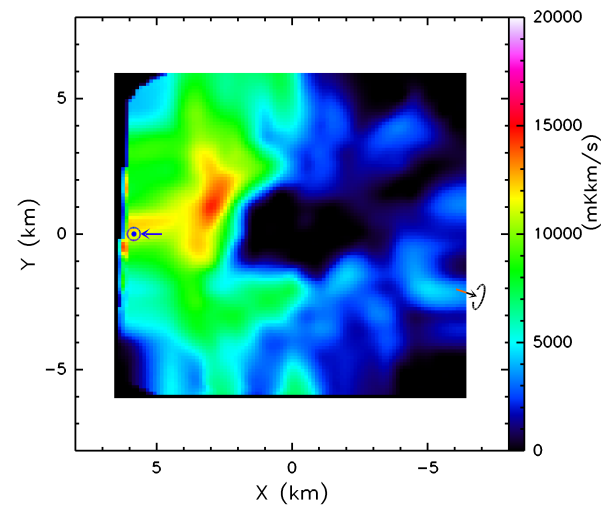

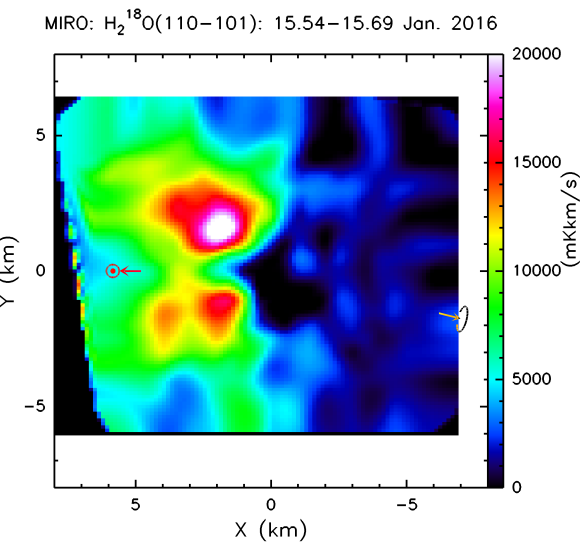

Fig. B.12. Maps of interpolated $\mathrm{H}_{2}^{18} \mathrm{O}$ line intensity on 11, 12, 14, and 15 January 2016. 
N. Biver et al.: Monitoring of comet 67P with Rosetta/MIRO
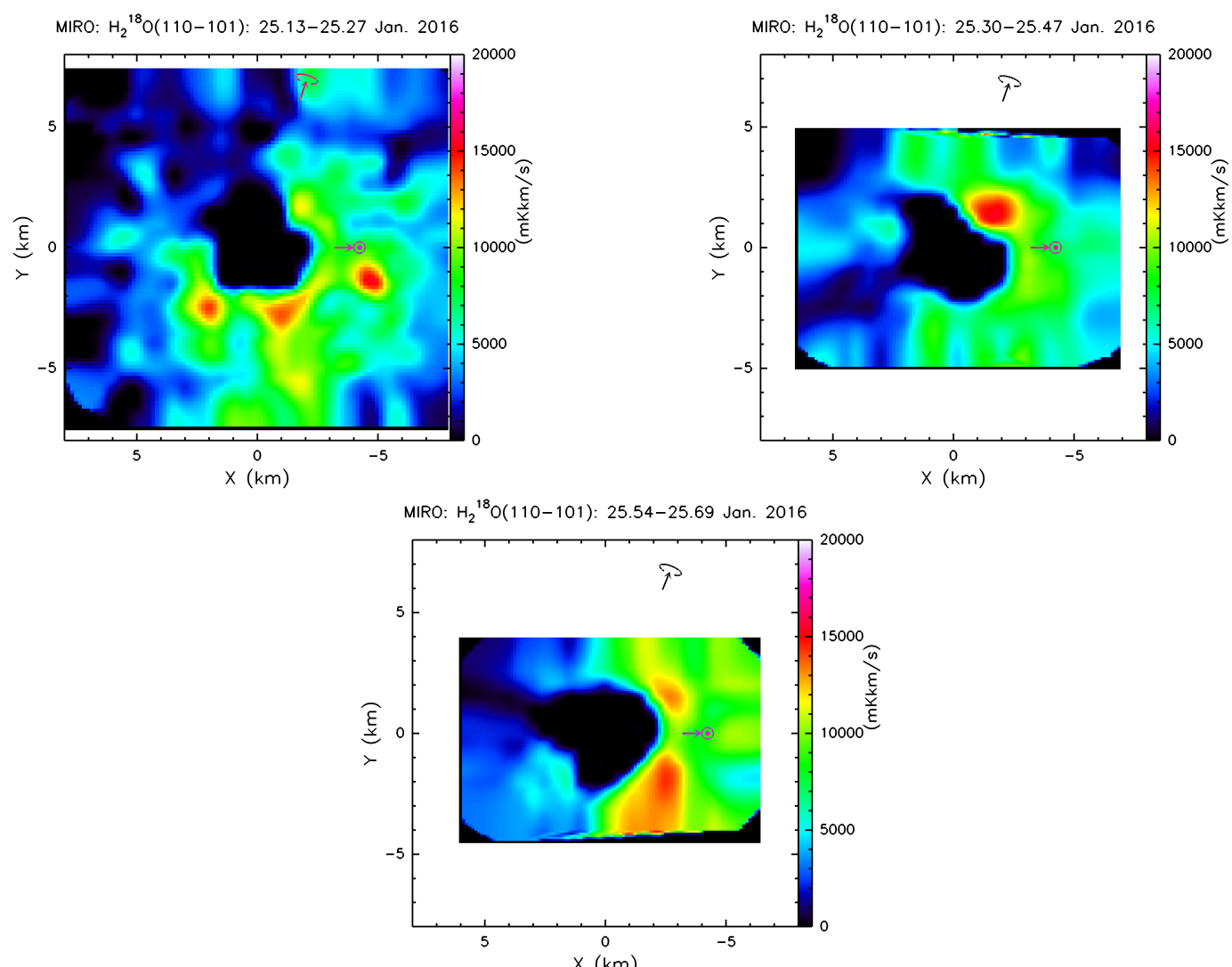

MIRO: $\mathrm{H}_{2}{ }^{18} \mathrm{O}(110-101): 06.27-07.01$ Feb. 2016
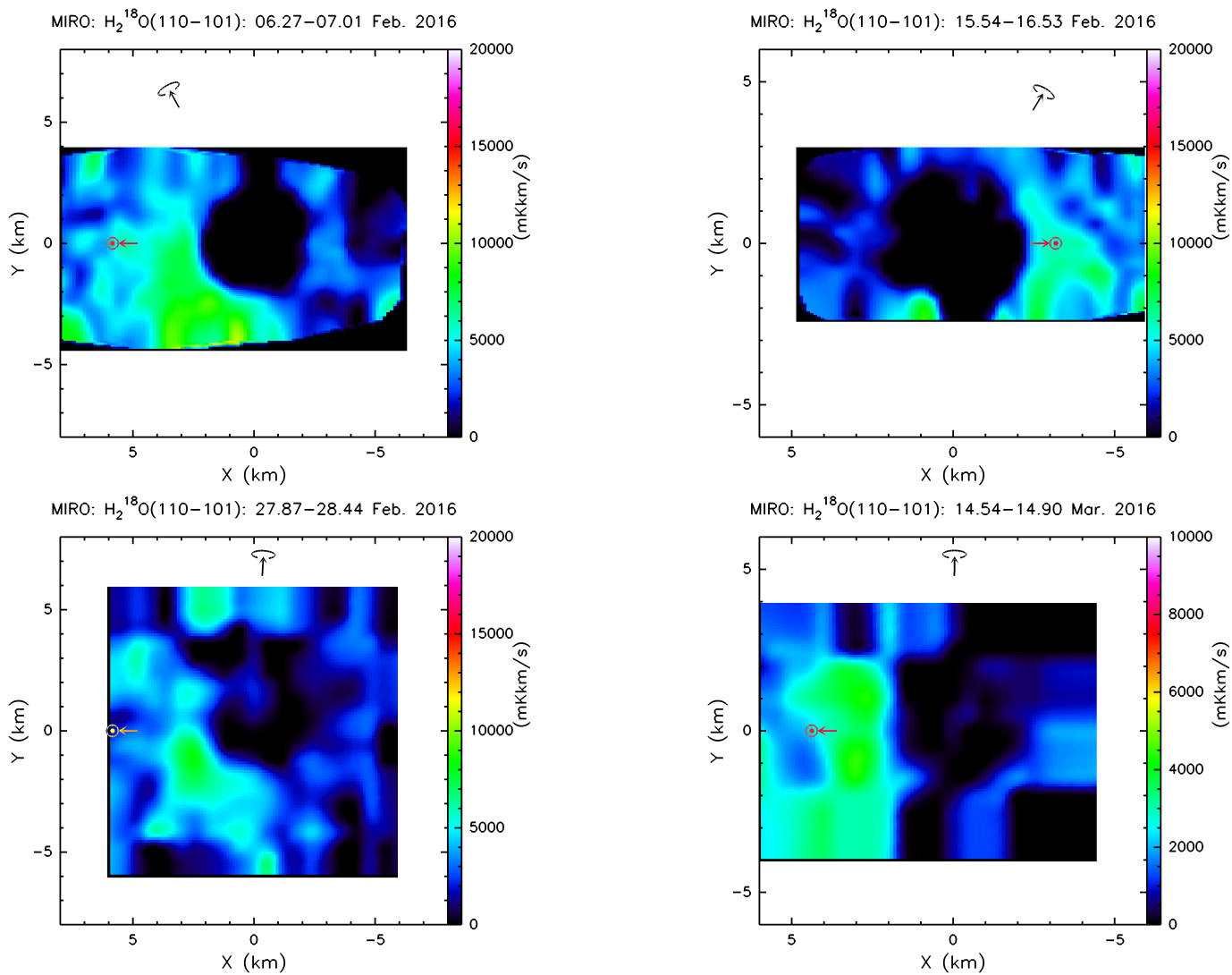

Fig. B.13. Maps of interpolated $\mathrm{H}_{2}^{18} \mathrm{O}$ line intensity on 25 January 2016 and daily averages for 6, 15, and 28 February and 14 March 2016. 
A\&A 630, A19 (2019)
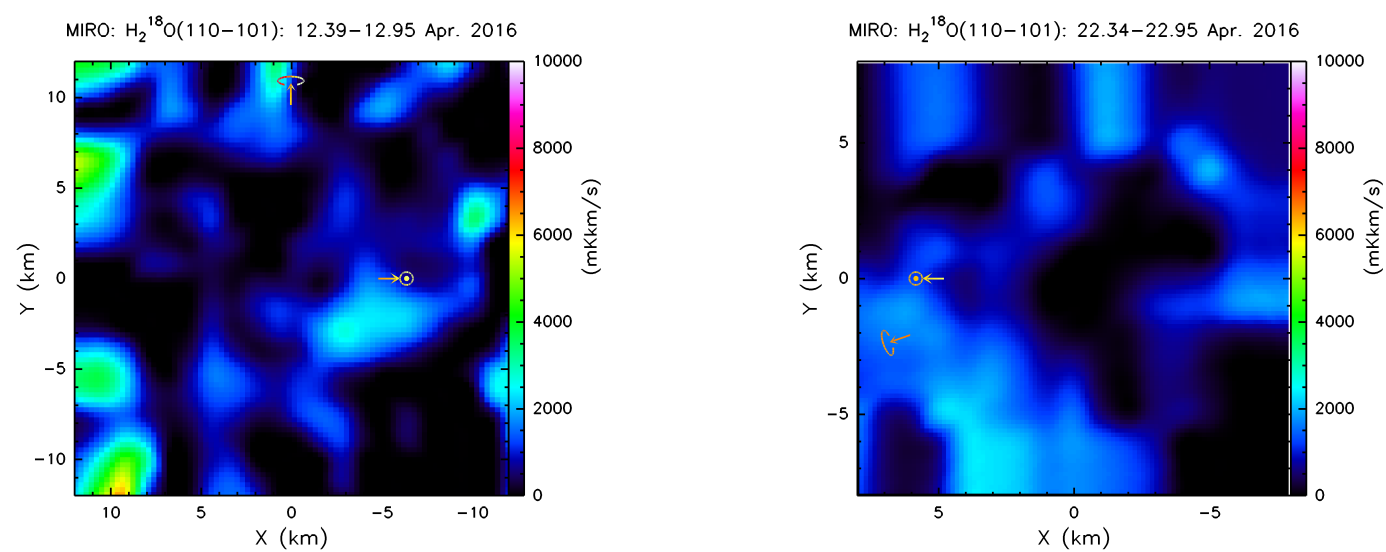

Fig. B.14. Maps (daily average) of interpolated $\mathrm{H}_{2}^{18} \mathrm{O}$ line intensity on 12 and 22 April 2016.

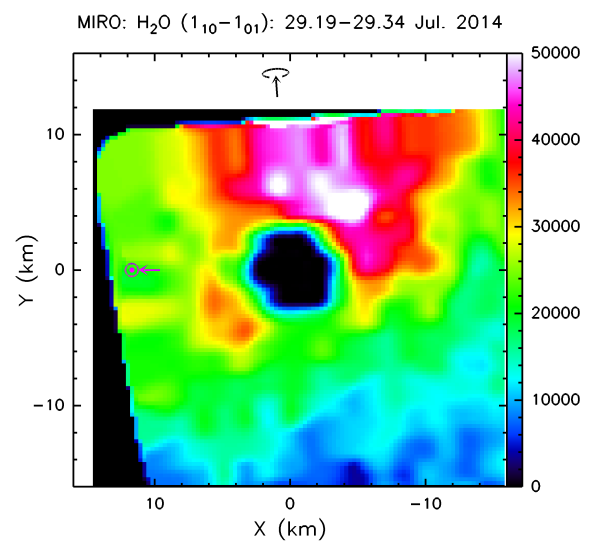

Fig. B.15. Map of interpolated $\mathrm{H}_{2}^{16} \mathrm{O}$ line intensity on 29 July 2014. 


\section{Appendix C: Temperature maps: peak value $T_{\mathrm{mb}}$ of the $\mathrm{H}_{2}^{16} \mathrm{O}$ line}
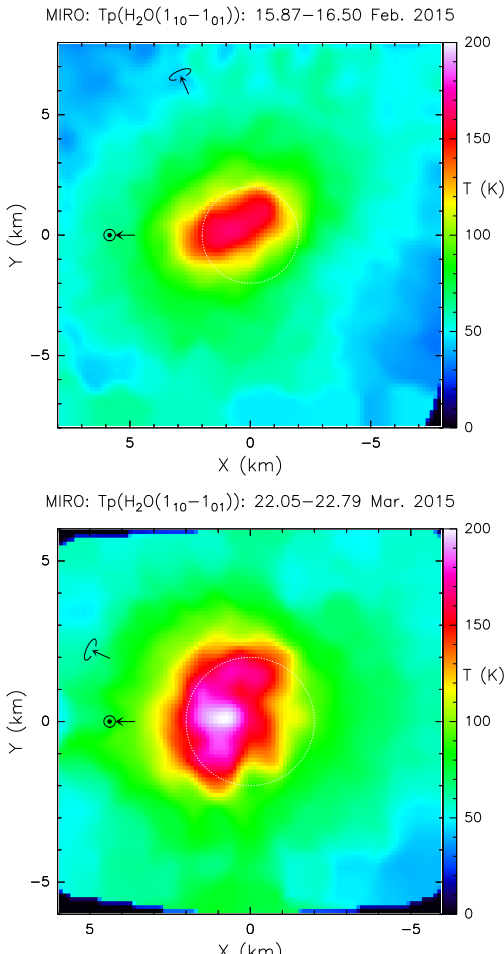

MIRO: $\operatorname{Tp}\left(\mathrm{H}_{2} \mathrm{O}\left(1_{10}-1_{01}\right)\right): 24.45-25.03$ Jun. 2015

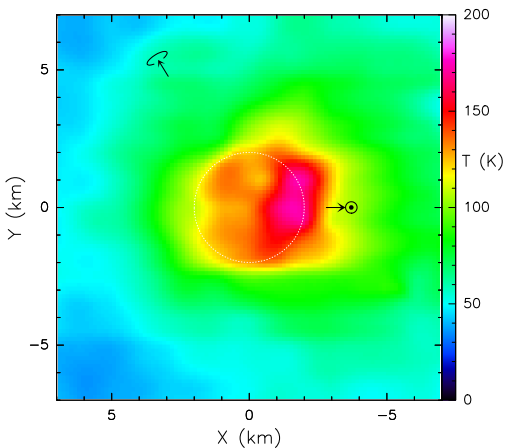

MIRO: $\operatorname{Tp}\left(\mathrm{H}_{2} \mathrm{O}\left(1_{10}-1_{01}\right)\right): 23.31-23.46 \mathrm{Jul} .2015$

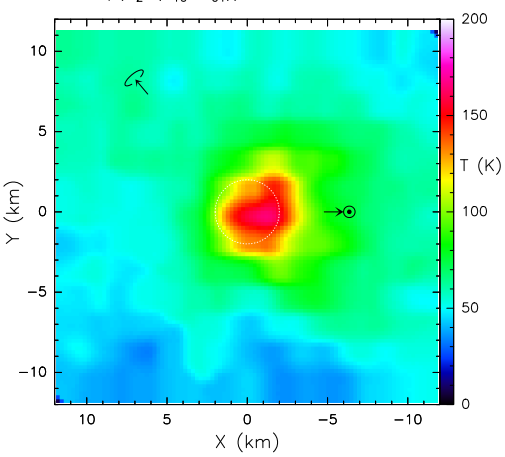

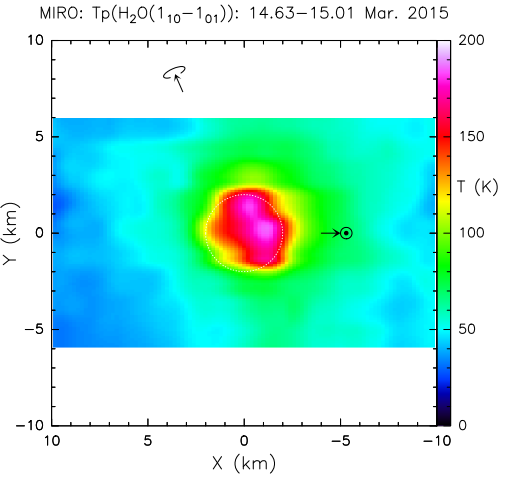

MIRO: $\operatorname{Tp}\left(\mathrm{H}_{2} \mathrm{O}\left(1_{10}-1_{01}\right)\right): 14.05-14.44$ Jun. 2015

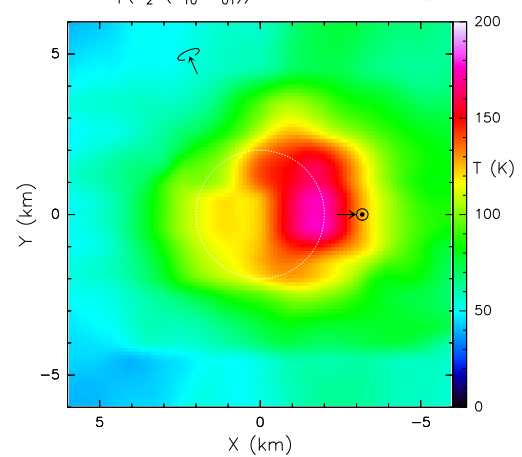

MIRO: $\operatorname{Tp}\left(\mathrm{H}_{2} \mathrm{O}\left(1_{10}-1_{01}\right)\right): 13.12-13.27 \mathrm{Jul} .2015$

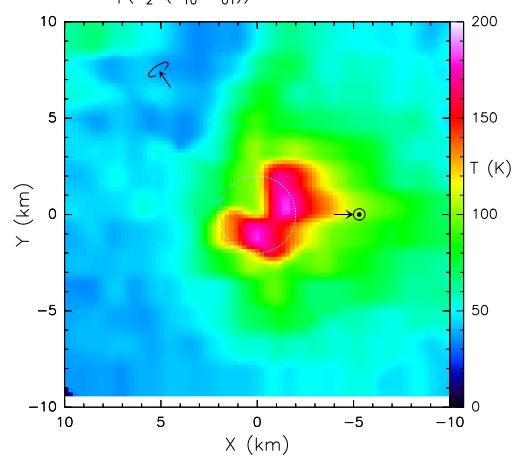

MIRO: $\operatorname{Tp}\left(\mathrm{H}_{2} \mathrm{O}\left(1_{10^{-1}} \mathrm{1}_{01}\right)\right): 30.31-30.88 \mathrm{Jul} .2015$

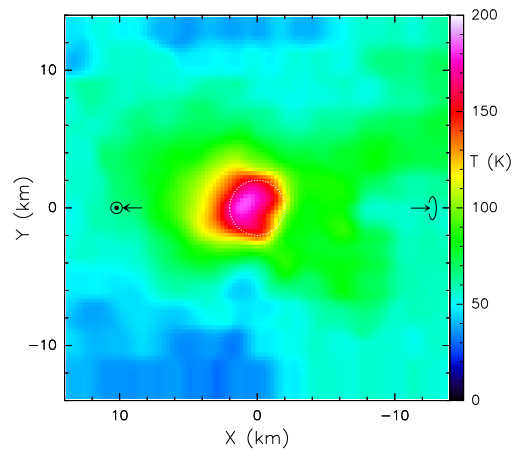

Fig. C.1. Sample of the interpolated maps of the peak intensity of the $\mathrm{H}_{2}^{16} \mathrm{O}$ line, which is a proxy (when saturated) for the gas temperature (-10 K because of the difference between the blackbody temperature and the Rayleigh-Jeans approximation used for the $T_{\mathrm{mb}}$ scale). The 20 June $2016 \mathrm{map}$ shown here was not used because the $\mathrm{H}_{2}^{16} \mathrm{O}$ line is no longer saturated at the local gas temperature: $T p\left(\mathrm{H}_{2}^{16} \mathrm{O}\right)$ decreases out to $\approx 0 \mathrm{~K}$ because of the low column density. 
A\&A 630, A19 (2019)
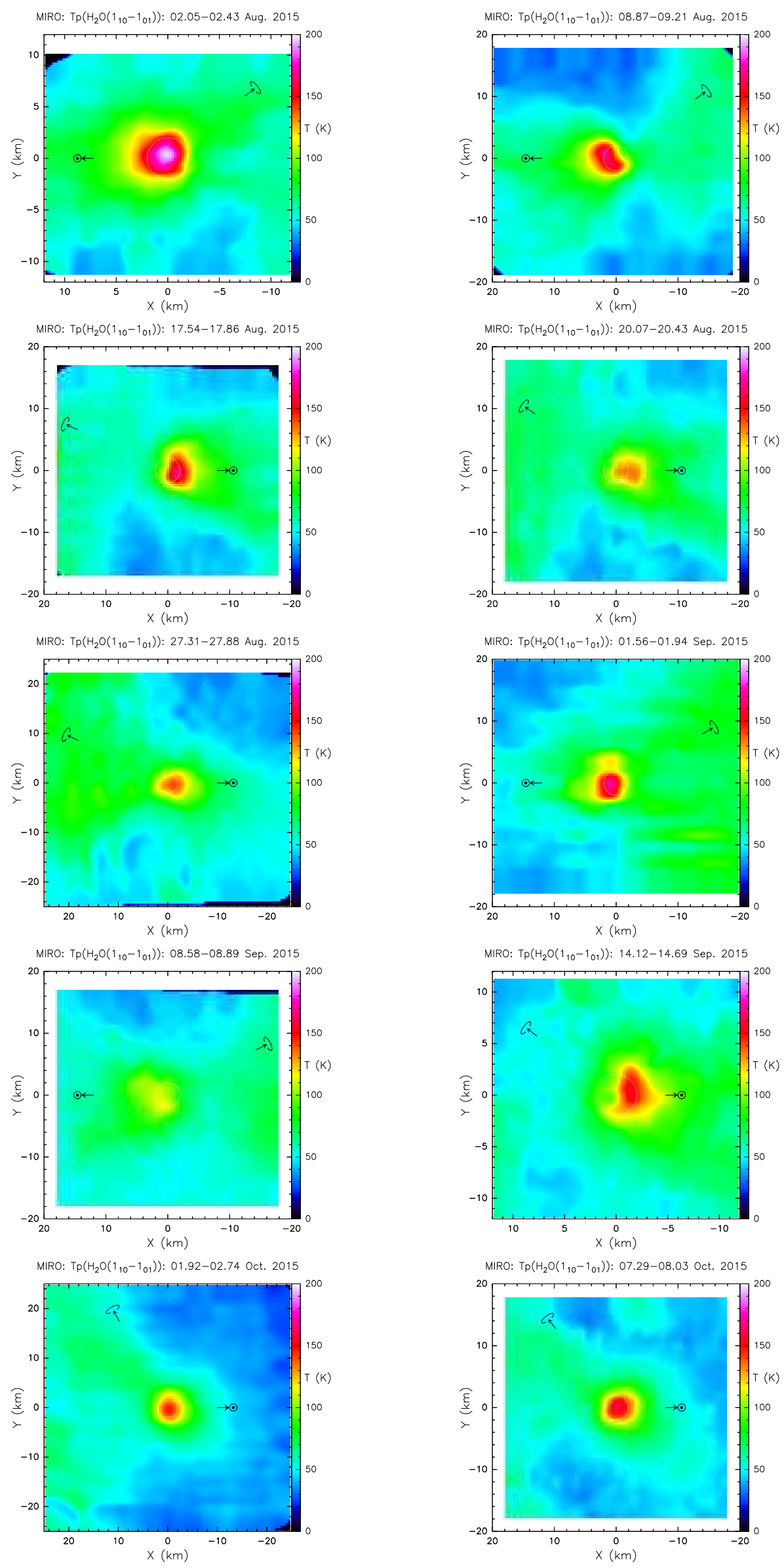

Fig. C.1. continued. 
N. Biver et al.: Monitoring of comet 67P with Rosetta/MIRO

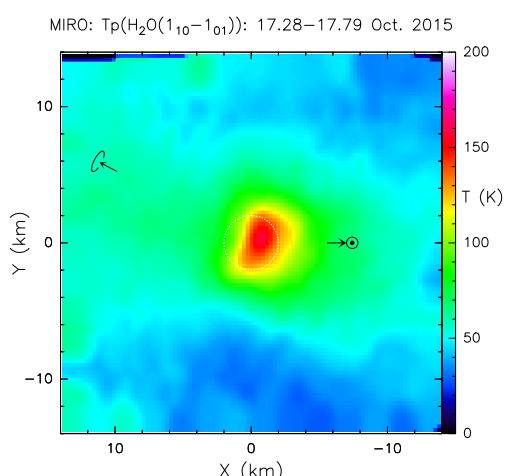

MIRO: $\operatorname{Tp}\left(\mathrm{H}_{2} \mathrm{O}\left(1_{10}-1_{01}\right)\right)$ : 05.07-05.88 Nov. 2015

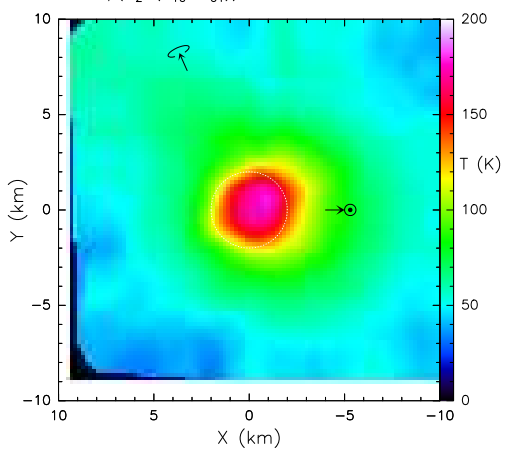

MIRO: $\operatorname{Tp}\left(\mathrm{H}_{2} \mathrm{O}\left(1_{10}-1_{01}\right)\right): 15.29-15.85$ Nov. 2015
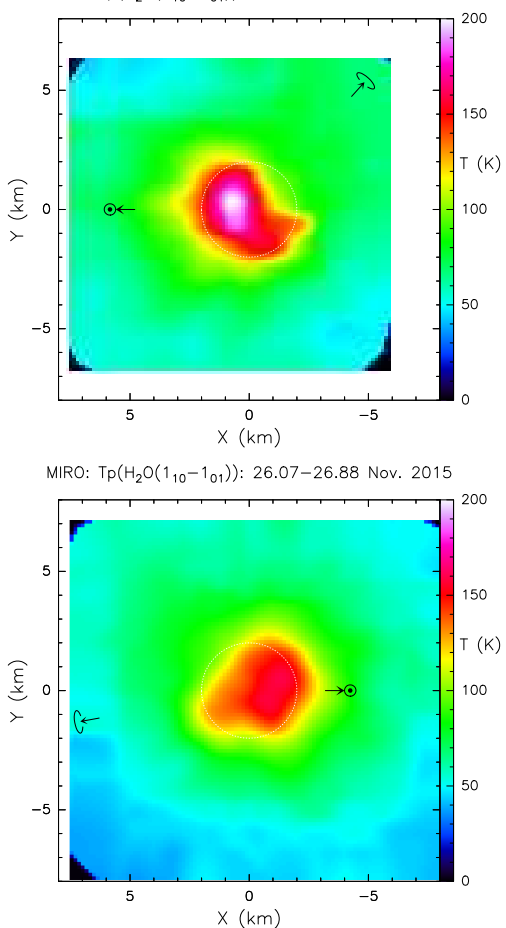

MIRO: $\operatorname{Tp}\left(\mathrm{H}_{2} \mathrm{O}\left(1_{10}-1_{01}\right)\right): 22.61-22.94$ Dec. 2015

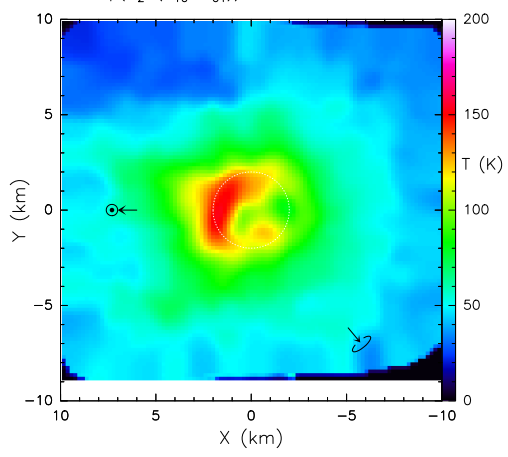

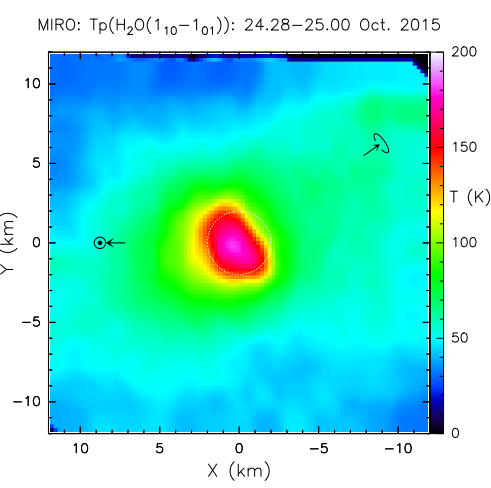

MIRO: $\operatorname{Tp}\left(\mathrm{H}_{2} \mathrm{O}\left(1_{10}-1_{01}\right)\right): 11.29-11.82$ Nov. 2015

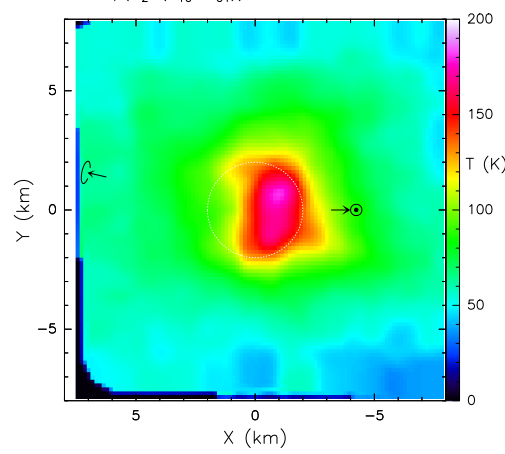

MIRO: $\operatorname{Tp}\left(\mathrm{H}_{2} \mathrm{O}\left(1_{10}-1_{01}\right)\right): 21.87-22.43$ Nov. 2015
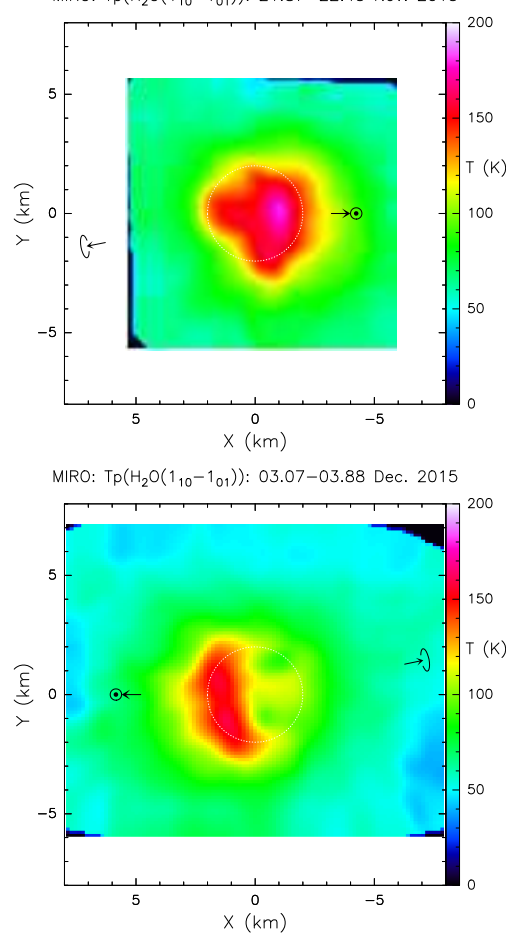

MIRO: $\operatorname{Tp}\left(\mathrm{H}_{2} \mathrm{O}\left(1_{10}-1_{01}\right)\right): 23.30-23.81$ Dec. 2015

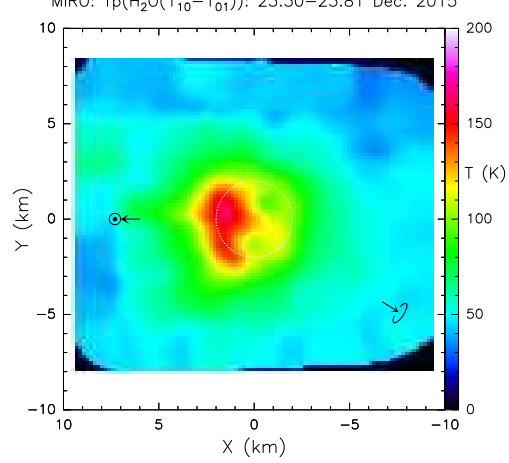

Fig. C.1. continued. 
A\&A 630, A19 (2019)
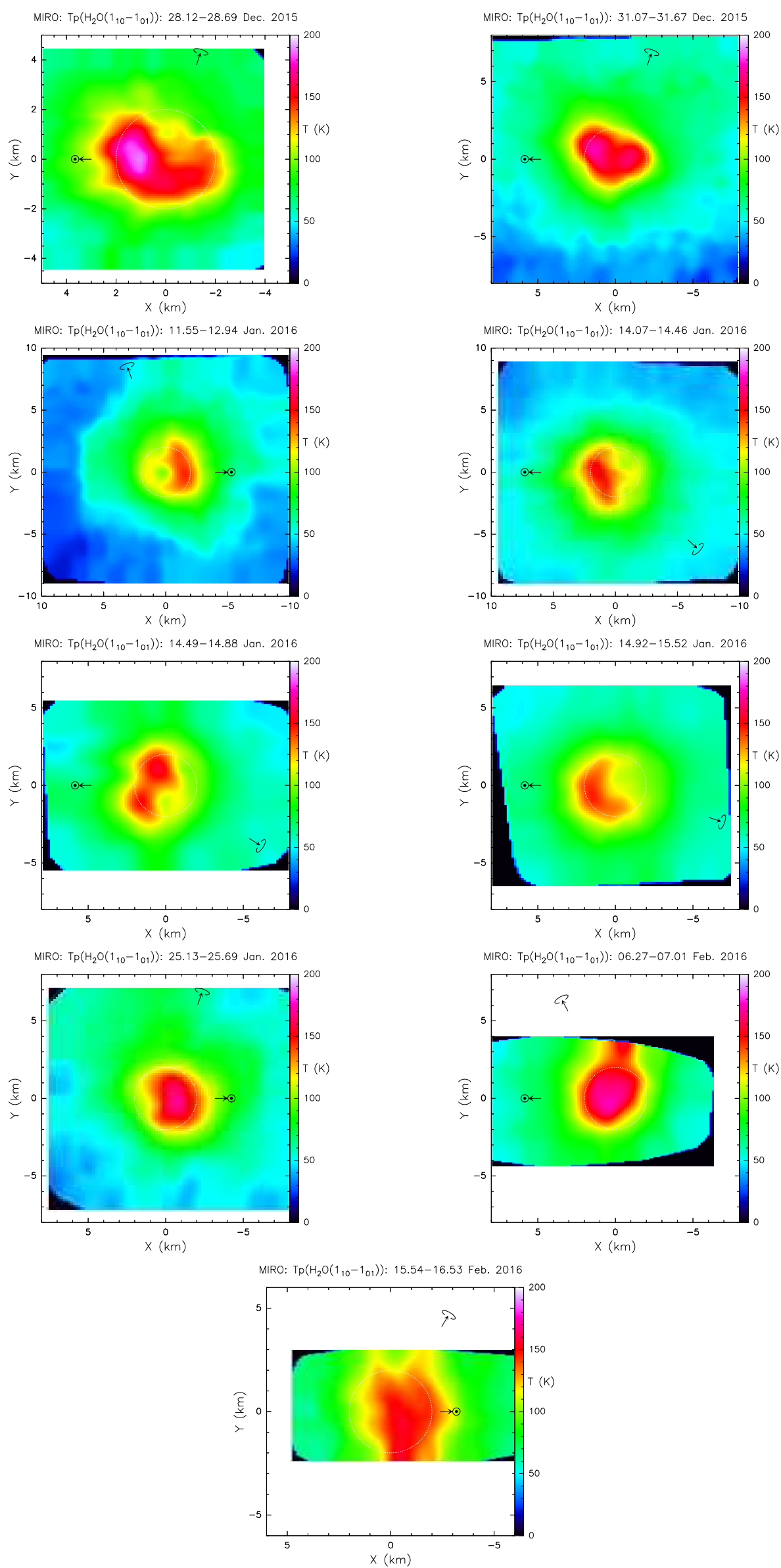

Fig. C.1. continued.

A19, page 52 of 58 
N. Biver et al.: Monitoring of comet 67P with Rosetta/MIRO
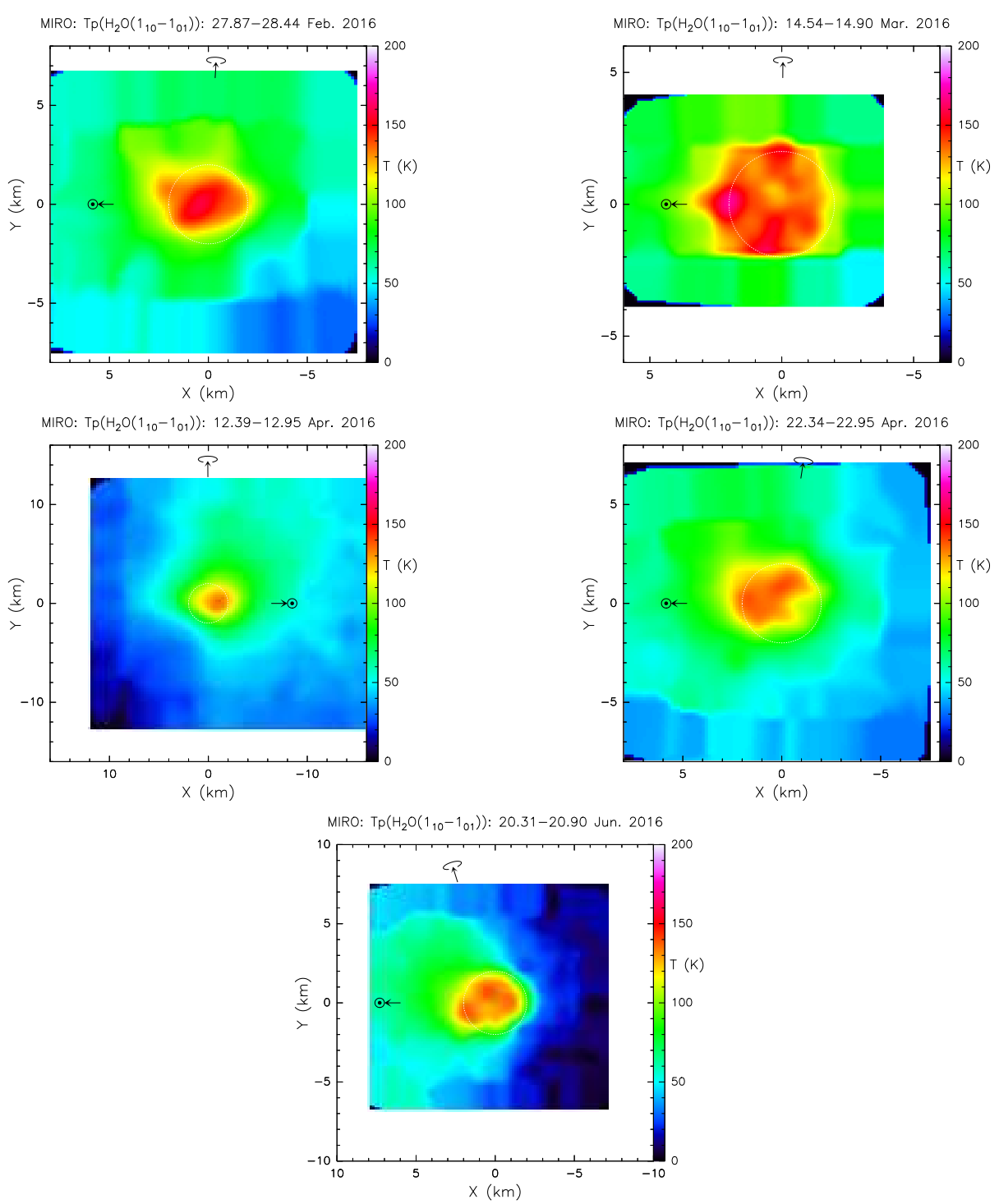

Fig. C.1. continued. 


\section{Appendix D: Column densities measured across the jet or night side}

In this section we show the evolution of the derived column densities across the night- or day-side of the coma along the projected axis of the jet. The shaded region corresponds to pointings with the nucleus in the background. Column densities with the nucleus in the background are estimated for an altitude above $2 \mathrm{~km}$ from the center of the nucleus. They are very uncertain because the density cannot be precisely evaluated for the warm gas close to the surface.
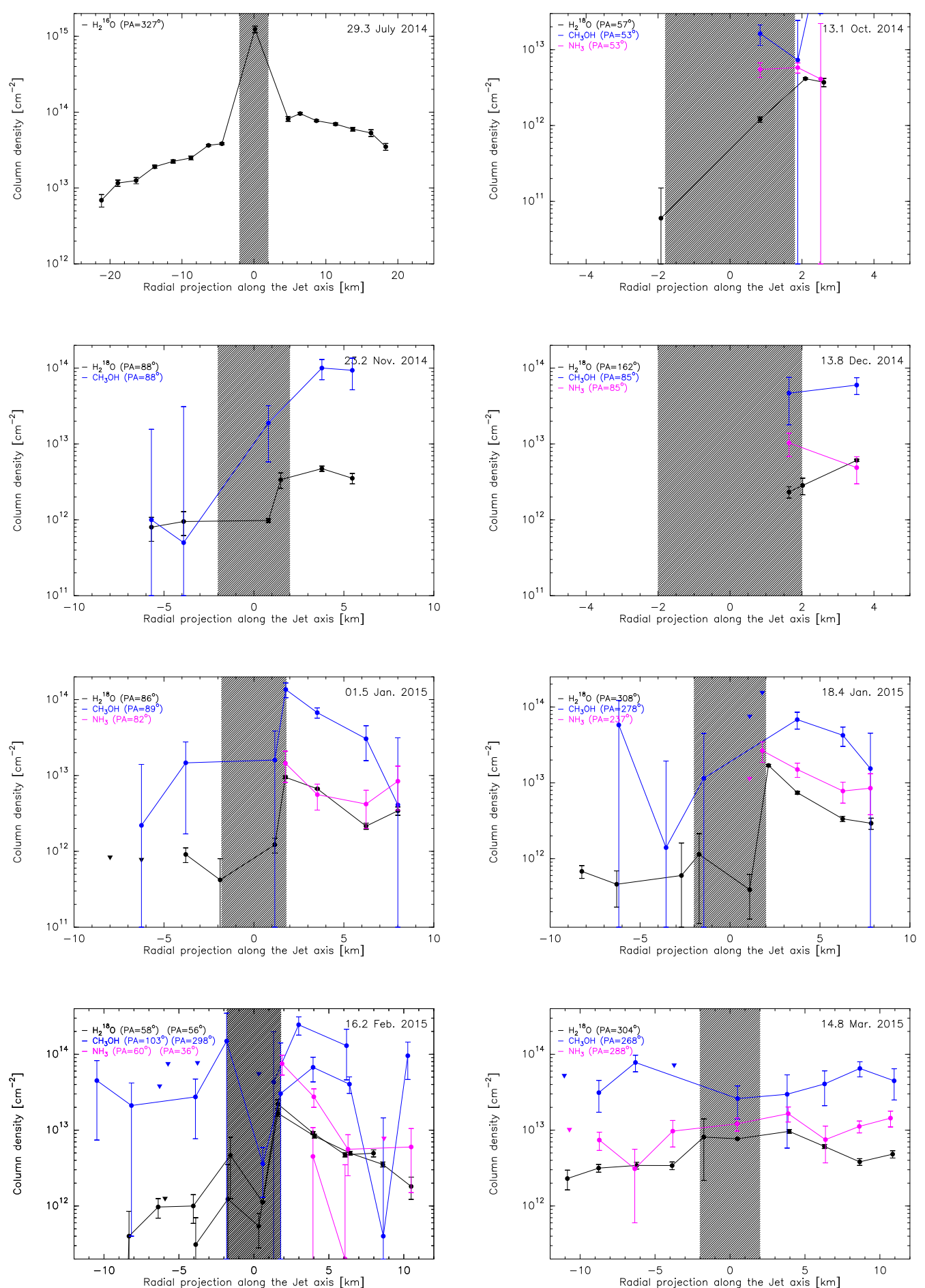

Fig. D.1. $\mathrm{H}_{2}^{16} \mathrm{O}$ or $\mathrm{H}_{2}^{18} \mathrm{O}, \mathrm{CH}_{3} \mathrm{OH}, \mathrm{NH}_{3}$, and $\mathrm{CO}$ column densities in the coma derived by the MIRO instrument from the maps (date indicated in each plot). Each measurement is derived from the average line intensity from points within $2.5 \mathrm{~km}$ projected distance from the nucleus and $60^{\circ}$ in position angle (PA) over the sky along the comet jet axis. The mean jet PA is provided in the upper left corner for each molecule. The horizontal axis is the projected distance from the nucleus on the jet (Sun) side for positive values. The vertical scale is the derived column density in $\mathrm{cm}^{-2}$. The hatched region corresponds to pointing against the nucleus (line in absorption). 
N. Biver et al.: Monitoring of comet 67P with Rosetta/MIRO
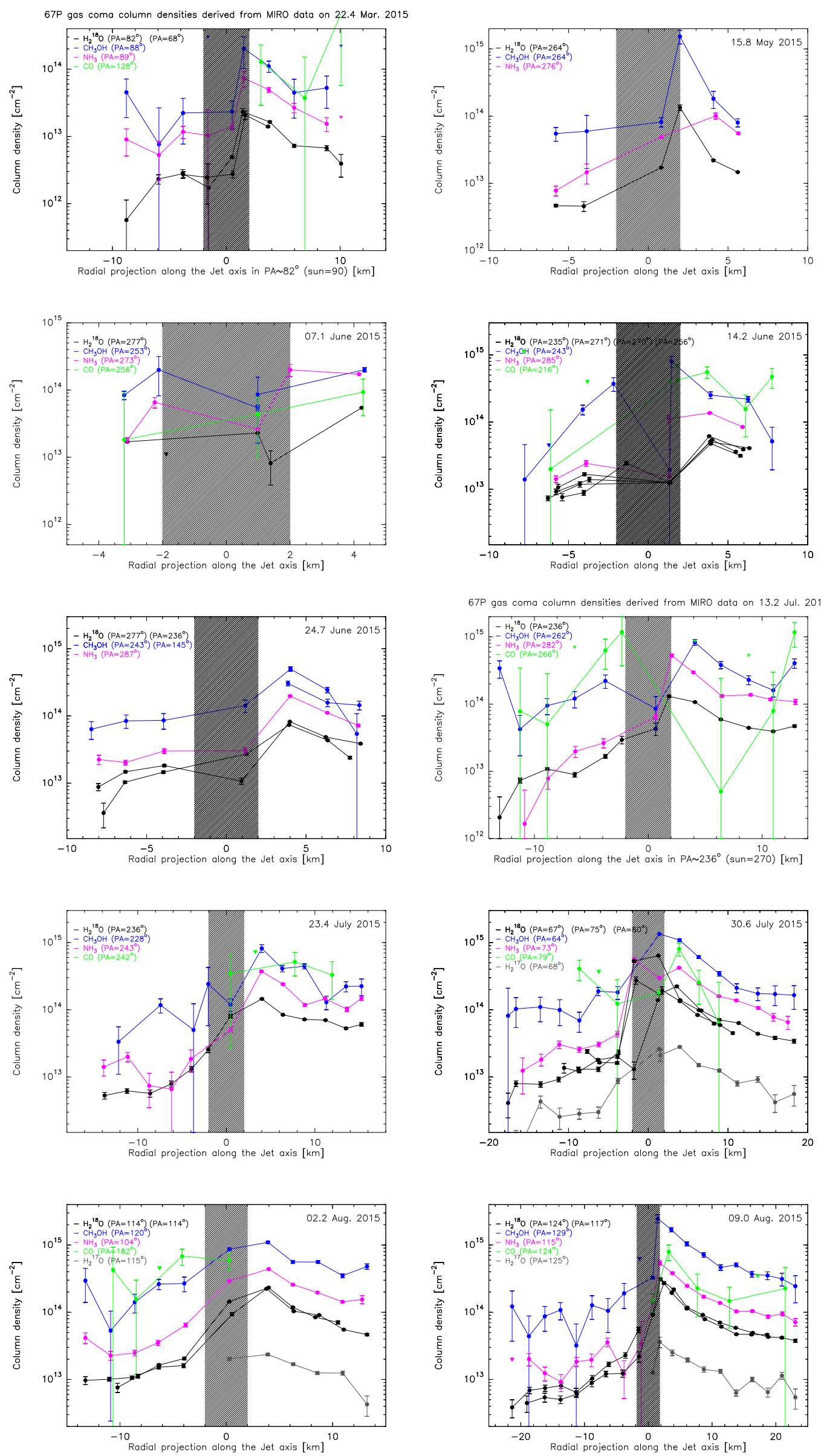

Fig. D.1. continued. 
A\&A 630, A19 (2019)
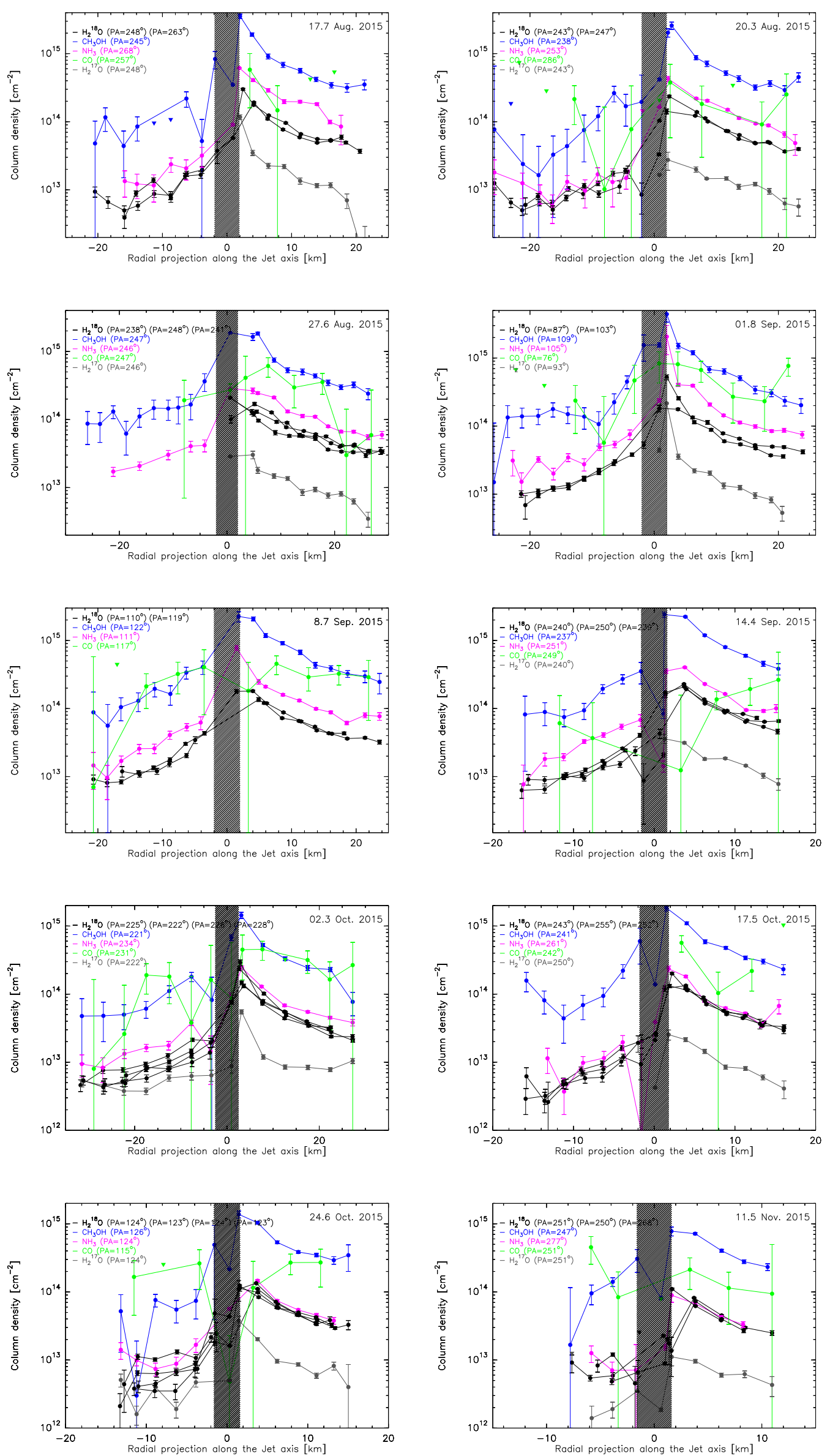

Fig. D.1. continued.

A19, page 56 of 58 
N. Biver et al.: Monitoring of comet 67P with Rosetta/MIRO
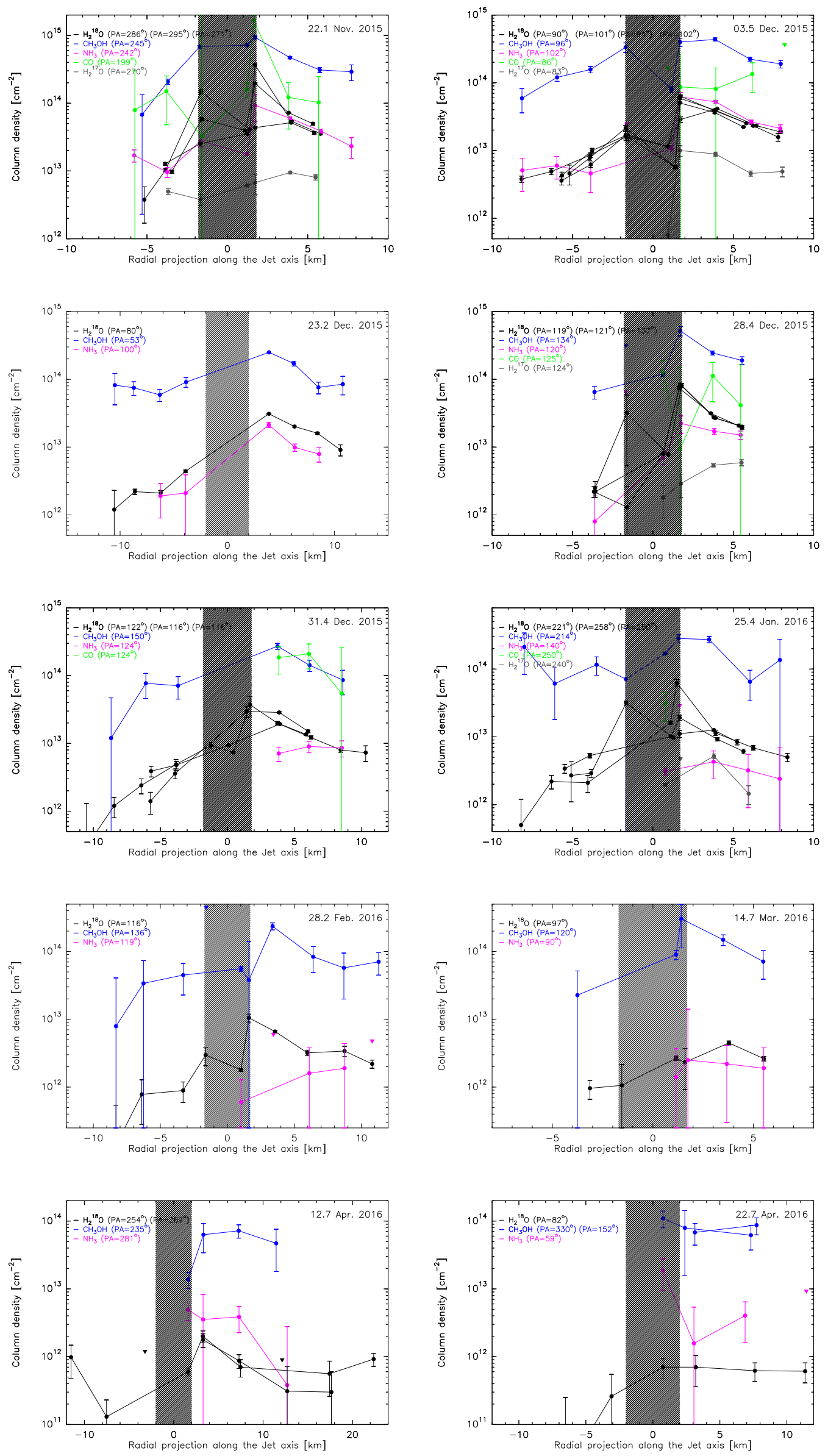

Fig. D.1. continued. 


\section{Appendix E: Examples of nadir pointing spectra}
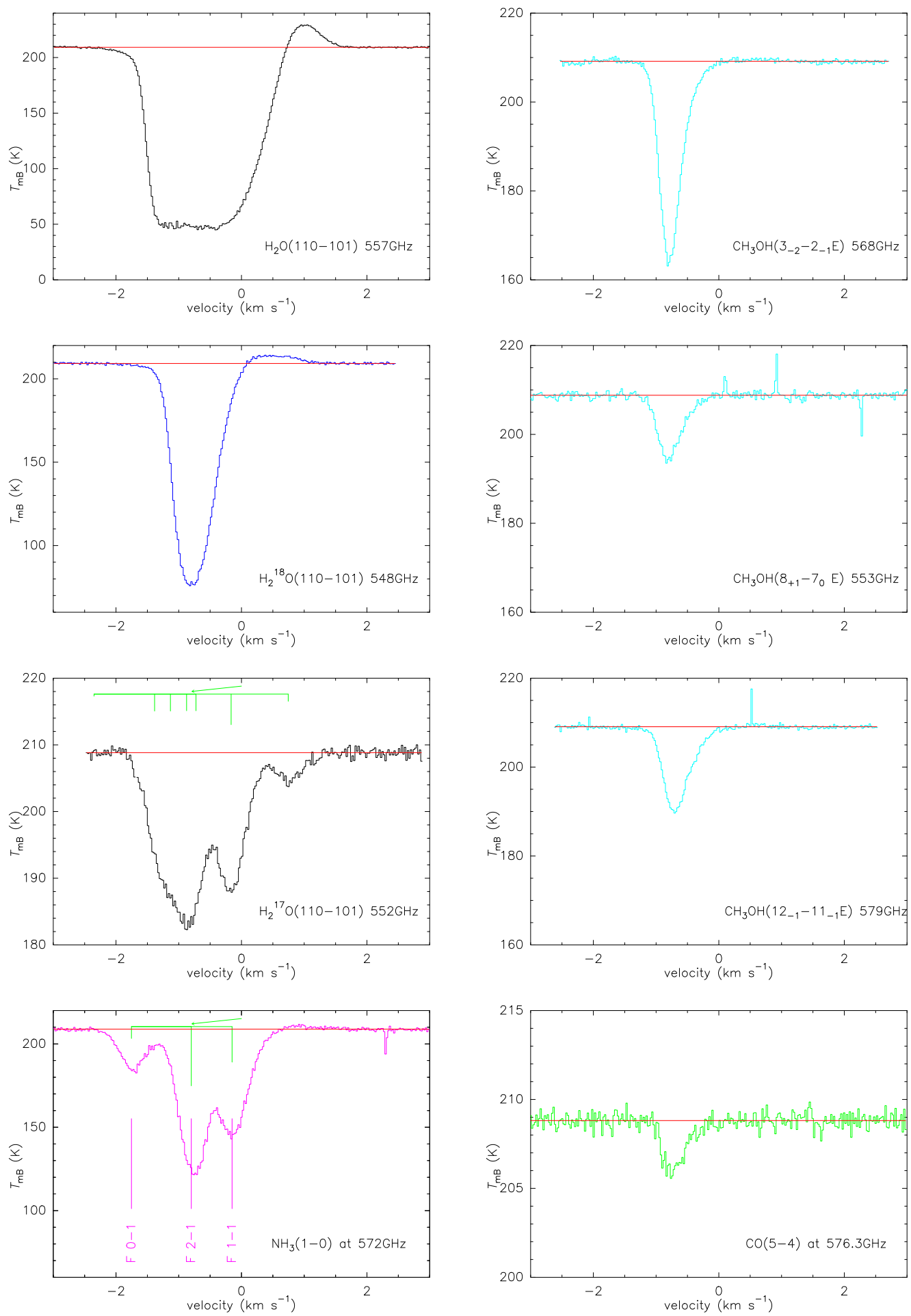

Fig. E.1. Nadir pointing spectra obtained between 30.51 and 31.00 August 2015. The vertical scale is main beam brightness temperature in K. The horizontal scale is Doppler velocity relative to the nucleus. Lines are seen in absorption against the nucleus background temperature at $209 \mathrm{~K}$ and are blueshifted by $-0.8 \mathrm{~km} \mathrm{~s}^{-1}$ because most of the gas flows toward MIRO/Rosetta. The position and expected relative intensities of the hyperfine components of the $\mathrm{H}_{2}^{17} \mathrm{O}$ and $\mathrm{NH}_{3}$ lines are shown. They are displaced by $-0.8 \mathrm{~km} \mathrm{~s}^{-1}$, which is the mean gas bulk velocity as seen in other lines. Narrow spikes, which are instrument artifacts, appear regularly in the spectra of ammonia and two of the methanol lines. 